\title{
Simulation based modeling of the elastic properties of structural wood based composite lumber
}

\author{
Laszlo Bejo \\ West Virginia University
}

Follow this and additional works at: https://researchrepository.wvu.edu/etd

\section{Recommended Citation}

Bejo, Laszlo, "Simulation based modeling of the elastic properties of structural wood based composite lumber" (2001). Graduate Theses, Dissertations, and Problem Reports. 1385.

https://researchrepository.wvu.edu/etd/1385

This Dissertation is protected by copyright and/or related rights. It has been brought to you by the The Research Repository @ WVU with permission from the rights-holder(s). You are free to use this Dissertation in any way that is permitted by the copyright and related rights legislation that applies to your use. For other uses you must obtain permission from the rights-holder(s) directly, unless additional rights are indicated by a Creative Commons license in the record and/ or on the work itself. This Dissertation has been accepted for inclusion in WVU Graduate Theses, Dissertations, and Problem Reports collection by an authorized administrator of The Research Repository @ WVU.

For more information, please contact researchrepository@mail.wvu.edu. 


\title{
Simulation Based Modeling of the Elastic Properties of Structural Wood Based Composite Lumber
}

\author{
Laszlo Bejo
}

Dissertation submitted to the College of Agriculture, Forestry and Consumer Sciences at West Virginia University in partial fulfillment of the requirements for the degree of

\author{
Doctor of Philosophy \\ in \\ Forest Resource Science
}

\author{
Elemer M. Lang, Ph.D., Chair \\ James P. Armstrong, Ph.D. \\ R. Bruce Anderson, Ph.D. \\ Julio F. Davalos, Ph.D. \\ Benjamin Dawson-Andoh, Ph.D. \\ Division of Forestry
}
Morgantown, West Virginia 2001

Keywords: Orthotropy, Elastic Properties, Simulation, Composite Lumber 


\section{ABSTRACT \\ Simulation Based Modeling of the Elastic Properties of Structural Wood Based Composite Lumber}

\section{Laszlo Bejo}

The importance of wood-based composite lumber is increasing in the US market for construction materials. Manufacturers of such composites strive to make their products more competitive by increasing their value. This dissertation describes the development of simulation models that can aid these efforts by estimating the elastic characteristics of composite lumber products. The study included the assessment of the orthotropic mechanical properties of the raw material, the effect of densification it experiences during the hot-pressing procedure, and the geometric structure of the composites. Using the results of these investigations, computer models were created based on principles of deterministic and stochastic simulations. Generated elastic parameters were validated against experimentally measured MOE values. Reasonably good agreement between the simulated and actual elastic constants confirmed the usefulness of the developed models. The simulations can be used to explore the characteristics of composite beams with innovative designs or containing new raw materials before they enter the prototype phase of their development. 


\section{ACKNOWLEDGEMENTS}

The author would like to express sincere appreciation to Dr. Elemer M. Lang, who initiated, helped and motivated his efforts throughout this project. Without his valuable advice, as well as his almost fatherly involvement, this work would never have been completed. Appreciation is also extended to Dr. R. Bruce Anderson, Dr. James P. Armstrong, Dr. Benjamin Dawson-Andoh and Dr. Julio P. Davalos, as well as the faculty and staff of the Wood Science Extenison and the Appalachian Hardwood Center. Special thanks to Dr. William Thayne who provided invaluable help in solving many of the statistical problems that emerged during the analytical work, as well as to Dr. Ferenc Divos, Dr. Jozsef Szalai of the West Hungarian University, whose expertise was indispensable. Technical assistance and raw material donation by Trus Joist, A Weyerhaeuser Business, Buckhannon, WV is gratefully acknowledged.

I would also like to thank all my friends and my brothers and sisters in Jesus Christ for all the encouragement their words, prayers and help meant in the last three years. I owe tremendous appreciation to my whole family, especially to my parents for their generous support in every way. Finally and most importantly, I am deeply indebted to my wife, Gyongyi Bejo, whose patience, support, love, encouragement and undeserved admiration kept me sane and focused in this very challenging period of my life. 


\section{TABLE OF CONTENTS}

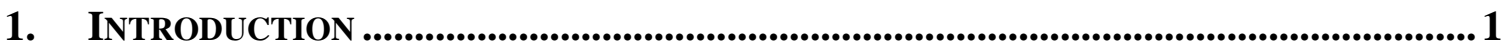

$1.1 \quad$ Laminated Veneer Lumber (LVL) ............................................................................ 2

$1.2 \quad$ Parallel Strand Lumber (PSL) ....................................................................... 4

1.3 Simulation modeling .....................................................................................5

2. OBJECTIVES AND DISSERTATION STRUCTURE _....................................................

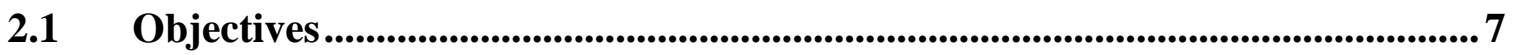

$2.2 \quad$ Structure of the dissertation......................................................................................... 7

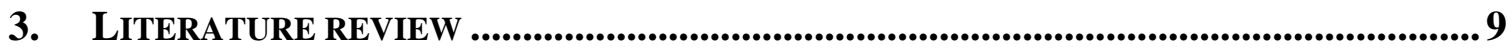

3.1 Orthotropic strength and elasticity of solid wood ..................................................9

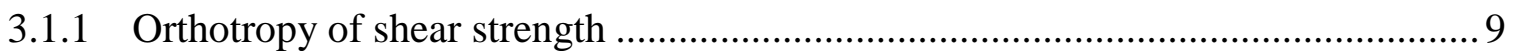

3.1.2 Orthotropy of compression strength and elasticity …….................................. 12

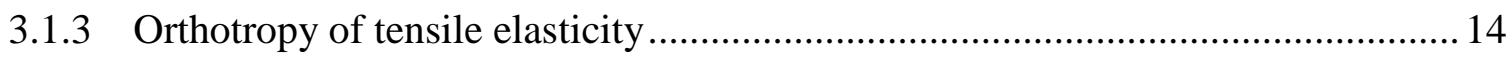

3.2 Modification of constituents' properties during manufacture........................... 17

3.3 Wood composite modeling............................................................................19

4 THEORETICAL BACKGROUND .....................................................................................24

4.1 Orthotropic strength and elasticity …...................................................................2 24

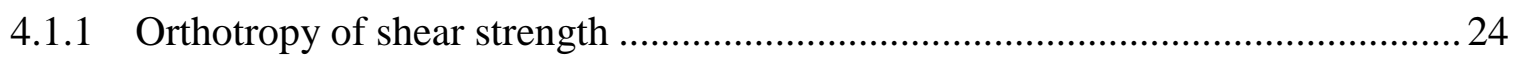

4.1.2 Orthotropy of compression strength and elasticity ………..................................... 32

4.1.2.1 Shear stresses in compression specimens .............................................................. 32

4.1.2.2 Models describing the orthotropic compression properties ................................. 34

4.1.3 Orthotropy of the dynamic elastic parameters ................................................... 37

4.2 Prediction of MOE by stress-wave propagation.....................................................39

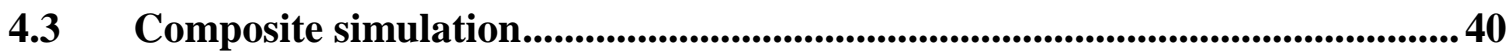

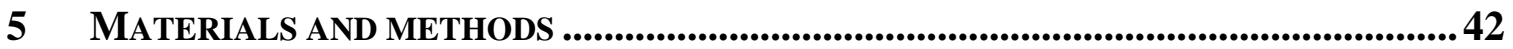

$5.1 \quad$ Orthotropic strength and elasticity ……...............................................................42

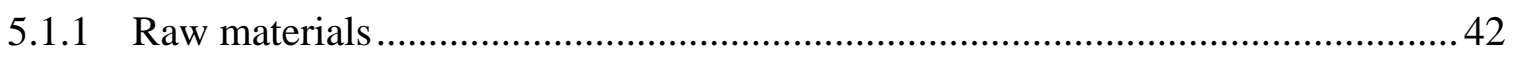

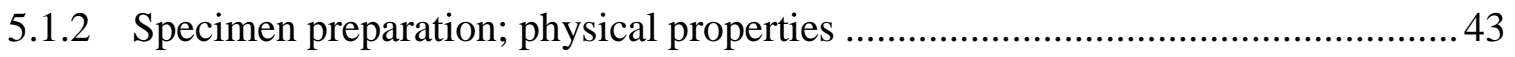

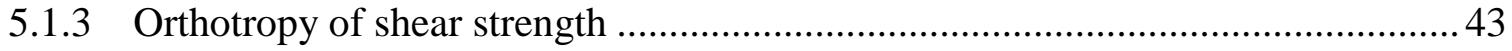

5.1.4 Orthotropy of compression strength and elasticity ……..................................... 47

5.1.5 Orthotropy of tensile elasticity ........................................................................ 49 


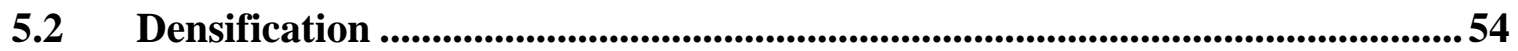

5.3 Mechanical properties of the composites ..........................................................56

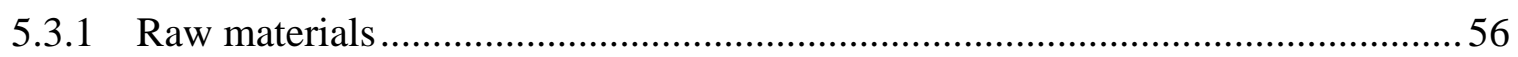

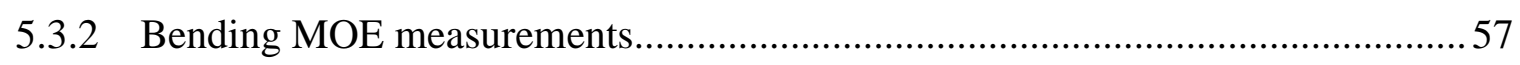

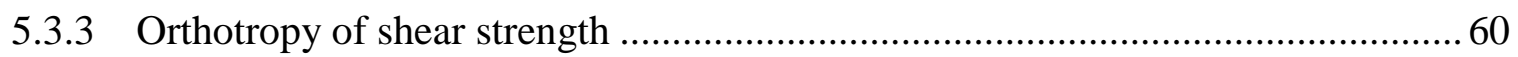

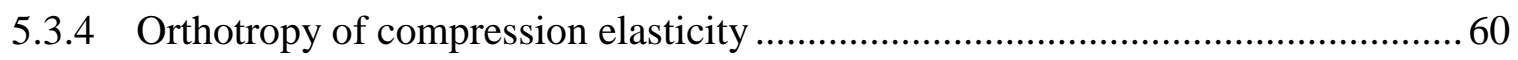

5.4 Composite geometry ..........................................................................................6 61

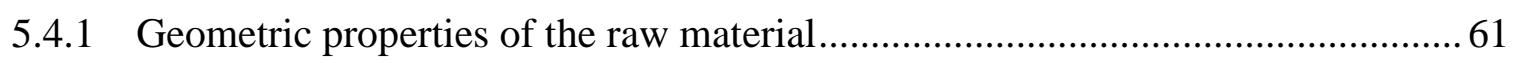

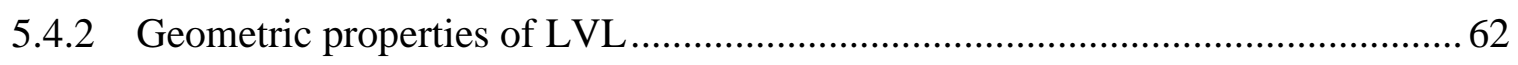

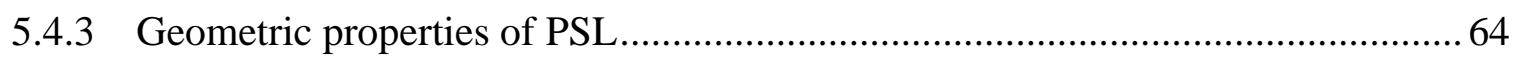

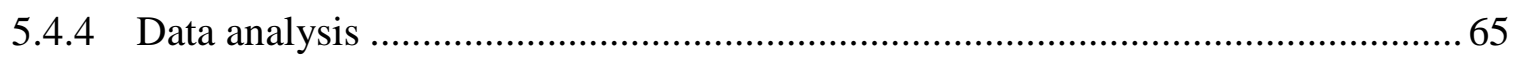

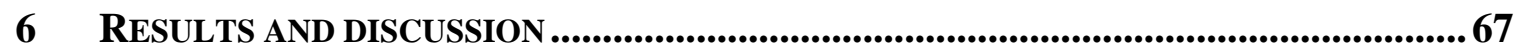

6.1 Orthotropy of the raw material ..............................................................................6 67

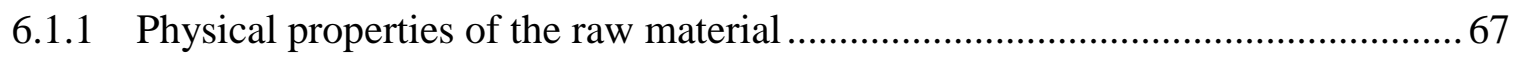

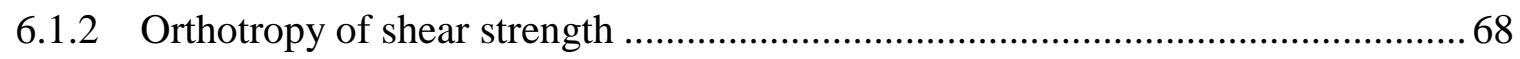

6.1.3 Orthotropy of compression strength and elasticity …………............................. 76

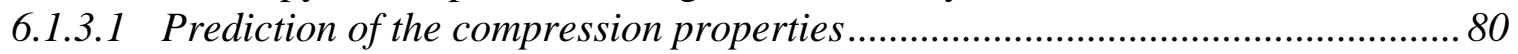

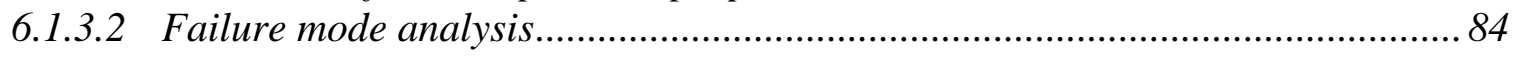

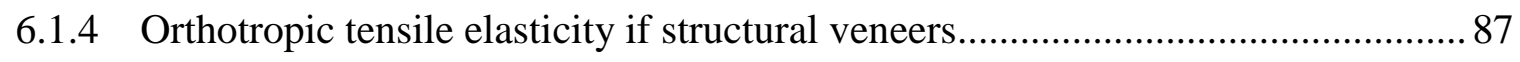

6.2 The effect of densification on the MOE of veneer ................................................96

6.2.1 The effect of densification on the dynamic MOE ..............................................97

6.2.2 Validation by static tensile MOE measurements ............................................... 100

6.3 Mechanical properties of the composites ........................................................ 102

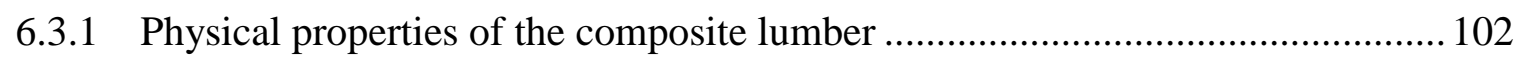

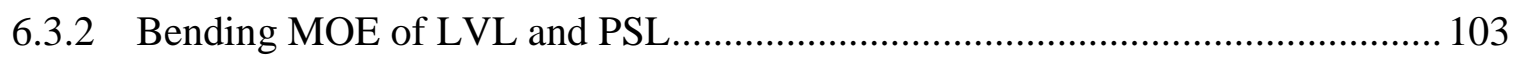

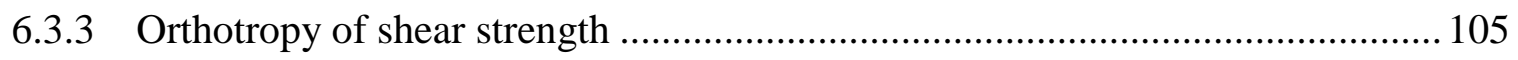

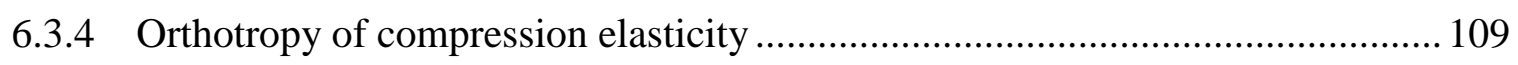

6.4 Composite geometry ....................................................................................................... 111

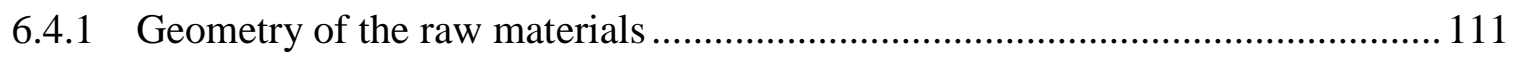

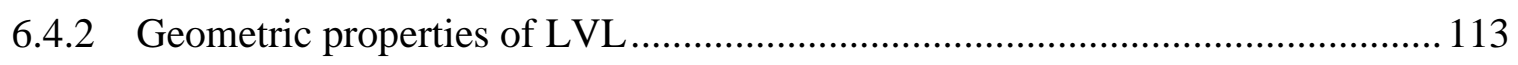

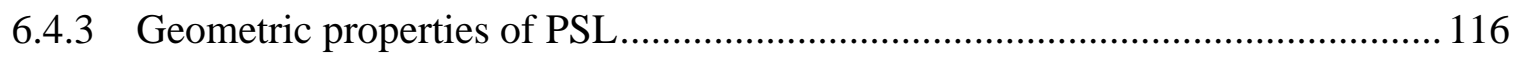

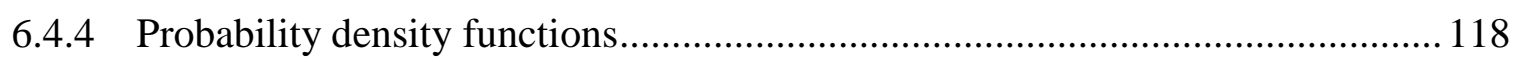


7 MODEL DEVELOPMENT AND VALIDATION ................................................................. 119

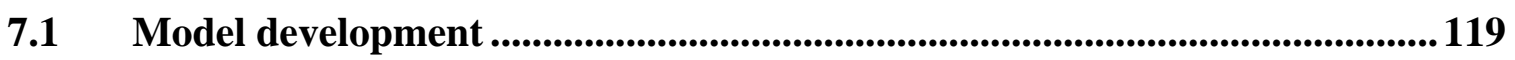

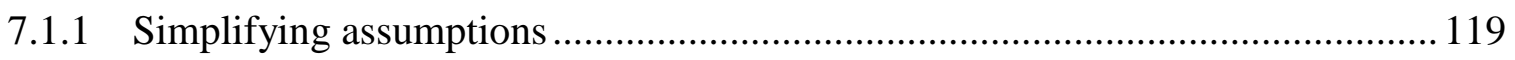

7.1.2 Simulation of the constituents' geometric parameters ....................................... 120

7.1.3 Simulation of the composites' geometric and physical properties....................... 123

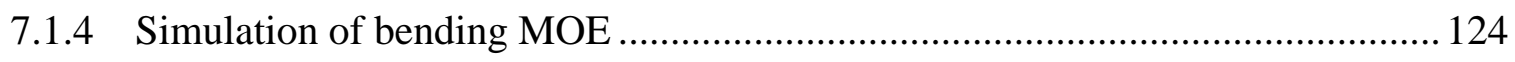

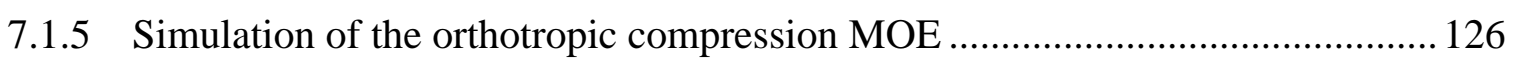

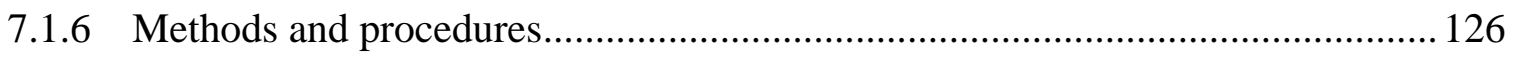

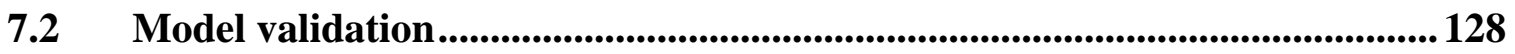

7.2.1 Geometric and physical properties of LVL and PSL ...................................... 129

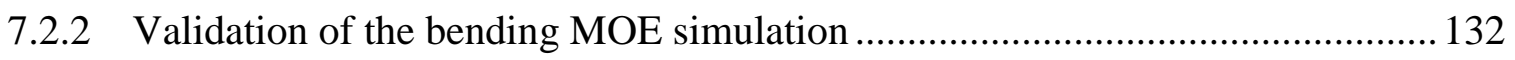

7.2.3 Validation of the orthotropic compression MOE simulation ............................. 135

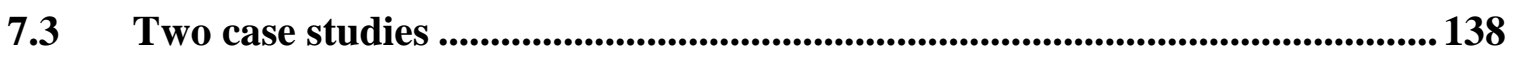

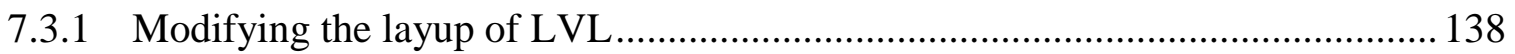

7.3.2 Decreasing the variation of load and strand orientation in PSL......................... 141

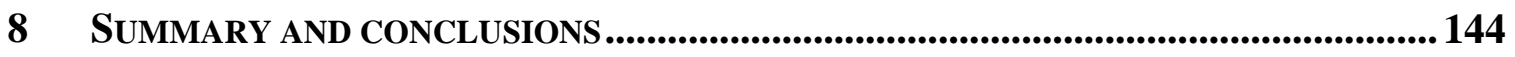

9 RECOMMENDATIONS FOR FURTHER RESEARCH ...................................................... 146

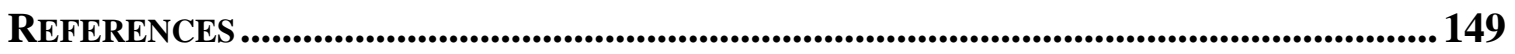

APPENDIX A - DERIVATION OF THE ORTHOTROPIC TENSOR MODEL............................... 156

APPENDIX B - DETAILED RESULTS OF THE STATISTICAL PROCEDURES........................... 160

APPENDIX C - SOME GEOMETRIC RELATIONSHIPS ............................................................ 165

APPENDIX D - CALCULATION OF THE CORRECTION FACTOR FOR THE

DENSIFICATION EFFECT ................................................................................... 172

APPENDIX E - PROGRAM DOCUMENTATION ...........................................................................173

APPENDIX F - RESULTS OF THE SIMULATION RUNS .......................................................211

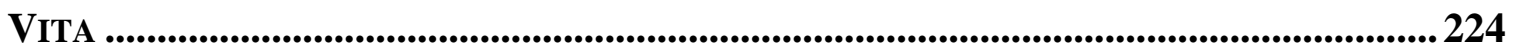

TABLE OF CONTENTS Vi 


\section{LIST OF TABLES}

6.1 Summary statistics of the measured physical properties of Appalachian hardwood species.

6.2 Summary and basic statistics of the experimentally determined shear strength values

6.3 Coefficients of determination provided by the various prediction models

6.4 Summary and basic statistics of the experimentally determined compression strength values

6.5 Summary and basic statistics of the experimentally determined compression MOE values

6.6 Coefficients of determination provided by the two prediction models for compression strength and MOE

6.7 Summary statistics of the experimentally determined dynamic MOE values. (Values are in $\mathrm{GPa}$ ).

6.8 Average absolute percent bias (AAPB) values associated with the three models.

6.9 Parameters of the first and second order regression equations, and the associated $\mathrm{r}^{2}$ values

6.10 Summary statistics of the physical properties of LVL and PSL

6.11 Flatwise and edgewise bending MOE of LVL and PSL ( $\mathrm{N}=20)$.

6.12 Summary statistics of the experimentally determined compression strength of LVL and PSL

6.13 Coefficients of determination provided by the various prediction models

6.14 Summary statistics of the compression MOE of the two composites

6.15 Summary statistics of the thickness of veneer sheets manufactured from the different species. (Thickness values are in $\mathrm{mm}$ ).

6.16 Summary statistics of the geometric parameters of LVL ...................................... 114

6.17 Summary statistics of the geometric parameters of PSL

7.1 Simulated geometric and physical properties of LVL and PSL (experimental LVL thickness statistics are also included)

7.2 Simulated edgewise and flatwise MOE of LVL and PSL

7.3 Simulated compression MOE of LVL and PSL in six directions.

7.4 The eight layup combinations; average edgewise and flatwise MOE 


\section{LIST OF FIGURES}

4.1 The orthotropy of solid wood shown in the principal material and global coordinate systems. Interpretation of grain angle $(\varphi)$ and ring angle $(\theta)$

4.2 The applied shear forces in the principal anatomical planes and the notation of corresponding shear stresses. $\mathbf{a}, \mathbf{b}$ - traditional shear tests, parallel to the grain; c, $\mathbf{d}$ - rolling shear.....

4.3 Interpretation and principle of the prediction process of the combined models .....31

4.4 Internal force conditions of an oblique specimen under compression

4.5 Interpretation of grain angle $(\varphi)$ and ring orientation $(\theta)$ of the applied compression load

5.1 Dimensions of the double-notched shear specimens 44

5.2 Schematic of the specimen manufacturing practice from prepared, straightgrained blanks.

5.3 Schematic of the shear testing apparatus and the experimental setup 46

5.4 Compression specimen manufacturing practice and the interpretation of $\varphi$ and $\theta$.......

5.5 Compression force application and the two-sided strain measurements 48

5.6 Schematic of the ultrasonic testing equipment and the experimental setup ............50

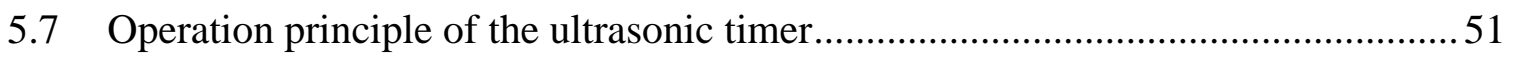

5.8 Schematic of the static tension test and the experimental setup ..............................53

5.9 The definition of orthogonal axes in LVL and PSL ...........................................5 57

5.10 Experimental setup of the composite bending tests................................................58

5.11 Geometric structure and stochastic parameters of LVL (a) and PSL (b) ................ 63

5.12 Digital images used for layer thickness measurements

6.1 Comparison of experimental and model predicted shear strength data of quaking aspen by orthotropic diagrams. 70

6.2 Comparison of experimental and model predicted shear strength data of red oak by orthotropic diagrams

6.3 Comparison of experimental and model predicted shear strength data of yellow-poplar by orthotropic diagrams 
6.4 Typical stress-strain diagrams

a. Traditional, parallel to the grain compression

b. Perpendicular to the grain compression

c. The effect of grain orientation

6.5 Orthotropic diagrams of compression strength and elasticity - quaking aspen ..... 81

6.6 Orthotropic diagrams of compression strength and elasticity - red oak ..................82 82

6.7 Orthotropic diagrams of compression strength and elasticity - yellow-poplar ....... 83

6.8 Characteristic failure modes of compression specimens ......................................... 85

6.9 Likelihood of shear failure in compression specimens

a. - critical shear stress contour of specimen $\varphi=45^{\circ}, \theta=15^{\circ} ; \sigma_{u}=23.24 \mathrm{MPa}$

b. - critical shear stress contour of specimen $\varphi=90^{\circ}, \theta=45^{\circ} ; \sigma_{u}=9.77 \mathrm{MPa} \ldots . .86$

6.10 Orthotropic dynamic MOE of quaking aspen structural veneer sheets

a. - experimental and model predicted values

b. - Average Absolute Percentage Bias associated with the different models

6.11 Orthotropic dynamic MOE of red oak structural veneer sheets

a. - experimental and model predicted values

b. - Average Absolute Percentage Bias associated with the different models

6.12 Orthotropic dynamic MOE of yellow-poplar structural veneer sheets

a. - experimental and model predicted values

b. - Average Absolute Percentage Bias associated with the different models

6.13 Linear (a) and quadratic (b) relationship between the static and dynamic

MOE of the three Appalachian species measured at various grain angles.

6.14 Relationship between dynamic MOE increase and densification for aspen (a), red oak (b) and yellow-poplar (c)

6.15 Relationship between the static MOE increase and densification for yellow-poplar veneer strands with longitudinal grain orientation.

6.16 Comparison of the static and dynamic densification curve of yellow-poplar. Modified dynamic densification curve.

6.17 Relationship between the edgewise and flatwise bending MOE of LVL (a) and PSL (b)

6.18 Orthotropic diagrams of the shear strength of LVL and PSL

6.19 Statistical distribution and probability density function of the veneer thickness for the three species

6.20 Layer thickness distribution throughout the thickness of LVL 
6.21 Statistical distribution and probability density function of the geometric

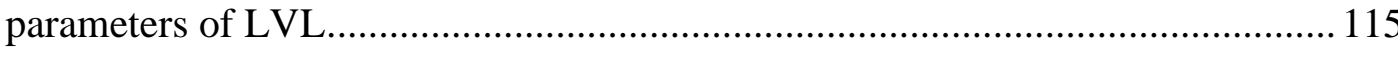

6.22 Statistical distribution and probability density function of the geometric parameters of PSL

7.1 The systematic arrangement of the strands in PSL.............................................. 121

7.2 Strands situated at various positions in a PSL cross-section ................................. 123

7.3 Experimental and simulated cross-sections of LVL (a) and PSL (b) ................... 131

7.4 Comparison of the experimental and simulated flatwise and edgewise

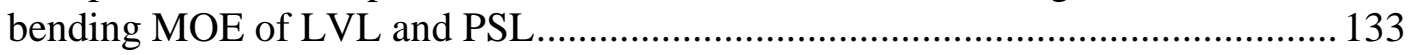

7.5 Comparison of the experimental and simulated compression MOE of LVL and PSL in the six simulated direction .............................................................. 136

7.6 Orthotropy diagrams created from the experimental and simulated compression MOE of the composite materials

7.7 Flatwise and edgewise bending MOE of simulated LVL beams with various

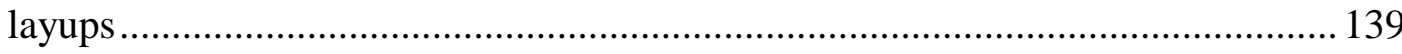

7.8 The effect of the variance of $\alpha$ and $\beta$ on the flatwise (a) and edgewise (b) bending MOE of PSL 


\section{LIST OF SYMBOLS AND ABBREVIATIONS}

$\begin{array}{ll}b_{0} & \text { - } y \text {-intercept in polynomial functions } \\ b_{1} & \text { - coefficient of } x \text { in polynomial functions } \\ b_{2} & \text { - coefficient of } x^{2} \text { in polynomial functions } \\ l & \text { - longitudinal axis of a strand } \\ n & \text { - power in the modified Hankinson formula } \\ m & \text { - angle resolution for orthotropic investigations } \\ o & \text { - cross-sectional orientation axis of a strand } \\ \mathrm{r}^{2} & \text { - coefficient of determination } \\ \mathrm{s} & \text { - standard deviation } \\ t_{o} & \text { - original veneer/strand thickness } \\ t & \text { - layer or projected strand thickness } \\ v & \text { - propagation velocity of sound in a material } \\ u & \text { - number of strands per in }{ }^{2} \text { of cross section in PSL } \\ x & \text { - longitudinal direction in the composite lumber } \\ y & \text { - the cross-sectional direction parallel with the constitution orientation in } \\ z & \text { composite lumber } \\ & \text { - the cross-sectional direction perpendicular to the constitution orientation in } \\ & \text { composite lumber }\end{array}$

A - cross-section of a simulated composite beam

$A_{i} \quad$ - cross-section of a constituent in a simulated composite beam

$E \quad-$ Modulus of Elasticity (MOE)

$\bar{E} \quad$ - mean MOE value

$\hat{E} \quad$ - predicted MOE value

$E_{\text {edge }} \quad$ - Edgewise bending MOE

$E_{\text {flat }} \quad$ - Flatwise bending MOE

$E_{i} \quad-$ simulated MOE of the $i^{\text {th }}$ constituent in LVL or PSL

$E_{I} \quad-\mathrm{MOE}$ in an anatomical direction $(I=\mathrm{L}, \mathrm{R}, \mathrm{T})$

$E_{i}^{j} \quad$ - MOE measured at $\varphi=i ; \theta=j$ 


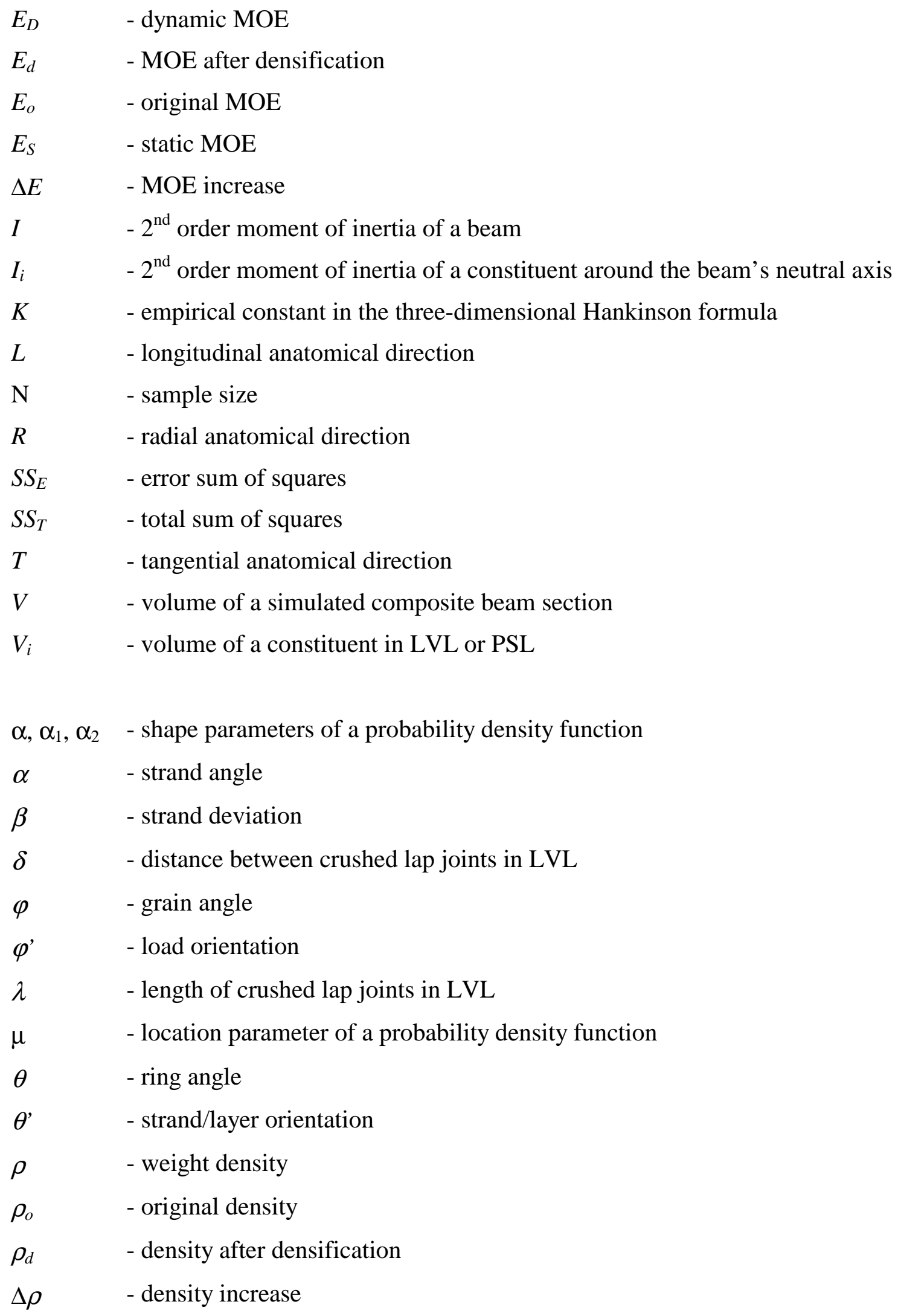




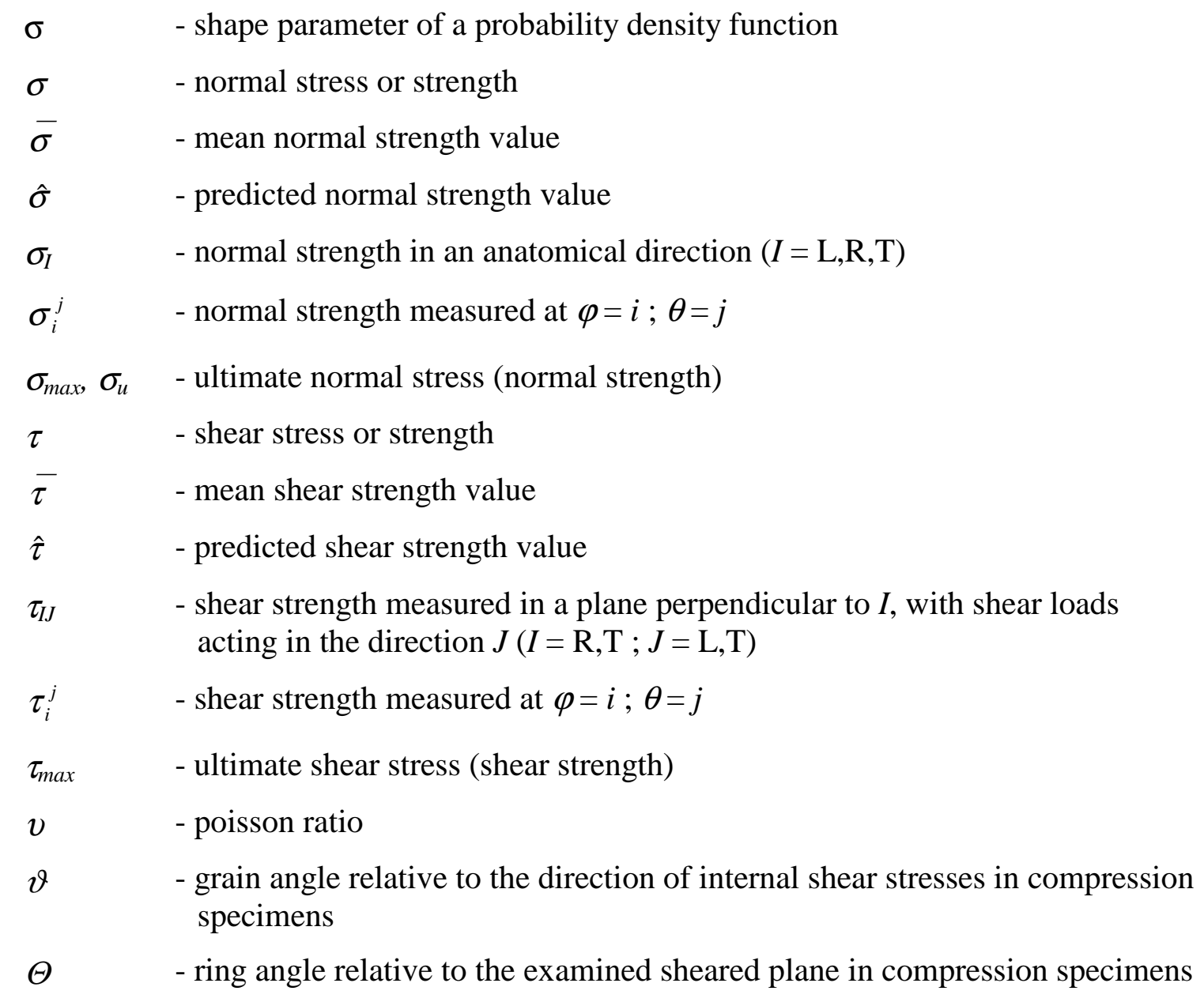

$\begin{array}{ll}\text { AAPB } & \text { - Average Absolute Percentage Bias } \\ \text { ANOVA } & \text { - Analysis of Variance } \\ \text { ASTM } & \text { - American Society for Testing and Materials } \\ \text { HDD } & \text { - Horizontal Density Distribution } \\ \text { LSL } & \text { - Laminated Strand Lumber } \\ \text { LVL } & \text { - Laminated Vener Lumber } \\ \text { MC } & \text { - Moisture Content } \\ \text { MOE } & \text { - Modulus of Elasticity } \\ \text { MSS } & \text { - Maximum Shear Strength } \\ \text { MTS } & \text { - Material Testing Systems } \\ \end{array}$




$\begin{array}{ll}\text { PVAc } & \text { - Polyvinyl Acetate } \\ \text { R\&D } & \text { - Research and Development } \\ \text { RCB } & \text { - Randomized Complete Block } \\ \text { RH } & \text { - Relative Humidity } \\ \text { SG } & \text { - Specific Gravity } \\ \text { STD } & \text { - Standard deviation } \\ \text { VDD } & \text { - Vertical Density Distribution }\end{array}$




\section{INTRODUCTION}

Before the $20^{\text {th }}$ century, wood was the almost exclusive building material of residential and commercial structures in North America. Abundant natural resources provided ample raw material for dams, bridges, log houses, etc. By the turn of the century the situation had changed, and the finiteness of these resources became apparent. The concept of light frame building has evolved, through balloon framing to the more contemporary platform framing used today in residential construction.

Floors and walls in light frame structures typically consist of a network of loadbearing elements (frame) covered to provide a platform surface (sheathing.) Historically, the raw material for both frame and sheathing was solid sawn lumber. In sheathing applications, wood based composite panels like plywood and - later - flakeboard, were soon substituted for solid wood. These products represent a fuller utilization of the available logs, and have other advantages in sheathing uses, such as their larger dimensions, improved uniformity of characteristics and their load-bearing capacity in two directions.

The replacement of solid wood in framing applications was more difficult. The favorable specific mechanical properties of wood make it a tough competitor for inorganic materials (steel, concrete) and wood based composite products. It was not until the last three decades of the $20^{\text {th }}$ century that the first effective composite substitute emerged in the US market. Presently, there are four types of wood based composites used for framing: Laminated Veneer Lumber (LVL), Laminated Strand Lumber (LSL) and Parallel Strand Lumber (PSL), and engineered wood I-Joists. 
Although structural composite lumber products gained a substantial share of the market for construction lumber, their production cost is relatively high. Despite manufacturers' efforts, the complex production procedure and the price of adhesives used severely limit price reduction opportunities. The only feasible alternative is increasing the perceived value of these products. Manufacturers are constantly seeking alternative raw materials and innovative designs to provide superior performance and consistency at the same cost. The goal of the present study was to aid these efforts through the development of simulation models that can be used to explore new designs or raw material sources in the manufacture of composite lumber products.

\subsection{Laminated Veneer Lumber (LVL)}

LVL is a structural lumber manufactured from veneers laminated into a panel with the grain of all veneers running in the same direction. The resulting material is usually 19 to $45 \mathrm{~mm}$ (3/4 to $1-3 / 4 \mathrm{in})$ thick, 0.6 to $1.2 \mathrm{~m}$ wide, and ripped to common lumber widths of 38 to $290 \mathrm{~mm}$ (1-1/2 to 11-1/2 in) or wider (Wood Handbook 1999). LVL is also known as Parallel Laminated Veneer (PLV) or Press-Lam.

The first laminated veneer structure of this type was proposed by Luxford (1944), for constructing high-strength wood aircraft members. The development of LVL for commercial use started in 1967 (Kunesh 1978.) Early studies typically used thick veneers (1/4 in or thicker) to construct and examine laminated structures (Koch 1967; FPL-PressLam Research Team 1972, Bohlen 1972, Jung 1984.) Today, the majority of LVL is made by continuously laminating rotary-peeled, $3.2 \sim 2.5 \mathrm{~mm}(1 / 8 \sim 1 / 10$ in $)$ thick veneer 
sheets. The traditional raw materials for LVL manufacture are coniferous species, like Douglas-fir (Pseudotsuga mensiesii (Mirb.) Franco) and southern pine (Pinus spp.) In the 90's manufacturers started using yellow-poplar (Liriodendron tulipifera L.), too. (Vlosky et al. 1994.)

LVL has several advantages compared to solid wood lumber (based on FPLPress-Lam Research Team 1972, Laufenberg 1983 and Vlosky et al. 1994):

- Better yield. Research has shown that "using nominal or dressed dimensions and assuming lumber recovery from the core, LVL yielded more than 47 percent more than sawn lumber" (Laufenberg 1983.) Although this is not clearly manifested in product price, this represents an environmentally sounder practice, which, through environmental marketing, can provide competitive advantage, for the producer;

- Mechanical properties. Anatomically inherent defects of solid wood (knots, sloping grain, etc.) are dispersed in LVL. This leads to increased strength and less variation in mechanical and physical properties. As a consequence, allowable stress values increase. In addition, non-destructive grading of veneers allows manufacturers to engineer the mechanical parameters of their product;

- Weight. Because of the above facts, lighter beams can be used, which speeds up construction and alleviates job site injuries;

- Size. Using a continuous process (most widespread presently), very long and deep beams can be produced at unchanged costs. The price of solid wood lumber increases with length and width, and availability of lumber is limited by the length and diameter of the saw logs;

- Reduced job site waste;

- Less frequent customer complaints. 
Initially, LVL manufacturers aimed at the same market niche occupied by high quality lumber, endeavoring to keep prices competitive. In time it became apparent that LVL manufacture would remain expensive, but that the product can be superior to solid wood in many applications. Since that time, manufacturers are trying to establish their product as a value-added commodity both through $R \& D$ and marketing communication. LVL is a relatively new product, still at the 'growth' phase of its product life-cycle. Margins are still attractive, and production is likely to increase for a long time yet (Vlosky et al. 1994).

\subsection{Parallel Strand Lumber (PSL)}

PSL is a structural composite lumber made from wood strand elements with the wood fiber oriented primarily along the length of the member. The strands are coated with a waterproof structural adhesive, and fed, in a highly oriented manner, into a special press frame that uses microwave technology to cure the resin. The pressing operation results in high levels of densification. (Wood Handbook 1999)

PSL has originally developed as an alternative way of utilizing veneers that - due to defects created during growth, storage or handling - did not qualify for plywood or LVL manufacture. Its physical and mechanical properties are, however, no worse (often better) than those of LVL. Mechanical properties of PSL are just as consistent as those of LVL (Kunesh 1978, Rammer and Zahn 1997). PSL is an increasingly popular composite lumber product, that is already a significant competitor of LVL and solid wood. Presently, there is only one manufacturer of this product. 
The advantages and disadvantages of PSL are similar to those of LVL. Additional advantages include its appearance that many people find attractive. PSL is available in practically infinite length, while the only limitation on the cross-sectional dimension is the size of the press frame. LVL, in contrast, is typically available as $2 x$ nominal crosssections, or smaller.

\subsection{Simulation modeling}

The purpose of applied sciences is to gain better understanding of real-world facilities or processes. The traditional and most straightforward method to achieve this is experimenting with the real-life entity. In some situations, however, such investigations entail serious difficulties. Examination of real-life entities is often very costly or too disruptive to the facility or process in question. In other instances, the investigation may be very time-consuming. The subject of the examination might not even exist; nevertheless, it might be important to study its characteristics and interaction with its hypothetical environment. In these cases, the investigator might create a simplified representation of the system to explore certain properties at lower costs, faster rates or without disrupting the original entity. This technique is called modeling.

Researchers may use various types of models, depending on the task at hand. These include physical models, mathematical models with analytical solutions, and simulation models (Law and Kelton 1991.) Simulation models are useful if the entity to be modeled (called system) contains probabilistic elements. An example is a grocery store, where the number of customers arriving in a certain period of time, the number of 
customers in the store at any time, supplies, etc. are not predictable with absolute certainty.

Wood science applications include many areas - both in material science and process control - where simulation modeling can be serviceable. In recent years, scientists employed simulation in various fields of study. These models include a wide range of applications, e.g. composite production processes (Kurse et al. 1997), hot pressing (Humprey and Bolton 1989, Lenth and Kamke 1996b), furniture rough mill operation (Anderson, 1983), laminated wood panel warping (Suchsland and McNatt 1986), etc.

Wood based composite production and properties involve many probabilistic components. Real-life experimentation with these composites, which involves altering manufacturing parameters of automated production lines, is very costly, time-consuming and disruptive. Simulation modeling is an excellent tool to investigate the manufacture, composition, physical and mechanical properties of these materials, and has been widely utilized by various researchers, as demonstrated in section 3.3. 


\section{OBJECTIVES AND DISSERTATION STRUCTURE}

\subsection{Objectives}

The objective of the research described in this dissertation was to develop and validate simulation models that can estimate the mechanical properties of Laminated Veneer Lumber (LVL) and Parallel Strand Lumber (PSL). This included the following tasks:

1. Building input databases for the model, including the orthotropic mechanical properties of the raw material and the parameters of composite geometry.

2. Examining the effect of the manufacturing process on the constituents.

3. Assessing the composites' mechanical properties experimentally.

4. Modeling the bending and orthotropic compression Modulus of Elasticity (MOE) of the composites using deterministic and stochastic simulation, and validating the models by comparing simulation results to the experimentally obtained values.

\subsection{Structure of the dissertation}

Chapter 3 summarizes the results of former investigations concerning the orthotropic mechanical properties of solid wood, the effect of the manufacturing practice on the constituents and the results of simulation studies that modeled the manufacture and properties of wood based composites. Chapter 4 provides background information that was used in exploring the orthotropic nature of solid wood's mechanical properties, and provides the theoretical basis for the simulation models. 
Chapter 5 introduces the materials and experimental methods used for the investigation of the mechanical properties of solid wood and those of LVL and PSL. It also describes the practice of assessing the composites' geometric properties that were necessary for the prediction models. The results of these investigations are discussed in Chapter 6.

Chapter 7 contains details of the developed models that predict the bending and compression MOE of the composite lumber products. This chapter includes the experimental validation of these models versus the experimentally measured properties of LVL and PSL, and demonstrates the capabilities of the models to predict the properties of composites that use alternative raw materials or design features.

Finally, chapter 8 concludes the dissertation, and chapter 9 provides recommendations for further work that might improve and extend the predictive capacity of the models. 


\section{LITERATURE REVIEW}

\subsection{Orthotropic strength and elasticity of solid wood}

\subsubsection{Orthotropy of shear strength}

True shear strength is one of the most difficult characteristics to measure. Creation of the pure shear stress state is a real challenge. Furthermore, the always present normal stresses combined with the inherent anisotropy of wood make the strength determination uncertain. Several publications have dealt with the improvement of shear strength assessment. One of the most comprehensive studies on this topic was provided by Yilinen (1963). The author investigated and critically reviewed several standardized shear testing methods. He concluded that the majority of block shear tests usually underestimate the true shear strength of solid wood.

The standard ASTM block shear test has received much criticism for not providing pure shear load on the specimens. A number of researchers addressed this problem and some also proposed alternative solutions. Norris (1957) recommended the panel shear test, and Liu (1984) suggested the adaptation of a device, proposed by Arcan et al. (1978) for wood. The drawback of these tests is that they involve complicated specimen preparation and testing procedures. Lang (1997) proposed a new device for shear strength assessment of solid wood. The advantages of the described testing apparatus are the smaller specimen size, alleviation of normal stresses and acceptable agreement with shear strength values obtained by the ASTM method. 
The majority of previous research projects have focused on the shear strength of solid wood parallel to the grain. Limited publications are available that address the anisotropy of wood in shear strength assessment.

The first formula that described the strength anisotropy of wood is the wellknown Hankinson's formula (Hankinson 1921). It was developed empirically from compression tests. This equation describes the effect of grain-orientation changes on the measured properties. Many researchers examined the validity of this formula finding that it fits experimental data well (Goodman and Bodig 1972; Bodig and Jayne 1982). However, the equation was deemed to provide adequate predictions only for compression and tension strength as well as moduli of elasticity. Kollman and Cote (1968) proposed some changes to the formula. Kollman (1934) used an experimentally determined power that provided better approximation of the direction dependent strength and elastic properties. The first attempt to describe the orthotropy of shear strength was made by Norris (1950). He applied the general Henky - von Mises theory to orthotropic materials. Although in his study the predicted shear strength values agreed reasonably well with experimental data for structural plywood, the approach has received criticism from others (Wu 1974; Cowin 1979). Over the decades, with the advancement of man-made composites, ample research has been devoted to explore the strength and elasticity of anisotropic materials. Many of these results and theories may be applied to wood with care.

Ashkenazi (1978) used the tensor theory for describing the anisotropy of wood and wood-based composites. In an earlier work he measured the shear strength of pine at various grain angles (Ashkenazi 1959). His results were unusual in that shear strength 
showed maximum values at approximately $15^{\circ}$ grain orientation, rather than in the longitudinal direction. Cowin (1979) stated that a quadratic form of the Hankinson's formula describes Ashkenazi's data reasonably well. The proposed model, however, can not describe the shear strength maximum at $15^{\circ}$ grain orientation. Liu and Floeter (1984) measured the shear strength of spruce at $0^{\circ}, 30^{\circ}, 60^{\circ}$ and $90^{\circ}$ grain angles with the special device described by Arcan et al. (1978) designed to provide uniform plane stress. Their results agreed well with the theory of Cowin (1979).

Some other researchers incorporated the effect of ring orientation in their works. The experiment of Bendsten and Porter (1978) included ring-angle, but only as a blocking factor, its effect was not of interest. Okkonen and River (1989) examined the effect of radial and tangential ring orientation on the shear strength in the longitudinal direction. They concluded that Douglas-fir had higher strength when the orientation of the sheared plane was radial, while oak and maple were stronger in the tangential direction. Riyanto and Gupta (1996) tried to establish a relationship between ring angle and shear strength parallel to the grain. Using a completely randomized design, they found that ring angle had very little effect on the shear strength of Douglas-fir and Dahurian larch. Rather, the specific gravity, the percentage of latewood and the number of rings per inch were much more deterministic factors. Szalai (1994) provided an integrated approach that tackles both ring and grain angle orientation. A general equation, derived from tensor analysis, can determine the shear strength at any given ring and grain angle combination. 


\subsubsection{Orthotropy of compression strength and elasticity}

The orthotropy of uniaxial stresses like compression and tension have received more attention than did shear stresses, both because of their ease of assessment and importance in practical applications. Much research effort was concentrated in this area, but most of the works focused on the effect of grain angle or ring angle, separately.

As mentioned in the previous section, the most well known model to describe the effect of sloping grain on compression properties is Hankinson's formula (Hankinson 1921). Radcliffe (1965) investigated the accuracy of the equation, comparing its predictions to theoretical values of MOE that were derived from the relationships of orthtotropic elasticity. He showed that Hankinson's solution is quite accurate in the $L R$ plane, while in the $L T$ plane around $25^{\circ}$ grain inclination it may underestimate the MOE by $30 \%$. Other researchers also verified the validity of this model (Goodman and Bodig 1972, Bodig and Jayne 1982). Kollmann and Cote (1968) suggested some modifications to the original formula, based on the results of Kollman (1934). Cowin (1979) gave a good overview of these developments, and concluded that the valid formula should be the one Hankinson originally proposed. Some published research works claimed that another version, the so-called Osgood formula, approximates better the effect of sloping grain than the Hankinson's equation (Kim 1986, Bindzi and Samson 1995). The Osgood formula, that is also empirical, is given as follows:

$$
m=\frac{p q}{q+(p-q) \sin ^{2} \varphi\left(\sin ^{2} \varphi+a \cos ^{2} \varphi\right)}
$$

Where $m, p$ and $q$ are the compression properties at grain angles $\varphi, 0^{\circ}$ and $90^{\circ}$, respectively. Constant $a$ is a species-specific coefficient that should be determined 
experimentally. However, no extensive validation of this model was reported in the literature (Kim 1986).

Transverse compression has received much attention, too. Bodig (1965), Kunesh (1968) and Bendsten et al. (1978) provided more in-depth analysis of the question. Ethelington et al. (1996) incorporated variation of ring orientation in their work, and concluded that it had significant effect on the compression strength perpendicular to the grain.

The exact determination of the strength perpendicular to the grain is practically impossible because of the practical incompressibility of the wood substance. The ASTM D 143 - 83 standard requires that the test shall be discontinued after 0.1 inch crossheaddisplacement. This procedure was developed to evaluate the reaction force supporting capacity of solid wood joists. Consequently, there is no standard testing method that regulates the exploration of orthotropy in compression. However, there are several theories for predicting the failure envelope of solid wood and/or wood-based composites. Usually these approaches are based on six-dimensional tensor analyses like the Tsai-Wu strength criterion (Tsai and $\mathrm{Wu}$ 1971) that was developed two decades ago for homogeneous, orthotropic materials such as glass or carbon fiber and epoxy composites. The fiber direction in these synthetic composites is better controlled and the materials are transversely isotropic (i.e., identical strength and elastic properties in any directions perpendicular to the fiber). Thus, such analyses can be successfully used in exploring the strength orthtotropy of relatively homogeneous materials as demonstrated through an analysis of paperboard by Suhling et al. (1985). 


\subsubsection{The orthotropy of tensile elasticity}

Tensile properties of wood are more difficult to assess than compression strength and elasticity. The difficulties are even greater when sloping grain is involved. Limited research has been done on the tensile orthotropy of wood. Gerhards (1988) examined the effect of sloping grain on the tensile strength of Douglas-fir. At small angle deviations (less than $20^{\circ}$ ) he found the modified Hankinson's formula (Kollman and Cote 1968) to provide acceptable fit. In another work (Woodward and Minor 1988) that included the full grain orientation range, the authors found the same theory to work well, but provide worse prediction than a Hyperbolic formula. Pugel (1990) developed an angle-to-grain tensile setup for thin specimens. Tensile test results of Douglas-fir and southern pine, measured using his setup, showed reasonable agreement with the original Hankinson's formula. These studies dealt with the tensile strength only.

Nondestructive testing is a simple and inexpensive alternative to static tests. Its advantages are obvious: the specimen is not destroyed during the test, which is usually fast and cheap, and nondestructive evaluation is often much less complicated than the static test. Vibration methods are particularly suitable for quantitative, as well as qualitative evaluation of materials. The relationship of vibration properties to elastic characteristics was recognized as early as 1747 by Riccati. Researchers started to apply this relationship for wood in the early 1950's (Pellerin 1965.)

Vibration methods include two subtypes: transverse and longitudinal (stresswave) vibration. According to theory, measured transverse vibration frequency and wave propagation velocity are related to the bending and uniaxial MOE, respectively. Many studies verified these relationships experimentally, typically with excellent results. 
Researchers also endeavored to find empirical correlation between vibration and strength properties. Many considered the damping characteristics of wood to be promising, but experiments were not invariably successful. Pu and Tang (1997) gave an excellent overview of the research conducted in this area.

The application of static tension tests to veneers is especially limited due to their small thickness. The mechanical properties of veneer, can be very different from those of the wood it originated from, and assessment of veneer properties is sometimes desirable. This is an area where nondestructive testing (specifically, stress-wave timing) is very helpful. There are two areas where vibration testing of veneers can be particularly useful: 1.) relating the mechanical properties of logs to those of the veneer peeled from them (Ross et al. 1999, Rippy et al. 2000), and 2.) veneer classification prior to Laminated Veneer Lumber manufacture, to engineer or improve the consistency of the product's end properties (Koch and Woodson 1968, Jung 1982, Kimmel and Janiowak 1995, Shuppe et al. 1997.) The latter gained practical application, too, and a commercial tool is now widely used for classifying veneer sheets according to their stress-wave characteristics (Sharp 1985.)

Other studies about veneer testing by stress-waves include that of Jung (1979), who presented a comprehensive study concerning stress-wave application on veneers. He examined the potential of this technique to detect knots and slope of grain, and investigated the effect of specimen size and different measurement setups. Hunt et al. (1989) correlated the tensile and stress-wave MOE of veneer, with acceptable results. Most recently, Wang et al. (2001) investigated the potential of two stress-wave 
techniques to detect lathe checks and knots in veneer. Stress wave propagation parameters were sensitive of defects, when measuring perpendicular to grain.

Few studies dealt with the effect of sloping grain on nondestructive testing parameters. Kaiserlik and Pellerin (1977) attempted to predict tensile strength of woods containing sloping grain. Armstrong et al. (1991) studied the effect of grain orientation on the wave-propagation velocity in various species. They concluded that, out of three equations, the modified form of the Hankinson formula (Kollman and Cote 1968) provided best fit to the data, but warned that some limitations may question its appropriateness for some applications. Divos et al. (2000) used ultrasonic propagation velocity and attenuation parameters to predict grain slope. They showed that both ultrasonic velocity and the magnitude of the first received amplitude are good indicators of grain deviation. Attenuation is better to detect small grain deviations, while propagation velocity - which is a function of the direction-dependent MOE - is a better estimator for the entire grain orientation range. Jung (1979) examined (among other factors) the effect of sloping grain on the stress-wave characteristics of veneers. He found that at small angles there is little change in stress-wave velocity, but at slightly higher orientations velocity decreases rapidly. There appears to be no study in the literature that uses vibration methods to describe the relationship between MOE and grain alignment in wood. 


\subsection{Modification of constituents' properties during manufacture}

The raw materials of wood based composites undergo a number of changes during processing. These effect the mechanical properties of the constituents.

Veneer peeling affects the mechanical properties of the constituents through splitting and checking on the backside of the veneer. Further modification occurs during glue application and hot pressing. Some of the adhesive applied to the constituent will penetrate its surface layer, and modify its mechanical properties.

Bodig and Jayne (1982) gave a detailed description of the phenomenon called polymeric impregnation. Based on the results of Langwig et al. (1968) and Bryant (1966) they concluded that phenolic resin tends to increase bending and compression properties, but reduce tension strength, toughness and dynamic properties. Application of the law of mixtures to impregnated wood, taking the void volume in account, yields an equation that shows that the effect of the polymer on the MOE of the compound is additive. Experimental validation showed this theory to provide reasonable prediction for impregnated wood (Siau et al. 1968, Taneda et al. 1971).

In composite simulation, glue penetration has a double role. The more adhesive the constituents take up, the less remains for bonding. On the other hand, impregnation influences the mechanical properties, as discussed above. Triche and Hunt (1993) demonstrated the latter effect by a finite element study that incorporated a wood-resin interface layer, which increased MOE. The model predicted experimental tensile MOE and ultimate stress reasonably well. 
Densification is another consequence of hot pressing. Establishing close contact between the constituents - which is imperative for good bonding - requires pressure application. The necessary pressure is higher in randomly aligned composites, and lower, but still significant in systematically aligned ones. (Dai and Steiner 1993). In either case, some densification results.

The effect of densification on mechanical properties is twofold. The original bulk of cell wall material is squeezed into a smaller volume. This means that there will be more material to resist stresses, which will improve both the strength and the elastic properties of the constituent. Xu and Suchsland (1998b) modeled the MOE of composite panels based on this assumption. According to their model, MOE improves proportionally to density-increase.

Relationships between density and MOE of solid wood seem to bear out the above theory. Bodig and Jayne (1982) presented the following equation to predict mechanical properties from density:

$$
Y=a \rho^{b},
$$

where $Y$ is the characteristic in question, $\rho$ is the density and $a$ and $b$ are experimental constants. Markwardt and Wilson found $b$ to be one for bending and compression MOE. In another study (Bodig and Goodman 1972), for the MOE of softwood and hardwood in different anatomical directions, $b$ values differed from, but were close to, unity.

On the other hand, fractures and inelastic strains may develop within the elements, reducing their inherent strength and stiffness (Palardy et al. 1989). This alleviates the above mentioned improvement, but the overall effect tends to be positive, especially at higher levels of densification (Price 1976). Geimer et al. (1985) analyzed 
the effect of densification using various pressing parameters, using micromechanical tools. They concluded that high press temperatures cause less damage in the veneer, because of plastification, and are more favorable in terms of mechanical properties.

\subsection{Wood composite modeling}

Constituent manufacture, mat formation and consolidation are complex procedures that involve variables that, despite efforts to control them, are influenced by several random factors. The resulting products have many random characteristics, as well. It is not surprising, that many researchers used various modeling techniques to further the understanding of wood-based composites.

Modeling physical and mechanical properties requires a thorough understanding of the spatial structure of the composites. An early simulation model described the structure of paper as consisting of several layers of fibers and interfibrillar spaces or pores (Kallmes and Corte 1960, 1961). This work provided a basis to developing a mathematical model that describes randomly packed, short-fiber-type wood composites (Steiner and Dai 1993, Dai and Steiner 1994a, 1994b). This simulation is based on the observation that flake positions in a layer are driven by Poisson processes. As a consequence, point mass density and overall mat thickness will have Poisson distributions, too. The results of this investigation were used in a Monte Carlo simulation program that can model different types of mats, and analyze them for various important geometric characteristics (Lu et al. 1998). The program can also determine the effect of sampling zone size on the measured density distribution. Harris and Johnson (1982) dealt 
with the characterization of flake orientation in flakeboards. They pointed out that unbounded distributions are not appropriate for this purpose and suggested a bounded distribution to provide angles between 0 and $\pi$.

Some researchers attempted to provide detailed explanation and simulate certain aspects of particle mat behavior during consolidation. Suchsland (1967) summarized the mat formation, heat- and moisture movement and stress-behavior of particleboard mats. He provided an explanation for the formation of horizontal and vertical density distributions, and showed how pressing parameters influence the latter. Humprey and Bolton (1989) made an in-depth analysis of the multidimensional unsteady state heat and moisture transfer during hot pressing. They built a model, based on a modified finite difference approach, that could predict temperature, moisture content, vapor pressure and relative humidity in different layers of a mat.

Several works dealt with the compression behavior of flake mats throughout the pressure cycle. Dai and Steiner (1993) developed a theoretical model to describe the compression response of randomly formed wood flake mats. Their predictions agreed with experimental results reasonably well. Two further models, using somewhat different approaches to mat structure and stress-strain relationship characterization, provided improved estimation. One of these incorporated the effect of flake bending during press closure (Lang and Wolcott 1996a, 1996b) while the other used theories of cellular materials (Lenth and Kamke 1996a, 1996b.) It has been proposed that a combination of these two models characterizes the entire stress-strain curve best.

Physical and mechanical properties of wood based composites are closely related to density. Vertical and horizontal density distribution (VDD and HDD) generated much 
research interest. Suchsland and Xu $(1989,1991)$ built physical models to examine the effect of HDD on thickness swelling and internal bond strength. They concluded that the durability of flakeboard is substantially effected by the severity of the horizontal density distribution. Xu and Steiner (1995) presented a mathematical concept for quantifying the HDD. Another study (Wang and Lam 1998) linked a simulation program with an experimental mat through a robot system that deposited flakes in the simulated positions. Simulated and actual HDD showed good agreement.

Harless et al. (1987) created a very comprehensive simulation model that can regenerate the VDD of particleboard as a function of the manufacturing process. Other research in this area includes characterization of VDD using a trigonometric density function (Xu and Winistorfer 1996), and a simplified physical model to examine how the number of flakes, face flake moisture content and press closing time affects VDD (Song and Ellis 1997.)

Zombori (2001) created a series of linked simulation and finite element models that could, in turn, recreate the geometric structure, compression behavior, and heat and mass transfer of oriented strand board. These models could predict the inelastic stressstrain response, environmental conditions, moisture content and density at different points within the panel. His results - some of which are applicable to other composites, like particleboard, too - were in reasonable qualitative agreement with reality, although their quantitative accuracy was sometimes questionable.

Simulation studies have dealt with the mechanical properties of wood based composite panels. Most of these models were created by $\mathrm{Xu}$ and Suchsland. They simulated the linear expansion of particleboard (1997), followed up by a study discussing 
the effect of out-of-plane orientation (1998a). In these works, they made use of the offaxis MOE, determined by the Hankinson formula. They used the findings of these studies in a later model (1998b), to simulate the uniaxial MOE of composites with uniform VDD, based on the total volumetric work. This model accounted for the effect of densification and that of particle orientation, and the authors made observations about the effect of other factors (like glueline quality and manufacturing treatments) on the simulation. $\mathrm{Xu}$ (1999) improved this simulation to model the effect of vertical density profile on the bending MOE of composites, using the laminate theory. He described the VDD by the trigonometric function provided by $\mathrm{Xu}$ and Winiesdorffer (1996), and found that maximum MOE results when peak density is some distance from the surface. The validity of this observation, however, depends on the validity of the VDD function used. The above model was applied to evaluate the effect of percent alignment and shelling ratio on the MOE of OSB (Xu 2000) Simulation results agreed well with experimental data in literature.

Triche and Hunt (1993) modeled parallel-aligned wood strand composites using finite element analysis. They created small scale parallel-aligned strand composites, that can be regarded as physical models of LVL or PSL. The applied finite element model accounted for the effect of densification, adhesive penetration and crush-lap joints, and estimated the tensile strength and MOE of the specimens with excellent accuracy. In a very recent study, Barnes (2001) modeled the strength properties of oriented strand products. He introduced the concept of stress transfer angle to assess the effect of strand length and thickness on the mechanical properties of composites. He found good 
agreement between experimental and model-predicted MOE, MOR and tensile strength values.

Wood based composite lumbers, such as LSL, LVL or PSL, are relatively new products that generated less research interest than did composite panels. Many findings of the above papers can be applied to these products with care. In the meantime, available literature does not seem to contain simulation studies that are directed specifically towards modeling the geometric structure and mechanical properties of these composites. 


\section{THEORETICAL BACKGROUND}

\subsection{Orthotropic strength and elasticity}

\subsubsection{Orthotropy of shear strength}

The orthotropic nature of solid wood is usually depicted in a three-dimensional Cartesian coordinate system as shown on Figure 4.1. The principal directions of the material coordinate system are noted as L, R and T, longitudinal, radial and tangential directions, respectively. If an aligned global coordinate system $\left(x_{i} ; i=1,2,3\right)$ is

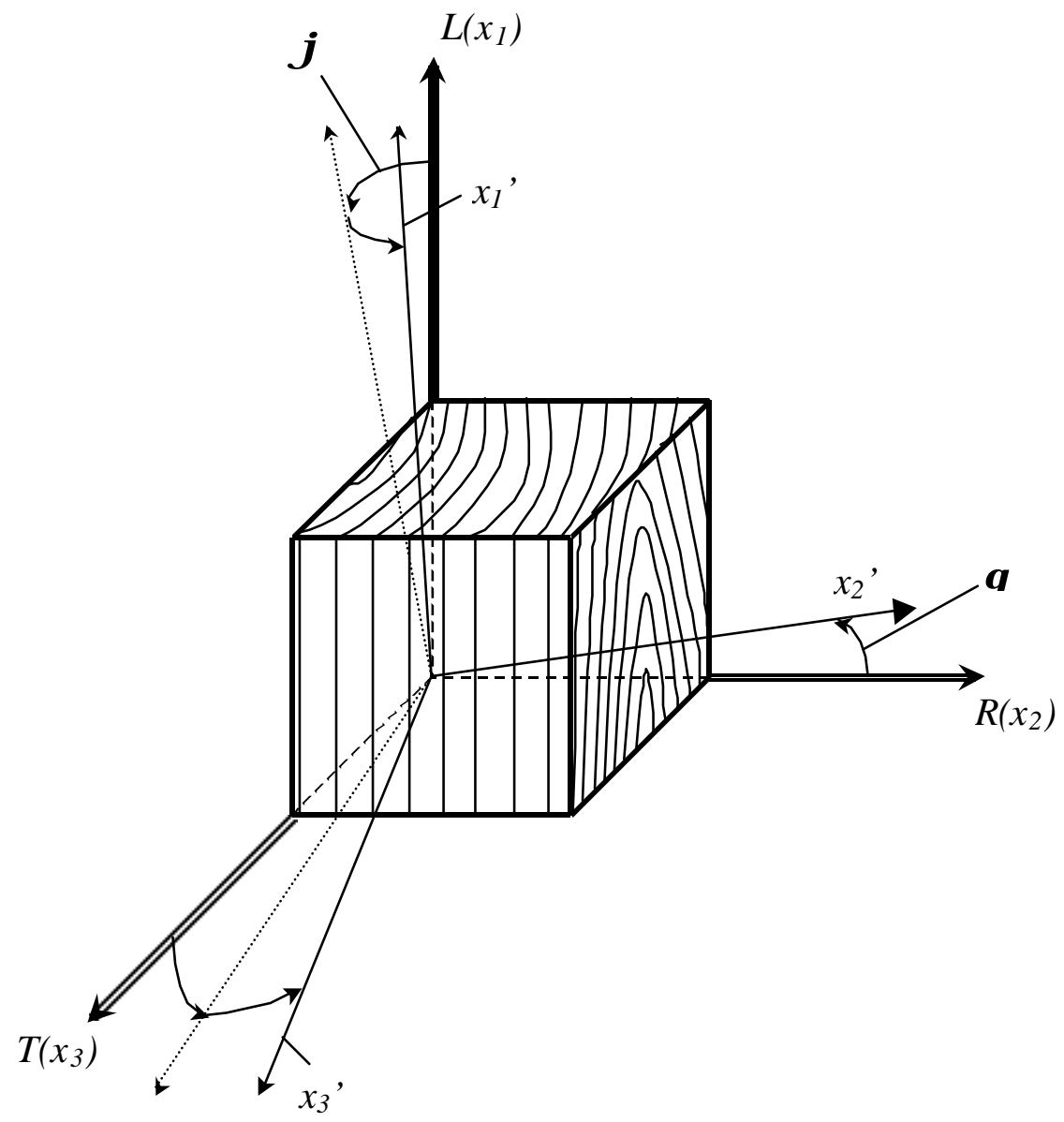

Figure 4.1 - The orthotropy of solid wood shown in the principal material and global coordinate systems. Interpretation of grain angle $(\varphi)$ and ring angle $(\theta)$ 
systematically rotated around the $R$ and $L$ axes, the angles between the axes of $L, R, T$ and $x_{i}{ }^{\prime}(i=1,2,3)$ systems correspond to the grain and ring orientation of solid wood relative to the global coordinate system as marked on Figure 4.1. Note that the $x_{1}$ ' $x_{3}$ ' plane is always parallel to the grain. If shear forces are acting in this plane and the direction of the applied forces is $x_{1}$, the orthotropy of shear strength can be investigated as a function of grain and ring angle. Using the described rotation, block shear specimens can be machined and tested. Such specimens are shown on Figure 4.2 representing the shear strength $(\tau)$ measurements in the principal material directions. The first subscript of $\tau$ marks the direction normal to the sheared plane while the second denotes the direction of shear forces. Specimens on Figure 4.2 $\mathbf{a}$ and $\mathbf{b}$ represent the standard shear application parallel to the grain, while shear strength measured on specimens $\mathbf{c}$ and $\mathbf{d}$ are sometimes referred to as rolling shear of solid wood.

Because of the inherent duality of shear stresses, the failure of the specimens may not manifest in the theoretically sheared plane. Furthermore, the unavoidable normal stresses may induce and propagate cracks along the weakest interface within the volume of the specimen. Such out-of-sheared-plane failure may occur with certain grain and ring angle combinations at the earlywood-latewood boundary or along the ray tissues. Consequently, the experimentally determined values can be considered as apparent shear strength only. 


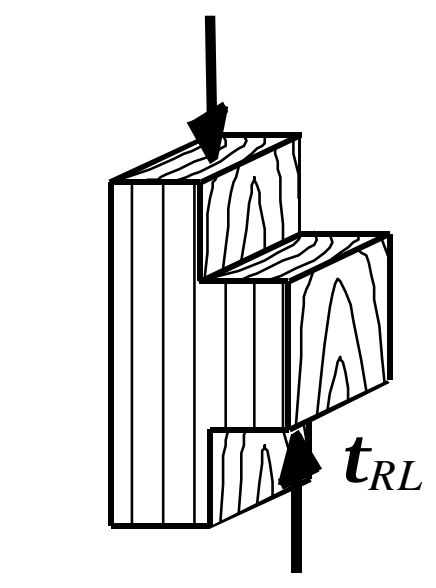

a.
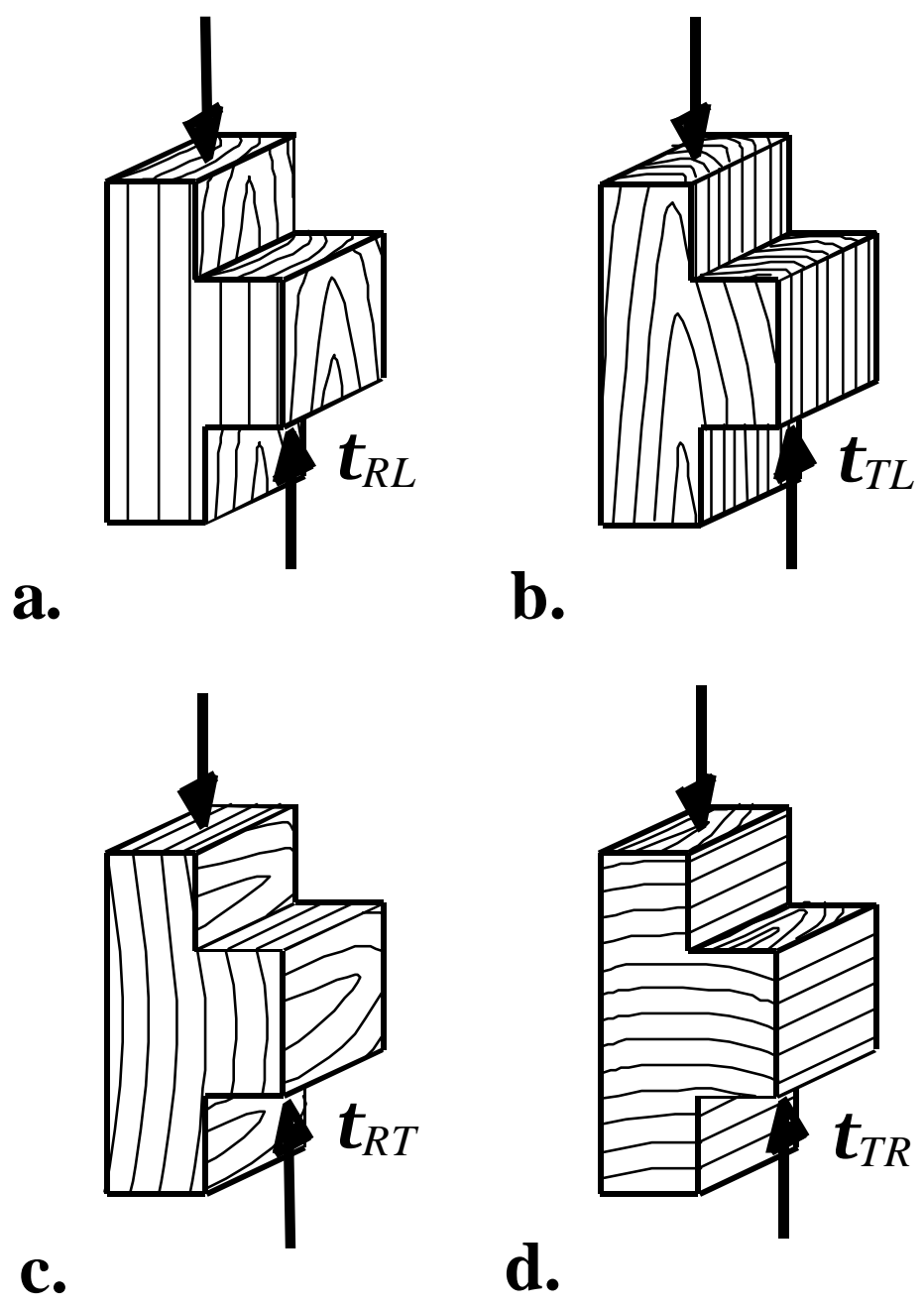

b.

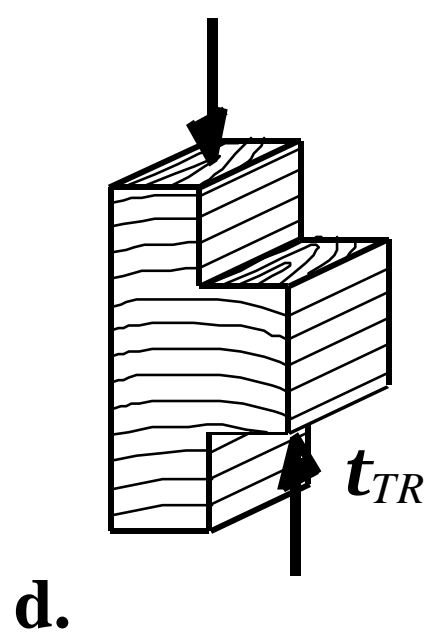

Figure 4.2 - The applied shear forces in the principal anatomical planes and the notation of corresponding shear stresses. $\mathbf{a}, \mathbf{b}$-traditional shear tests, parallel to the grain; $\mathbf{c}, \mathbf{d}$ - rolling shear 


\section{MODELS PREDICTING THE ORTHOTROPY OF SHEAR STRENGTH}

\section{THE ORTHOTROPIC TENSOR THEORY}

In a comprehensive work Szalai (1994) used the orthotropic tensor theory to describe the direction dependent strength and elasticity of wood. Based on Ashkenazi's (1978) strength criteria he applied a four-dimensional tensor approach to predict the shear strength of wood in any oblique plane and direction of shear forces. Substituting the tensor components with the appropriate strength values and eliminating the zero components resulting from the constraint that shear is applied only in the planes parallel to the grain, the equation takes the following form:

$$
\begin{gathered}
\frac{1}{\hat{\tau}_{\varphi}^{\theta}}=\frac{4}{\tau_{90^{\circ}}^{45^{\circ}}} \cos ^{2} \boldsymbol{\theta} \sin ^{2} \theta \sin ^{2} \boldsymbol{\varphi}+\frac{1}{\tau_{R T}} \cos ^{2} 2 \boldsymbol{\theta} \sin ^{2} \boldsymbol{\varphi}+ \\
+\frac{1}{\tau_{T L}} \sin ^{2} \boldsymbol{\theta} \cos ^{2} \boldsymbol{\varphi}+\frac{1}{\tau_{R L}} \cos ^{2} \boldsymbol{\theta} \cos ^{2} \boldsymbol{\varphi}
\end{gathered}
$$

where: $\varphi \quad$ - grain angle;

$\theta \quad$ - ring angle;

$\hat{\tau}_{\varphi}^{\theta} \quad$ - estimated shear strength at grain angle $\varphi$ and ring angle $\theta ;$

$\tau_{i j} \quad$ - shear strength in the main anatomical planes, $(i=R, T ; j=T, L)$ where $i$ is the direction normal of the sheared plane and $j$ is the direction of the applied load;

$\tau_{90^{\circ}}^{45^{\circ}} \quad$ - shear strength at $90^{\circ}$ grain and $45^{\circ}$ ring angle $\left(\varphi=90^{\circ}, \theta=45^{\circ}\right)$.

Note that this solution requires four experimentally predetermined strength values: three obtained in the principal anatomical planes such as $\tau_{R L}, \tau_{T L}$ and $\tau_{R T}$ shown on Figure $4.2 \mathbf{a}, \mathbf{b}$ and $\mathbf{c}$, respectively and a strength value at $90^{\circ}$ grain and $45^{\circ}$ ring angle 
$\left(\tau_{90^{\circ}}^{45^{\circ}}\right)$. The advantages of this model are that it has a firm theoretical basis, uses only four experimentally determined data points for prediction, and is very straightforward.

\section{QUADRATIC MODEL}

Cowin (1979) demonstrated that the shear strength of wood may follow the Hankinson-type strength criterion in a quadratic form. Liu and Floeter (1984) used a tensor polynomial theory, developed by Tsai and $\mathrm{Wu}$ (1971), to re-derive the formula for predicting shear strength in a principal material plane of solid wood. The equation in general form is given as follows:

$$
\hat{\tau}_{\varphi}{ }^{2}=\frac{\tau_{0^{\circ}}{ }^{2} \tau_{90^{\circ}}{ }^{2}}{\tau_{0^{\circ}}{ }^{2} \sin ^{2} \varphi+\tau_{90^{\circ}}{ }^{2} \cos ^{2} \varphi}
$$

where: $\hat{\tau}_{\varphi} \quad$ - estimated shear strength at grain angle $\varphi$;

$\tau_{0^{\circ}} \quad-$ shear strength at grain angle $\varphi=0^{\circ}$;

$\tau_{90^{\circ}} \quad-$ shear strength at grain angle $\varphi=90^{\circ}$.

Like Szalai's approach, this formula has a well-defined theoretical basis. However, it does not include the effect of ring orientation, and has been verified experimentally in the $L T$ plane only, using Sitka spruce specimens.

\section{MODIFIED HANKINSON'S FORMULA}

Kollman (1934) modified the original Hankinson's formula replacing the power 2, to which the trigonometric terms are raised, by an arbitrary power $n$ :

$$
\hat{\tau}_{\varphi}=\frac{\tau_{0^{\circ}} \tau_{90^{\circ}}}{\tau_{0^{\circ}} \sin ^{n} \varphi+\tau_{90^{\circ}} \cos ^{n} \varphi}
$$


The authors claimed that this equation provides better fit than the original Hankinson's formula for predicting tensile strength and modulus of elasticity. Although this model is purely empirical, it has a capability to describe peak shear stresses at inclined grain, by using a higher power (i.e., $n>2$ ). Beside the lack of theoretical basis, this model is probably very species specific and requires a significant database for accurate determination of the value of $n$. Like the quadratic formula, it can handle only fixed ring orientation in its present form.

\section{COMBINED MODELS}

So far, the orthotropic tensor theory was the only model that could handle both grain and ring angle changes. Researchers addressed the effect of ring orientation on the shear strength parallel to the grain and usually found it negligible. The apparent low degree of orthotropy of shear strength between the $L T$ and $L R$ main anatomical planes (i.e., $\tau_{R L} \approx \tau_{T L}$ ) did not trigger extensive model development to describe the phenomenon. The only available model was published by Szalai (1994). It includes two equations derived from tensor analysis, as follows:

$$
\begin{gathered}
\hat{\tau}_{0^{\circ}}^{\theta}=\frac{1}{\left(\cos ^{2} \theta / \tau_{R L}+\sin ^{2} \theta / \tau_{T L}\right)} \\
\hat{\tau}_{90^{\circ}}^{\theta}=\frac{1}{\cos ^{4} \theta / \tau_{R T}+\sin ^{4} \theta / \tau_{T R}+\left(1 / \tau_{90^{\circ}}^{45^{\circ}}-1 / 4 \tau_{R T}-1 / 4 \tau_{T R}\right) \sin ^{2} 2 \theta}
\end{gathered}
$$


where: $\hat{\tau}_{0^{\circ}}^{\theta} \quad$ - estimated shear strength at $\theta$ ring angle, $\varphi=0^{\circ}$;

$\hat{\tau}_{90^{\circ}}^{\theta} \quad$ - estimated shear strength at $\theta$ ring angle, $\varphi=90^{\circ}$;

and the other symbols are as given at Equation 4.1.

Equation 4.4 approximates the shear strength of traditional, parallel to the grain specimens as a function of ring orientation. It requires two experimentally predetermined strength values. The rolling shear strength variations are given by Equation 4.5 where three predetermined strength values are needed. Note that $\tau_{R T}$ and $\tau_{T R}$ represent the maximum stresses (i.e., shear strength) values. Due to the duality, the stresses in these two directions are identical. However, this is not necessarily true for the strength values of wood because of the unpredictable failure mode, as discussed earlier. Although these equations have not been experimentally verified, theoretically they should describe the effect of ring orientation on the shear strength of orthotropic materials.

One can realize that these equations can provide predetermined strength data for the quadratic model and for the modified Hankinson's formula for predicting the effect of grain orientation. Consequently, combining Equations 4.4 and 4.5 with Equations 4.2 or 4.3, we can obtain additional two models for estimating the orthotropy of shear strength as a function of grain and ring orientation. This combination for the quadratic model is given in a short-hand form as follows:

$$
\hat{\tau}_{\varphi}^{\theta^{2}}=\frac{\hat{\tau}_{0^{\circ}}^{{ }^{2}} \hat{\tau}_{90^{\circ}}{ }^{2}}{\hat{\tau}_{0^{\circ}}^{\theta^{2}} \sin ^{2} \varphi+\hat{\tau}_{90^{\circ}}^{{ }^{2}} \cos ^{2} \varphi}
$$


Furthermore, using the modified Hankinson's formula we obtain:

$$
\hat{\tau}_{\varphi}^{\theta}=\frac{\hat{\tau}_{0^{\circ}}^{\theta} \hat{\tau}_{90^{\circ}}^{\theta}}{\hat{\tau}_{0^{\circ}}^{\theta} \sin ^{n} \varphi+\hat{\tau}_{90^{\circ}}^{\theta} \cos ^{n} \varphi}
$$

Figure 4.3 gives a graphical explanation of these combined models. Note that both of these approximations require five experimentally predetermined strength values and Equations 4.6 or 4.7 should be solved $m$ times where $m$ is the resolution, calculated as $m=(1+90 /$ ring angle increment $)$. During this research these two models along with the orthotropic tensor theory (Equation 4.1) were fitted to experimental data and statistically analyzed.

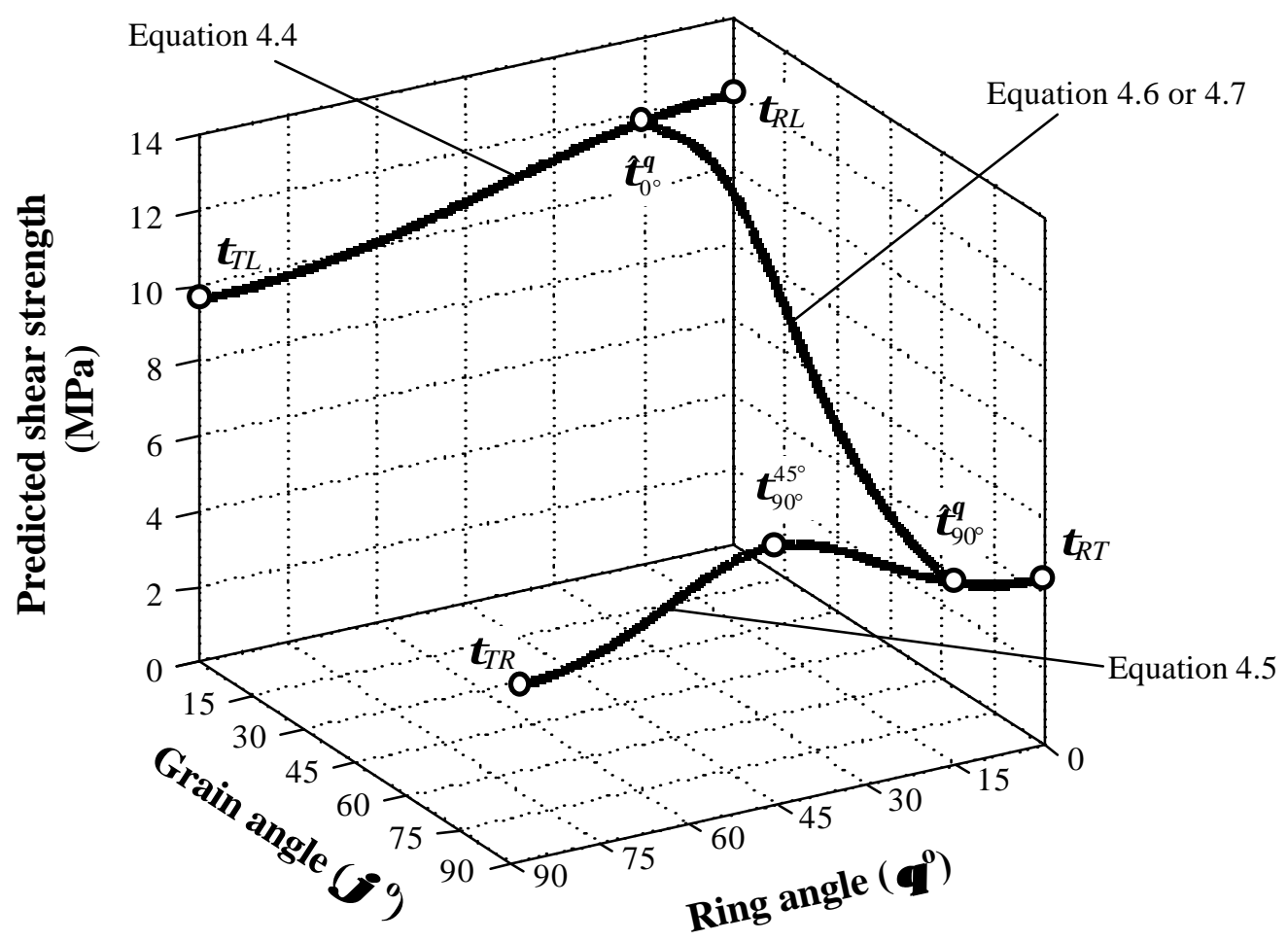

Figure 4.3 - Interpretation and the principle of prediction process of the combined models 


\subsubsection{Orthotropy of compression strength and elasticity}

\subsubsection{Shear stresses in compression specimens}

Although wood is usually modeled as an orthotropic material, in reality it is cylindrically orthotropic. The inherent natural variability of wood coupled with the ambiguous definition of failure stresses in compression generates several difficulties in application of advanced failure theories. Furthermore, the compression strength determination of cross-grained wood blocks always depends on the size, shape, manufacturing precision (parallelism) and support conditions of the specimen.

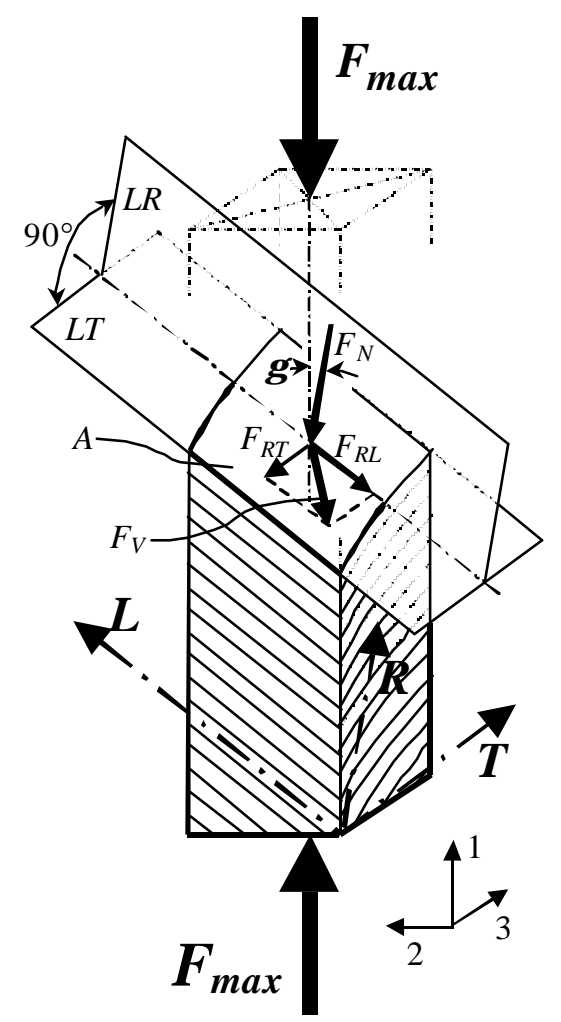

Figure 4.4 - Internal force conditions of an oblique specimen under compression 
Consider an ideal, cross-grained specimen for compression strength assessment in a global coordinate system, as shown in Figure 4.4, where the anatomical directions ( $L, R$ and $T$ ) are marked, as well. In an arbitrarily selected $L T$ principal anatomical plane of the specimen, the compression force $\left(F_{\max }\right)$ can be broken into a normal $\left(F_{N}\right)$ and an in-plane $\left(F_{V}\right)$ component. The area $(A)$ of this inclined sectional surface can be approximated using trigonometric identities. $F_{V}$ can be represented as a resultant of $F_{R L}$ and $F_{R T}$ that induce traditional parallel to the grain shear stress and rolling shear, respectively, at $0^{\circ}$ ring angle. If the ratio of $F_{V}$ to the sectional area of the specimen $\left(F_{V} / A\right)$ in the $L T$ plane exceeds the shear strength $\left(\tau_{\vartheta}^{\ominus}\right)$ the specimen will fail in shear under compression. The subscripts, $\vartheta$ and $\Theta$ are the angles between $F_{V}$ and the direction of the fibers, and between the sheared and $L T$ planes, respectively. For this particular case $\Theta=0$. Because several species have relatively low degree of shear orthotropy concerning the ring orientation, one can realize that shear failure is possible in any plane between $L T$ and $L R$, if the induced shear stresses are large enough. In fact, off axis compression specimens have been used to evaluate the shear strength of Finnish pine in the $L T$ and $L R$ planes by Ylinen (1963). The relationship between shear and normal stresses for grain inclination range of $12^{\circ}<\varphi<32^{\circ}$ was given as follows:

$$
\tau_{\max }=1 / 2 \sigma_{\max } \sin 2 \varphi
$$

One should note that this shear failure stress is biased by a compression stress $\left(\sigma_{q}\right)$ normal to the plane of failure:

$$
\sigma_{q}=\sigma_{\text {nax }} \sin ^{2} \varphi,
$$


where $\sigma_{\max }=\sigma_{l}$ the ultimate compression stress in the global coordinate system and $\varphi$ is the grain angle.

Experimental results of Ylinen (1963) showed that there is a consistent shear strength difference at the studied grain angles between the $L R$ and $L T$ planes. This indicates some interactions between grain and ring orientations. The ambiguous failure modes, coupled with the inherent natural variability of wood, allow the exploration of apparent strength only for particularly defined specimens. The size, the slenderness ratio of the specimen and the end conditions along with the existence or lack of lateral supports may affect the measured compression strength values. Furthermore, the determination of MOE in compression may be also biased by some of these conditions and the induced shear strain may affect the measured normal strain.

The complexity of the failure phenomena, and the above-discussed problems make the predictions difficult and sometimes unreliable. However, some approaches that incorporate both the ring and grain angle variations can predict the orthotropic properties with reasonable accuracy. Such methods, that were verified and used during this research, are briefly discussed next.

\subsubsection{Models describing the orthotropic compression properties}

Selection criteria for models that can predict both MOE and strength were simplicity, minimum input requirements and validity at any grain $(\varphi)$ and ring angle $(\theta)$ combinations. (The definition of $\varphi$ and $\theta$ for purposes of compression strength and MOE determination is illustrated on Figure 4.5.) While the simplicity is not a vital problem because of the advances in computer science, models that needed all nine independent 


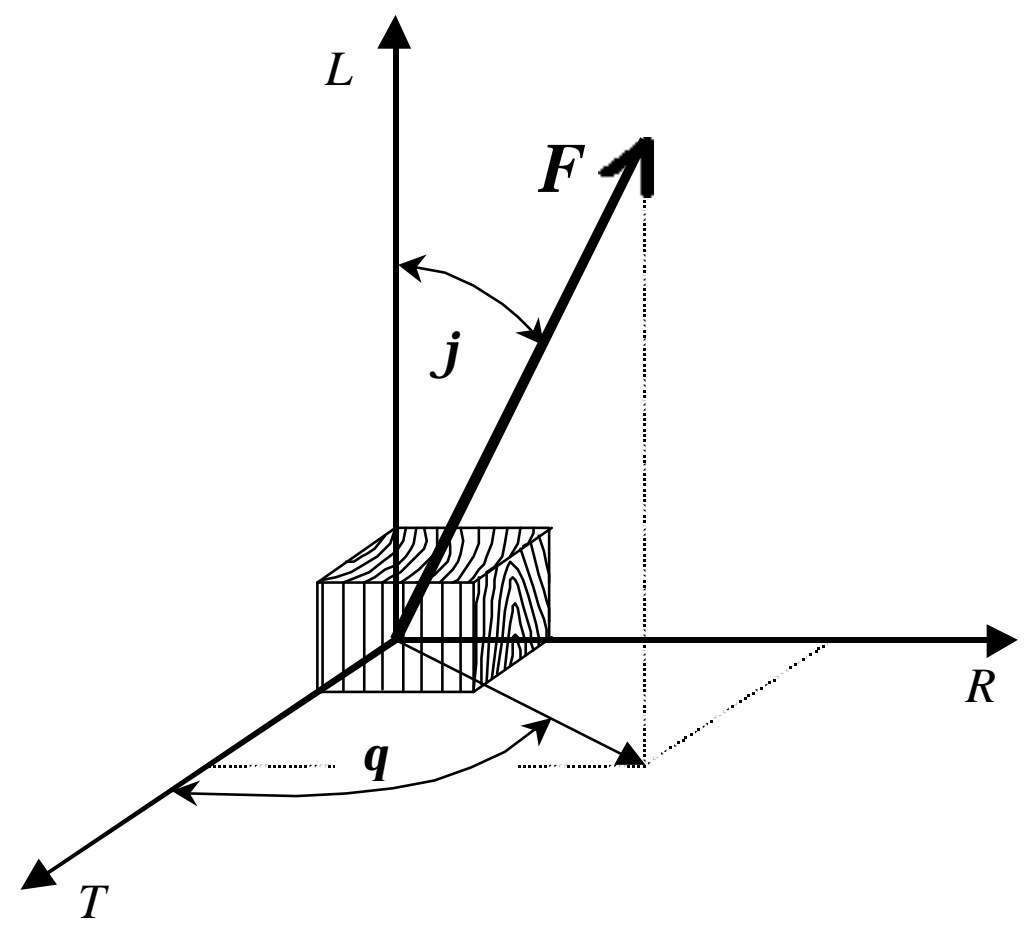

Figure 4.5 - Interpretation of grain angle $(\varphi)$ and ring orientation $(\theta)$ of the applied compression load

elastic constants as inputs were not considered. Therefore, two procedures, a theoretical and an empirical, were selected.

\section{STRENGTH /STIFFNESS TENSOR THBORY}

Szalai (1994) provided an approach for calculating the normal strength of orthotropic materials in any direction. The equation can be derived from the fourdimensional strength tensor by transforming the first element of the tensor, as described in APPENDIX A. After applying various strength criteria to the transformed element and eliminating the zero components, the equation takes the following form: 


$$
\begin{aligned}
\frac{1}{\hat{\sigma}_{\varphi}^{\theta}}= & \frac{1}{\sigma_{L}} \cos ^{4} \varphi+\frac{1}{\sigma_{R}} \sin ^{4} \varphi \sin ^{4} \theta+\frac{1}{\sigma_{T}} \sin ^{4} \varphi \cos ^{4} \boldsymbol{\theta} \\
& +\left(\frac{4}{\sigma_{90^{\circ}}^{45^{\circ}}}-\frac{1}{\sigma_{R}}-\frac{1}{\sigma_{T}}\right) \sin ^{4} \varphi \sin ^{2} \theta \cos ^{2} \boldsymbol{\theta} \\
& +\left(\frac{4}{\sigma_{45^{\circ}}^{0^{\circ}}}-\frac{1}{\sigma_{L}}-\frac{1}{\sigma_{T}}\right) \cos ^{2} \varphi \sin ^{2} \varphi \cos ^{2} \boldsymbol{\theta} \\
& +\left(\frac{4}{\sigma_{45^{\circ}}^{90^{\circ}}}-\frac{1}{\sigma_{L}}-\frac{1}{\sigma_{R}}\right) \cos ^{2} \varphi \sin ^{2} \varphi \sin ^{2} \boldsymbol{\theta}
\end{aligned}
$$

where: $\hat{\sigma}_{\varphi}^{\theta} \quad$ - predicted compression strength at grain angle $\varphi$ and ring angle $\boldsymbol{\theta}$

$\sigma_{i} \quad$ - compression strength in the principal anatomical directions $(i=L, R, T)$;

$\sigma_{i}^{j} \quad$ - compression strength at $\varphi=i ; \theta=j$.

The six strength values used in Equation 4.10 should be determined experimentally. Note that replacing the strength with the appropriate $E_{i}$ or $E_{i}^{j}$ values, the evaluation of orthotropic elasticity can be performed.

\section{THREE-DIMENSIONAL HANKINSON ‘S FORMULA}

Bodig and Jayne (1982) based their approach partly on the Hankinson formula, and on the observed compression strength pattern in the $R T$ plane. According to the authors, the strength variation pattern in this plane consists of a linear and a sinusoidal component:

$$
\hat{\boldsymbol{\sigma}}_{90^{\circ}}^{\theta}=\left[\sigma_{T}+\frac{\theta}{\pi / 2}\left(\sigma_{R}-\sigma_{T}\right)\right]+\left[K(-\sin 2 \theta) \frac{\sigma_{R}+\sigma_{T}}{2}\right],
$$


where: $\hat{\boldsymbol{\sigma}}_{90^{\circ}}^{\theta} \quad-$ the predicted compression strength at $90^{\circ}$ grain angle and ring angle $\boldsymbol{\theta}$; $K \quad$ - empirical constant ( 0.2 for hardwoods).

Other notations are as in Equation 4.10.

After calculating this value for a certain ring angle, strength or MOE properties belonging to any grain angle at the given ring angle level can be obtained by substituting this value and $\sigma_{L}$ into the Hankinson formula:

$$
\hat{\sigma}_{\varphi}^{\theta}=\frac{\sigma_{L} \hat{\sigma}_{90^{\circ}}^{\theta}}{\sigma_{L} \sin ^{2} \varphi+\hat{\sigma}_{90^{\circ}}^{\theta} \cos ^{2} \varphi}
$$

This empirical approach lacks the firm theoretical basis of the orthotropic tensor theory. In contrast, this method requires only three experimentally determined data points in the principal anatomical directions, and the calculation is no more difficult than the one used in the previous formula.

\subsubsection{Orthotropy of the dynamic elastic parameters}

Section 4.1.2.2 proposes two simple and effective models that describe compression strength and elasticity as a function of grain and ring angle. The same models pertain to the dynamic MOE of wood. In the present study, dynamic MOE was assessed as a function of grain angle $(\varphi)$ only, in the $L T$ plane. The two models presented in the previous section apply to this situation, as follows: 


\section{ORTHOTROPIC TENSOR THEORY}

Equation 4.10 presents the orthotropic solution for predicting compression or tensile strength and elasticity if both grain and ring angle are variable. For predicting the MOE in the $L T$ plane only (i.e., $\theta=0^{\circ}$ ), the formula reduces to the following:

$$
\hat{E}_{\varphi}=\frac{1}{\frac{1}{E_{L}} \cos ^{4} \varphi+\frac{1}{E_{T}} \sin ^{4} \varphi+\left(\frac{4}{E_{45^{\circ}}^{0^{\circ}}}-\frac{1}{E_{L}}-\frac{1}{E_{T}}\right) \sin ^{2} \varphi \cos ^{2} \varphi},
$$

where: $\hat{E}_{\varphi} \quad-$ predicted MOE at grain angle $\varphi$;

$E_{L}, E_{T}$ - experimentally determined MOE in the longitudinal and tangential directions;

$E_{45^{\circ}}^{0^{\circ}} \quad-$ experimentally determined MOE in the $L T$ plane, at $\varphi=45^{\circ}$.

\section{HANKINSON's FORMULA}

For MOE in the LT plane, Hankinson's Formula (1921) applies in the following form:

$$
\hat{E}_{\varphi}=\frac{E_{L} E_{T}}{E_{L} \sin ^{2} \varphi+E_{T} \cos ^{2} \varphi},
$$

The advantages and disadvantages of the above two theories are the same as described in section 4.1.2.2, including the fact that, in this case, Hankinson's formula uses one less value for prediction. 


\subsection{Prediction of MOE by stress-wave propagation}

The propagation velocity of longitudinal stress-waves in a material is directly related to the modulus of elasticity and the bulk density of the substance:

$$
E_{D}=v^{2} \rho
$$

where: $E_{D} \quad$ - dynamic Modulus of Elasticity ( $\mathrm{Pa}$ );

$v \quad-$ propagation velocity $(\mathrm{m} / \mathrm{s})$;

$\rho \quad-$ density $\left(\mathrm{kg} / \mathrm{m}^{3}\right)$.

The above equation holds true for so-called one-dimensional bodies only; that is, where the dimensions perpendicular to the wave propagation are at least one magnitude smaller than the wave length. In veneer sheets, where one cross-sectional dimension is larger than the wave length (two-dimensional body), the above equation is modified with the Poisson ratio:

$$
E_{D}=v^{2} \rho\left(1-v_{i j}^{2}\right)
$$

where: $v_{i j} \quad$ - Poisson ratio ( $i$ - propagation direction; $j$ - perpendicular in-plane direction.)

Unfortunately, reliable information concerning $v_{i j}$ is seldom available. In past investigations, stress-wave MOE measured on veneer was invariably calculated using Equation 4.15. The results of Jung's investigations (1979) indicate that the correction introduced in Equation 4.16 might be negligible in wood veneers.

Because of the viscoelastic nature of wood, measured dynamic MOE depends on the rate of stress-development. During dynamic testing, stresses develop much faster than they do during static testing, and the difference between the resulting MOE values is 
significant. For this reason, MOE calculated from longitudinal or transverse vibration characteristics is called dynamic MOE. Divos and Tanaka (2000) reported the following empirical equation to calculate the ratio of dynamic and static MOE:

$$
\frac{E_{D}}{E_{S}}=1+0.017 \log \left(\frac{t_{S}}{t_{D}}\right)
$$

where: $E_{D}, E_{S}-$ dynamic and static MOE, respectively

$t_{D}, t_{S} \quad$ - characteristic time of the dynamic and static MOE determination, respectively.

\subsection{Composite simulation}

Although the structure of the two composite types involved in this study is largely different, the basic principles used in their simulation are the same. The basis for simulating the bending elasticity of composites is the so-called laminate theory (Bodig and Jayne 1982):

$$
E=\frac{\sum E_{i} I_{i}}{I}
$$

where: $E, I-$ MOE and $2^{\text {nd }}$ order moment of inertia of the cross-section, respectively;

$E_{i}, I_{i}-$ MOE and moment of inertia of the $i^{\text {th }}$ layer, with respect to the composite's neutral axis, respectively.

This theory applies directly to layered composites like LVL. It is possible to adapt this concept to non-layered systems, such as PSL, as well. In this case, the above summation involves individual strands, rather than layers. 
The simulation of Compression MOE is based on the calculation of the external work applied to a body and the internal energy stored therein. These quantities are equal in the linear elastic region. $\mathrm{Xu}$ and Suchsland (1998b) derived the following equation from this equality:

$$
E=\frac{\Sigma E_{i} V_{i}}{V}
$$

where: $E, V$ - MOE and total volume of the composite;

$E_{i}, V_{i}$ - MOE and volume of the $i^{\text {th }}$ constituent. 


\section{Materials AND MethodS}

\subsection{Orthotropic strength and elasticity}

\subsubsection{Raw materials}

Orthotropic strength and elasticity determination of solid wood involved three Appalachian hardwood species. These species are either already used in composite manufacture, or are potential raw materials of structural composites.

1. Quaking aspen (Populus tremuloides Michx.) is a light colored, light, soft, fastgrowing wood species. It is produced primarily in the Northeastern and Lake States, with some production in the Rocky Mountain States. (Wood Handbook 1999). Aspen is generally considered as a low-value wood, with low strength and elastic properties. Aspen material is cheap, and has excellent potential as a raw-material for value-added products, such as composite lumber.

2. Red oak (Quercus rubra L.) is a heavy, hard, dark-colored wood species that is prevalent in the Eastern United States. It is used primarily as sawn lumber for furniture and cabinetry, flooring, moulding and millwork, and as decorative veneer. The mechanical properties of this species are typically good. Although red oak is harder to convert into veneer, it has good potential as composite raw-material, because of its ready availability and excellent mechanical parameters.

3. Yellow-poplar (Liriodendron tulipifera L.) is a moderately light and soft, light colored hardwood. It is used mostly for furniture, musical instruments, structural components and raw material for plywood manufacture. This wood species is moderately weak mechanically. It is currently used for producing LVL and PSL structural lumber in the Eastern U.S. that has a plentiful resource of yellow-poplar. 


\subsubsection{Specimen preparation; physical properties}

Trees from the above species were harvested from the West Virginia University forest, located in Monongalia and Preston counties, and sawn into lumber, or peeled into $3.2 \mathrm{~mm}(1 / 8 \mathrm{in})$ thick structural veneer. Prior to testing, specimens were conditioned to approximately $12 \%$ moisture content in a controlled environment (i.e., $21^{\circ} \mathrm{C}$ and $65 \%$ $\mathrm{RH})$. Representative samples of specimens were prepared and tested for moisture content and specific gravity, according to ASTM D 4442-92, method A and ASTM D 2395-93, method A, respectively.

\subsubsection{Orthotropy of shear strength}

Figure 5.1 shows the specimen shape and target dimensions which differed from that specified by the ASTM D 143-94 standard. The double-notched shear blocks were prepared from blanks having varying ring and grain angle between $0^{\circ}$ and $90^{\circ}$ with $15^{\circ}$ increments. Figure 5.2 demonstrates this specimen preparation practice. Test series included sets for all combinations of the above angles, for all the examined species. The sample size for these sets varied between six and fifteen. Shear forces were applied through a special device providing a single plane of shear within the specimens. The area of the sheared section was approximately $500 \mathrm{~mm}^{2}$ according to the target dimensions shown on Figure 5.1. Lang (1997) discussed the advantages of this alternative shear strength assessment and the description of the device in details. Figure $\mathbf{5 . 3}$ shows the principal and schematic of the shear testing apparatus along with the experimental setup. The justification of this alternative testing method lies in the smaller specimen 


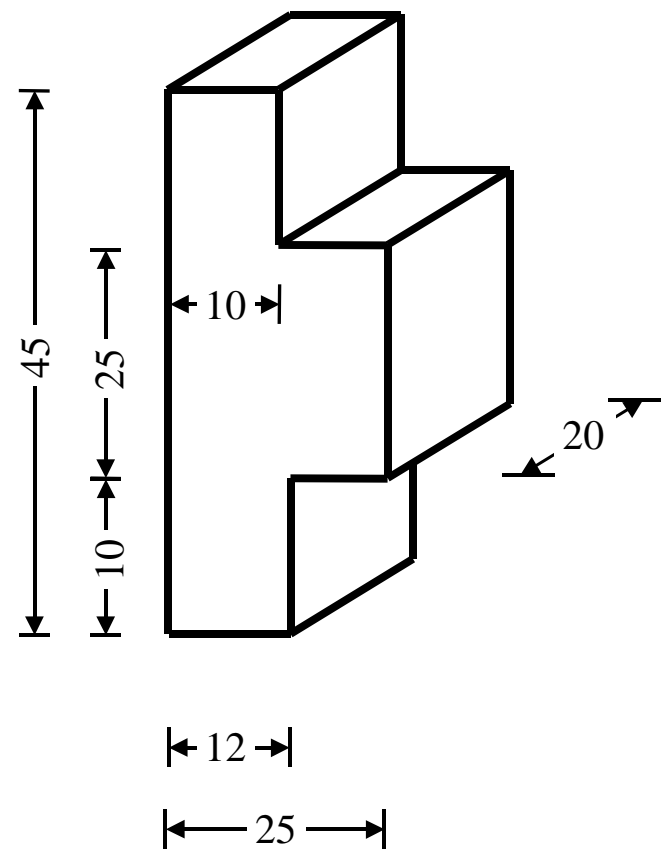

Figure 5.1 - Dimensions of the double-notched shear specimens

dimensions for which the grain and ring orientations are better controlled. Furthermore, it requires significantly less volume of raw material and waste is minimized when machining more than 1200 specimens.

Tests were conducted using an MTS universal servo-hydraulic testing equipment mounted with $100 \mathrm{kN} \pm 1 \mathrm{~N}$ load cell. The machine operated under displacement control with a rate of speed of $0.6 \mathrm{~mm} / \mathrm{min}$ required by the ASTM D 143 - 94 standard. 

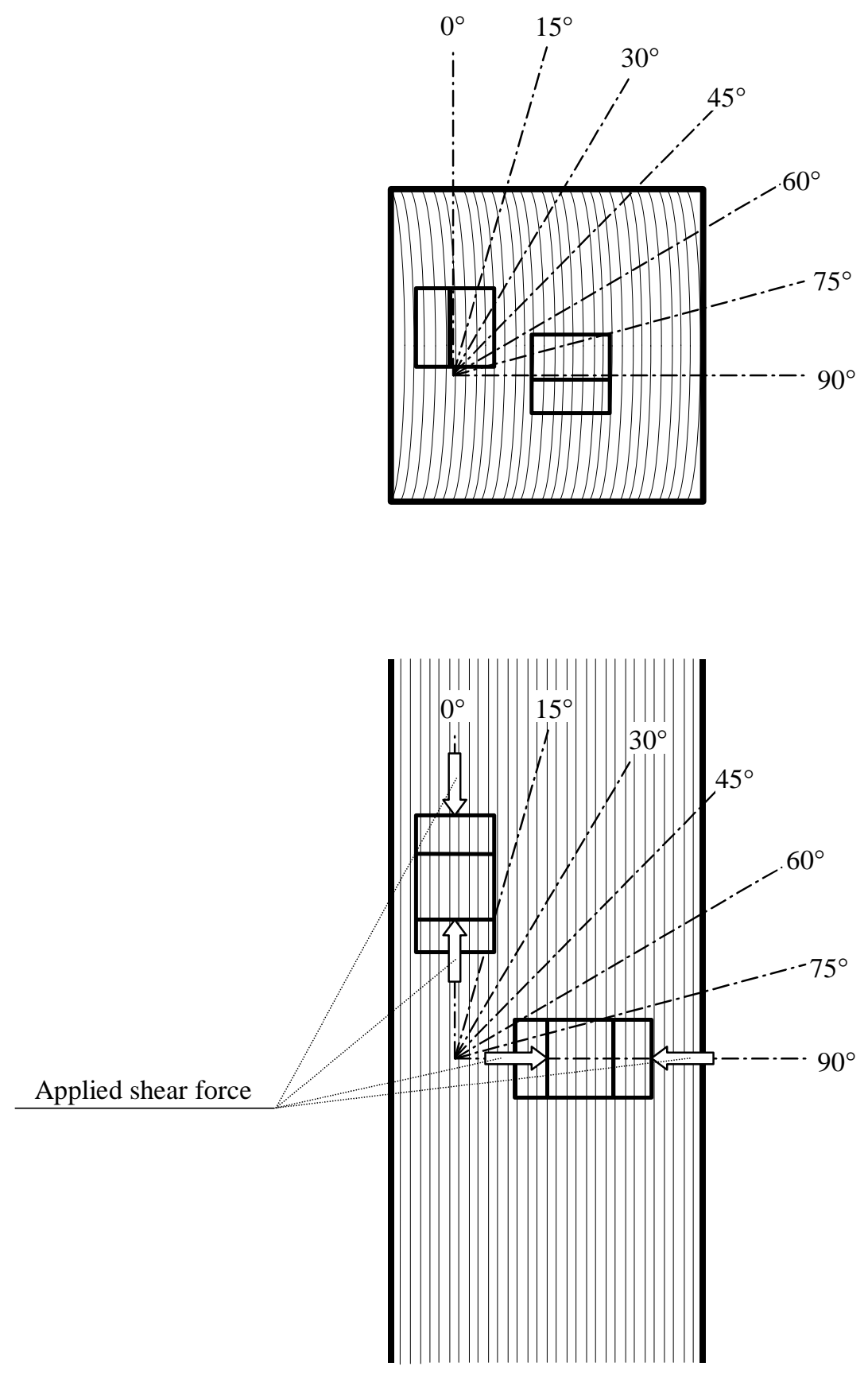

Figure 5.2 - Schematic of the specimen manufacturing practice from prepared, straight-grained blanks 

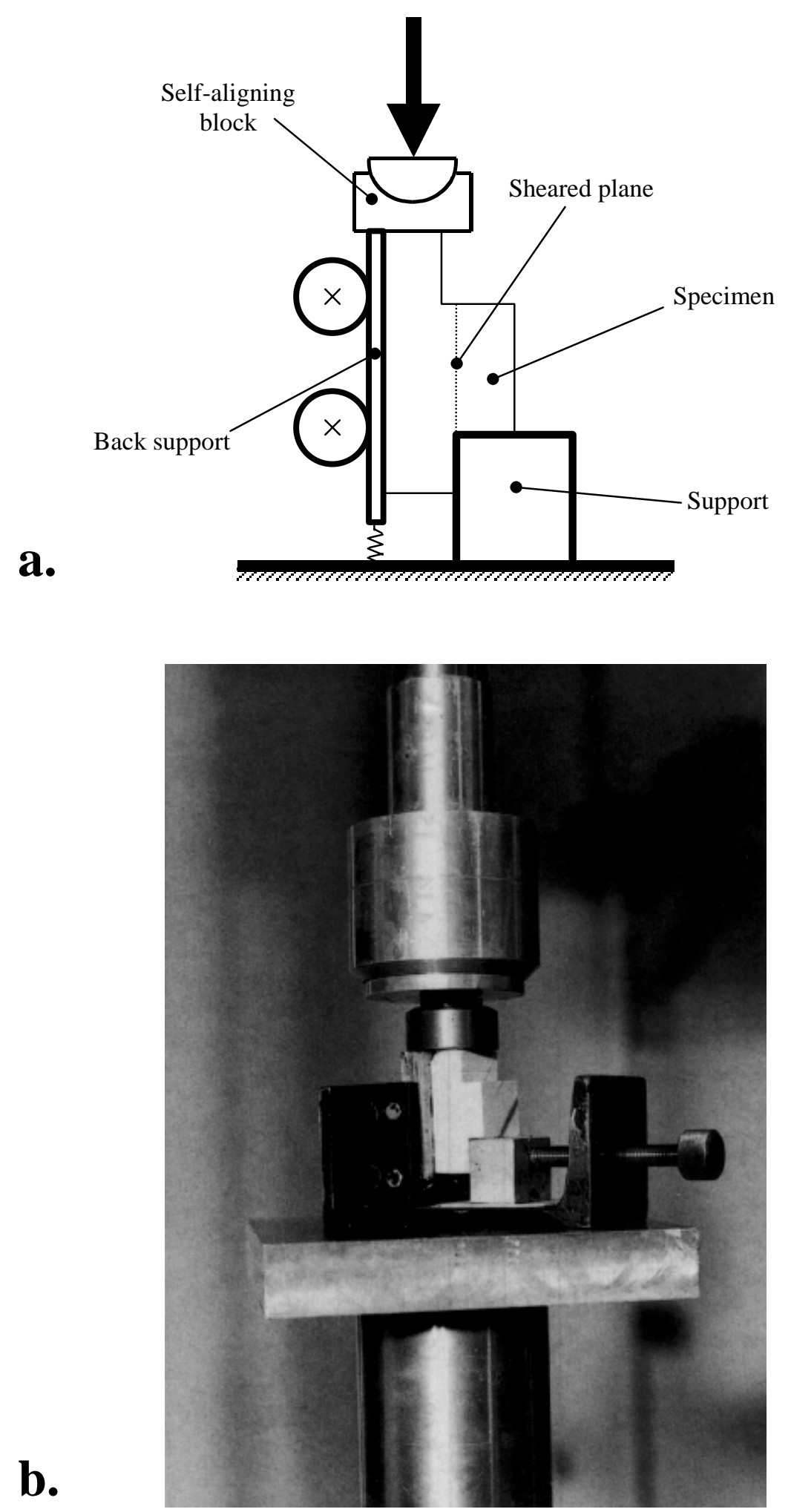

Figure 5.3 - Schematic of the shear testing apparatus and the experimental setup 


\subsubsection{Orthotropy of compression strength and elasticity}

Standard ASTM specimens (ASTM D 143 - 94, secondary method) were prepared from each species. The final dimensions $(25 \times 25 \times 100 \mathrm{~mm})$ were set after conditioning the blank materials.

Specimen groups for compression strength and MOE determination were laid out similar to shear strength specimens. Ring and grain angles of the specimens varied between $0^{\circ}$ and $90^{\circ}$ with $15^{\circ}$ increments. Ring angle is not defined at $0^{\circ}$ grain orientation and its effect was disregarded at $15^{\circ}$ grain angle. The experimental design, therefore, contained only one set of 10 specimens at both $0^{\circ}$ and $15^{\circ}$. Figure 5.4 explains the specimen manufacturing practice.

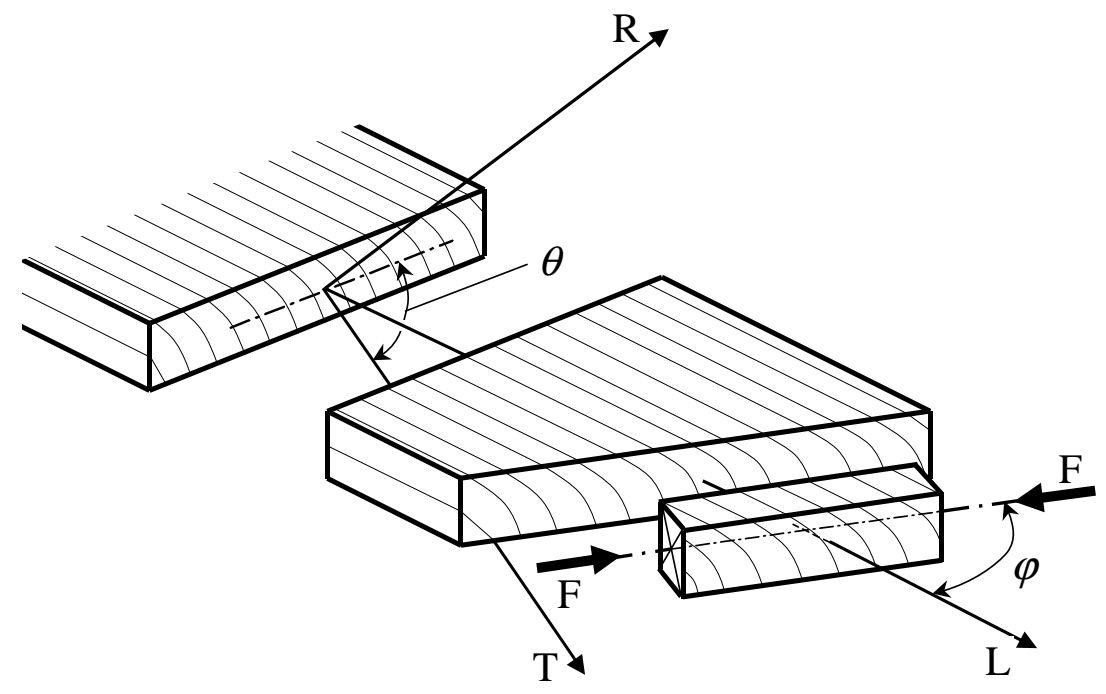

Figure 5.4 - Compression specimen manufacturing practice and the interpretation of $\varphi$ and $\theta$ 

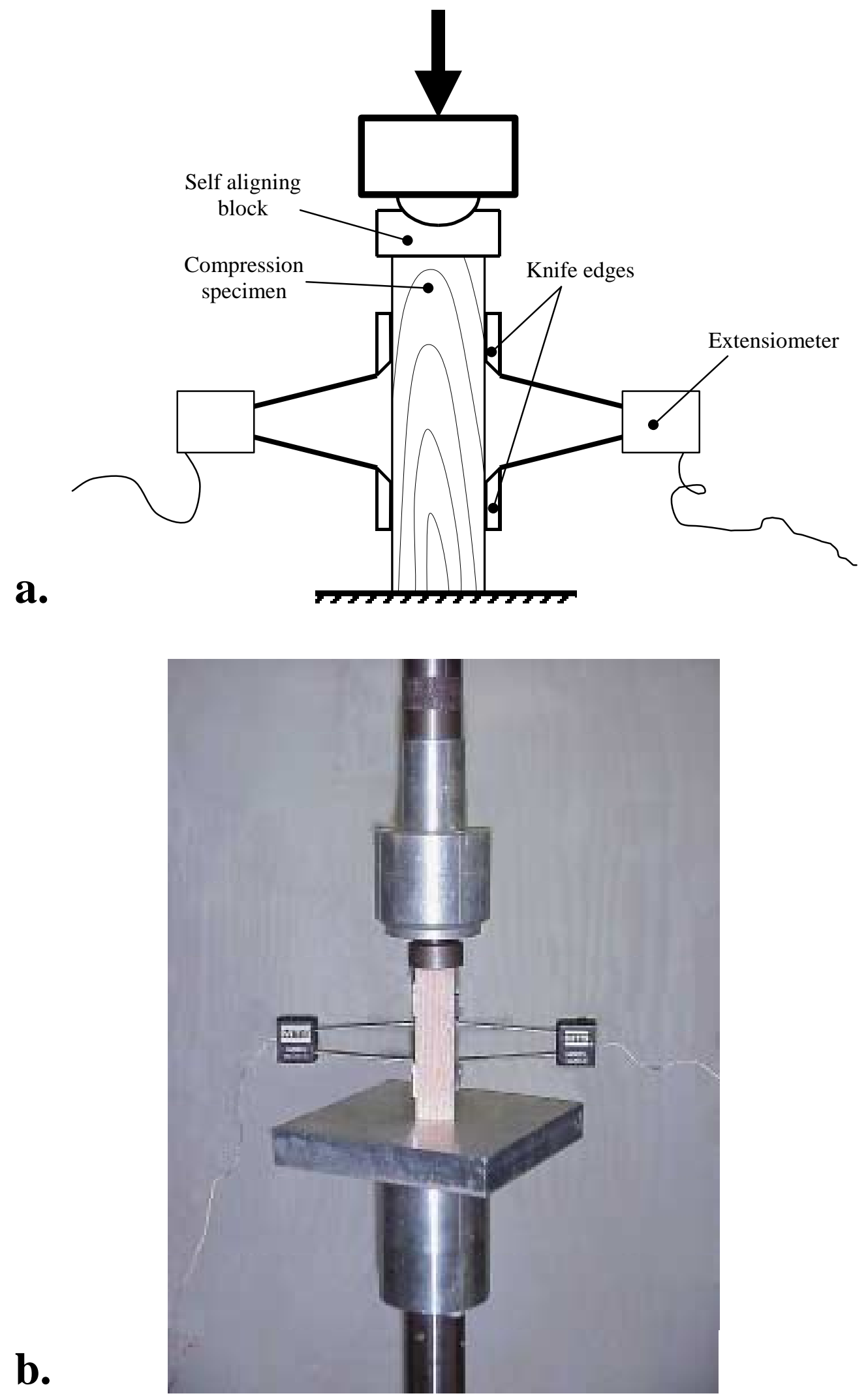

Figure 5.5 - Compression force application and the two-sided strain measurement 
Approximately 160 specimens by species provided 4 to 10 replications at the investigated variable $(\varphi, \theta)$ combination. The testing machine was the same as used for shear tests. Cross-head movement was under displacement control. Compression load application happened through a self-aligning block placed on top of the specimen. A computerized data acquisition system collected the load and displacement data in real time. Screw-mounted knife-edge pieces held in place the clip-on extensiometers that provided displacement data throughout the test, over a gauge length of $41 \mathrm{~mm}$. Two gauges measured the deformations at the opposite, side-grained surfaces of the specimens. The obtained data pairs were averaged and the corresponding loaddisplacement values were converted into stress-strain diagrams for further analyses. Figure 5.5 shows the experimental assembly. Other parameters of the procedure, including the speed of testing, agreed with the specifications of ASTM D 143 - 94 standard. Load application continued until failure or until the densification plateau of horizontally grained $\left(\varphi \approx 90^{\circ}\right)$ specimens were explicitly reached. The failure type of each specimen was visually assessed and recorded.

\subsubsection{Orthotropy of tensile elasticity}

Experimental material consisted of $3.2 \mathrm{~mm}$ (1/8 in) thick structural veneer sheets made of the three Appalachian hardwood species. The sheets were peeled and dried in a commercial structural composite plant.

The equipment used for dynamic MOE determination was an ultrasonic device, developed by Hungarian researchers (Divos, 2000). The device consists of an ultrasonic timer and two piezoelectric accelerometers. The transducers use a $127 \mathrm{~V}, 45 \mathrm{kHz}$ impulse 

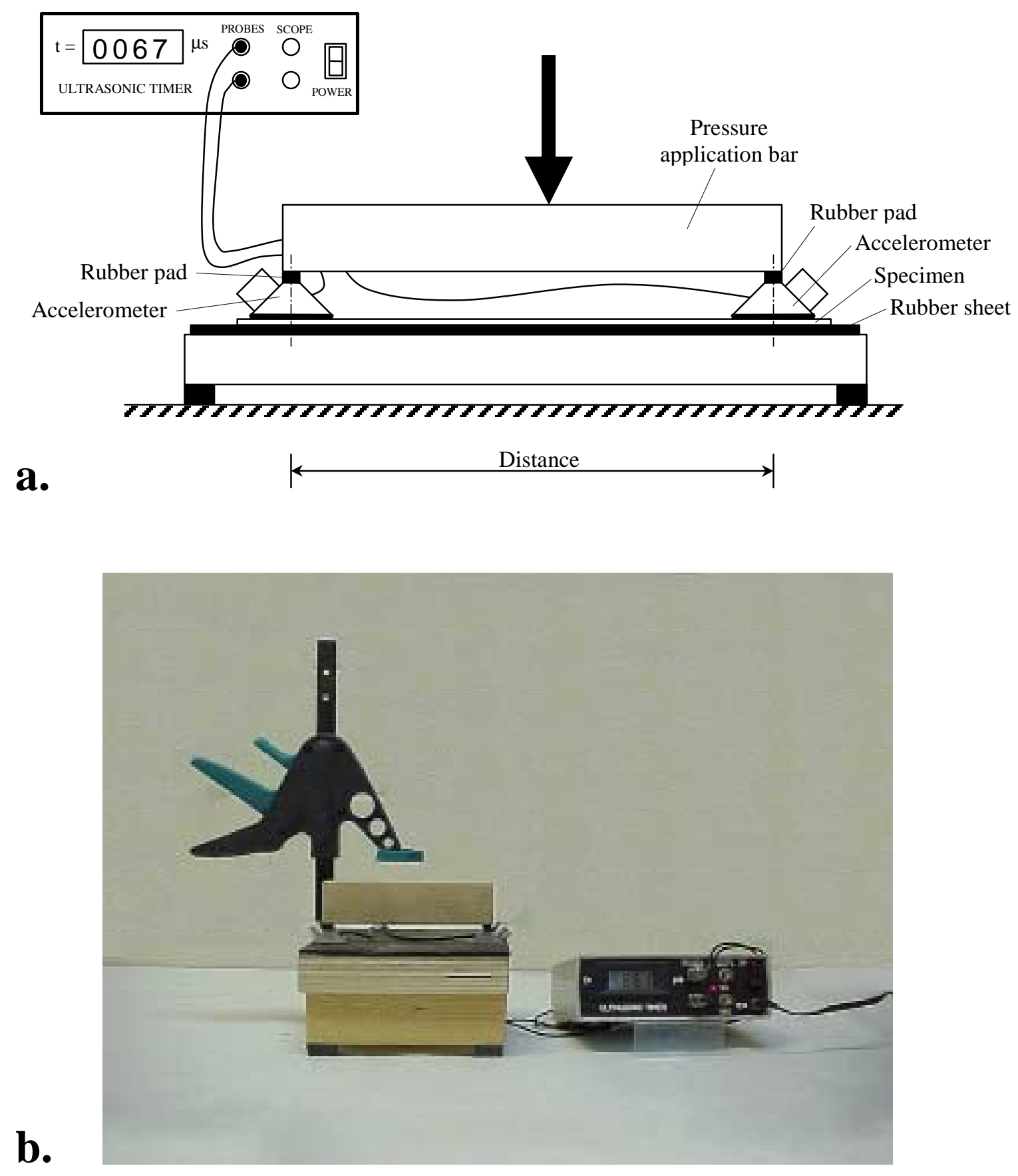

Figure 5.6 - Schematic of the ultrasonic testing equipment and the experimental setup 
that lasts for $30 \mu \mathrm{s}$. The impulses follow at 1s intervals. 3-4 MPa surface pressure between the transducers and the veneer sheets provides adequate contact, using sandpaper as coupling material.

Figure 5.6 shows the schematic of the ultrasonic equipment and a picture of the experimental setup. The distance between the transducer and the receiver (i.e. measurement span) was $160 \mathrm{~mm}$. The material was clamped on a special table that was covered with a rubber sheet to avoid bridging of the signal between the transducer and the receiver. A clamp provided adequate surface pressure between the transducers and the material.

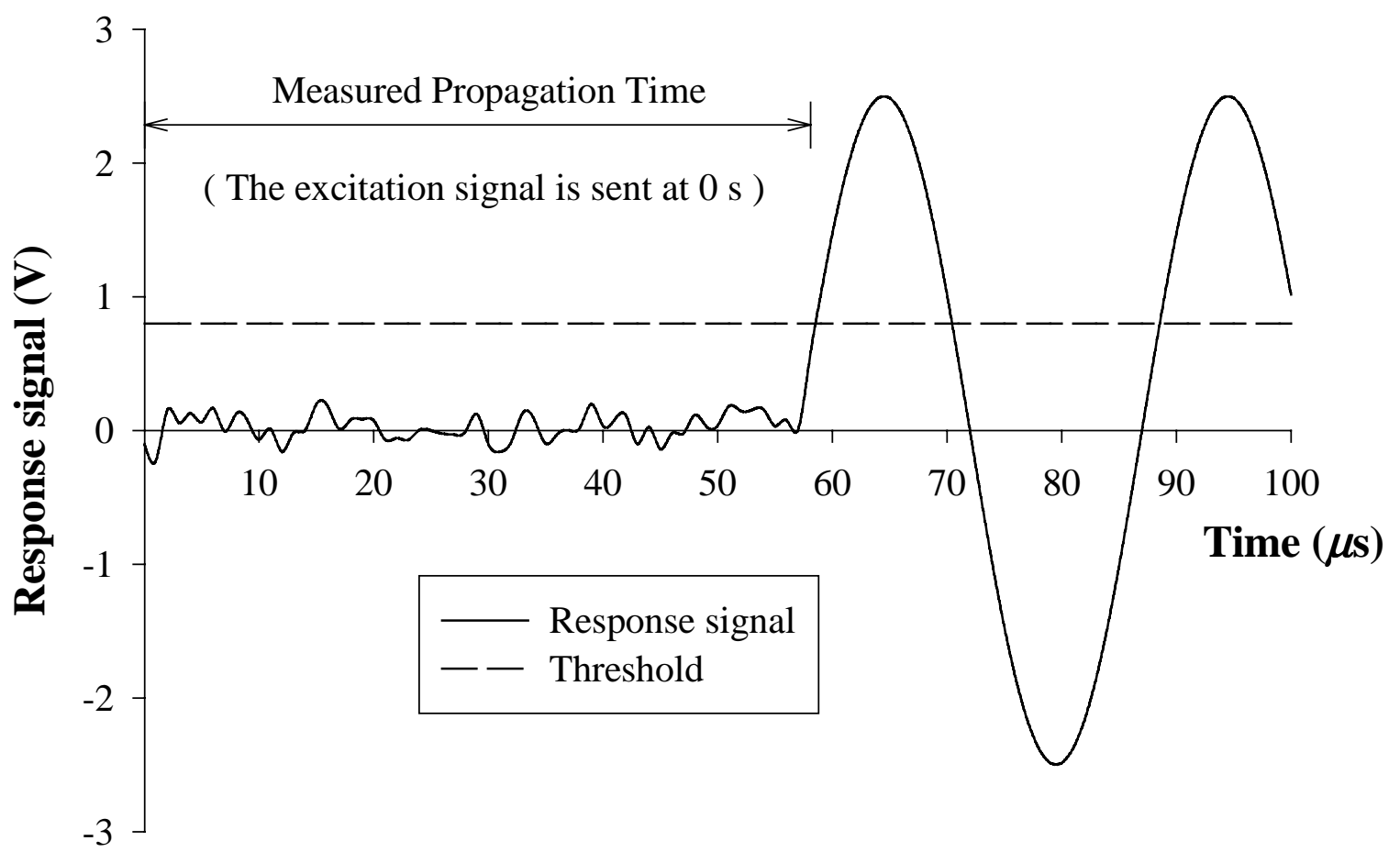

Figure 5.7 - Operation principle of the ultrasonic timer 
Figure 5.7 demonstrates the operation principle of the ultrasonic timer. Timing starts when the excitation impulse rises, and stops when the received signal reaches a threshold value, above the noise level. The advantage of this method is that there is minimal delay between the reception of the signal and the stoppage of the timer. The measured time must be corrected to account for travelling time in the transducer house.

Preliminary measurements were executed on veneer sheets, using various grain orientations. The exact location of the accelerometers was marked, and thin veneer strips were cut from the sheet, to include the marked measurement locations. Propagation times measured on veneer strips were no different from those obtained by measuring large sheets. This led to the conclusion that Equation 4.15 is sufficient for dynamic MOE determination.

Specimen manufacturing and testing included the following steps:

- Cutting structural veneer sheets to target dimensions of approx. 200 x $200 \mathrm{~mm}$;

- $\quad$ Specimen conditioning (see section 5.1.2);

- Taking two width and length and four thickness measurements to $0.01 \mathrm{~mm}$ accuracy;

- Measuring weight to the nearest $0.01 \mathrm{~g}$;

- Marking both sides of the sheets. Drawing lines at $0^{\circ}$ to $90^{\circ}$ angle to the grain, with $15^{\circ}$ increment, to designate the measurement directions;

- Measuring the propagation time of ultrasound in the directions marked.

Data analysis involved calculating the density of the specimens, and the propagation time (average of the corresponding measurements on the two sides) in each direction on the sheets. The propagation velocity was calculated by dividing the 


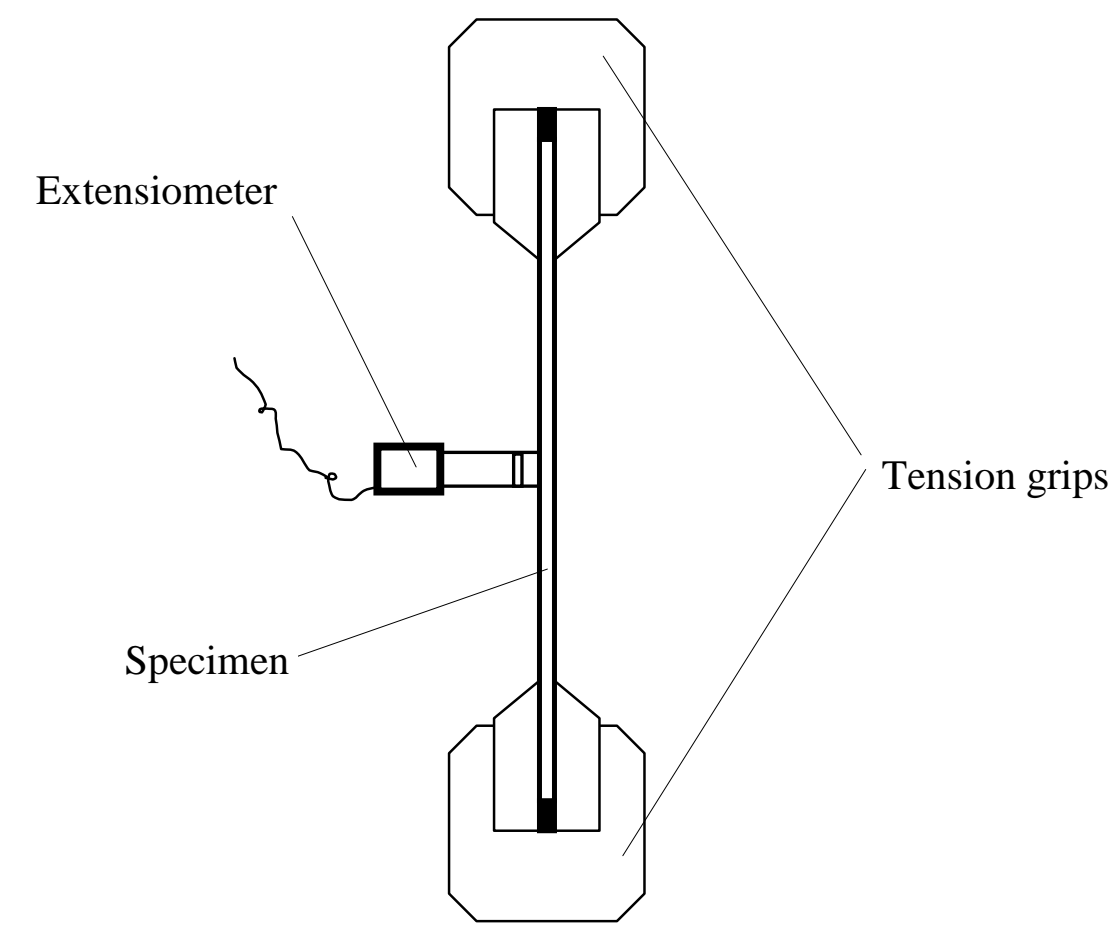

a.

Figure 5.8 - Schematic of the static tension test and the experimental setup 
transducer distance with the propagation time. Finally, Equation 4.15 provided the $E_{D}$ values. Since every specimen was tested in each of the seven measurement directions, this situation corresponds to a randomized complete block (RCB) design.

Relationship between the dynamic and static MOE was established by testing several veneer and solid wood specimens of each species (conditioned according to section 5.1.2), at four grain angle levels $\left(0^{\circ}, 15^{\circ}, 30^{\circ}\right.$ and $\left.45^{\circ}\right)$. The target dimensions were $300 \mathrm{~mm}$ in length and $25 \mathrm{~mm}$ in width. Solid wood specimens were $12 \mathrm{~mm}$ thick. Dynamic MOE was measured on these specimens in a similar manner as described above. Static MOE was measured using the MTS servo-hydraulic testing machine, equipped with mechanical tension grips. Load and strain values were collected using a computerized data acquisition system, with a data collection frequency of 1s. Figure 5.8 shows the measurement setup of the static tension test. Testing speed and other testing parameters were in accordance with the standard ASTM 143 - 94.

\subsection{Densification}

The effect of the density increase on the MOE of veneer was tested using the stress-wave timer, and the same specimens that had been used to assess the orthotropic tensile MOE. Preparation of the specimens included densification of the veneer sheets in a small scale laboratory press for 15 minutes. The temperature of the heated platens was $120^{\circ} \mathrm{C}\left(250^{\circ} \mathrm{F}\right)$. Densification pressures varied so as to cause different levels of compaction, typically between 10 and $80 \%$. After densification, veneer sheets were placed in a controlled environment of $21^{\circ} \mathrm{C}$ and $65 \% \mathrm{RH}$ for several days prior to testing. 
Specimen testing procedure was similar to that described in section 5.1.5. Length and width measurements were omitted; these dimensions were assumed unchanged. Stress-wave propagation directions corresponded to the previously marked lines, used for orthotropic tensile MOE determination. Because the original dynamic MOE values in these directions were already available, this made the calculation of a percent MOE increase possible, for every direction in each veneer sheet, individually.

A small-scale static densification study was also conducted, to verify the capacity of the dynamic test to estimate the effect of densification on the static MOE of veneers. This study involved ten, 300 by $300 \mathrm{~mm}^{2}$ yellow-poplar veneer sheets, that were cut, parallel to the grain, into $25 \mathrm{~mm}$ wide strips, and marked for the identification of the original sheet. Strands were randomly assigned into groups, containing one strand from each sheet. Each group went through densification, using the same pressing parameters as described above, and different densification pressures, to achieve different levels of compaction for each group. One group was retained as an undensified control.

After a conditioning period of several days $\left(21^{\circ} \mathrm{C}, 65 \% \mathrm{RH}\right)$, a static testing procedure, as described in section 5.1.5, established the tensile MOE of each veneer strip. The level of densification was calculated from the original and densified thickness of each veneer strand. The MOE of the undensified control specimen originating from the same veneer sheet provided a basis for computing the MOE increase of each densified specimen. 


\subsection{Mechanical properties of the composites}

\subsubsection{Raw materials}

In order to validate the applicability of orthotropic models and the predictions of the simulation models described in chapter 7, two commercially available composite lumber materials were tested for various mechanical parameters:

1. 15-layer yellow-poplar Laminated Veneer Lumber, manufactured from $3.2 \mathrm{~mm}$ (1/8 in) thick peeled structural veneer sheets. The LVL beams were produced at TrusJoist, a Weyerhaeuser Business, Buckhannon, WV, using a continuous technology and crushed lap joints to connect the consecutive veneers. Veneer sheets are sorted by stress-wave timing to ensure uniform product properties, but the layup is not optimized for maximum flatwise bending MOE.

2. Parallel Strand Lumber manufactured at the same facility, using a mix of $75 \%$ yellow-poplar and 25\% southern yellow pine (Pinus spp.) strands. The lumber is produced as large billets, using a continuous technology, at relatively high levels of densification. The billets are later re-sawn into smaller cross-sections.

Figure 5.9 shows the structure of the composites, and defines three orthogonal axes; $x$ is the longitudinal direction of the composites, $y$ is the orientation of the constituents within the cross-section, and $z$ is perpendicular to both. One can realize that these axes are analogous with the longitudinal, tangential and radial directions in solid wood, respectively, especially in LVL, where the anatomical orientation of the veneer layers concur with the length, width and thickness of the beams. 

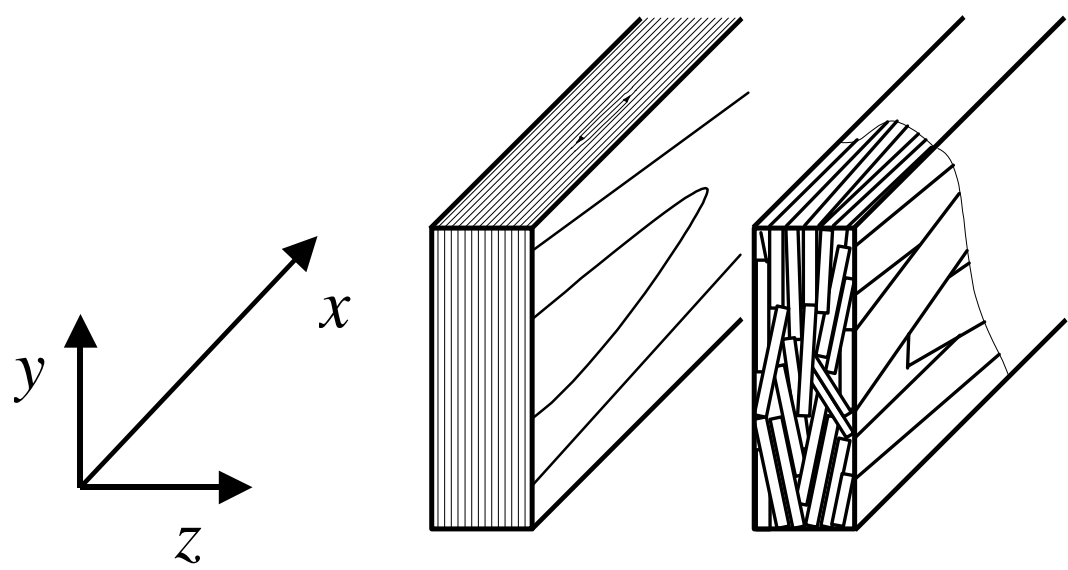

Figure 5.9 - The definition of orthogonal axes for LVL and PSL

The assessment of the orthotropic properties of LVL and PSL requires the definition of two angles. By substituting the $x, y$ and $z$ coordinate axes for $L, T$ and $R$, respectively, on Figures $\mathbf{4 . 1}$ and $\mathbf{4 . 5}$, it is possible to define load orientation $\left(\varphi^{\prime}\right)$ and strand/layer orientation $\left(\theta^{\prime}\right)$. These angles are analogous to grain and ring orientation in solid wood, respectively. Using $\varphi^{\prime}$ and $\theta^{\prime}$, it is possible to examine the compression and shear orthotropy of composite lumber in the same way as that of solid wood.

\subsubsection{Bending MOE measurements}

Twenty structural size beams of both LVL and PSL were tested in 4-point bending. The approximate beam cross-sections of LVL and PSL were 45 by $95 \mathrm{~mm}$ and 75 by $140 \mathrm{~mm}$, respectively, and beam length was $2.5 \mathrm{~m}$. LVL specimens consisted of 4 groups of 5 beams manufactured from the same panel, which allowed the determination of both within-panel and between-panel variation. The exact position of the PSL beams in 

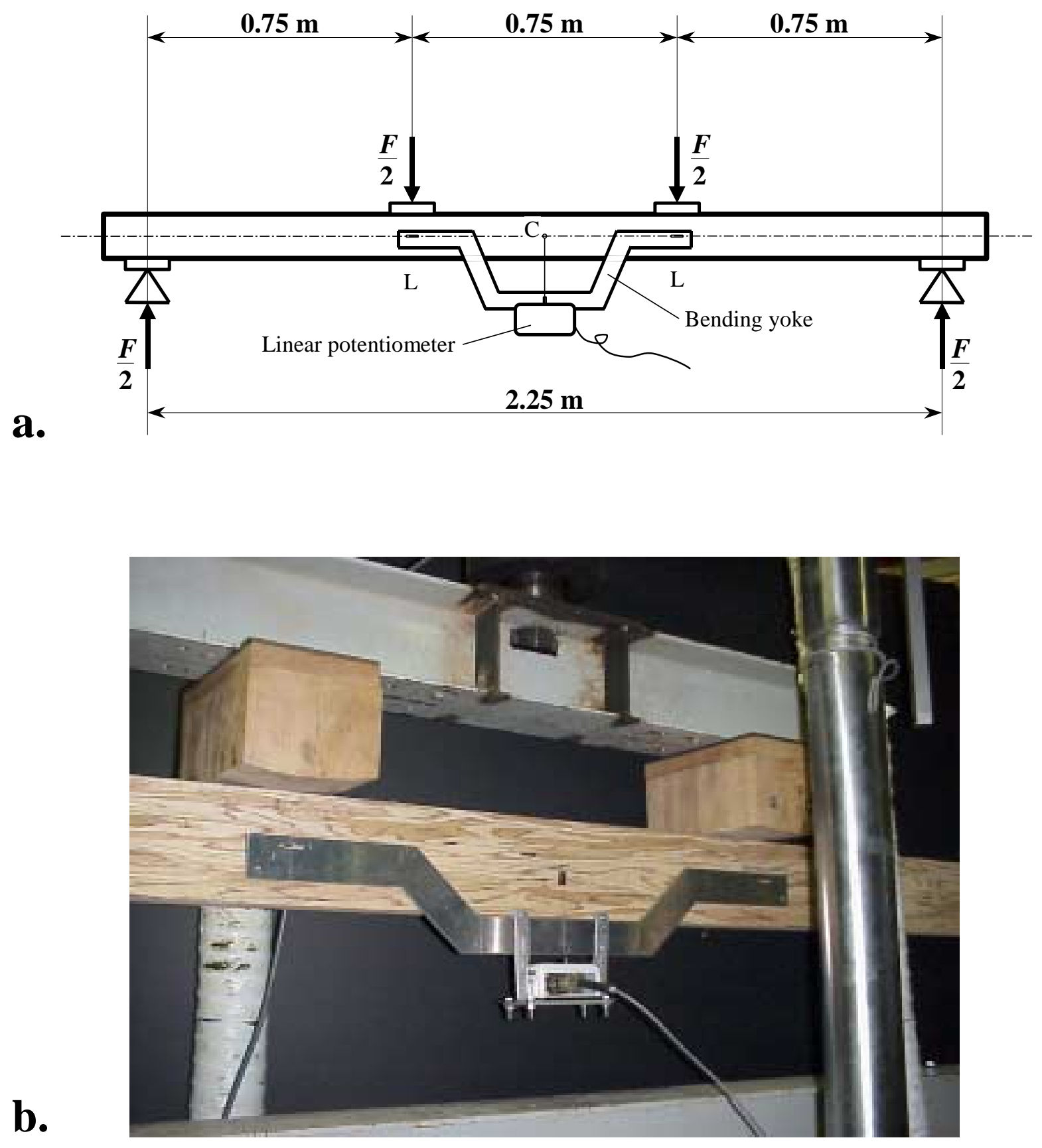

Figure 5.10 - Experimental setup of the composite bending tests 
the production flow was uncertain, and the specimens were treated as a representative random sample of the population. A conditioning period of several weeks in an environment of $21^{\circ} \mathrm{C}$ and $45 \% \mathrm{RH}$ preceded the bending tests. Measurements were carried out using a high-capacity Baldwin universal hydraulic testing machine, equipped with a $220 \mathrm{kN} \pm 5 \mathrm{~N}$ load cell. Testing procedure included both edgewise and flatwise load application for every beam. The applied load never exceeded the linear elastic limit of the beams.

Figure 5.10 shows the testing setup of the bending test. Beam supports consisted of load bearing rollers, one of which provided free lateral adjustment and longitudinal movement for the beams. The load applicators, mounted on a self-aligning crossbar, had a $650 \mathrm{~mm}$ radius of curvature. The vertical displacement of the center point (C) relative to the load application points (L) was measured on the middle plane of the beam, using a linear potentiometer mounted on a bending yoke, which was supported on nails driven into the beam (see Figure 5.10.) The linear potentiometer had a measuring range of 0 $125 \mathrm{~mm}$, and an accuracy of $0.01 \mathrm{~mm}$. A computerized data acquisition system collected load and deflection data with a frequency of $1 / \mathrm{s}$. In the absence of specific testing standards for wood-based composite lumber, the location of the load application points and the testing speed were established in accordance with ASTM D 198 - 94. The evaluation of the collected data followed the specification of this standard, too. 


\subsubsection{Orthotropy of shear strength}

The orthotropic shear strength of LVL and PSL was measured to verify the applicability of the models described in section 4.1.1, for these composites. The orientation of the sheared plane and the direction of load application involved combinations similar to the ones used for solid wood (see section 5.1.3). In this case, however, not all combinations were measured. Shear measurements included all planes for the longitudinal $(x)$ direction $\left(\varphi^{\prime}=0^{\circ} ; \theta^{\prime}=0^{\circ}, 15^{\circ}, \ldots 90^{\circ}\right)$, and load applications in every direction in the $x y$ and $x z$ planes. $\left(\varphi^{\prime}=0^{\circ}, 15^{\circ}, \ldots 90^{\circ}\right.$ for $\theta^{\prime}=0^{\circ}$ and $90^{\circ}$.) Shear strength was also determined at $\varphi^{\prime}=90^{\circ} ; \theta^{\prime}=45^{\circ}$, to furnish Equations 4.1, 4.4 and 4.5 with every required constant.

The sample size was 9 specimens for each combination. Instead of the testing setup shown on Figure 5.3, the standard ASTM testing apparatus was used (ASTM D 143 -94). The target shape and size of the specimens were also those specified in the standard, although beam cross-section sometimes limited the dimensions of the specimens. Other details of the measurements, including specimen conditioning, testing speed and the testing machine, were the same as specified in section 5.1.3.

\subsubsection{Orthotropy of compression elasticity}

The orthotropic compression properties of the composites were assessed to verify the simulation models that estimate the compression MOE of LVL and PSL in different directions. Composite MOE was measured only in the six directions that are required for the prediction of the Orthotropic Tensor Theory $\left(E_{x}, E_{y}, E_{z}, E_{45^{\circ}}^{0^{\circ}}, E_{45^{\circ}}^{90^{\circ}}, E_{90^{\circ}}^{45^{\circ}}-\right.$ see 
Equation 4.10), using a sample size of 10 in each direction. Specimen preparation and testing procedures were the same as described in section 5.1.4, with two notable exceptions:

- Available composite beam thickness did not allow the manufacture of $100 \mathrm{~mm}$ long specimens in directions that deviated from the $x y$ plane. In these instances specimen manufacture included re-gluing the composite lumber with cold-setting Polyvinyl Acetate (PVAc) wood adhesive, to create beams with double thickness.

- For LVL, the maximum thickness created using the above practice was $87 \mathrm{~mm}$, which restricted the maximum length of LVL compression in the $y z$ and $z$ directions. For these specimens, the gauge length of the strain measurement decreased to $35.5 \mathrm{~mm}$.

\subsection{Composite geometry}

Accurate simulation of the elastic properties of LVL and PSL required the determination of certain geometric parameters of these composites, as well as those of the raw material before composite manufacture. Some of these variables can be described by a constant value (i.e. deterministic parameters), while others can assume random values, and can only be characterized by a statistical distribution (stochastic variables.)

\subsubsection{Geometric properties of the raw material}

The original thickness $\left(t_{o}\right)$ of the veneer sheets in the layup of LVL before hot pressing is a stochastic variable. This variable was measured for all three species, with an accuracy of $0.01 \mathrm{~mm}$, during the measurement of dynamic MOE. This yielded 80-90 thickness measurements for each species. Since these veneer sheets had been peeled in a 
commercial composite lumber manufacturing facility, measured thickness data is representative of the typical original veneer thickness, and can be used to establish the statistical distribution of $t_{o}$.

The constituents of PSL are veneer strands that were cut out of the same veneer sheets described above, thus the distribution of their $t_{o}$ values will follow the same probability density functions. The width of these strands is a stochastic parameter, with a mean of $25 \mathrm{~mm}$ (1 in.) As a simplification, this value was treated as a deterministic parameter.

\subsubsection{Geometric properties of LVL}

The geometry of LVL includes three stochastic and one deterministic parameter (Figure 5.11 a.) The stochastic parameters are the length of the veneer crushed lap joints or overlaps $(\lambda)$, distance between two subsequent overlaps $(\delta)$, and layer thickness. The deterministic variable is the number of layers, which - for the particular composite used in this study - was 15 in areas that did not contain overlap, and 16 where an overlap occured.

Overlap lengths and distance determination included $145 \lambda$ and $145 \delta$ values, measured on the longitudinal sections of LVL beams, using calipers. Layer thickness was assessed by analyzing high-resolution computer images, of longitudinal sections. The analysis involved scanning 11 overlap-free sections of full-thickness LVL pieces, pasting a digital 'ruler' over the scanned images to facilitate high-accuracy layer thickness measurements, and recording the thickness of each layer (Figure 5.12). 


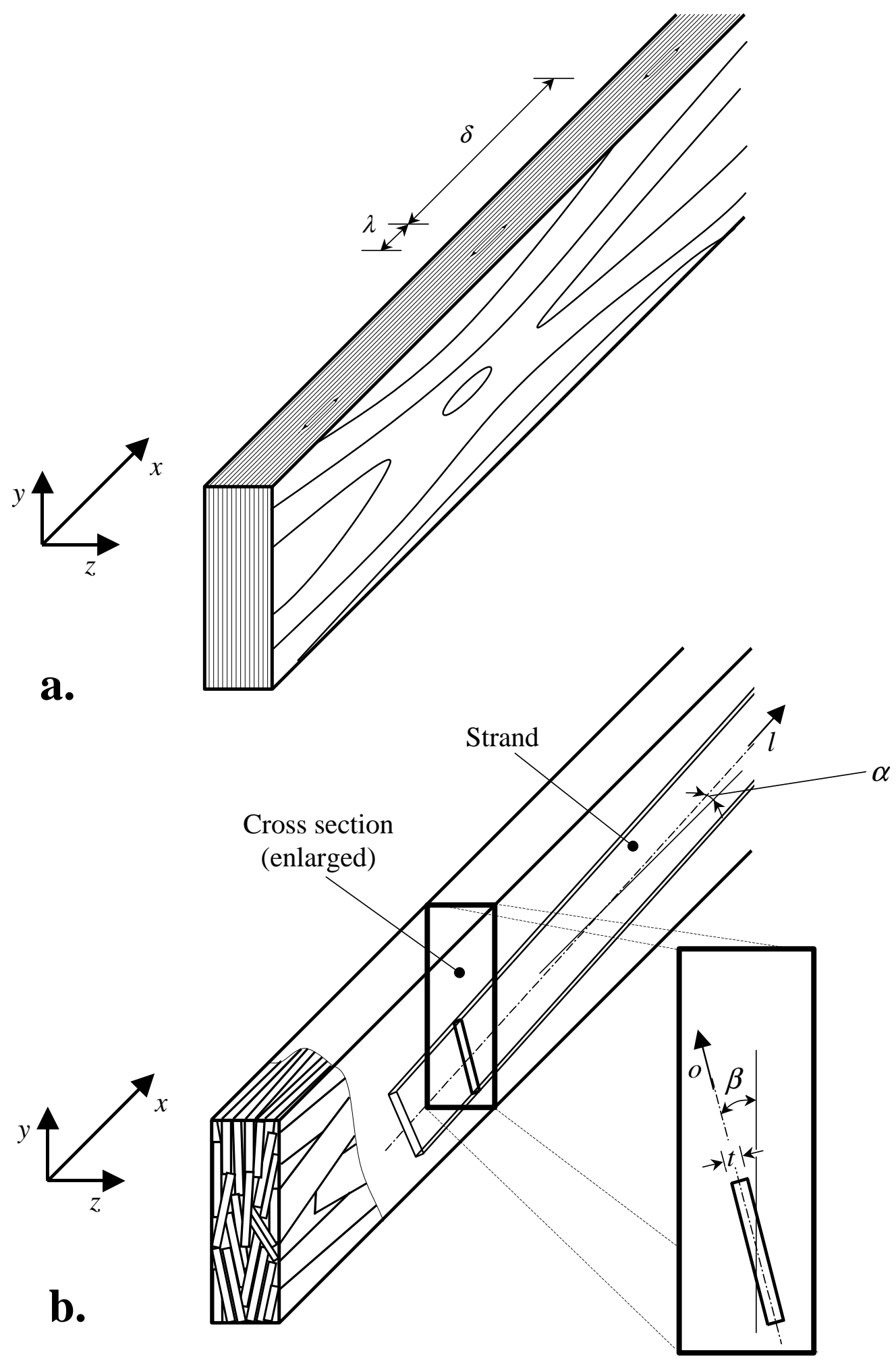

Figure 5.11 - Geometric structure and stochastic parameters of LVL (a) and PSL (b) 


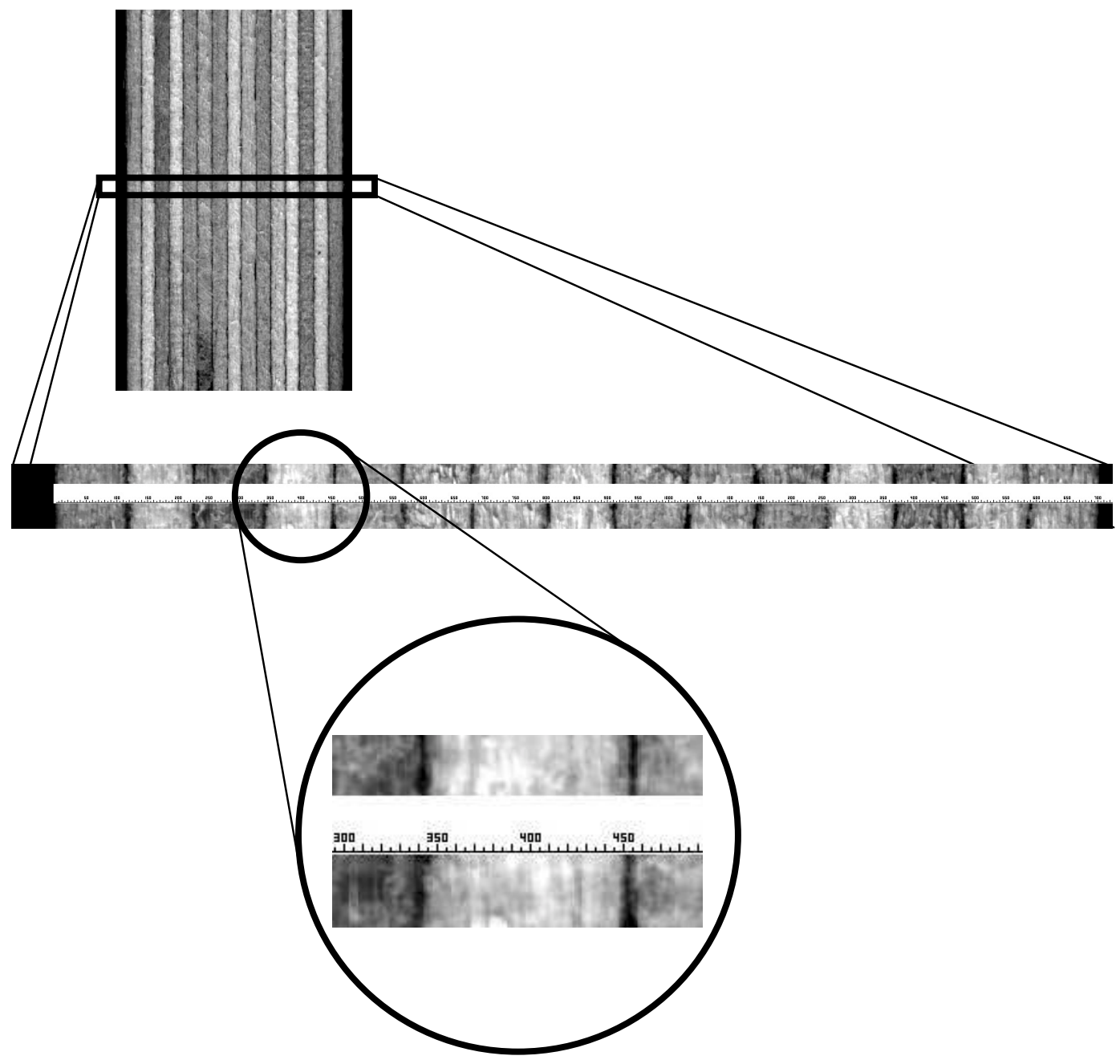

Figure 5.12 - Digital images used for LVL layer thickness measurements

\subsubsection{Geometric properties of PSL}

The geometric structure of PSL includes four stochastic parameters (Figure 5.9 b):

- Strand angle $(\alpha)$ is the angle between the longitudinal axis of the beam $(x)$ and that of the strand $(l)$. The deviation of the strand axes from the $x y$ plane, which is typically very small in reality, was disregarded. A protractor provided strand angle measurements on the $x y$ section of PSL beams, to the nearest degree. 
- Strand deviation $(\beta)$ is the angle between the general cross-sectional orientation of the strands $(y)$, and that of the particular strand $(o)$. Since strands are usually bent and/or distorted in the cross-section, the approximate deviation was measured on images of beam cross-sections, to the nearest angle, using a protractor.

- Projected strand thickness $(t)$ was also measured on cross-sectional images, using digital calipers. Since the thickness of the same strand may vary in the cross-section, the mean of five measurements provided the average projected strand thickness.

- In PSL, the number of strands in a unit cross-section $(u)$ is also a random variable. The determination of $u$ involved counting the number of strands in ten cross-sections, and dividing the count by the cross-sectional area in every case. The count included partial strands only if their centroid appeared to be within the cross-sectional boundaries.

Cross-sections used for determining $\beta, t$ and $u$ originated from different PSL boards to ensure the independence of the measured data.

\subsubsection{Data analysis}

A sufficiently high number of measurements were collected of each of the stochastic parameters, to allow statistical distribution determination. The ExpertFit ${ }^{\circledR}$ statistical analysis program (Law and Vincent 1999) was used to find the best probability density function to describe the geometric parameters. In choosing the best statistical distribution function, the following considerations were used (in order of importance):

- Good data representation was the most important criteria. The function ranked highest by ExpertFit ${ }^{\circledR}$ usually received preference;

- Ease of application in a simulation model was an important factor when choosing for equally representative models; 
- Other factors were less critical. For example, if an unbounded distribution fit the experimental data best, it was preferred, even though using it in a simulation might yield unrealistic figures (e.g., negative thickness values.) Although the probability of getting negative values is usually extremely small, simulation routines using these functions should include provisions to exclude such results. 


\section{RESULTS AND DISCUSSION}

\subsection{Orthotropy of the raw material}

\subsubsection{Physical properties of the raw materials}

Table 6.1 shows the measured physical properties of the solid wood specimens, as well as the veneer sheets manufactured from the Appalachian species. In addition to moisture content (MC) and specific gravity (SG), weight density is also reported for the veneer specimens. Veneer density statistics are necessary for the validation of the developed simulation models.

Table 6.1 - Summary statistics of the measured physical properties of Appalachian hardwood species

\begin{tabular}{|c|c|c|c|c|c|c|c|}
\hline \multirow[b]{2}{*}{ Species } & \multirow[b]{2}{*}{$\mathbf{N}^{\mathrm{a}}$} & \multicolumn{2}{|c|}{$\begin{array}{c}\text { Moisture Content } \\
(\%)\end{array}$} & \multicolumn{2}{|c|}{ Specific gravity } & \multicolumn{2}{|c|}{$\begin{array}{l}\text { Density } \\
\left(\mathrm{kg} / \mathbf{m}^{3}\right)\end{array}$} \\
\hline & & $\overline{\mathbf{x}}^{\mathrm{b}}$ & $\mathbf{S}^{\mathrm{c}}$ & $\overline{\mathbf{x}}$ & $\mathbf{S}$ & $\overline{\mathbf{x}}$ & $\mathbf{S}$ \\
\hline \multicolumn{8}{|l|}{ Solid wood } \\
\hline Aspen & 10 & 11.4 & 0.92 & 0.39 & 0.01 & -- & -- \\
\hline Red oak & 10 & 11.1 & 0.30 & 0.63 & 0.03 & -- & -- \\
\hline Yellow-poplar & 10 & 11.3 & 0.51 & 0.39 & 0.01 & -- & -- \\
\hline \multicolumn{8}{|l|}{ Veneer } \\
\hline Aspen & 20 & 11.8 & 0.32 & 0.37 & 0.01 & 417 & 16 \\
\hline Red oak & 20 & 10.5 & 0.38 & 0.50 & 0.01 & 552 & 14 \\
\hline Yellow-poplar & 20 & 11.5 & 0.61 & 0.42 & 0.02 & 469 & 24 \\
\hline
\end{tabular}




\subsubsection{Orthotropy of shear strength}

Table 6.2 summarizes the basic statistics of all the experimentally obtained shear strength data by species. The mean values were used to create anisotropy diagrams in three dimensional Cartesian coordinate systems as shown on Figures 6.1 a through 6.3 a. The intermediate grid data points were generated by inverse distance interpolation using a commercial software SigmaPlot $^{\circledR}$ (SPSS Inc. 1997) for better viewing.

In general, shear strength decreased significantly ${ }^{\dagger}$ with the increase of grain angle for all species involved in the study. At zero degree grain angle (traditional shear, parallel to the grain) the shear strength decreased slightly as ring angle increased from 0 to 90 degrees. However, this tendency could not be observed at fixed $90^{\circ}$ grain angle (i.e., rolling shear). Either a slight increase or local maximum was experienced.

Maximum shear strength values (MSS) were not consistently measured at $0^{\circ}$ grain orientation. At $0^{\circ}$ ring angle for all the species, the MSS was measured at $15^{\circ}$ grain orientation. Other researchers reported the same phenomenon (Ashkenazi 1959; Szalai 1994). This characteristic may be explained by the study of stress distribution function along the length of the sheared plane. Yilinen (1963) demonstrated that this function depends on the length to width ratio of the sheared plane and other factors including force application method, etc. It might be suspected that the stress distribution at $15^{\circ}$ grain orientation becomes more uniform along the length of the sheared plane, while possible stress peaks near the entrance notch at $0^{\circ}$ grain angle accelerate the failure.

\footnotetext{
$\dagger$ Statistical significance was assessed at a 95\% confidence level $(\mathrm{p}<0.05)$
} 
Table 6.2 - Summary and basic statistics of the experimentally determined shear strength values

\begin{tabular}{|c|c|c|c|c|c|c|c|c|c|c|c|c|c|c|c|c|c|c|c|c|c|}
\hline \multicolumn{22}{|c|}{ ASPEN } \\
\hline \multirow{4}{*}{$\begin{array}{c}\text { Grain } \\
\text { angle } \\
\left(\varphi^{\circ}\right)\end{array}$} & \multicolumn{21}{|c|}{ Ring Orientation $\left(\theta^{\circ}\right)$} \\
\hline & \multicolumn{3}{|c|}{$\mathbf{0}$} & \multicolumn{3}{|c|}{15} & \multirow{2}{*}{\multicolumn{2}{|c|}{30}} & \multirow{2}{*}{\multicolumn{3}{|c|}{$\begin{array}{c}45 \\
\text { Shear Stren }\end{array}$}} & & \multicolumn{3}{|c|}{60} & \multicolumn{3}{|c|}{75} & \multicolumn{3}{|c|}{90} \\
\hline & \multicolumn{16}{|c|}{ Shear Strength } & & & & & \\
\hline & $\mathbf{N}^{\mathbf{a}}$ & $\bar{\tau}^{b}$ & $\mathbf{s}^{\mathrm{c}}$ & $\mathbf{N}$ & $\bar{\tau}$ & $\mathbf{S}$ & $\mathbf{N}$ & $\bar{\tau}$ & $\mathbf{S}$ & $\mathbf{N}$ & $\bar{\tau}$ & $\mathbf{S}$ & $\mathbf{N}$ & $\bar{\tau}$ & $\mathbf{S}$ & $\mathbf{N}$ & $\bar{\tau}$ & $\mathbf{S}$ & $\mathbf{N}$ & $\bar{\tau}$ & $\mathbf{S}$ \\
\hline 0 & 15 & 7.87 & 0.23 & 15 & 7.30 & 0.50 & 15 & 6.77 & 0.46 & 15 & 5.42 & 0.79 & 15 & 6.16 & 0.64 & 15 & 6.17 & 0.27 & 15 & 6.30 & 0.80 \\
\hline 15 & 9 & 8.39 & 0.38 & 8 & 8.16 & 1.05 & 9 & 7.98 & 0.93 & 8 & 7.30 & 1.03 & 7 & 6.67 & 0.84 & 8 & 5.78 & 0.40 & 9 & 5.99 & 0.60 \\
\hline 30 & 10 & 7.16 & 0.32 & 8 & 6.37 & 0.90 & 7 & 7.21 & 0.80 & 8 & 5.74 & 0.47 & 7 & 5.62 & 1.03 & 8 & 4.99 & 1.45 & 7 & 5.62 & 0.80 \\
\hline 45 & 6 & 4.98 & 0.38 & 6 & 4.29 & 0.34 & 6 & 4.75 & 0.30 & 6 & 4.27 & 0.24 & 6 & 4.44 & 0.24 & 6 & 5.02 & 0.57 & 6 & 4.42 & 0.22 \\
\hline 60 & 6 & 3.18 & 0.31 & 6 & 3.45 & 0.45 & 6 & 3.43 & 0.22 & 5 & 3.25 & 0.38 & 5 & 2.54 & 0.50 & 4 & 2.61 & 0.27 & 5 & 3.13 & 0.44 \\
\hline 75 & 6 & 1.92 & 0.12 & 6 & 2.52 & 0.36 & 6 & 2.81 & 0.36 & 6 & 1.93 & 0.14 & 6 & 1.90 & 0.09 & 6 & 2.73 & 0.12 & 6 & 2.45 & 0.24 \\
\hline 90 & 10 & 2.24 & 0.07 & 10 & 1.43 & 0.13 & 11 & 2.24 & 0.09 & 11 & 2.28 & 0.09 & 11 & 3.11 & 0.09 & 10 & 2.98 & 0.09 & 10 & 2.54 & 0.12 \\
\hline
\end{tabular}

OAK

\begin{tabular}{|c|c|c|c|c|c|c|c|c|c|c|c|c|c|c|c|c|c|c|c|c|c|}
\hline \multirow{4}{*}{$\begin{array}{c}\text { Grain } \\
\text { angle } \\
\left(\varphi^{\circ}\right)\end{array}$} & \multicolumn{21}{|c|}{ Ring Orientation $\left(\theta^{\circ}\right)$} \\
\hline & \multicolumn{3}{|c|}{$\mathbf{0}$} & \multicolumn{3}{|c|}{15} & \multicolumn{2}{|r|}{30} & \multicolumn{3}{|c|}{45} & \multicolumn{4}{|c|}{60} & \multicolumn{3}{|c|}{75} & \multicolumn{3}{|c|}{90} \\
\hline & \multicolumn{21}{|c|}{ Shear Strength } \\
\hline & $\mathbf{N}$ & $\bar{\tau}$ & S & $\mathbf{N}$ & $\bar{\tau}$ & $\mathbf{S}$ & $\mathbf{N}$ & $\bar{\tau}$ & $\mathbf{S}$ & $\mathbf{N}$ & $\bar{\tau}$ & S & ' & $\bar{\tau}$ & $\mathbf{S}$ & $\mathbf{N}$ & $\bar{\tau}$ & S & $\mathbf{N}$ & $\bar{\tau}$ & S \\
\hline 0 & 15 & 11.97 & 1.04 & 15 & 12.01 & 0.95 & 15 & 11.6 & 0.48 & 15 & 11.95 & 1.00 & 15 & 12.77 & 0.64 & 15 & 11.36 & 0.65 & 15 & 10.62 & 0.45 \\
\hline 15 & 8 & 11.98 & 0.90 & 9 & 11.28 & 0.67 & 9 & 11.55 & 0.99 & 9 & 12.84 & 0.33 & 9 & 13.04 & 0.89 & 9 & 12.16 & 0.50 & 9 & 12.36 & 0.36 \\
\hline 30 & 8 & 11.85 & 0.74 & 9 & 11.60 & 1.76 & 8 & 11.48 & 1.16 & 8 & 11.36 & 0.91 & 8 & 11.32 & 1.12 & 8 & 11.17 & 1.08 & 8 & 11.33 & 0.50 \\
\hline 45 & 5 & 9.99 & 0.34 & 6 & 9.34 & 0.75 & 5 & 6.64 & 0.75 & 6 & 9.94 & 0.86 & 5 & 8.59 & 0.37 & 5 & 8.38 & 0.50 & 5 & 8.82 & 0.23 \\
\hline 60 & 7 & 7.01 & 0.15 & 7 & 8.07 & 0.39 & 7 & 8.14 & 0.28 & 6 & 7.69 & 0.46 & 7 & 7.20 & 0.21 & 5 & 7.80 & 0.21 & 7 & 6.81 & 0.33 \\
\hline 75 & 7 & 6.06 & 0.30 & 6 & 5.64 & 0.26 & 6 & 5.65 & 0.55 & 7 & 6.41 & 0.43 & 7 & 6.12 & 0.13 & 7 & 6.84 & 0.45 & 6 & 6.57 & 0.22 \\
\hline 90 & 11 & 4.44 & 1.30 & 11 & 5.55 & 0.86 & 11 & 5.72 & 0.73 & 10 & 6.88 & 0.25 & 11 & 7.06 & 0.51 & 10 & 4.62 & 0.87 & 10 & 5.37 & 0.25 \\
\hline
\end{tabular}

YELLOW-POPLAR

\begin{tabular}{|c|c|c|c|c|c|c|c|c|c|c|c|c|c|c|c|c|c|c|c|c|c|}
\hline \multirow{3}{*}{$\begin{array}{c}\text { Grain } \\
\text { angle } \\
\left(\varphi^{\circ}\right) \\
\end{array}$} & \multicolumn{3}{|c|}{$\mathbf{0}$} & \multicolumn{3}{|c|}{15} & \multicolumn{3}{|c|}{30} & \multicolumn{3}{|c|}{45} & \multicolumn{3}{|c|}{60} & \multicolumn{3}{|c|}{75} & \multicolumn{3}{|c|}{90} \\
\hline & \multicolumn{21}{|c|}{ Shear Strength } \\
\hline & $\mathbf{N}$ & $\bar{\tau}$ & $\mathbf{S}$ & $\mathbf{N}$ & $\bar{\tau}$ & $\mathbf{s}$ & $\mathbf{N}$ & $\bar{\tau}$ & $\mathbf{S}$ & $\mathbf{N}$ & $\bar{\tau}$ & S & $\mathbf{N}$ & $\bar{\tau}$ & $\mathbf{S}$ & $\mathbf{N}$ & $\bar{\tau}$ & $\mathbf{s}$ & $\mathbf{N}$ & $\bar{\tau}$ & $\mathbf{S}$ \\
\hline 0 & 15 & 7.91 & 0.49 & 15 & 7.13 & 0.60 & 15 & 7.13 & 0.43 & 15 & 7.32 & 0.49 & 15 & 7.23 & 0.75 & 15 & 9.06 & 0.88 & 15 & 6.18 & 0.30 \\
\hline 15 & 9 & 10.59 & 0.54 & 9 & 8.37 & 1.26 & 9 & 7.31 & 0.80 & 9 & 6.65 & 0.56 & 9 & 6.74 & 0.35 & 10 & 6.77 & 0.35 & 10 & 6.42 & 0.30 \\
\hline 30 & 6 & 6.94 & 0.37 & 8 & 6.70 & 0.74 & 9 & 6.57 & 1.37 & 7 & 6.10 & 1.64 & 9 & 5.91 & 0.85 & 8 & 5.61 & 0.33 & 8 & 5.95 & 1.49 \\
\hline 45 & 5 & 4.85 & 0.19 & 4 & 4.98 & 0.33 & 6 & 4.85 & 0.39 & 6 & 5.74 & 0.82 & 5 & 5.05 & 0.55 & 6 & 4.35 & 0.27 & 5 & 4.73 & 0.92 \\
\hline 60 & 6 & 2.81 & 0.39 & 5 & 3.85 & 0.42 & 6 & 2.81 & 0.10 & 6 & 3.53 & 0.99 & 6 & 3.60 & 0.63 & 6 & 3.13 & 0.31 & 6 & 3.79 & 0.24 \\
\hline 75 & 6 & 2.75 & 0.43 & 6 & 2.96 & 0.42 & 6 & 2.28 & 0.16 & 6 & 2.28 & 0.15 & 6 & 1.94 & 0.06 & 6 & 1.94 & 0.08 & 6 & 2.87 & 0.26 \\
\hline 90 & 3 & 2.43 & 0.68 & 4 & 2.60 & 0.52 & 9 & 2.86 & 0.78 & 11 & 3.52 & 0.54 & 11 & 3.42 & 0.20 & 11 & 3.35 & 0.57 & 11 & 3.17 & 0.43 \\
\hline
\end{tabular}

a - sample size

$\mathrm{b}-$ mean shear strength value $(\mathrm{MPa})$

$\mathrm{c}-$ standard deviation $(\mathrm{MPa})$ 


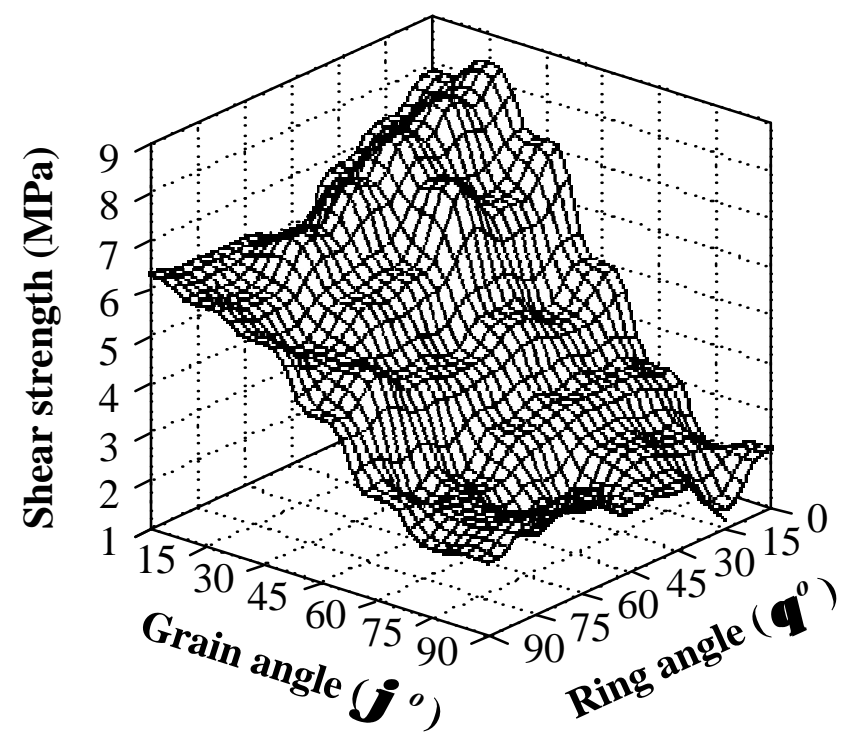

a. - Aspen - Experimental values

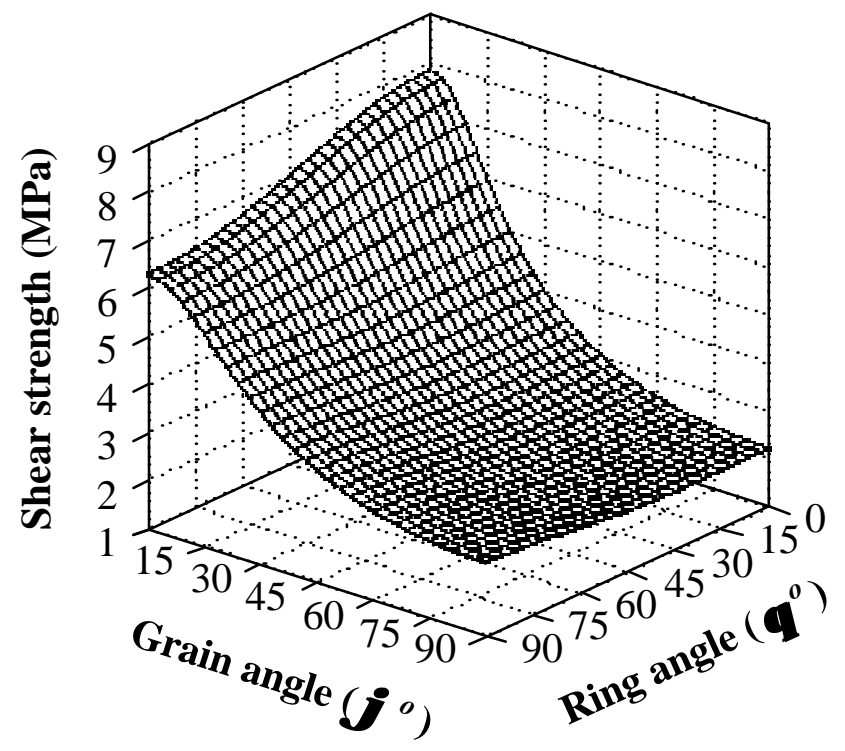

C. - Predicted, Quadratic formula

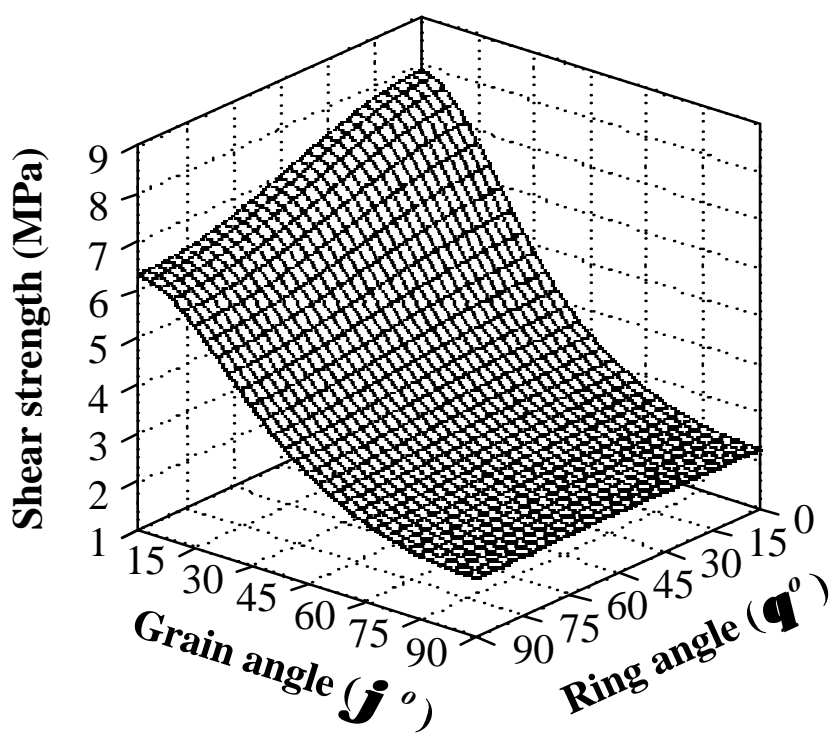

b. - Predicted, Orthotropic Tensor Theory

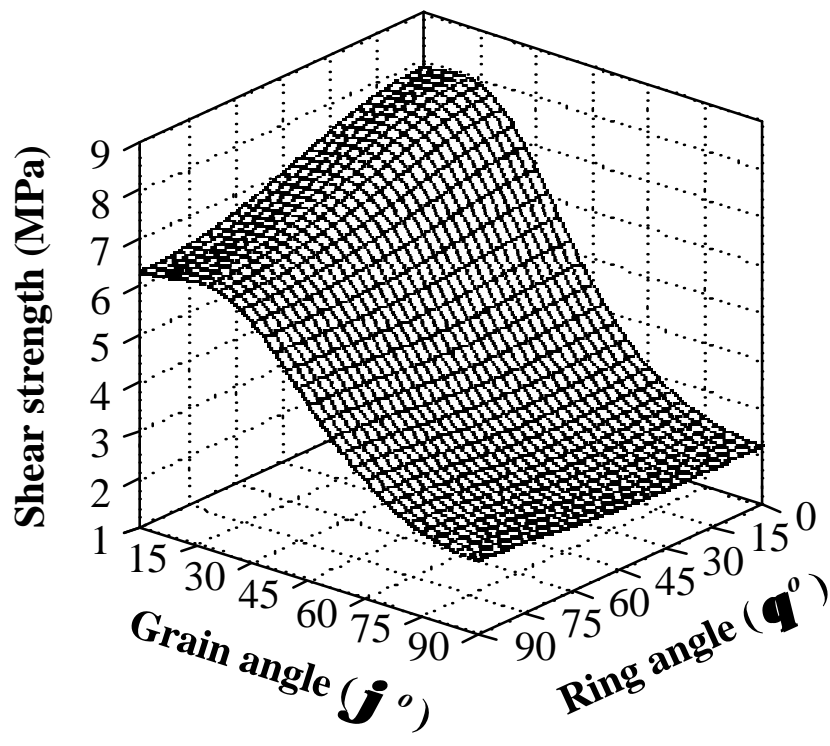

d. - Predicted, Modified Hankinson's formula

Figure 6.1 - Comparison of experimental and model predicted shear strength data of quaking aspen by orthotropic diagrams. 


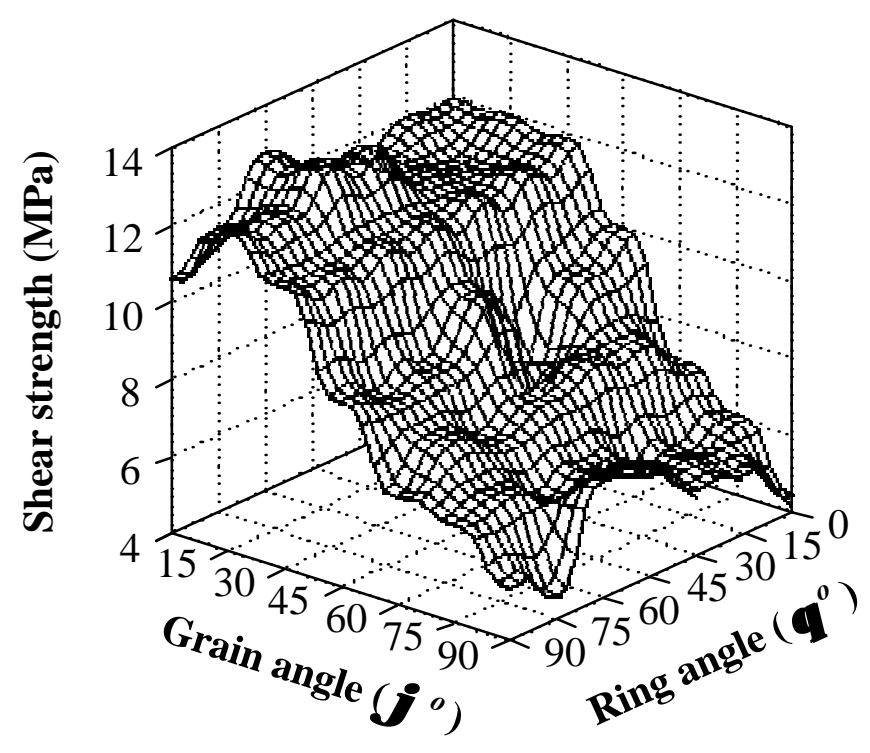

a. - Red oak - Experimental values

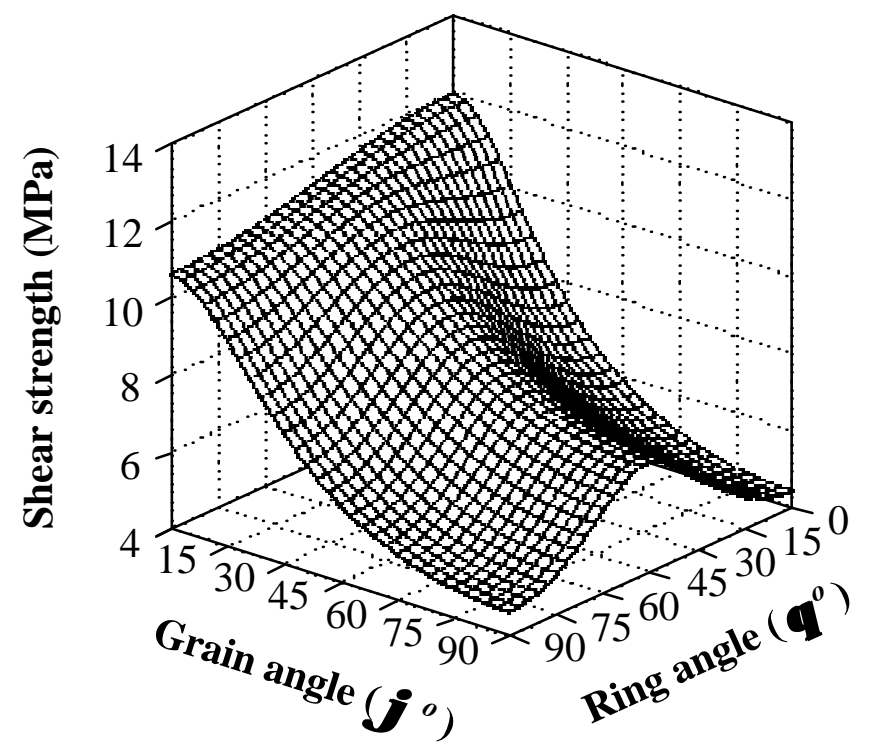

C. - Predicted, Quadratic formula

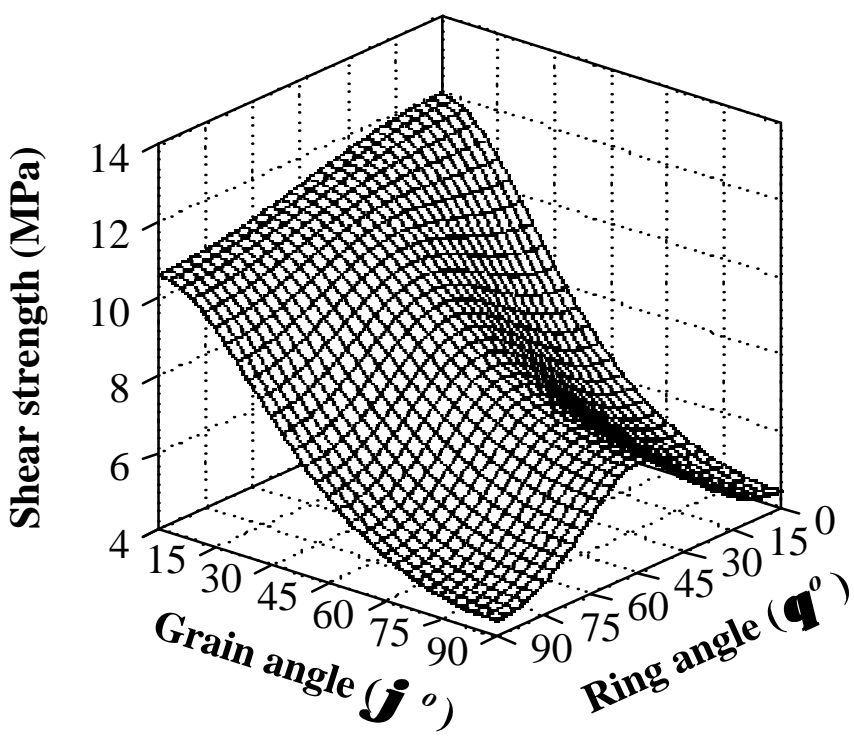

b. - Predicted, Orthotropic Tensor Theory

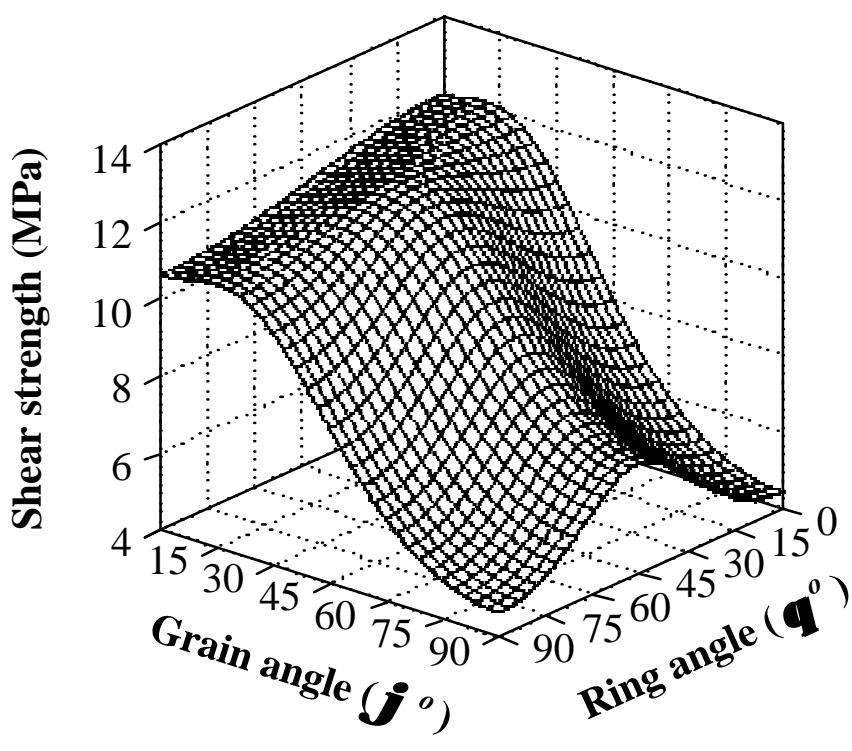

d. - Predicted, Modified Hankinson's formula

Figure 6.2 - Comparison of experimental and model predicted shear strength data of red oak by orthotropic diagrams. 


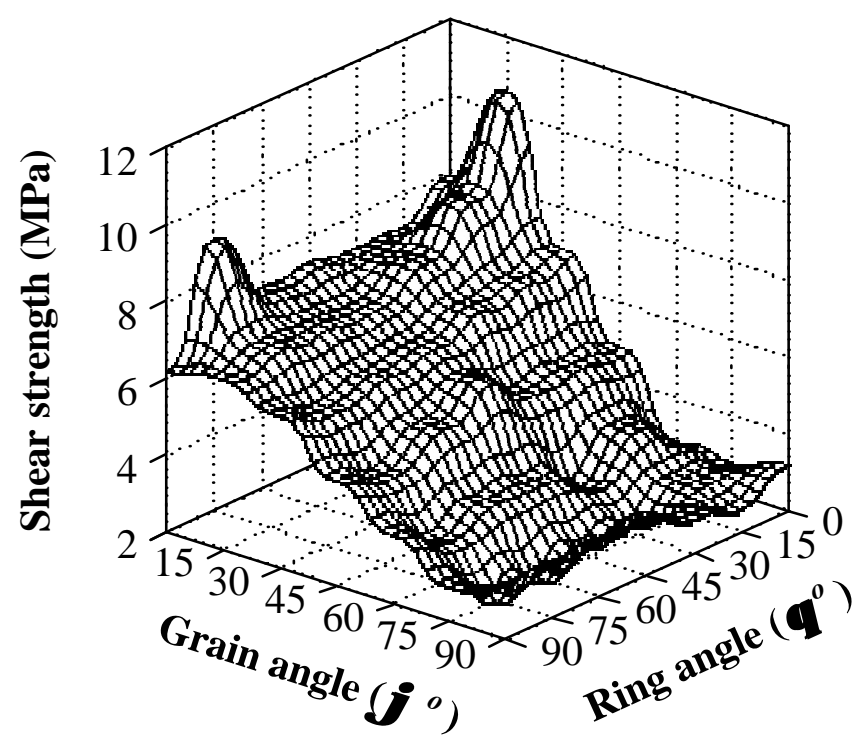

a. - Yellow-poplar - Experimental values

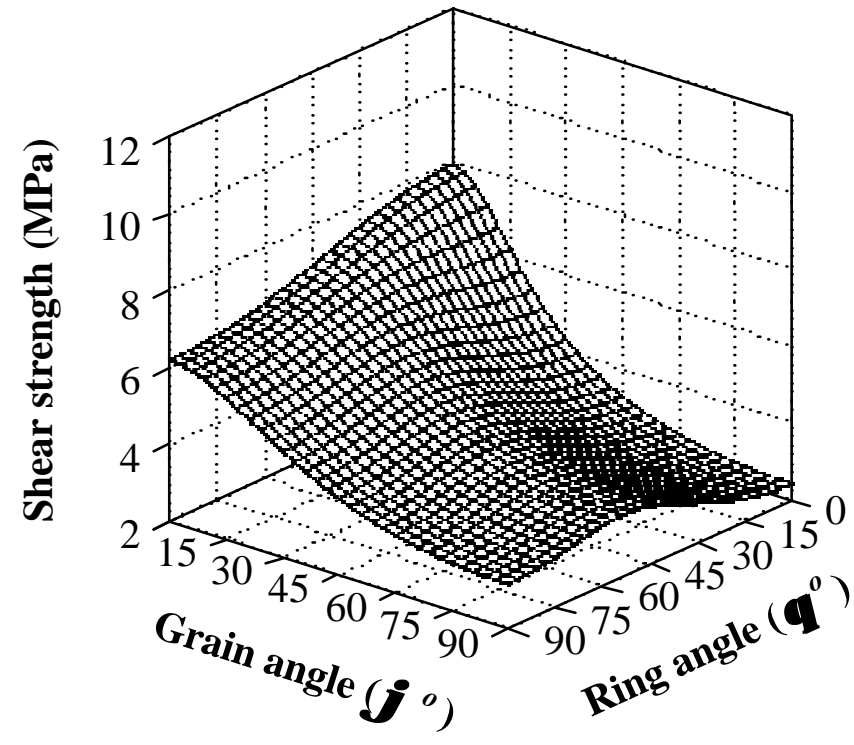

C. - Predicted, Quadratic formula

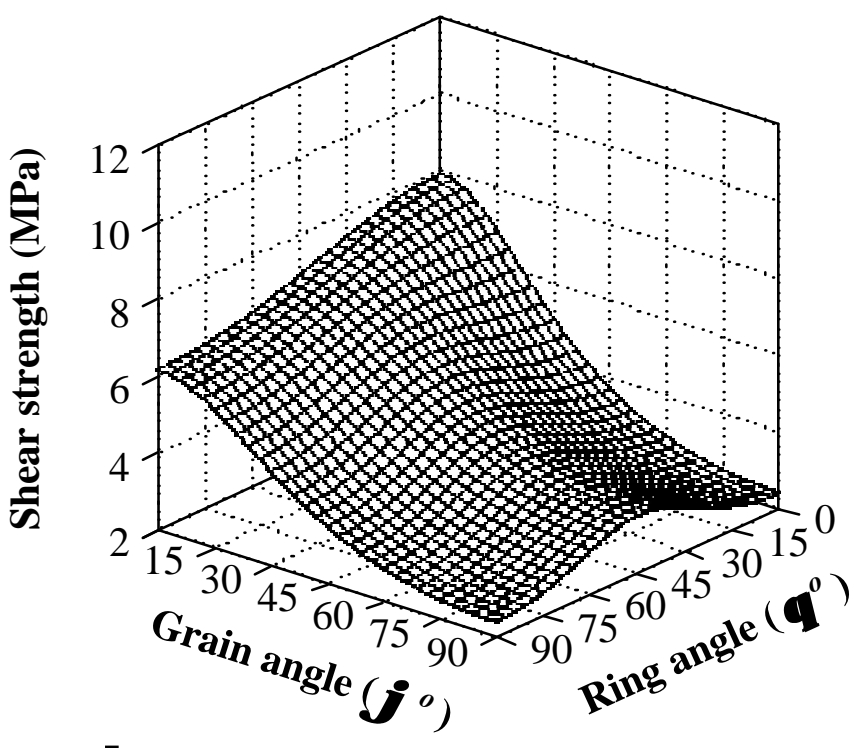

b. - Predicted, Orthotropic Tensor Theory

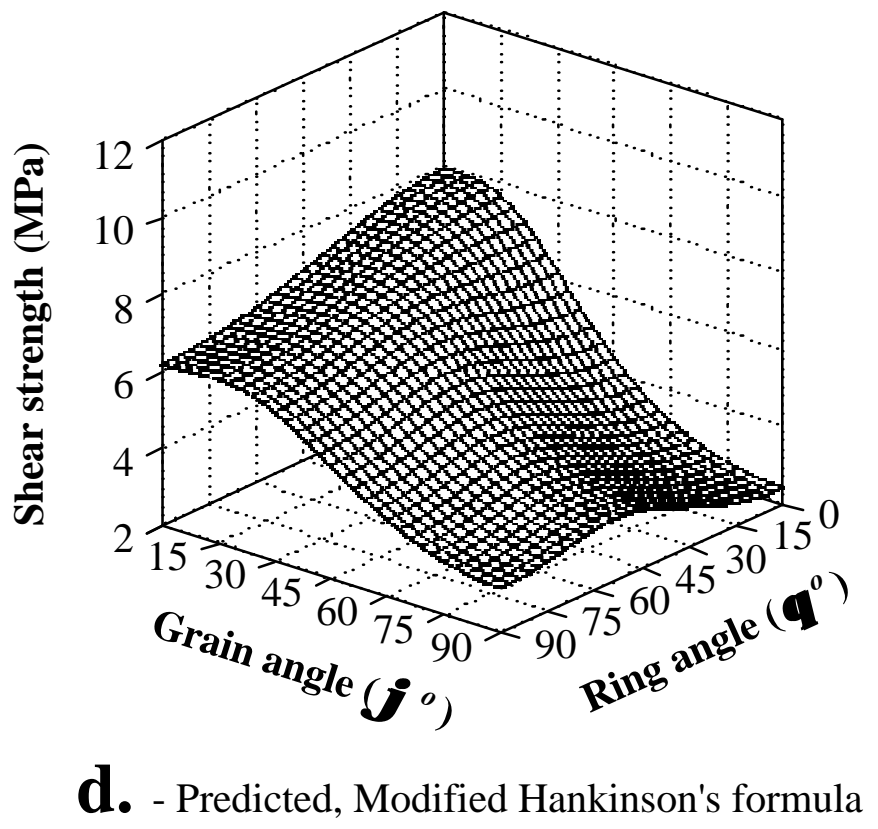

Figure 6.3 - Comparison of experimental and model predicted shear strength data of yellow-poplar by orthotropic diagrams. 
The failure mode was not always pure shear. Over $45^{\circ}$ grain angle, ring-porous wood (oak) inclined to fail along the earlywood/latewood interface or along the ray. The same tendency was encountered regarding the other species as the grain angle approached 90 . Liu and Floeter (1984) observed similar failure modes when testing Sitka spruce specimens in pure shear in the $L T$ plane. They concluded that shear strength depends only on the initiation of failure and not on the direction of fracture propagation. All of the measured shear strength values were kept, even if the specimen failed in a plane that was out of the theoretically sheared plane.

Standard statistical evaluation of the data included two-way ANOVA procedures at $95 \%$ confidence level. The two factors were the grain and ring orientations, both with seven levels according to the $15^{\circ}$ angle increments. The procedure revealed statistically significant differences among the levels of both factors, for all species. Furthermore, significant interaction was detected between the two factors. These results justify the applicability of prediction models that account for the effect of both ring and grain angle on the shear strength. (Detailed ANOVA results are provided in APPENDIX B.)

It should be noted, that all of the data sets exhibited lack of normality and unequal variances. The violation of these statistical assumptions originated from the limited sample size and specimen manufacturing practice. Most of the specimens were cut from the same stem or board and the specimens in a group were machined from blanks consecutively. Thus, complete randomization could not be achieved. More extensive testing and the fulfillment of completely randomized design were beyond the limitations of this research. 
Table 6.3 - Coefficients of determination provided by the various prediction models

\begin{tabular}{lcccc}
\hline \hline \multicolumn{1}{c}{ Species } & $\begin{array}{c}\text { Orthotropic } \\
\text { tensor theory } \\
\mathbf{r}^{\mathbf{2}}\end{array}$ & $\begin{array}{c}\text { Quadratic } \\
\text { formula } \\
\mathbf{r}^{\mathbf{2}}\end{array}$ & \multicolumn{2}{c}{ Modified Hankinson formula } \\
\hline Aspen & 0.73 & 0.59 & 2.72 & $\mathbf{r}^{\mathbf{2}}$ \\
Red oak & 0.61 & 0.57 & 2.62 & 0.87 \\
Yellow-poplar & 0.68 & 0.62 & 2.47 & 0.83 \\
\hline \hline
\end{tabular}

$\mathbf{n} \rightarrow$ the power in the Modified Hankinson formula

In the next step, the applicable models described in section 4.1.1, including the orthotropic tensor theory and the two combination models, based on the quadratic formula and the modified Hankinson's equation, were evaluated for the accuracy of their estimation. The necessary input data $\left(\tau_{R L} ; \tau_{T L} ; \tau_{R T} ; \tau_{T R} ; \tau_{90^{\circ}}^{45^{\circ}}\right)$ were the average measured strength values. The power $(n)$ for the modified Hankinson's equation was determined by curve fitting, using the entire experimental database. Each species had its $n$ value, which is listed in Table 6.3. The model generated strength values were plotted as orthotropy diagrams for visual evaluation (Figures 6.1 to 6.3).

Due to the deficiency of complete randomization and the small sample size, conservative statistical fitting procedures resulted in lack of fit for all of the cases. Thus, $\mathrm{r}^{2}$ analysis was used to evaluate and rank the performance of the fitted models. The coefficient of determination $\left(r^{2}\right)$ is a measure of how well the model describes the data. Larger values, close to 1 , indicate that the model describes the relationship between independent and dependent variables well. The value of $r^{2}$, by definition, equals one minus the proportion of variability unexplained by the model (Dowdy and Wearden 1991). Numerically it is given by the following equation: 


$$
\mathrm{r}^{2}=1-\frac{V_{U}}{V_{T}}=1-\frac{\frac{\sum\left(\tau_{\varphi i}^{\theta}-\hat{\tau}_{\varphi}^{\theta}\right)^{2}}{N-1}}{\frac{\sum\left(\tau_{\varphi i}^{\theta}-\bar{\tau}\right)^{2}}{N-1}}=1-\frac{\sum\left(\tau_{\varphi i}^{\theta}-\hat{\tau}_{\varphi}^{\theta}\right)^{2}}{\sum\left(\tau_{\varphi i}^{\theta}-\bar{\tau}\right)^{2}}=1-\frac{S S_{E}}{S S_{T}}
$$

where: $V_{T}, V_{U} \quad$ - total variance and variance unexplained by the model;

$N \quad-$ the total number of shear strength measurements on the given species;

$\tau_{\varphi i}^{\theta} \quad-$ the $i^{\text {th }}$ measurement at grain angle $\varphi$ and ring angle $\theta$;

$\bar{\tau} \quad$ - average of the $N$ measurement points (grand average);

$\hat{\tau}_{\varphi}^{\theta} \quad$ - predicted shear strength at grain angle $\varphi$ and ring angle $\theta$;

$S S_{T}, S S_{E}$ - total sum of square of the data, and the error sum of square associated by the model.

Table 6.3 compiles the results of the coefficient of determination analyses by species and model types. For all species, the combination model based on the modified Hankinson's equation resulted in the closest agreement with experimental data. The good performance of this model was expected because the power determination was based on the entire experimental data set. Furthermore, only this model can mathematically estimate the peak stresses at small grain angle deviations.

Calculated $r^{2}$ values indicate that Equation 4.1, derived from a 4-dimensional tensor analysis, can predict the orthotropy of shear strength reasonably well. The consistency of this model regarding the quality of the predictions and its strong theoretical background encourage its use, although the model can not predict the peek stresses other than at $0^{\circ}$ grain orientation.

Conversely, the combined model, using the quadratic formula, provided poor fit to the experimental data. Although the calculated $r^{2}$ values were over 0.55 , compared to the other models, the accuracy of the predictions was significantly lower. Results imply 
that the derived equation may not be valid in all the oblique directions other than the principal anatomical planes because of the unique composite structure of solid wood.

\subsubsection{Orthotropy of compression strength and elasticity}

Tables 6.4 and 6.5 contain the summary statistics of the measured properties. These results represent all the experimental data. No outlier was discarded. The high degree of orthotropy was evident for all species for both strength and elasticity as a response to grain angle changes. However, the effect of ring orientation was not so clear. It does appear that the changing ring orientation at grain angles $<45^{\circ}$ causes strength and stiffness decrease, but at more sloping grains, the increasing ring angle tends to improve the compression properties slightly. For all species and for both properties, two-way ANOVA procedures revealed the statistically significant effect of both ring and grain angle at $95 \%$ confidence level. Furthermore, the interactions between these factors were also significant. (APPENDIX B contains the ANOVA tables for all species.) Figure 6.4 a demonstrates the stress-strain behavior of traditional compression-parallel-to-the-grain specimens by species. On Figure 6.4 b the characteristics of compression perpendicular to the grain at $15^{\circ}$ ring orientation can be seen. The long horizontal part of the diagram is the result of subsequent cellular collapse of early- then late-wood layers. The stabilized stresses in this region were considered as strength values if the specimen did not fail in shear prior to densification. Figure 6.4 c illustrates the compression behavior of yellowpoplar at different grain angles. 
Table 6.4 - Summary and basic statistics of the experimentally determined compression strength values

\section{ASPEN}

\begin{tabular}{|c|c|c|c|c|c|c|c|c|c|c|c|c|c|c|c|c|c|c|c|c|c|}
\hline \multirow{4}{*}{$\begin{array}{c}\text { Grain } \\
\text { angle } \\
\left(\varphi^{\circ}\right)\end{array}$} & \multicolumn{21}{|c|}{ Ring Orientation $\left(\theta^{\circ}\right)$} \\
\hline & \multicolumn{3}{|c|}{$\mathbf{0}$} & \multicolumn{3}{|c|}{15} & \multirow{2}{*}{\multicolumn{2}{|c|}{30}} & \multicolumn{4}{|c|}{45} & \multicolumn{3}{|c|}{60} & \multicolumn{3}{|c|}{75} & \multicolumn{3}{|c|}{90} \\
\hline & \multicolumn{19}{|c|}{ Shear Strength } & & \\
\hline & $\mathbf{N}^{\mathbf{a}}$ & $\bar{\sigma}^{b}$ & $\mathbf{s}^{\mathrm{c}}$ & $\mathbf{N}$ & $\bar{\sigma}$ & $\mathbf{S}$ & $\mathbf{N}$ & $\bar{\sigma}$ & $\mathbf{S}$ & $\mathbf{N}$ & $\bar{\sigma}$ & $\mathbf{s}$ & $\mathbf{N}$ & $\bar{\sigma}$ & $\mathbf{s}$ & $\mathbf{N}$ & $\bar{\sigma}$ & $\mathbf{S}$ & $\mathbf{N}$ & $\bar{\sigma}$ & $\mathbf{S}$ \\
\hline 0 & - & - & - & - & - & - & - & - & - & 10 & 36.23 & 1.14 & - & - & - & - & - & - & - & - & - \\
\hline 15 & - & - & - & - & - & - & - & - & - & 10 & 28.55 & 2.64 & - & - & - & - & - & - & - & - & - \\
\hline 30 & 6 & 10.54 & 1.99 & 4 & 11.33 & 0.33 & 4 & 11.05 & 0.44 & 6 & 12.27 & 1.21 & 4 & 13.25 & 1.17 & 4 & 10.54 & 4.94 & 6 & 12.55 & 0.71 \\
\hline 45 & 6 & 6.52 & 0.52 & 4 & 8.15 & 1.12 & 4 & 6.09 & 0.60 & 6 & 5.13 & 0.39 & 4 & 6.61 & 0.78 & 4 & 6.16 & 0.19 & 6 & 8.35 & 0.89 \\
\hline 60 & 6 & 3.63 & 0.19 & 4 & 4.24 & 0.19 & 4 & 4.05 & 0.54 & 6 & 3.88 & 0.37 & 4 & 3.77 & 0.02 & 4 & 5.81 & 0.08 & 6 & 5.08 & 1.24 \\
\hline 75 & 6 & 3.18 & 0.30 & 4 & 2.88 & 0.12 & 4 & 3.01 & 0.13 & 6 & 2.62 & 0.10 & 4 & 3.31 & 0.60 & 4 & 4.97 & 0.14 & 6 & 3.12 & 0.10 \\
\hline 90 & 6 & 3.07 & 0.13 & 4 & 3.07 & 0.18 & 4 & 3.38 & 0.13 & 6 & 3.44 & 0.09 & 4 & 3.70 & 0.24 & 4 & 4.14 & 0.10 & 5 & 4.28 & 0.13 \\
\hline
\end{tabular}

\section{OAK}

\begin{tabular}{|c|c|c|c|c|c|c|c|c|c|c|c|c|c|c|c|c|c|c|c|c|c|}
\hline \multirow{4}{*}{$\begin{array}{c}\text { Grain } \\
\text { angle } \\
\left(\varphi^{\circ}\right)\end{array}$} & \multicolumn{21}{|c|}{ Ring Orientation $\left(\theta^{\circ}\right)$} \\
\hline & \multicolumn{3}{|c|}{$\mathbf{0}$} & \multicolumn{3}{|c|}{15} & \multicolumn{2}{|r|}{30} & \multicolumn{3}{|c|}{45} & \multicolumn{4}{|c|}{60} & \multicolumn{3}{|c|}{75} & \multicolumn{3}{|c|}{90} \\
\hline & \multicolumn{21}{|c|}{ Shear Strength } \\
\hline & $\mathbf{N}$ & $\bar{\sigma}$ & S & $\mathbf{N}$ & $\bar{\sigma}$ & $\mathbf{s}$ & $\mathbf{N}$ & $\bar{\sigma}$ & $\mathbf{s}$ & $\mathbf{N}$ & $\bar{\sigma}$ & $\mathbf{S}$ & ' & $\bar{\sigma}$ & s & $\mathbf{N}$ & $\bar{\sigma}$ & $\mathbf{S}$ & $\mathbf{N}$ & $\bar{\sigma}$ & s \\
\hline 0 & - & - & - & - & - & - & - & - & - & 9 & 49.72 & 2.75 & - & - & - & - & - & - & - & - & - \\
\hline 15 & - & - & - & - & - & - & - & - & - & 10 & 41.76 & 2.67 & - & - & - & - & - & - & - & - & - \\
\hline 30 & 6 & 28.31 & 1.58 & 4 & 33.23 & 1.95 & 4 & 26.81 & 1.74 & 6 & 18.17 & 5.08 & 4 & 27.74 & 2.00 & 4 & 25.72 & 1.62 & 6 & 24.25 & 1.11 \\
\hline 45 & 6 & 22.13 & 0.34 & 4 & 23.24 & 1.94 & 4 & 17.18 & 0.69 & 6 & 13.14 & 0.14 & 4 & 16.96 & 2.77 & 4 & 14.42 & 0.31 & 6 & 17.01 & 0.58 \\
\hline 60 & 6 & 13.91 & 0.54 & 4 & 16.06 & 0.75 & 4 & 12.99 & 0.99 & 6 & 13.62 & 1.70 & 4 & 10.85 & 0.64 & 4 & 10.23 & 0.05 & 6 & 13.07 & 0.60 \\
\hline 75 & 6 & 11.25 & 0.21 & 4 & 13.30 & 0.41 & 4 & 9.81 & 0.30 & 6 & 11.03 & 0.20 & 4 & 9.19 & 0.52 & 4 & 8.22 & 0.13 & 6 & 11.29 & 0.35 \\
\hline 90 & 6 & 10.17 & 0.21 & 3 & 11.63 & 0.42 & 4 & 9.35 & 0.24 & 6 & 9.77 & 0.49 & 4 & 9.36 & 0.17 & 4 & 7.65 & 0.29 & 6 & 11.32 & 0.43 \\
\hline
\end{tabular}

\section{YELLOW-POPLAR}

\begin{tabular}{|c|c|c|c|c|c|c|c|c|c|c|c|c|c|c|c|c|c|c|c|c|c|}
\hline \multirow{4}{*}{$\begin{array}{c}\text { Grain } \\
\text { angle } \\
\left(\varphi^{\circ}\right)\end{array}$} & \multicolumn{21}{|c|}{ Ring Orientation $\left(\theta^{\circ}\right)$} \\
\hline & \multicolumn{3}{|c|}{$\mathbf{0}$} & \multicolumn{3}{|c|}{15} & \multirow{2}{*}{\multicolumn{2}{|c|}{30}} & \multicolumn{3}{|c|}{45} & \multicolumn{4}{|c|}{60} & \multicolumn{3}{|c|}{75} & \multicolumn{3}{|c|}{90} \\
\hline & \multicolumn{20}{|c|}{ Shear Strength } & \\
\hline & $\mathbf{N}$ & $\bar{\sigma}$ & s & $\mathbf{N}$ & $\bar{\sigma}$ & $\mathbf{s}$ & $\mathbf{N}$ & $\bar{\sigma}$ & $\mathbf{S}$ & $\mathbf{N}$ & $\bar{\sigma}$ & $\mathbf{S}$ & ' $\mathbf{N}$ & $\bar{\sigma}$ & $\mathbf{s}$ & $\mathbf{N}$ & $\bar{\sigma}$ & $\mathbf{s}$ & $\mathbf{N}$ & $\bar{\sigma}$ & s \\
\hline 0 & - & - & - & - & - & - & - & - & - & 10 & 35.47 & 2.52 & - & - & - & - & - & - & - & - & - \\
\hline 15 & - & - & - & - & - & - & - & - & - & 10 & 33.35 & 3.74 & - & - & - & - & - & - & - & - & - \\
\hline 30 & 6 & 20.84 & 1.44 & 4 & 19.03 & 0.40 & 4 & 21.37 & 0.87 & 6 & 19.55 & 0.97 & 4 & 16.70 & 1.20 & 4 & 15.56 & 1.07 & 6 & 17.33 & 0.41 \\
\hline 45 & 6 & 13.08 & 0.68 & 4 & 12.89 & 4.32 & 4 & 12.80 & 0.42 & 6 & 13.77 & 0.88 & 4 & 11.46 & 0.20 & 4 & 9.07 & 0.18 & 6 & 9.22 & 1.76 \\
\hline 60 & 6 & 8.19 & 0.09 & 4 & 7.97 & 0.11 & 4 & 8.40 & 0.29 & 6 & 7.81 & 0.29 & 4 & 8.07 & 0.18 & 4 & 6.66 & 0.24 & 6 & 8.12 & 0.22 \\
\hline 75 & 6 & 6.71 & 0.19 & 4 & 6.50 & 0.06 & 4 & 6.80 & 0.14 & 6 & 5.46 & 0.37 & 4 & 6.35 & 0.21 & 4 & 5.60 & 0.14 & 6 & 5.35 & 0.28 \\
\hline 90 & 6 & 4.38 & 0.15 & 4 & 5.99 & 0.14 & 4 & 6.31 & 0.08 & 6 & 4.42 & 0.08 & 4 & 6.40 & 0.23 & 4 & 5.16 & 0.10 & 6 & 7.13 & 0.16 \\
\hline
\end{tabular}

a - sample size

$\mathrm{b}-$ mean compression strength value $(\mathrm{MPa})$

$\mathrm{c}-$ standard deviation $(\mathrm{MPa})$ 
Table 6.5 - Summary and basic statistics of the experimentally determined compression MOE values

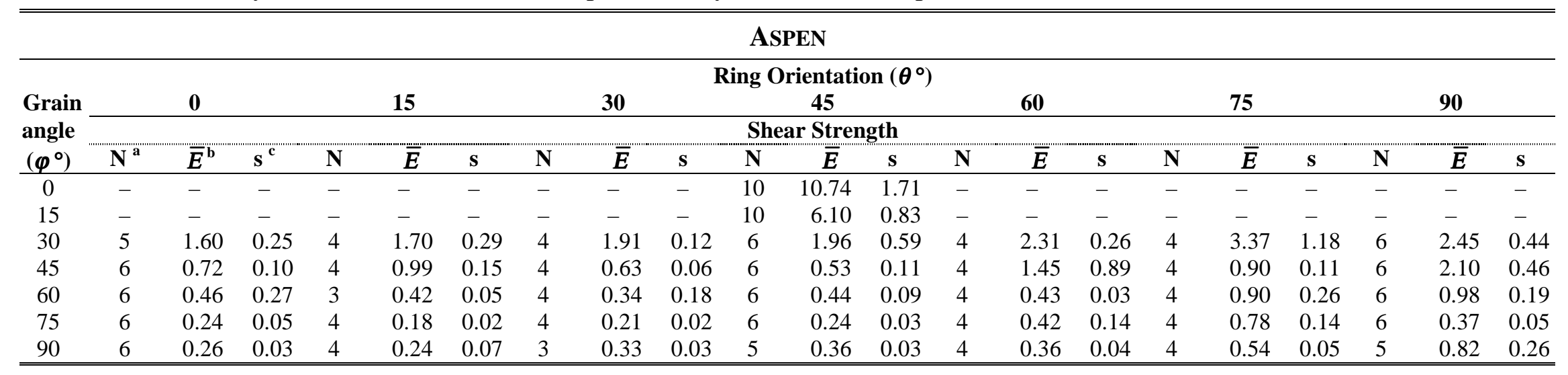

\section{OAK}

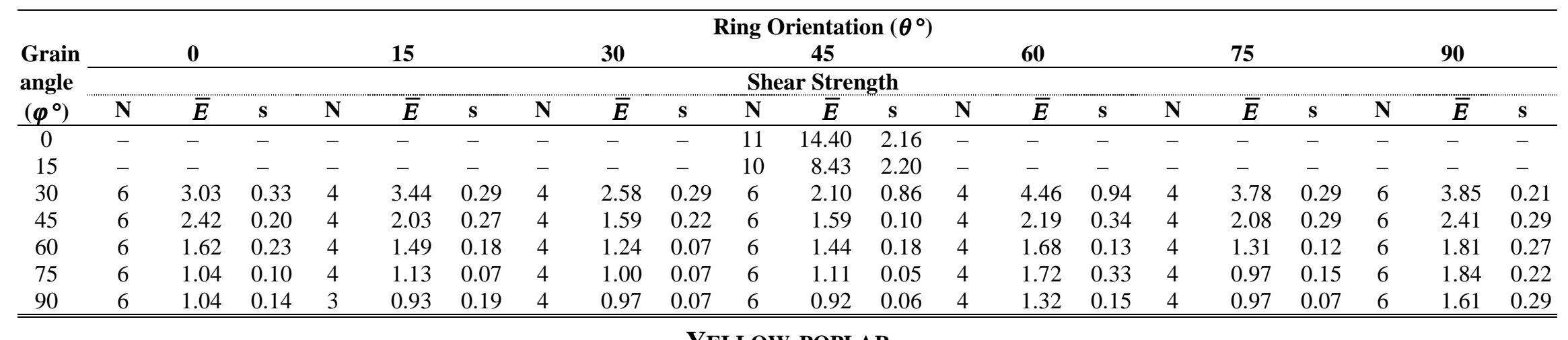

\section{YELLOW-POPLAR}

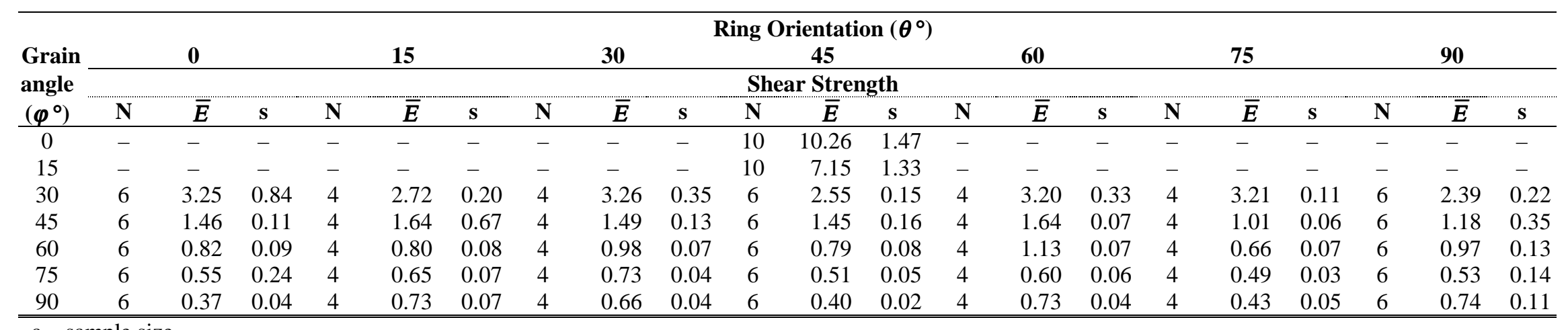

a - sample size

$\mathrm{b}$ - mean compression MOE value $(\mathrm{GPa})$

$\mathrm{c}$ - standard deviation (GPa) 


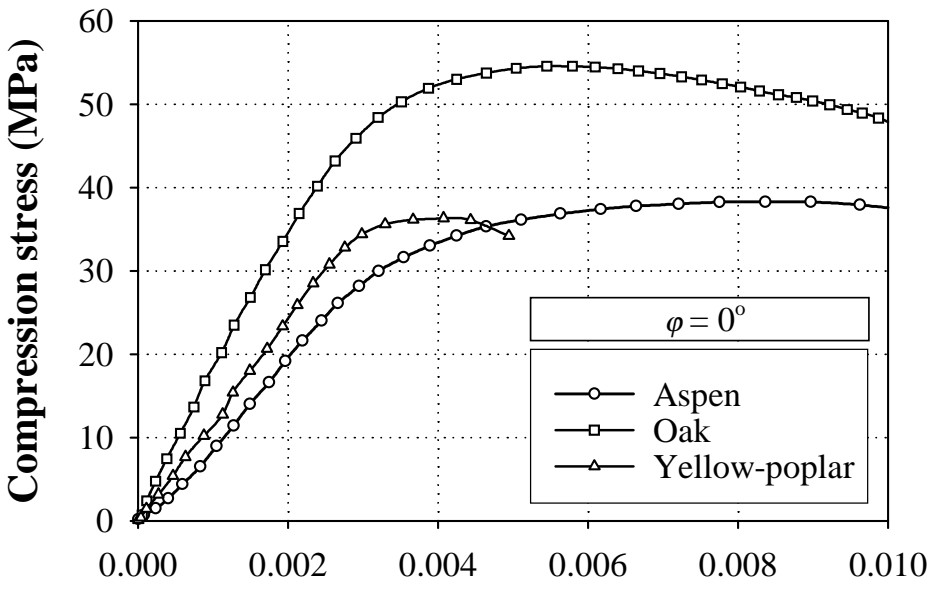

a.

\section{Strain}

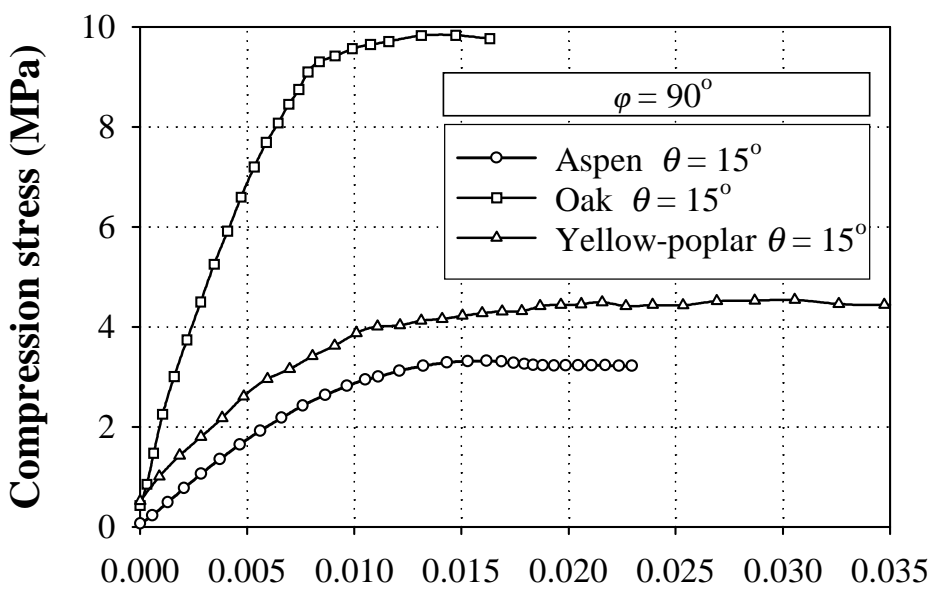

b.

\section{Strain}

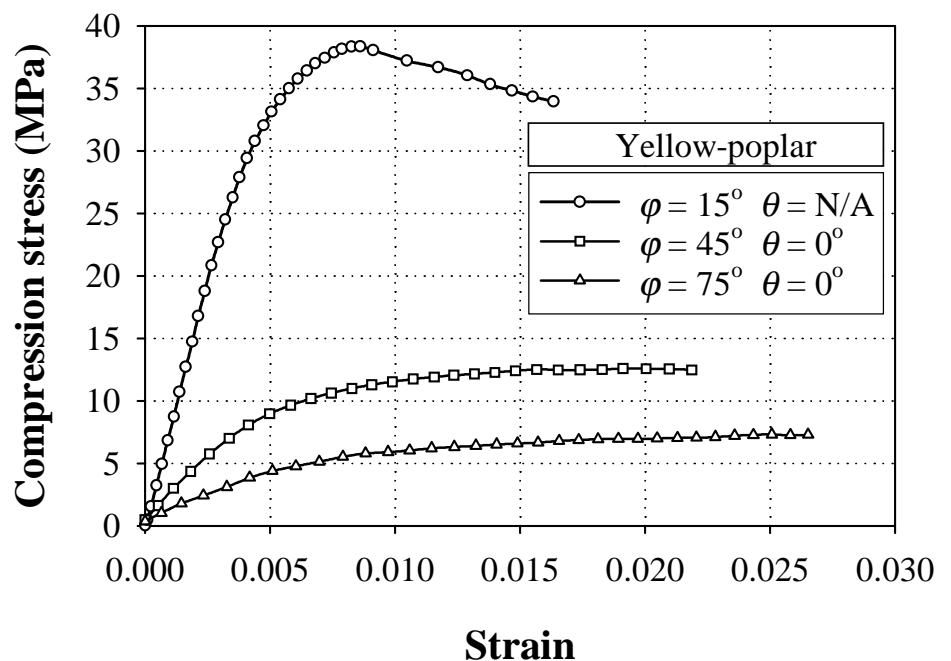

Figure 6.4 - Typical stress-strain diagrams

a. Traditional, parallel to the grain compression b. Perpendicular to the grain compression c. The effect of grain orientation 


\subsubsection{Prediction of the compression properties}

The mean values were used to create anisotropy diagrams in a three dimensional polar coordinate system. Such diagrams are shown on Figures 6.5 a and 6.5 d for strength and MOE of aspen, respectively. The seven values, indicated at $15^{\circ}$ grain angle, all correspond to the one average value of ten replications that were measured at this level at random ring orientations. The intermediate mesh data were interpolated using the inverse distance method (SPSS Inc. 1997). Figures 6.6 and 6.7 contain similar diagrams for red oak and yellow-poplar, respectively.

In the next step, the two models discussed in section 4.1.2.2 were evaluated for the accuracy of their estimation. The required model inputs, were the average measured strength and MOE values. The model-generated property data were plotted as orthotropy diagrams for visual evaluation. Figures 6.5 through 6.7 contains these diagrams for aspen, read oak and yellow-poplar. Diagrams marked by $\mathbf{b}$ and $\mathbf{e}$ represent the strength and MOE values predicted by Equation 4.10, derived from tensor analysis. The $\mathbf{c}$ and $\mathbf{f}$ details show the predictions of three-dimensional Hankinson's formula. The diagrams clearly confirm the individual and interaction effect of $\varphi$ and $\theta$ on the strength and MOE in compression.

Statistical comparisons of experimental and predicted properties by $r^{2}$ analyses (Equation 6.1) confirmed the applicability of these models. Table $\mathbf{6 . 6}$ contains the results of the analyses by species and model types. For aspen and red oak compression strength and MOE, both models guaranteed almost equally excellent agreement $\left(r^{2}>0.9\right)$. 


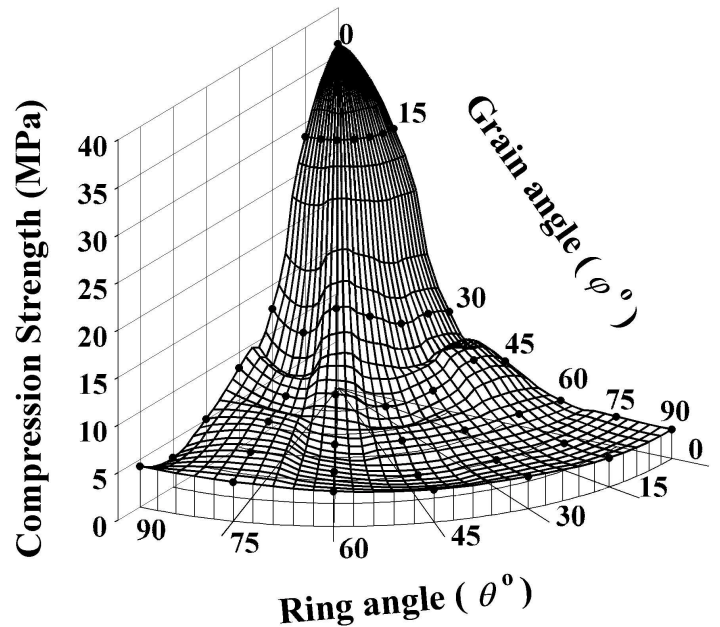

a. - Experimental strength values

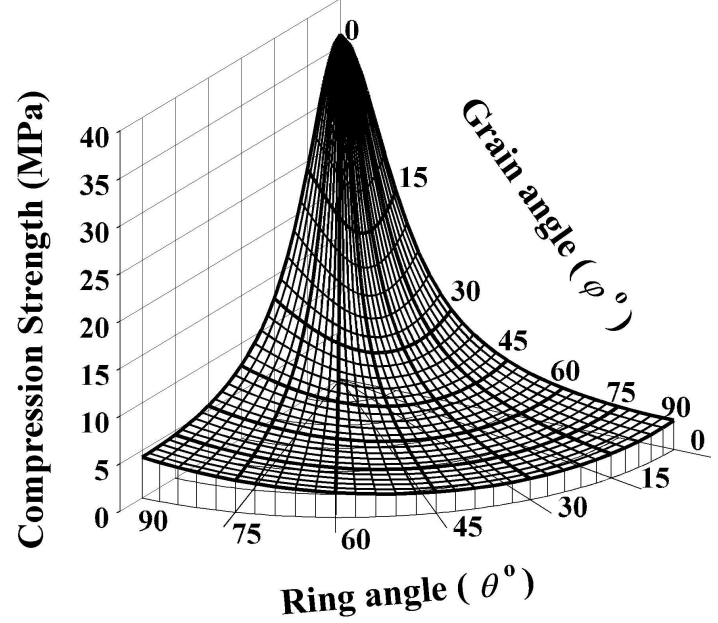

b. - Predicted -3 D Hankinson formula

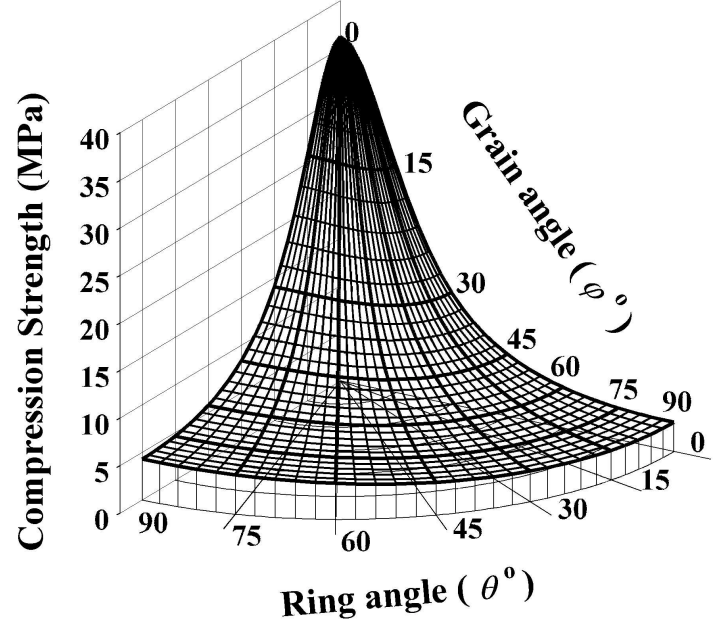

C. - Predicted - Orthotropic tensor theory

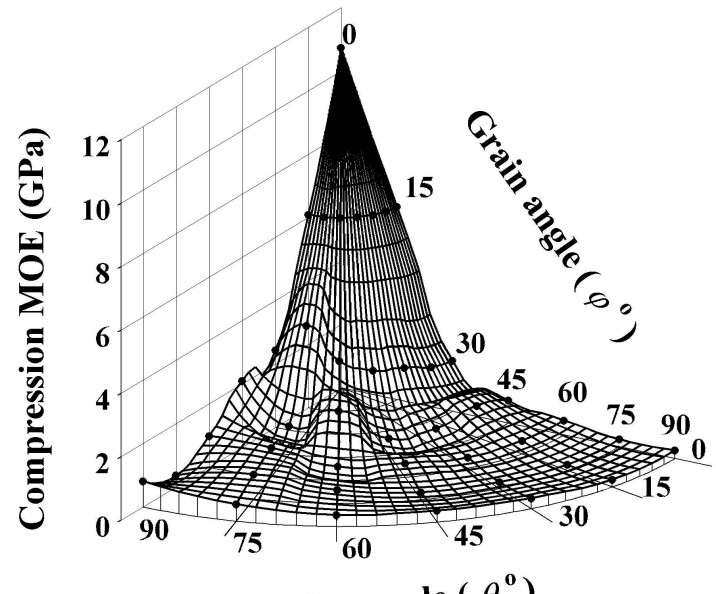

Ring angle $\left(\theta^{0}\right)$

d. - Experimental MOE values

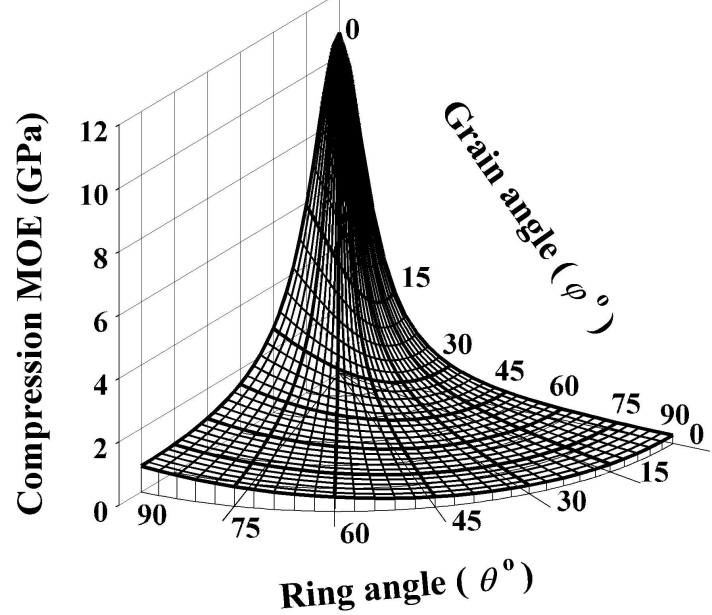

e. - Predicted - 3D Hankinson formula

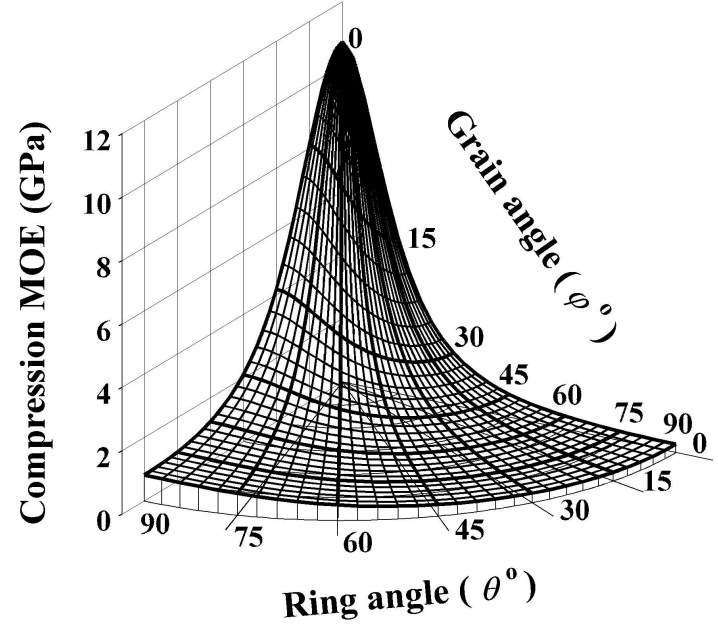

f. - Predicted - Orthotropic tensor theory

Figure 6.5 - Orthotropic diagrams of compression strength and elasticity - quaking aspen 


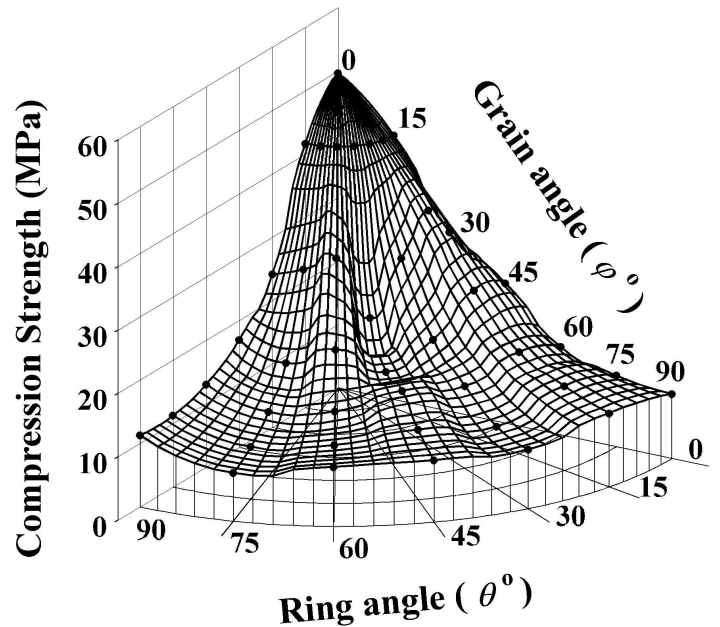

a. - Experimental strength

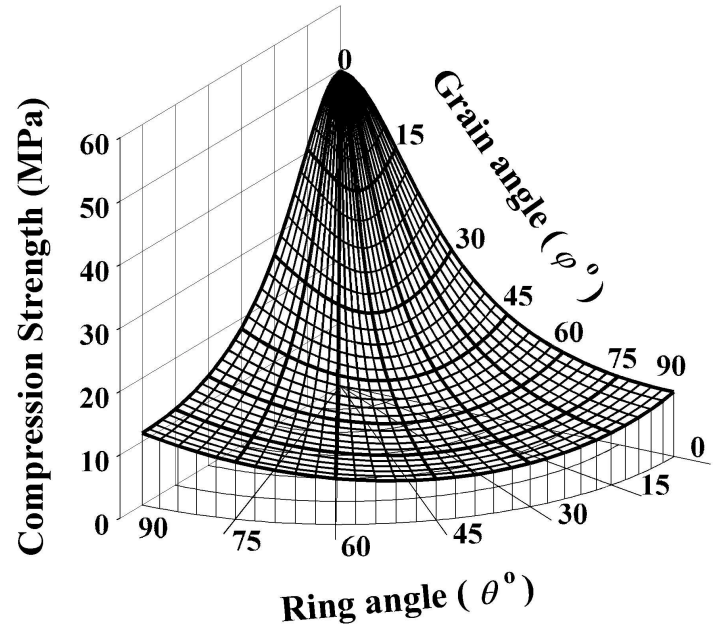

b. - Predicted - 3D Hankinson

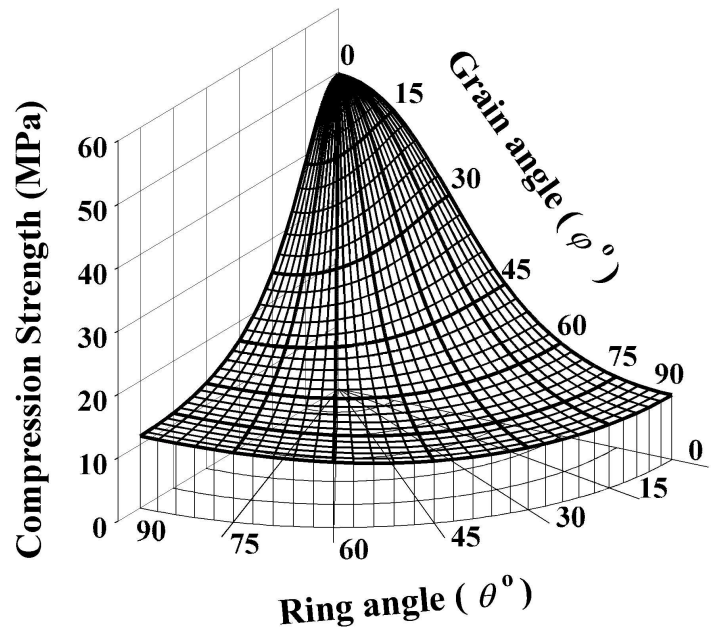

C. - Predicted - Orthotropic tensor

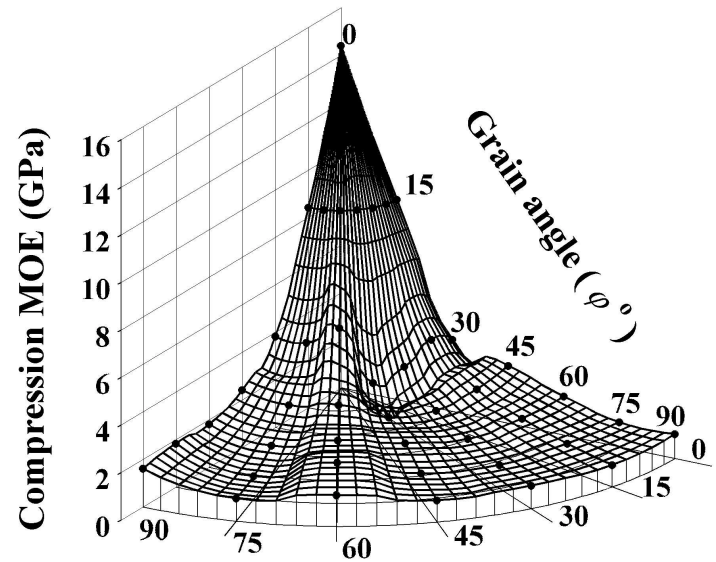

Ring angle $\left(\theta^{0}\right)$

d. - Experimental MOE

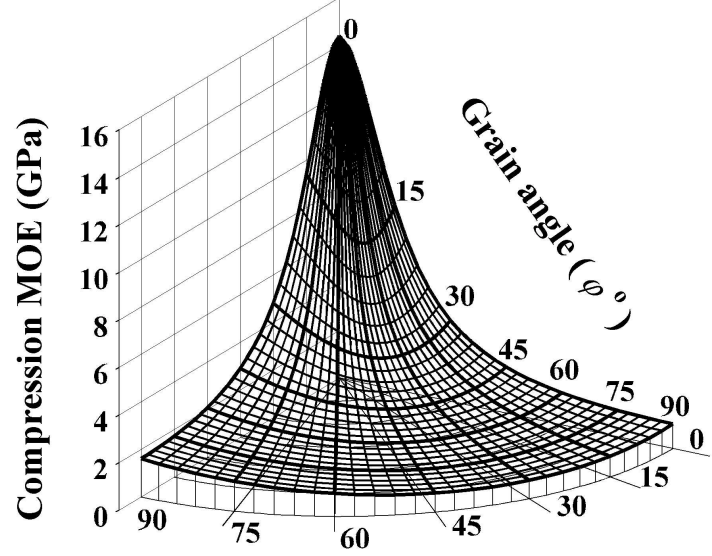

Ring angle $\left(\theta^{0}\right)$

e. - Predicted - 3D Hankinson

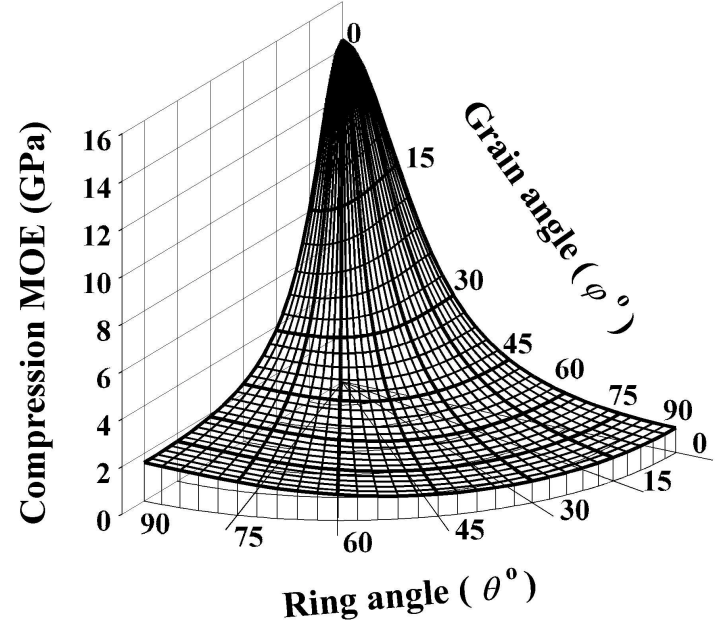

f. - Predicted - Orthotropic tensor

Figure 6.6 - Orthotropic diagrams of compression strength and elasticity - red oak 


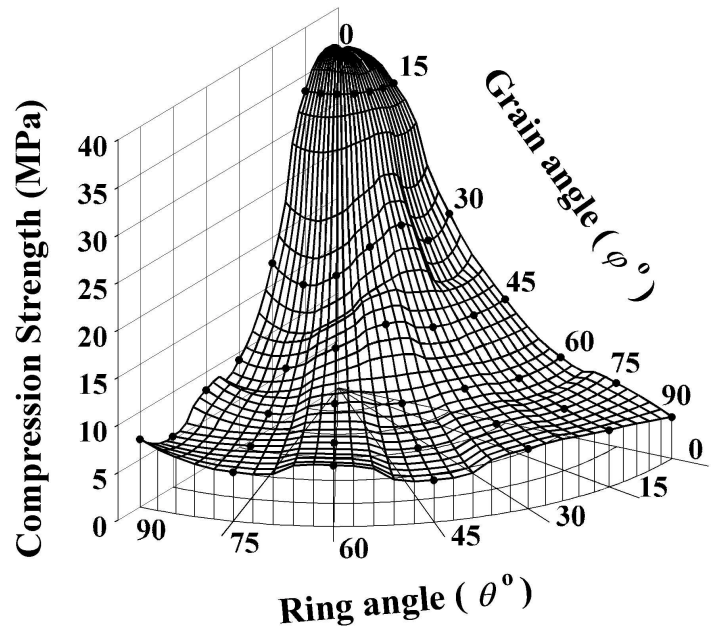

a. - Experimental strength

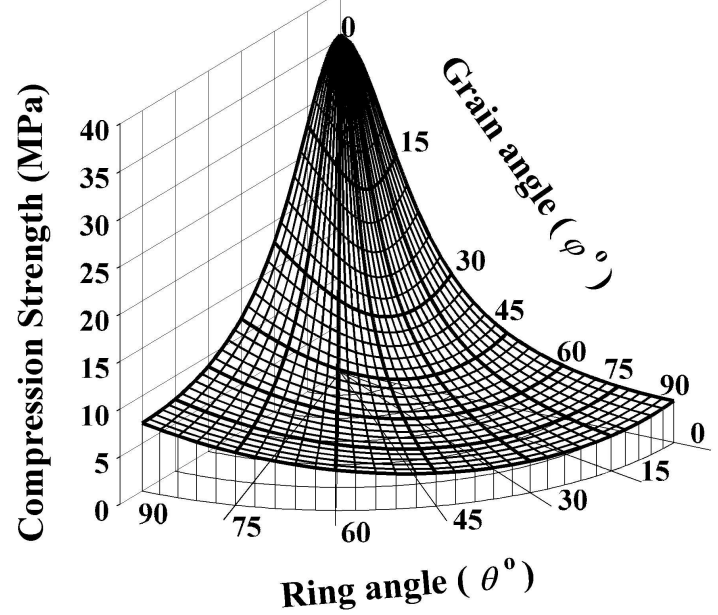

b. - Predicted - 3D Hankinson

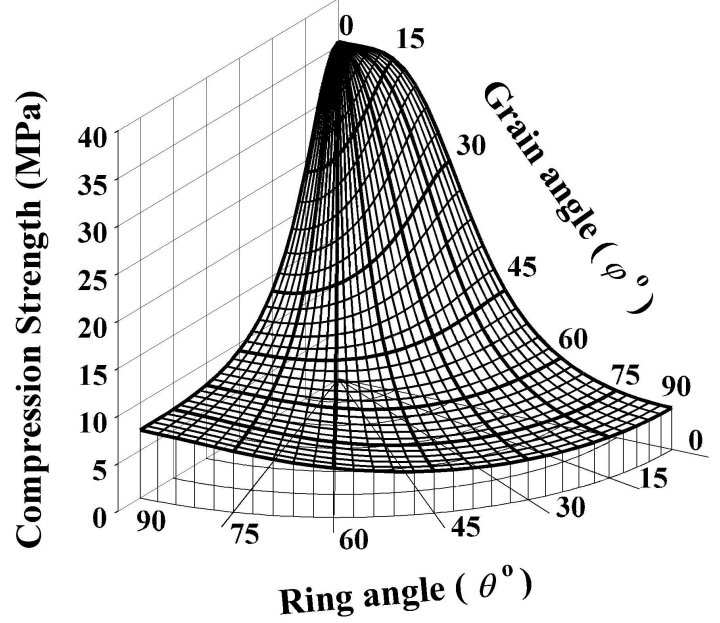

C. - Predicted - Orthotropic tensor

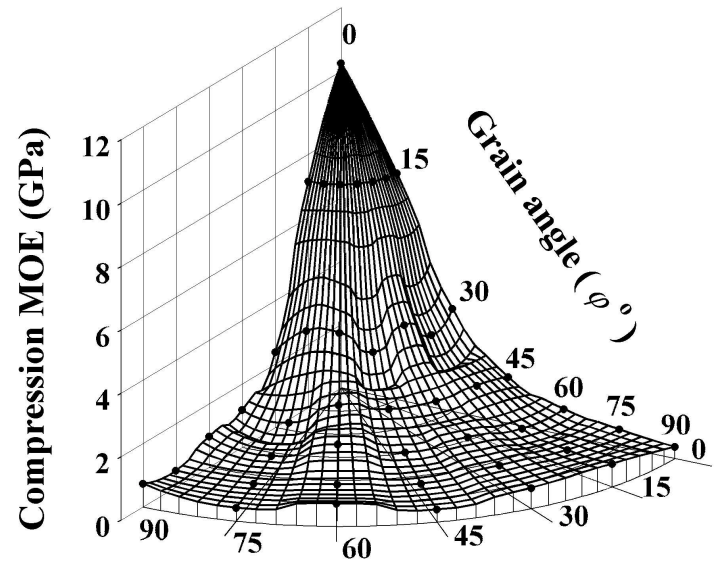

Ring angle $\left(\theta^{0}\right)$

d. - Experimental MOE

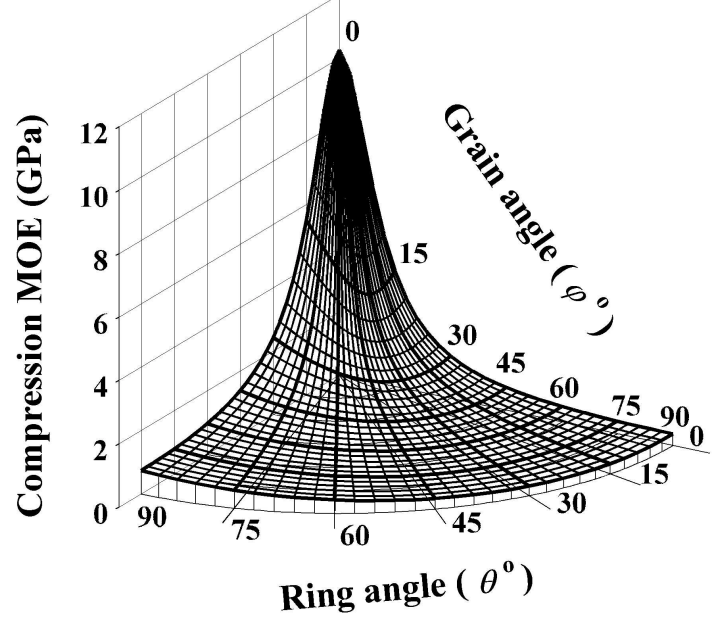

e. - Predicted - 3D Hankinson

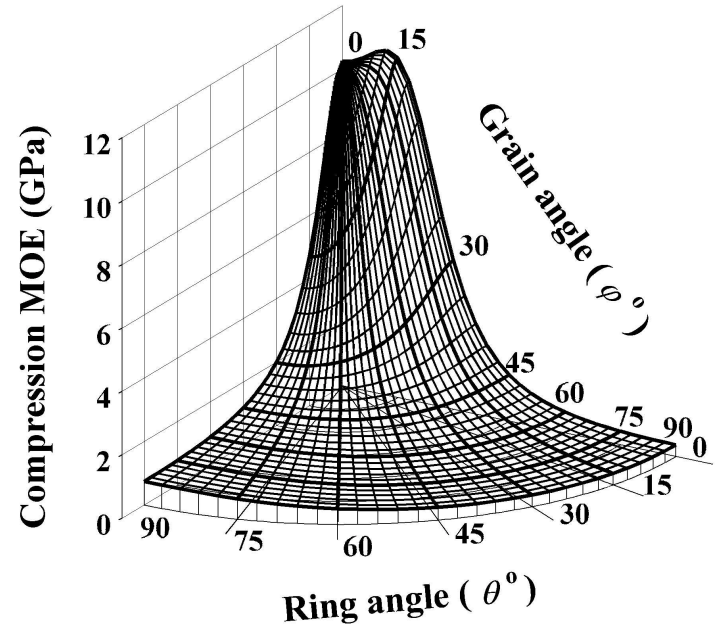

f. - Predicted - Orthotropic tensor

Figure 6.7 - Orthotropic diagrams of compression strength and elasticity - yellow-poplar 
Table 6.6. - Coefficients of determination provided by the two prediction models for compression strength and MOE

\begin{tabular}{lcccc}
\hline \hline \multicolumn{1}{c}{ Species } & $\begin{array}{c}\text { Coefficient of determination } \\
\text { Orthotropic tensor theory }\end{array}$ & \multicolumn{2}{c}{$\begin{array}{c}\text { Coefficient of determination } \\
\text { 3-D Hankinson formula }\end{array}$} \\
& $\begin{array}{c}\text { Compression } \\
\text { strength }\end{array}$ & $\begin{array}{c}\text { Compression } \\
\text { MOE }\end{array}$ & $\begin{array}{c}\text { Compression } \\
\text { strength }\end{array}$ & $\begin{array}{c}\text { Compression } \\
\text { MOE }\end{array}$ \\
\hline Aspen & 0.93 & 0.94 & 0.91 & 0.91 \\
Red oak & 0.93 & 0.93 & 0.93 & 0.94 \\
Yellow-poplar & 0.92 & 0.93 & 0.72 & 0.83 \\
\hline \hline
\end{tabular}

For yellow-poplar, however, the prediction quality of the Hankinson's formula decreased, although the $r^{2}$ values remained on acceptable levels for both strength and stiffness. The phenomena may be explained by the sensitivity of Hankinson's equation to small grain angle variations. This is particularly manifested during strength predictions where the effect of small grain deviation on the experimentally measured strength is less significant.

\subsubsection{Failure mode analysis}

During the compression strength determination, several types of failure were observed. Figure 6.8 shows the most characteristic failure modes, where specimen $a$ demonstrates a typical compression failure. Specimens $b$ and $c$ failed as a combination of cellular collapse and shear as indicated by the horizontal dislocation of certain parts of the blocks. Clear shear failures in the $L R$ an $L T$ planes are represented by specimens $d$ and $e$, respectively. Most of the perpendicular-to-the-grain specimens $\left(\varphi=90^{\circ}\right)$ did not fail at all, due to the infinite densification. For safety reasons, tests were disrupted as soon as the stress level explicitly stabilized in the plateau region (Figure 6.4 b). 


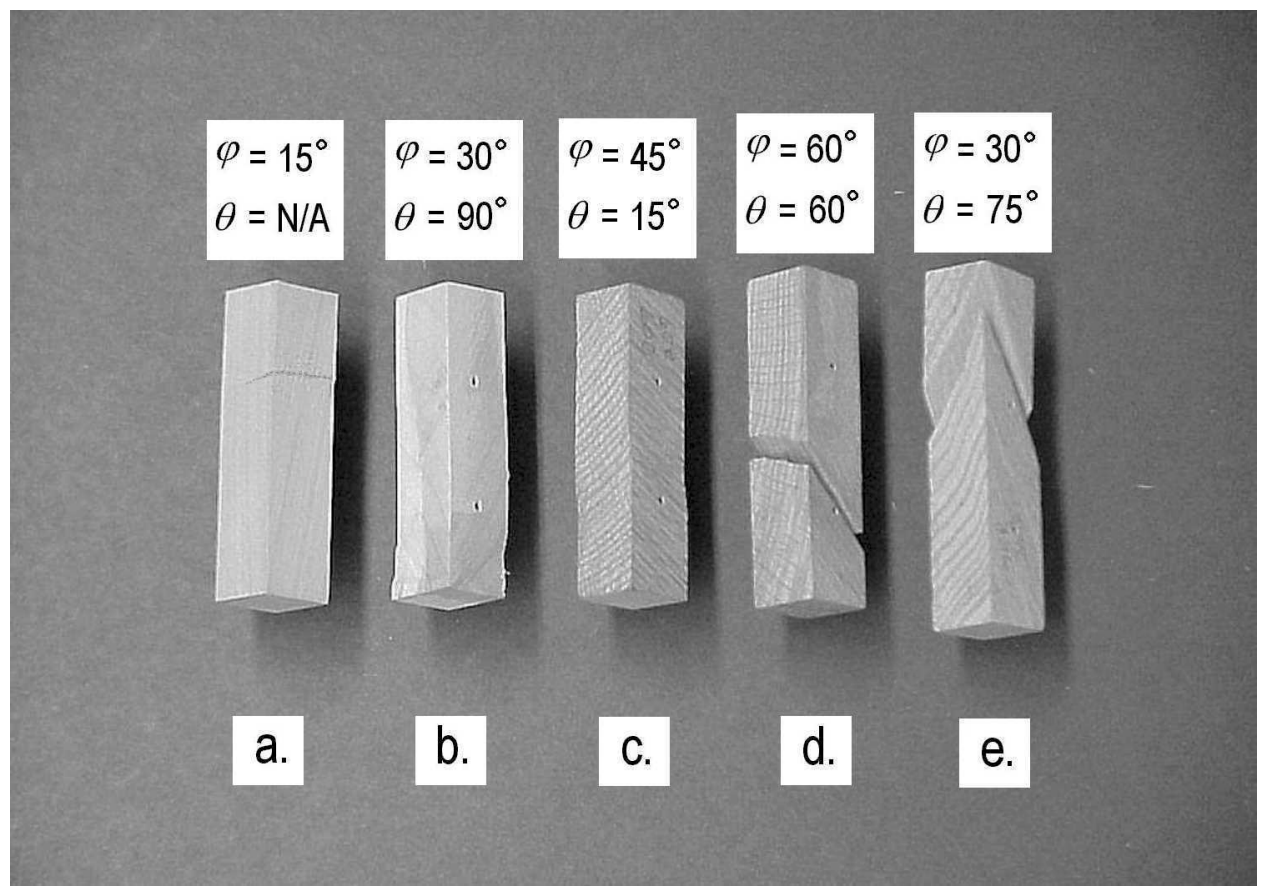

Figure 6.8 - Characteristic failure modes of compression specimens; $a$ - compression; $b, c$-combined compression and shear; $d, e-$ shear failure

The complexity of failure mode prediction has been addressed briefly in section 4.1.2.1. In further elaboration of the issue, Figure 6.9 demonstrates the likelihood of shear failure assessment for two particular angle combinations under compression stress.

First, consider a red oak specimen with $\varphi=45^{\circ}$ and $\theta=15^{\circ}$ grain and ring orientations under $\sigma_{u}=23.24 \mathrm{MPa}$ compression stress in the global coordinate system. Note that $\sigma_{u}$ is the average compression strength of oak for the particular $\varphi, \theta$ combination (Table 6.4). In Figure 6.9, the mesh represents the orthotropic shear strength of oak in planes parallel to the grain (see Figure 6.2 d). Possible combinations of $\vartheta$ and $\Theta$ are defined by the inter-dependence of the grain orientation of $F_{V}$ and the rotation angle of the parallel-to-the-grain sheared plane (see Figure 4.4.) For a detailed derivation of the 


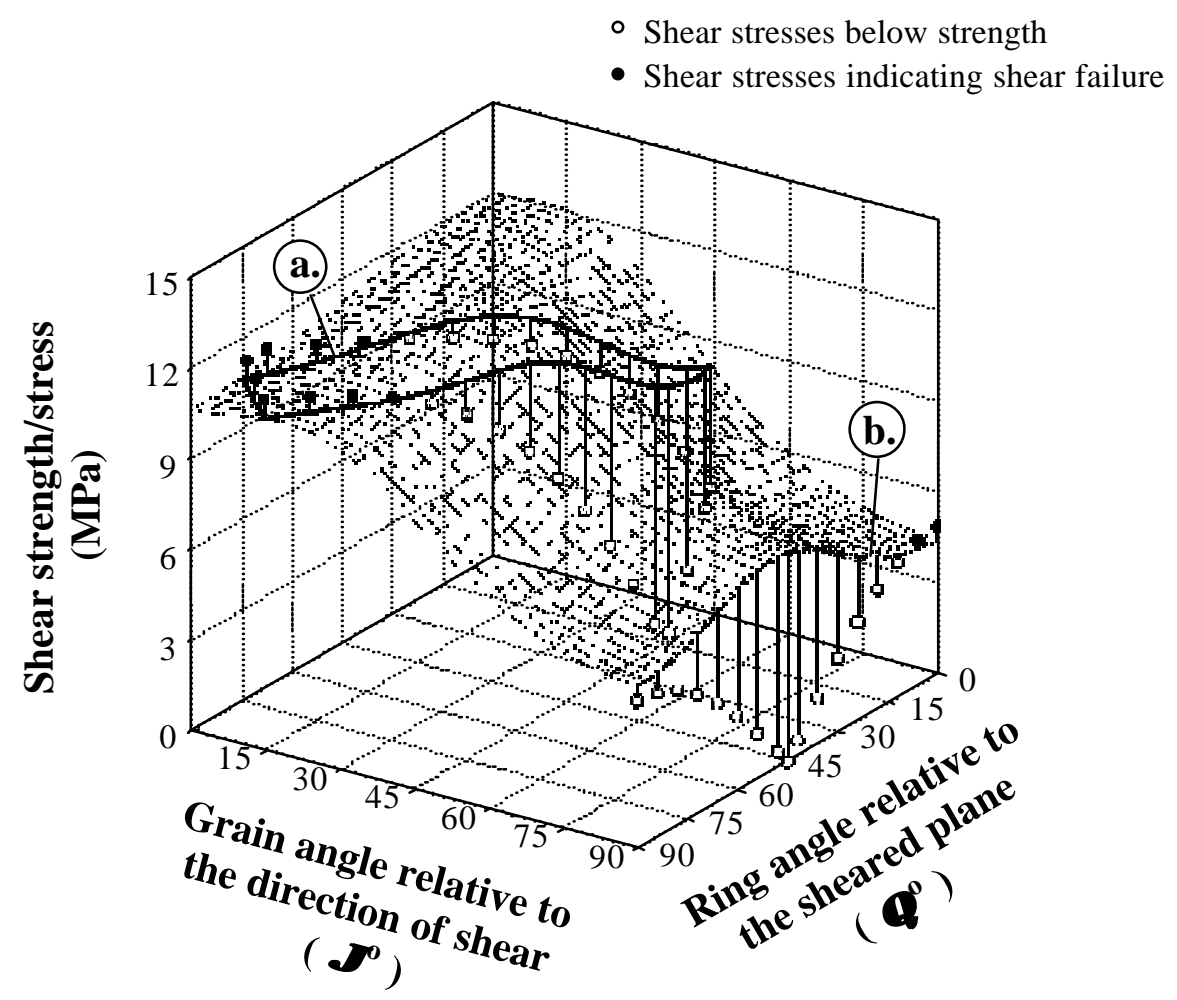

Figure 6.9 - Likelihood of shear failure in compression specimens.

a. - critical shear stress contour of specimen $\varphi=45^{\circ}, \theta=15^{\circ} ; \sigma_{u}=23.24 \mathrm{MPa}$

b. - critical shear stress contour of specimen $\varphi=90^{\circ}, \theta=45^{\circ} ; \sigma_{u}=9.77 \mathrm{MPa}$

relationships between $\varphi, \theta, \vartheta$ and $\Theta$, see APPENDIX $\boldsymbol{C}$. Shear strength values at these angle combinations form the solid line within the strength surface, which denotes the possible shearing scenarios of the specimen. This line can be considered as the critical shear stress contour of the examined $\varphi, \theta$ compression specimen. The corresponding shear stresses, computed from $\sigma_{u}=23.24 \mathrm{MPa}$ and the inclined sectional areas $(A)$, are represented by the symbols and drop-lines. Solid symbols mark the compression-induced shear stresses that exceed the estimated shear strength, while the empty symbols mark the stresses that are below the strength values. Theoretically, the specimens should have 
failed in shear. However, because of the natural variability of wood and the presence normal stresses in the sheared planes, usually a combined failure was observed as in specimen c on Figure $6.8 c$.

The second example demonstrates that compression may induce shear failure in a horizontally grained specimen too. For the $\varphi=90^{\circ}, \theta=45^{\circ}$ specimens the average compression strength $\left(\sigma_{u}\right)$ was $9.77 \mathrm{MPa}$. The critical shear stress contour reduces to a single line (dotted in Figure 6.9) along the lower edge of the shear strength surface, because the direction of shear is always perpendicular to the direction of grain for this specimen configuration. The calculated stresses exceed the strength values in the range of $15^{\circ}>\Theta>0^{\circ}$. This domain represents inclined planes around $45^{\circ}$ relative to the length of the specimen, in which shear failure may occur. At $\Theta=45^{\circ}$ the shear stress is zero because no shear can be induced along the vertical or horizontal planes in the global coordinate system. Unfortunately, the practical application of such analysis is limited, because of the uncertainties in shear strength determination and variations in growth characteristics of natural wood.

\subsubsection{Orthotropic of tensile elasticity of structural veneers}

Table 6.7 shows the summary statistics of the experimental stress-wave MOE of each species at the seven grain angle levels. Figures 6.10 a through 6.12 a contain box plots of the measured MOE values at each grain orientation. 
Table 6.7 - Summary statistics of the experimentally determined dynamic MOE values (GPa)

\begin{tabular}{|c|c|c|c|c|c|c|c|c|}
\hline & & \multicolumn{7}{|c|}{$\varphi^{\circ}$} \\
\hline & & 0 & 15 & 30 & 45 & 60 & 75 & 90 \\
\hline \multirow{4}{*}{$\begin{array}{l}\text { QUAKING ASPEN } \\
\qquad(\mathrm{n}=20)\end{array}$} & Mean & 11.976 & 7.056 & 2.658 & 1.279 & 1.048 & 1.035 & 1.030 \\
\hline & $\mathrm{s}$ & 0.888 & 0.752 & 0.287 & 0.135 & 0.045 & 0.045 & 0.047 \\
\hline & Min & 10.757 & 5.697 & 2.185 & 1.111 & 0.982 & 0.963 & 0.959 \\
\hline & Max & 13.866 & 13.866 & 8.791 & 3.239 & 1.680 & 1.163 & 1.152 \\
\hline \multirow{4}{*}{$\begin{array}{l}\text { RED OAK } \\
\qquad(\mathrm{n}=23)\end{array}$} & Mean & 10.651 & 5.770 & 1.997 & 1.396 & 1.387 & 1.383 & 1.382 \\
\hline & $\mathrm{s}$ & 1.201 & 0.827 & 0.348 & 0.046 & 0.039 & 0.039 & 0.044 \\
\hline & Min & 8.135 & 4.625 & 1.450 & 1.309 & 1.309 & 1.306 & 1.284 \\
\hline & Max & 12.508 & 8.195 & 2.811 & 1.483 & 1.461 & 1.455 & 1.462 \\
\hline \multirow{4}{*}{$\begin{array}{l}\text { YELLOW-POPLAR } \\
\qquad(\mathrm{n}=21)\end{array}$} & Mean & 13.529 & 7.631 & 2.589 & 1.254 & 1.149 & 1.145 & 1.142 \\
\hline & $\mathrm{s}$ & 1.020 & 0.735 & 0.254 & 0.112 & 0.050 & 0.060 & 0.062 \\
\hline & Min & 11.907 & 6.507 & 2.218 & 1.133 & 1.032 & 1.015 & 1.003 \\
\hline & Max & 15.496 & 9.555 & 3.347 & 1.668 & 1.206 & 1.279 & 1.254 \\
\hline
\end{tabular}




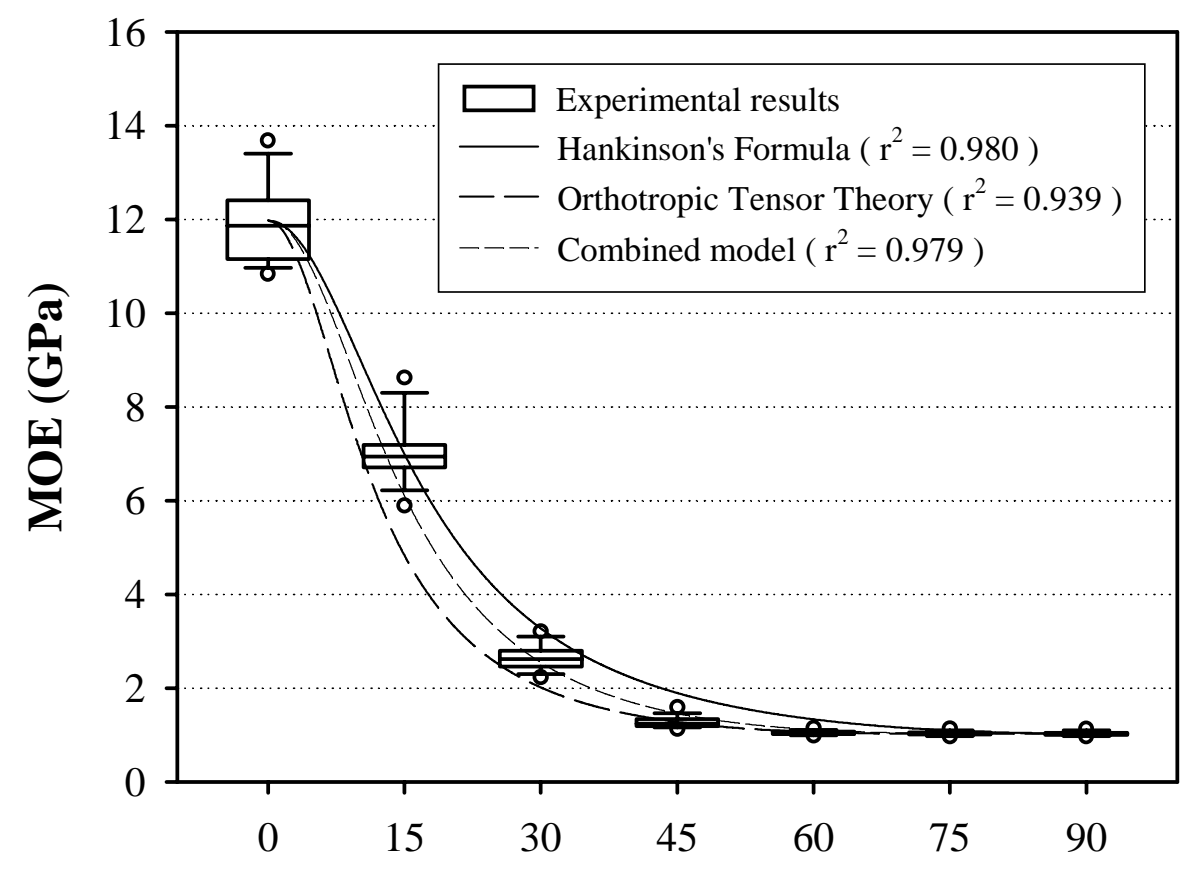

a.

Grain angle $\left(\varphi^{\circ}\right)$

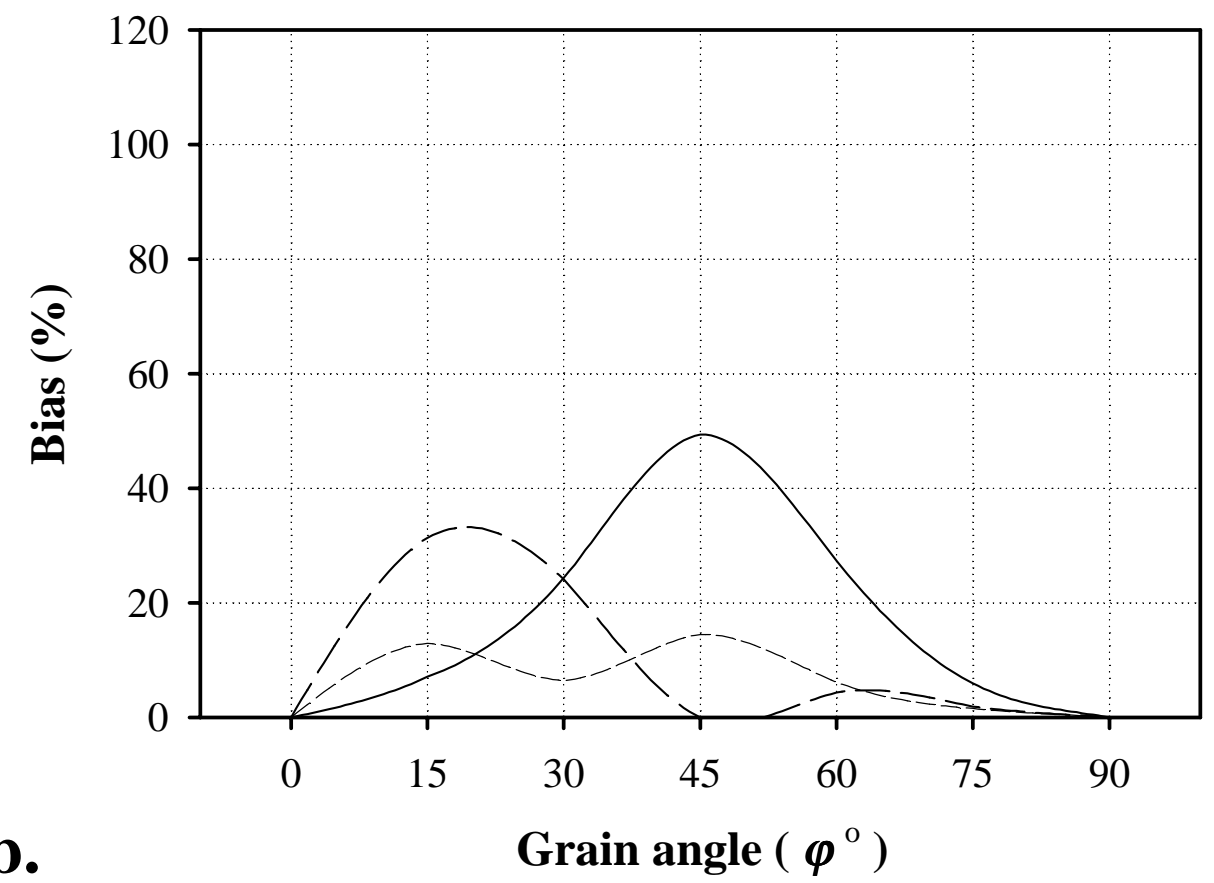

Figure 6.10 - Orthotropic dynamic MOE of quaking aspen structural veneer sheets a. - experimental and model predicted values

b. - Average Absolute Percentage Bias associated with the different models 


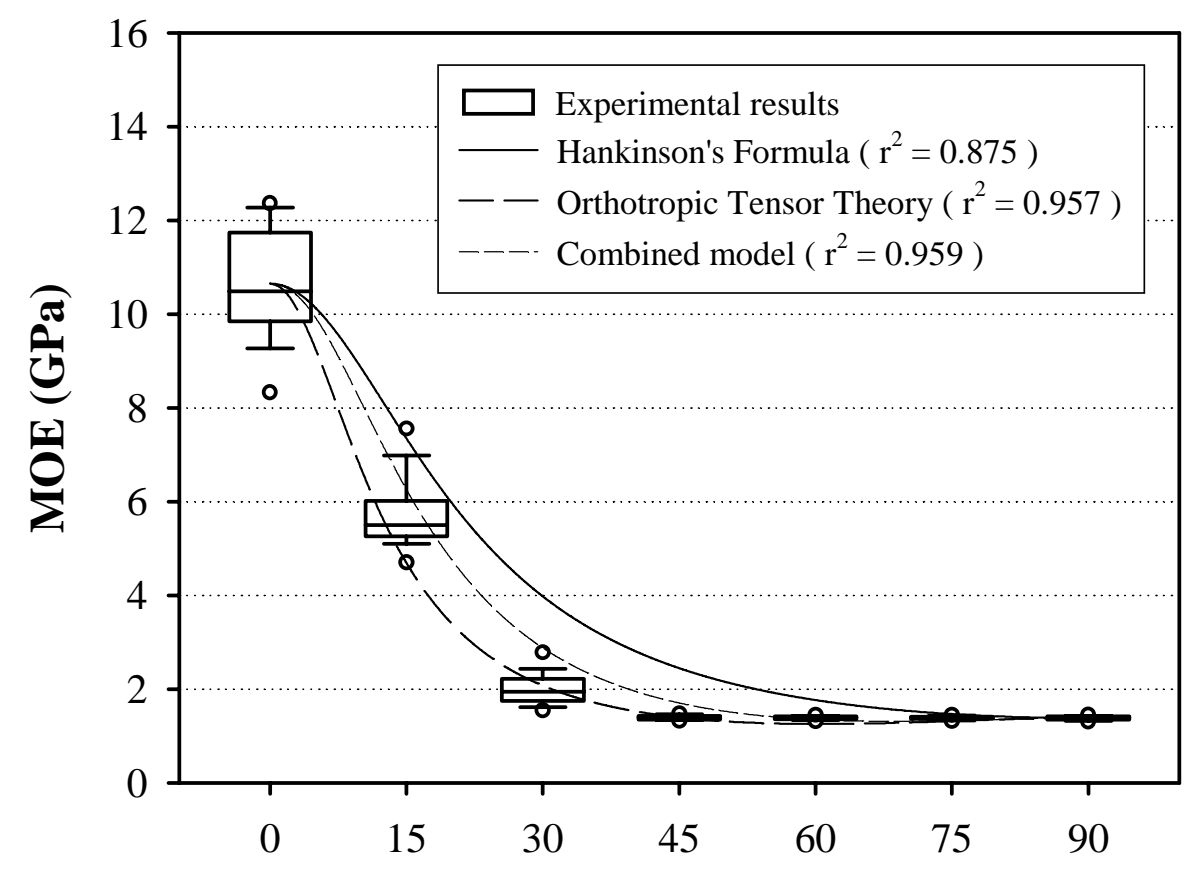

a.

Grain angle $\left(\varphi^{\circ}\right)$

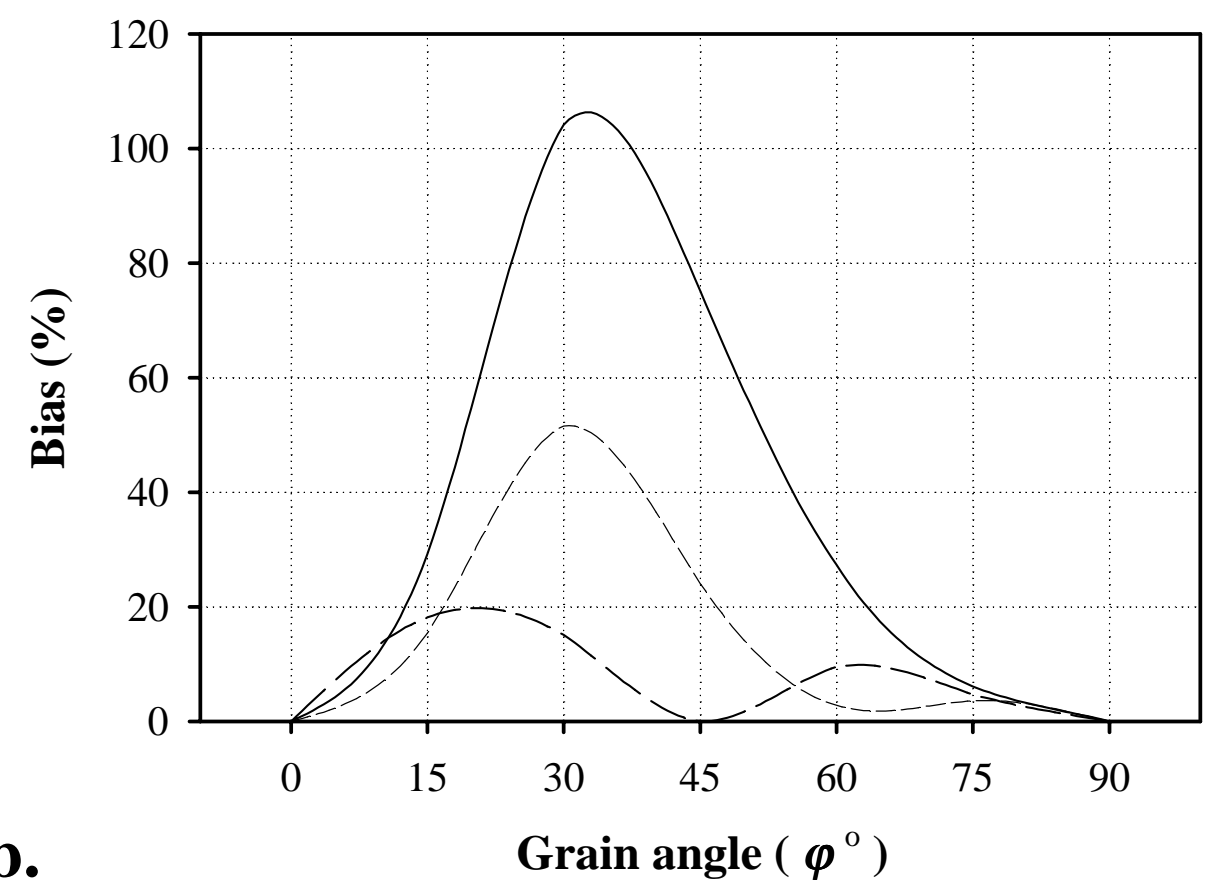

Figure 6.11 - Orthotropic dynamic MOE of red oak structural veneer sheets a. - experimental and model predicted values

b. - Average Absolute Percentage Bias associated with the different models 


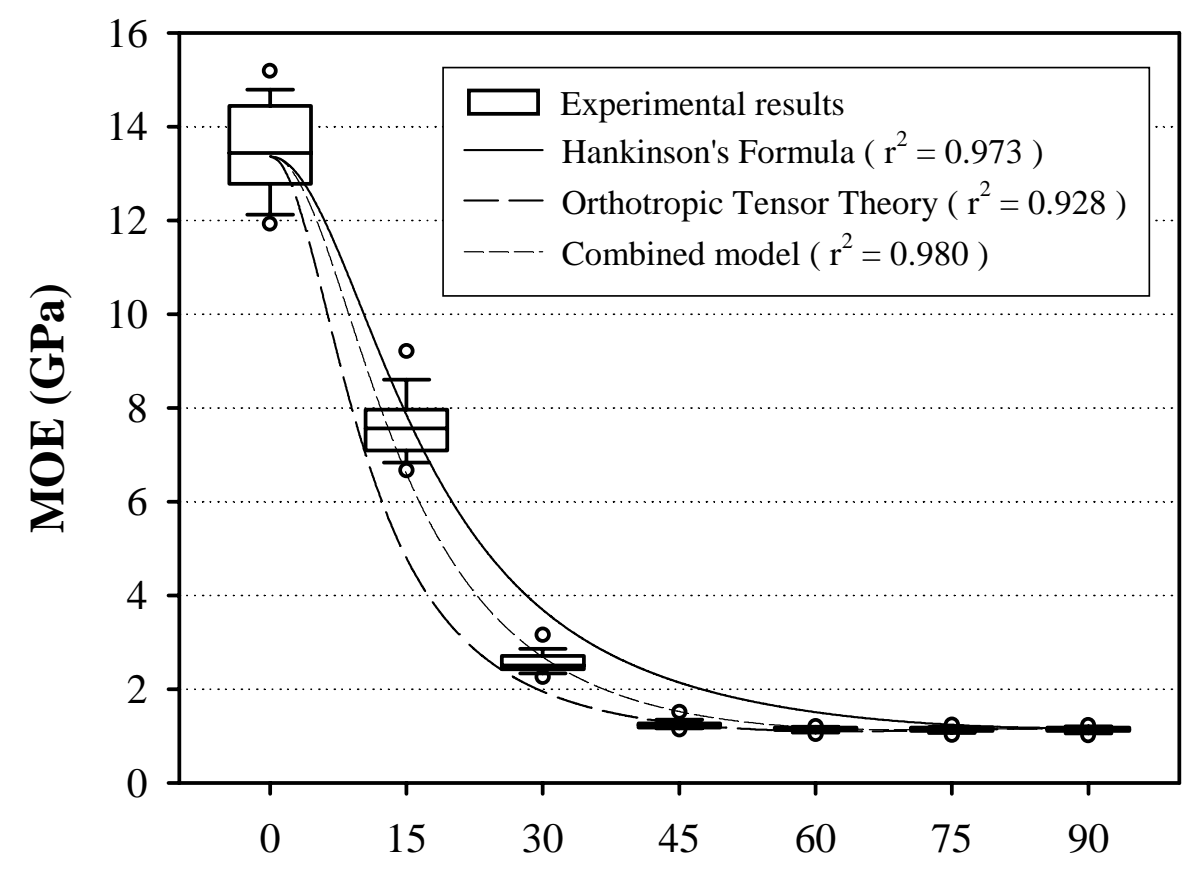

a.

Grain angle $\left(\varphi^{\circ}\right)$

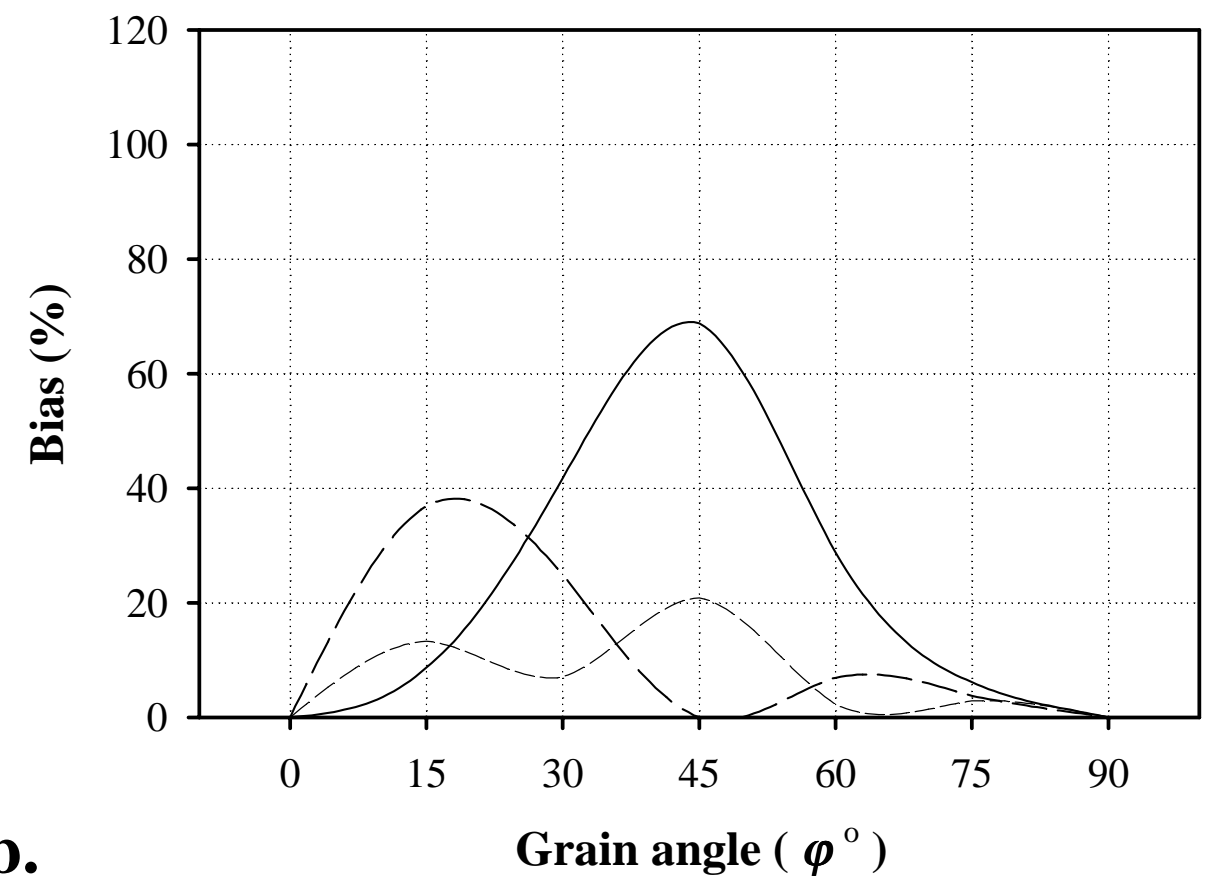

Figure 6.12 - Orthotropic dynamic MOE of yellow-poplar structural veneer sheets a. - experimental and model predicted values b. - Average Absolute Percentage Bias associated with the different models 
In general, measured dynamic MOE values decreased sharply with grain angle, under $45^{\circ}$ grain orientation. Above this angle, MOE leveled off, and there was little additional decrease. These observations agree well with the results of the orthotropic compression MOE determination (section 6.1.3.) One-way ANOVA revealed significant effect of grain angle for each species. Tukey's multiple comparison test, however, failed to detect significant differences between the MOE values above $45^{\circ}$ grain angle. (APPENDIX B provides a detailed summary of the ANOVA results.)

The two prediction models discussed in section 4.1 .3 were evaluated by applying them to each specimen individually. A measure of the model's efficiency is the average absolute percent bias (AAPB), that can be calculated the following way:

$$
\text { Bias }=\frac{\sum \frac{\left|\hat{E}_{\varphi i}-E_{\varphi i}\right|}{E_{\varphi i}}}{N} 100 \%
$$

where: $\hat{E}_{\varphi i}-$ MOE value predicted at grain angle $\varphi$ by applying Equations 4.13 or 4.14 on specimen $i$;

$E_{\varphi i} \quad-$ MOE measured on specimen $i$ at grain angle $\varphi$;

$N \quad-$ total number of specimens.

Table 6.8 contains the calculated AAPB by species, prediction model and grain angle. Figures $6.10 \mathrm{~b}$ through $6.12 \mathrm{~b}$ illustrate how bias changes throughout the grain angle range. 
Table 6.8. - Average absolute percent bias (AAPB) values associated with the three models

\begin{tabular}{|c|c|c|c|c|}
\hline & \multirow[b]{2}{*}{$\varphi^{\circ}$} & \multicolumn{3}{|c|}{ Average absolute bias (\%) } \\
\hline & & $\begin{array}{l}\text { Hankinson's } \\
\text { formula }\end{array}$ & $\begin{array}{l}\text { Orthotropic } \\
\text { tensor theory }\end{array}$ & $\begin{array}{c}\text { Combined } \\
\text { model }\end{array}$ \\
\hline \multirow{7}{*}{ ASPEN } & 0 & 0.0 & 0.0 & 0.0 \\
\hline & 15 & 7.1 & 31.3 & 12.9 \\
\hline & 30 & 24.3 & 24.0 & 6.4 \\
\hline & 45 & 49.4 & 0.0 & 14.5 \\
\hline & 60 & 27.3 & 4.3 & 6.1 \\
\hline & 75 & 6.0 & 1.8 & 1.5 \\
\hline & 90 & 0.0 & 0.0 & 0.0 \\
\hline \multirow{7}{*}{ OAK } & 0 & 0.0 & 0.0 & 0.0 \\
\hline & 15 & 29.2 & 18.2 & 15.4 \\
\hline & 30 & 104.1 & 15.0 & 51.6 \\
\hline & 45 & 75.1 & 0.0 & 24.1 \\
\hline & 60 & 27.3 & 9.5 & 2.8 \\
\hline & 75 & 6.1 & 4.6 & 3.6 \\
\hline & 90 & 0.0 & 0.0 & 0.0 \\
\hline \multirow{7}{*}{$\begin{array}{l}\text { YELLOW- } \\
\text { POPLAR }\end{array}$} & 0 & 0.0 & 0.0 & 0.0 \\
\hline & 15 & 8.7 & 36.8 & 13.3 \\
\hline & 30 & 41.7 & 25.0 & 7.0 \\
\hline & 45 & 68.8 & 0.0 & 20.9 \\
\hline & 60 & 28.8 & 6.9 & 2.3 \\
\hline & 75 & 6.2 & 3.7 & 2.9 \\
\hline & 90 & 0.0 & 0.0 & 0.0 \\
\hline
\end{tabular}

The orthotropic tensor theory exhibits little bias at higher grain angles, above $45^{\circ}$. At lower grain orientations, however, it tends to underestimate MOE by $20 \sim 40 \%$. Since MOE values are relatively high in this region, this translates into a large absolute bias. increases rapidly to a relatively high peak at $\varphi=30^{\circ}$ or $45^{\circ}$. The maximum bias is especially large in the case of oak. Since MOE decreases significantly at these grain orientations, this corresponds to a smaller maximum absolute bias than that of the orthotropic tensor theory. 
The above considerations suggest that a combination of the two models could improve the prediction throughout the whole grain angle range. The following is a possible combination that works well:

$$
\hat{E}_{C}=\hat{E}_{O} \frac{\sqrt{\varphi}}{\sqrt{\pi / 2}}+\hat{E}_{H} \frac{\sqrt{\pi / 2}-\sqrt{\varphi}}{\sqrt{\pi / 2}}
$$

where: $\hat{E}_{C}$ - predicted MOE at grain angle $\varphi$ - combined model;

$\hat{E}_{O} \quad$ - predicted MOE at grain angle $\varphi$ - Orthotropic Tensor Theory (Eq. 4.13).

$\hat{E}_{H} \quad-$ predicted MOE at grain angle $\varphi$ - Hankinson's Fromula (Eq. 4.14);

Figures 6.10 through 6.12 and Table 6.8 show the AAPB associated with the combined model, as well. Clearly, this model provides very good prediction throughout the whole range, for aspen and yellow-poplar. Although for oak, its bias is still rather large at $\varphi=30^{\circ}$, the general performance of the combination appears to be better than either of its components.

Figure 6.13 shows the relationship between the experimentally measured dynamic and static MOE. This graph includes the data points measured on both veneer and solid wood, for all three species. Theoretically, the ratio of $E_{D}$ and $E_{S}$ should depend only on the characteristic time of the determination (see Equation 4.17.) Since this does not change with species or orientation, the relationship should be linear, and the regression line should pass through the origin. The correlation parameters shown on Figure 6.13 a, however, show a different trend. The regression line does not pass through the origin, and the linearity of the data is questionable. The cause of this anomaly is most 

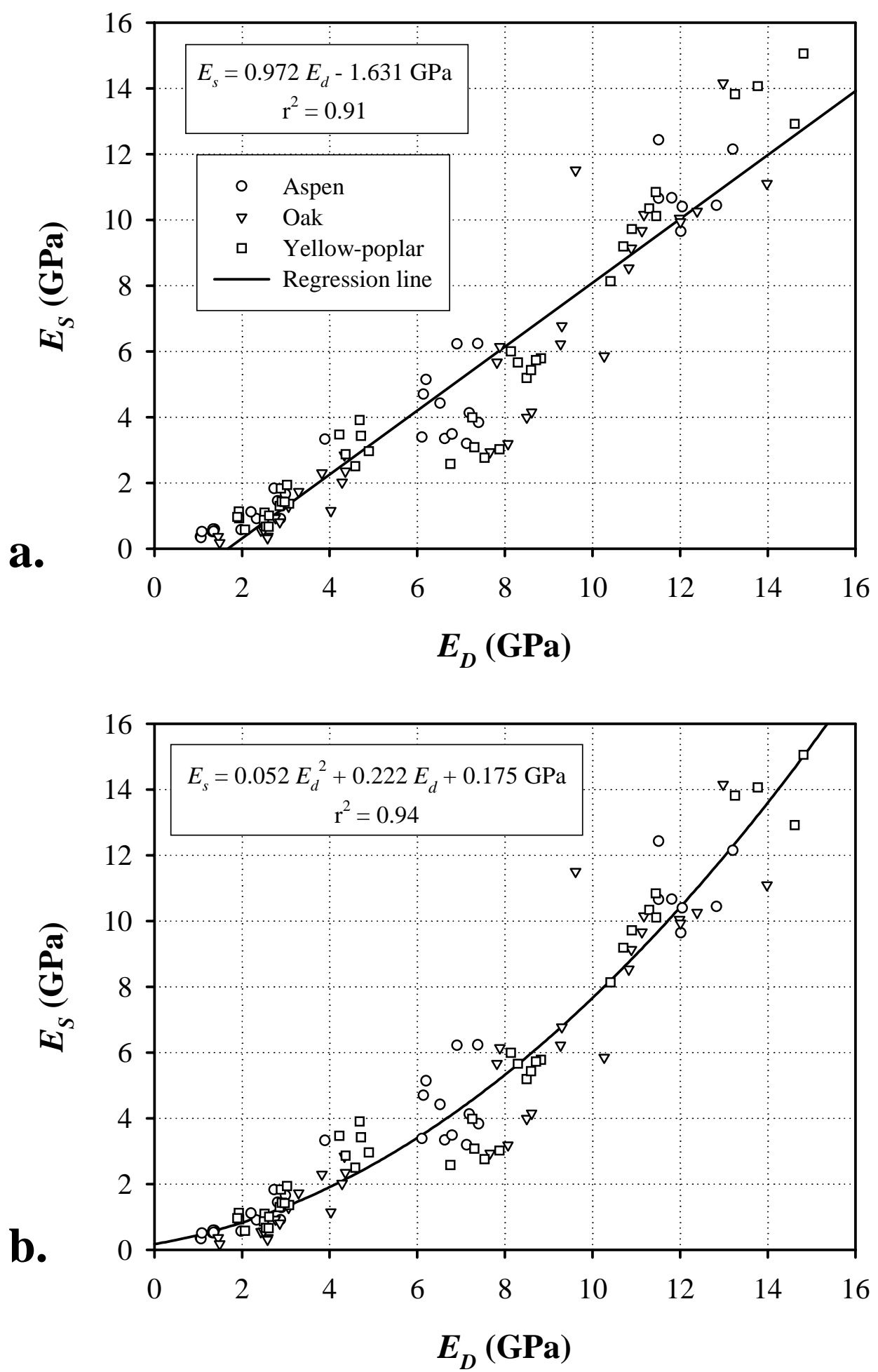

Figure 6.13 - Linear (a) and quadratic (b) relationship between the static and dynamic MOE of the three Appalachian species measured at various grain angles 
likely the high damping effect in veneer in non-longitudinal directions, which might have influenced the measured propagation time. A serious problem with the regression line depicted on Figure 6.13 a is that, according to this model, dynamic MOE values measured at higher grain deviations correspond to negative static MOE.

Figure 6.13 b shows the same graph with a second order regression line. This model describes the data significantly better than did the line depicted in Figure 6.13 a, as the improved $\mathrm{r}^{2}$ value indicates. It is also apparent that, using the quadratic model, positive static MOE values are calculated for any dynamic MOE. While there is no theoretical justification for using a second order regression model, it appears that the quadratic equation might be best for estimating the static MOE from its dynamic counterpart.

\subsection{The effect of densification on the MOE of veneer}

Analyzing the relationship between densification and MOE increase required the calculation of two parameters:

$$
\Delta E=\frac{E_{d}-E_{o}}{E_{o}} 100 \%
$$

where: $\Delta E \quad-$ MOE increase (\%);

$E_{o}, E_{d}-\mathrm{MOE}$ measured before and after densification, and

$$
\Delta \rho=\frac{\rho_{d}-\rho_{o}}{\rho_{o}} 100 \%,
$$

where: $\Delta \rho \quad-$ densification (\%);

$\rho_{o}, \rho_{d}$-density before and after densification. 


\subsubsection{The effect of densification on the dynamic MOE}

One densification value was calculated, while a separate $\Delta E$ value was obtained in every measurement direction, for each specimen. Individual analysis of the correlation between $\Delta \rho$ and $\Delta E$ at each grain orientation indicated that grain angle significantly affected the relationship between these parameters. Closer examination of the data revealed that differences occurred mainly at the $15^{\circ} \sim 45^{\circ}$ grain angle range, due to the presence of outliers in the experimental data. These were caused by the increased uncertainty of the measurements at these grain angles. After the elimination of outlying values, the relationship between $\Delta E$ and $\Delta \rho$ was similar at every grain angle level. For each species, $\Delta E$ values measured at different grain angles were pooled together for further analysis.

As expected, $\Delta E$ tends to increase with $\Delta \rho$, for all of the species, (Figure 6.14). Two considerations were used to describe the relationship between these parameters. First, the regression line must pass through the origin, because the MOE of non-densified veneer remains unchanged. The second assumption is based on the consideration, that, although squeezing more material into the same volume increases the resistance to deformation, cell walls suffer some damages in the process. The cumulative effect of the damages decreases the rate of improvement progressively. A second order polynomial regression, with zero $y$-intercept and a negative second order coefficient, is adequate to meet the above criteria. 

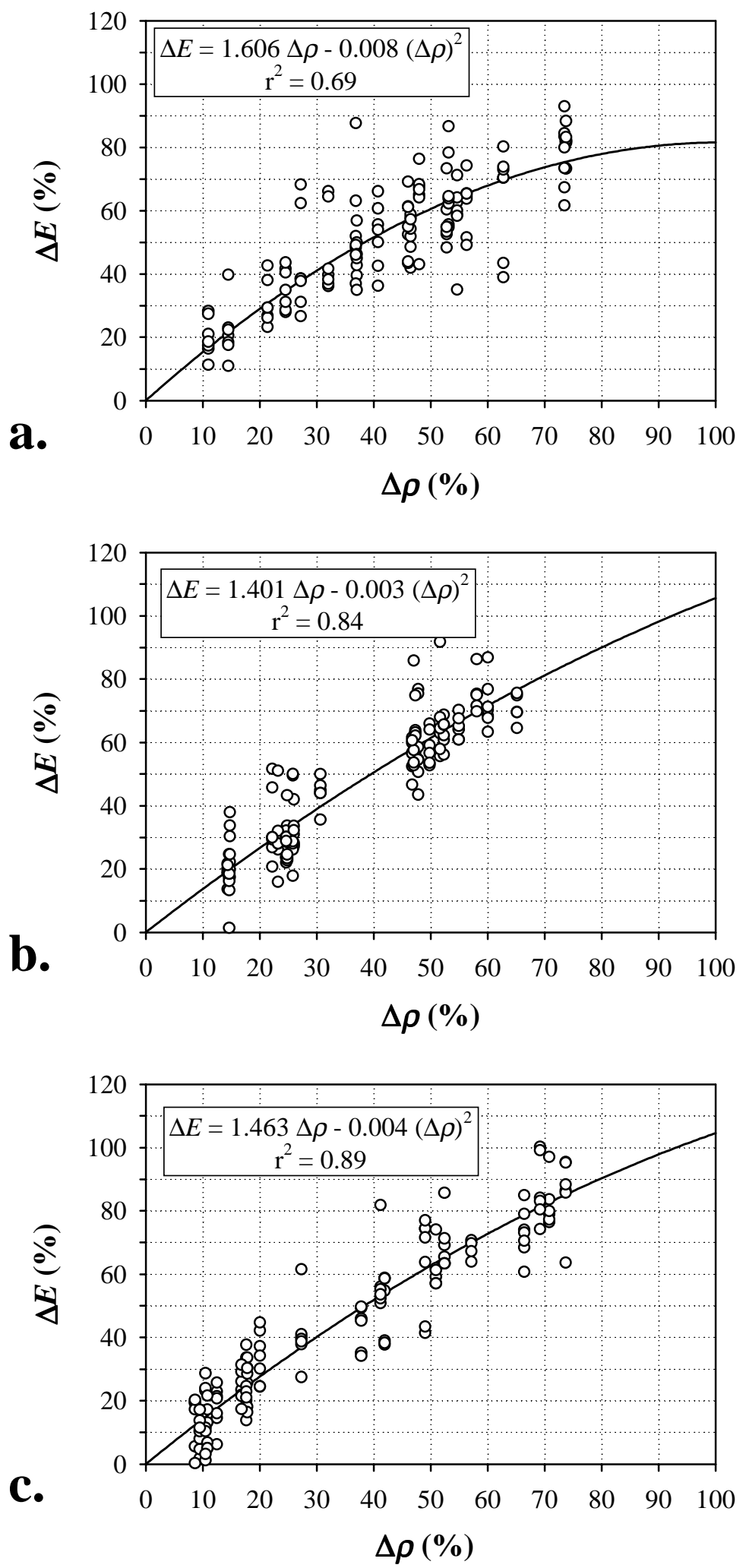

Figure 6.14 - Relationship between dynamic MOE increase and densification for aspen $(\mathbf{a})$, red oak (b) and yellow-poplar (c) 
Table 6.9 - Parameters of the first and second order regression equations, and the associated $r^{2}$ values

\begin{tabular}{|c|c|c|c|c|c|}
\hline & $\begin{array}{c}\text { Regression } \\
\text { type }\end{array}$ & $b_{0}$ & $b_{1}$ & $b_{2}$ & $\mathbf{r}^{2}$ \\
\hline \multicolumn{6}{|c|}{ 1.) BASED ON DYNAMIC MOE } \\
\hline ASPEN & $\begin{array}{l}\text { Linear }^{1} \\
\text { Quadratic }^{2}\end{array}$ & $\begin{array}{l}0 \\
0\end{array}$ & $\begin{array}{l}1.170 \\
1.606\end{array}$ & $\begin{array}{r}--- \\
-0.008\end{array}$ & $\begin{array}{l}0.61 \\
0.69\end{array}$ \\
\hline RED OAK & $\begin{array}{l}\text { Linear } \\
\text { Quadratic }\end{array}$ & $\begin{array}{l}0 \\
0\end{array}$ & $\begin{array}{l}1.232 \\
1.401\end{array}$ & $\begin{array}{r}--- \\
-0.003\end{array}$ & $\begin{array}{l}0.83 \\
0.84\end{array}$ \\
\hline $\begin{array}{l}\text { YELLOW- } \\
\text { POPLAR }\end{array}$ & $\begin{array}{l}\text { Linear } \\
\text { Quadratic }\end{array}$ & $\begin{array}{l}0 \\
0\end{array}$ & $\begin{array}{l}1.224 \\
1.463\end{array}$ & $\begin{array}{r}--- \\
-0.004\end{array}$ & $\begin{array}{l}0.88 \\
0.89\end{array}$ \\
\hline \multicolumn{6}{|c|}{ 2.) BASED ON STATIC MOE } \\
\hline $\begin{array}{l}\text { YELLOW- } \\
\text { POPLAR }\end{array}$ & $\begin{array}{l}\text { Linear } \\
\text { Quadratic }\end{array}$ & $\begin{array}{l}0 \\
0\end{array}$ & $\begin{array}{l}0.805 \\
1.332\end{array}$ & $\begin{array}{r}--- \\
-0.005\end{array}$ & $\begin{array}{l}0.36 \\
0.45\end{array}$ \\
\hline
\end{tabular}

${ }^{\mathrm{I}}$ Regression model: $\Delta E=b_{0}+b_{1} \Delta \rho$

${ }^{2}$ Regression model: $\Delta E=b_{0}+b_{1} \Delta \rho+\mathrm{b}_{2} \Delta \rho^{2}$

Figure 6.14 contains plots of the fitted quadratic regression line, for all of the species. It is apparent from the plots, that $\Delta E$ does indeed increase at a decreasing rate. This tendency is especially pronounced in aspen. Table 6.9 shows the parameters and $\mathrm{r}^{2}$ values of the second order polynomial regression, for the three species. This table contains the slope and $r^{2}$ values of a linear regression equation, too $\left(y_{0}=0\right)$. The coefficient of determination values indicate that the quadratic model performs better for aspen. Even though the second order model does not present significant improvement for the other two species, it probably approaches physical reality better. 


\subsubsection{Validation by static tensile MOE measurements}

$\Delta E$ was calculated for each densified specimen, using its measured static MOE value, and the MOE of the undensified control, as $E_{d}$ and $E_{o}$, respectively, in Equation 6.4. Figure 6.15 shows the increase of the static MOE of yellow poplar as a function of the measured densification, in the longitudinal direction. The data points exhibit a similar trend as observed in the previous section; MOE increases at a decreasing rate. This justifies the use of the second order regression model described in section 6.2.1, which is included on Figure 6.15. Table 6.9 shows the parameters of the curve, along with that of the linear regression.

It is apparent from the results that the stress-wave timer indicated a higher MOE increase than did static testing, at any densification level (Figure 6.16.) The most likely explanation for this phenomenon is that a slight modification, applied to the stress-wave setup before testing the densified specimens, improved the coupling of the accelerometers to the material. This, in turn, decreased the measured propagation times, and introduced a systematic error in the dynamic MOE measurement.

Unfortunately, static testing results apply to yellow-poplar veneer only, in the longitudinal direction. A comparison of the measured static and dynamic MOE increase of yellow-poplar, as a function of densification, provided a modification factor that can be used to adjust the densification curves presented in section 6.2.1. One way to calculate such a factor is derived in APPENDIX $\boldsymbol{D}$. The calculation resulted in a modification factor of 1.24. Dividing the coefficients $b_{1}$ and $b_{2}$ by this number (that is, in effect, decreasing the prediction at any densification level by the same factor) 


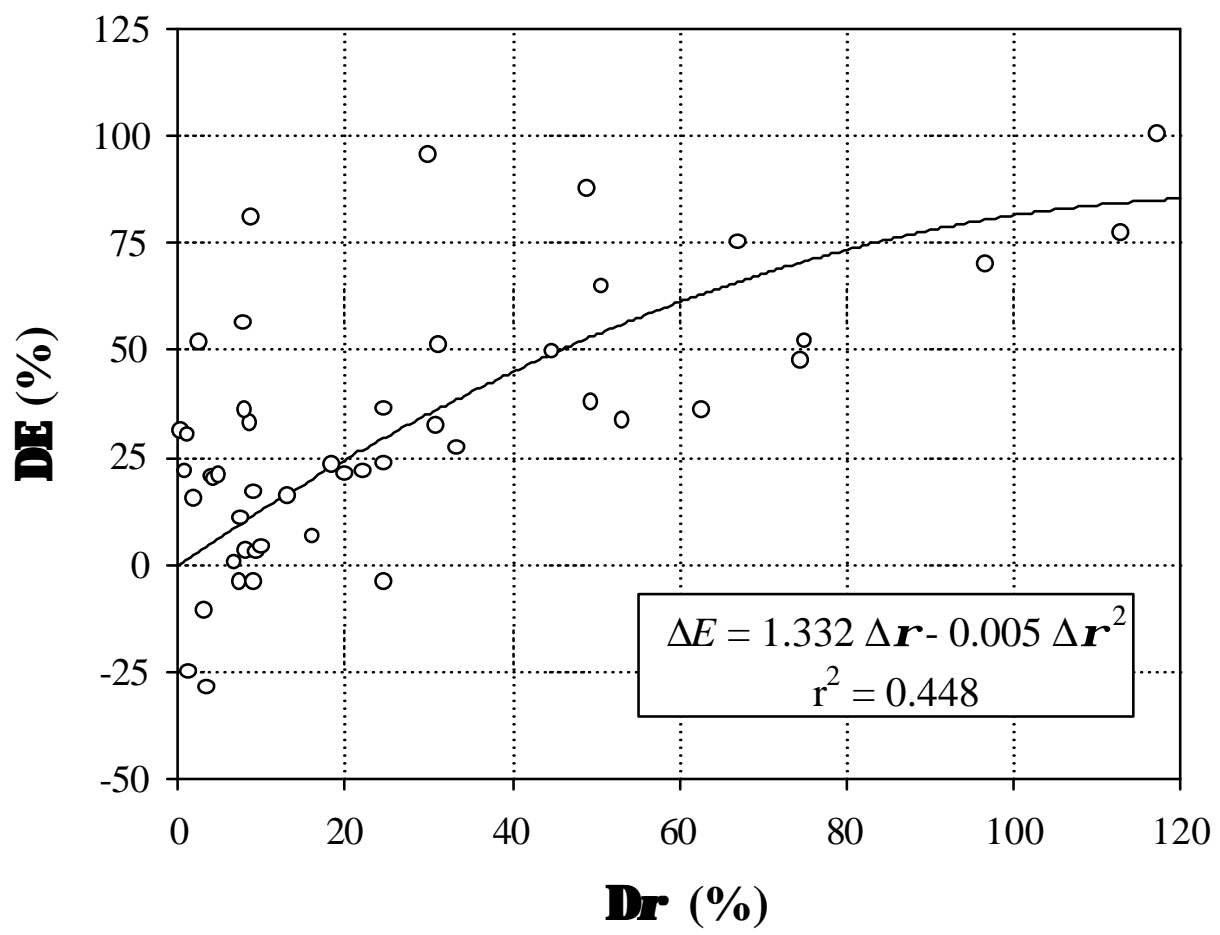

Figure 6.15 - Relationship between static MOE increase and densification for yellow-poplar veneer strands with longitudinal grain orientation

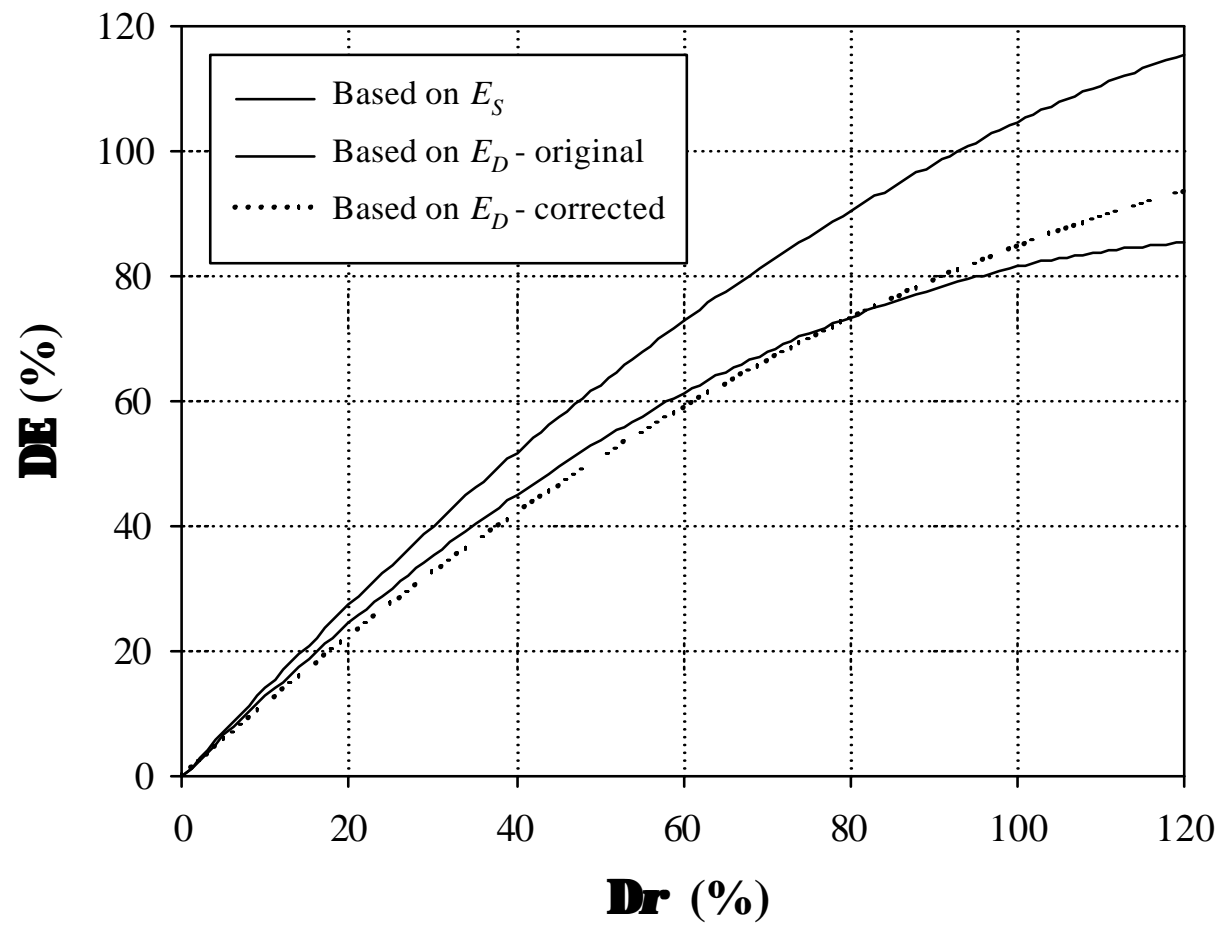

Figure 6.16 - Comparison of the static and dynamic densification curve of yellow-poplar. Modified dynamic densification curve 
results in more realistic $\Delta \rho \sim \Delta \mathrm{E}$ curve for yellow poplar, as shown on Figure 6.16. Because static densification curves are not available for aspen and red oak, their parameters can be corrected using the same factor.

\subsection{Mechanical properties of the composites}

\subsubsection{Physical properties of the composite lumber}

Table 6.10 provides the statistical summary of the measured physical properties of LVL and PSL. This table contains the moisture content, specific gravity and weight density of the composites at the standard environmental conditions $\left(21^{\circ} \mathrm{C}, 65 \% \mathrm{RH}\right)$, in which the small clear (shear and compression) specimens were kept. It also shows the moisture content of the bending specimens, which were conditioned using a different environment.

Table 6.10 - Summary statistics of the physical properties of LVL and PSL

\begin{tabular}{lcccccc}
\hline \hline & N & \multicolumn{2}{c}{ LVL } & \multicolumn{2}{c}{ PSL } \\
& Mean & $\mathbf{s}$ & Mean & s \\
\hline $21^{\circ} \mathrm{C}, 65 \%$ RH & & & & & \\
Moisture content & 30 & 12.06 & 0.22 & 11.36 & 0.11 \\
Specific gravity & 30 & 0.50 & 0.01 & 0.60 & 0.01 \\
Density ( kg/m $\left.{ }^{3}\right)$ & 30 & 566 & 11 & 673 & 16 \\
$25^{\circ} \mathrm{C}, 45 \%$ RH & & & & & \\
Moisture Content & 10 & 10.65 & 0.29 & 9.79 & 0.26 \\
\hline \hline
\end{tabular}




\subsubsection{Bending MOE of LVL and PSL}

Table 6.11 provides the statistical summary of the measured flatwise and edgewise bending MOE of LVL and PSL. Pairwise t-tests failed to show statistically significant differences between the flatwise and edgewise MOE, for either of the composite types. Another t-test indicated a statistically significant difference between the MOE of LVL and PSL. Analysis of variance on LVL's bending MOE provided the within- and between panel variance for LVL, which was $0.634 \mathrm{GPa}^{2}$ and $0.456 \mathrm{GPa}^{2}$, respectively (for the calculations see APPENDIX B.)

As Table 6.11 shows, LVL has a higher bending MOE than does PSL, in spite of the higher average densification of the latter. This may be due to several factors, such as the imperfect orientation of the strands, strand damage during hot pressing, or weaker gluelines in PSL, caused by its less regular structure.

Figures 6.17 $\mathbf{a}$ and $\mathbf{b}$ show the relationship between the edgewise and flatwise bending MOE of LVL and PSL, respectively. As the low $r^{2}$ values indicate, the correlation between the two parameters is relatively weak. This fact can be explained by the laminate theory. As the orientation of a beam changes, the distance of some

Table 6.11 - Flatwise and edgewise bending MOE of LVL and PSL (N=20)

\begin{tabular}{lrcrc}
\hline & \multicolumn{2}{c}{ LVL } & \multicolumn{2}{c}{ PSL } \\
\cline { 2 - 4 } \cline { 5 - 5 } & $\begin{array}{c}\boldsymbol{E}_{\text {edge }} \\
(\mathrm{GPa})\end{array}$ & $\begin{array}{c}\boldsymbol{E}_{\text {flat }} \\
(\mathrm{GPa})\end{array}$ & $\begin{array}{c}\boldsymbol{E}_{\text {edge }} \\
(\mathrm{GPa})\end{array}$ & $\begin{array}{c}\boldsymbol{E}_{\text {flat }} \\
(\mathrm{GPa})\end{array}$ \\
\hline Mean & 13.22 & 13.36 & 12.82 & 12.57 \\
$\boldsymbol{S}$ & 1.21 & 0.72 & 0.75 & 0.57 \\
Min & 11.85 & 12.20 & 11.18 & 11.64 \\
Max & 15.90 & 15.16 & 13.81 & 13.92 \\
\hline \hline
\end{tabular}



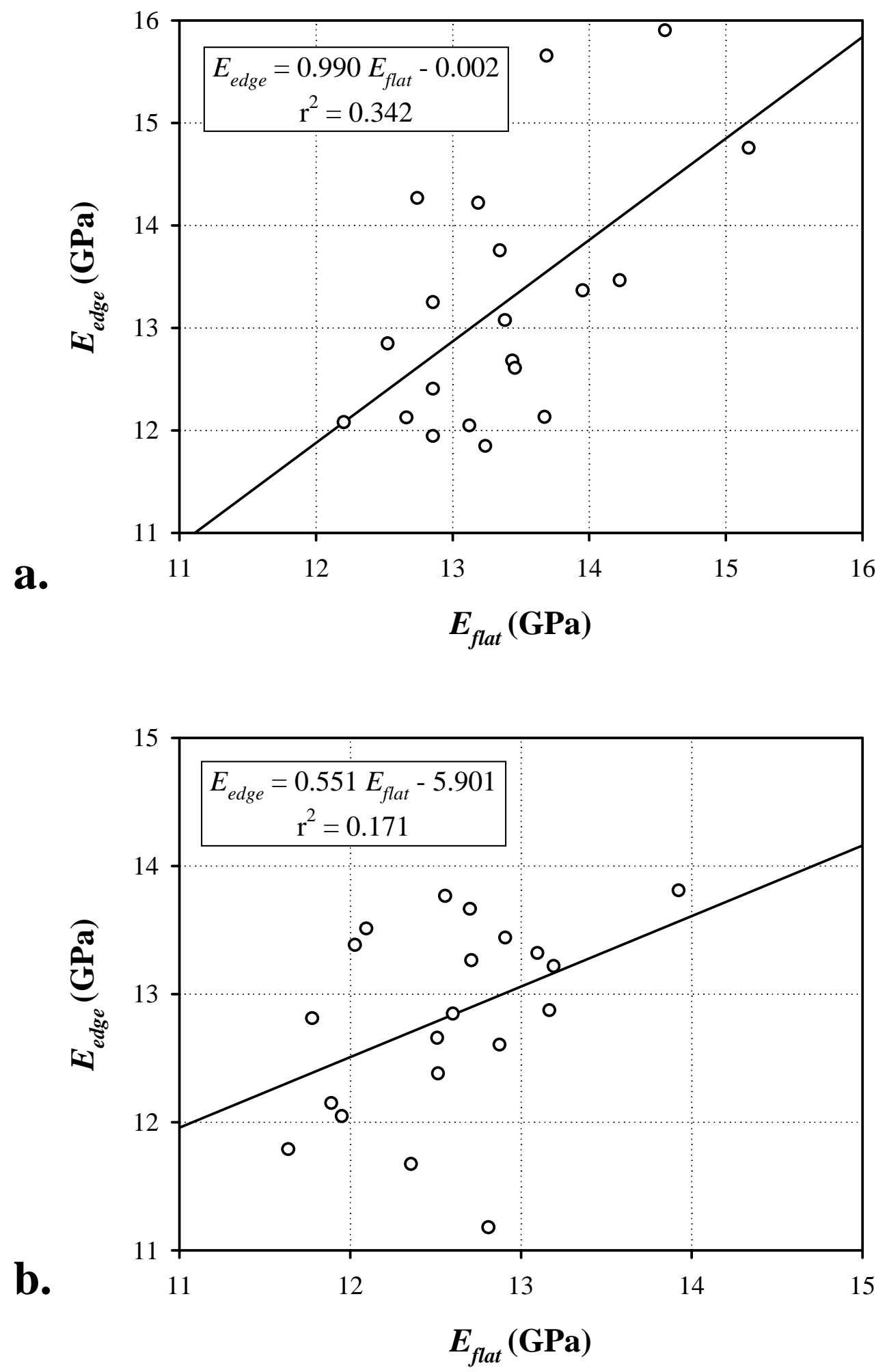

Figure 6.17 - Relationship between the edgewise and flatwise bending MOE of LVL (a) and PSL (b) 
constituents from the neutral plane changes, too, therefore their importance in determining the beam's overall bending MOE increases or diminishes. In some beams, stronger components are more deterministic in edgewise application, while weaker strands gain importance in flatwise orientation, or vice versa. It is probable that the slope of the regression line in PSL, which differs from unity considerably, is a result of this weak association, rather than a reflection of physical reality.

\subsubsection{Orthotropy of shear strength}

Table 6.12 contains the summary statistics of the measured shear strength of LVL and PSL at every combination of load and strand/layer orientation that the study incorporated. The examined composites exhibited similar tendencies of out-of-shearedplane failure, as did solid wood. Shear strength results reported in Table 6.12 should, therefore, be treated as apparent values.

Because the test did not include every angle combination, general tendencies are hard to observe in the data. Shear strength values typically decreased with increasing load orientation $\left(\varphi^{\prime}\right)$. When load was applied in the longitudinal $(x)$ direction, peak shear strength was experienced at $75^{\circ}$ strand or layer orientation $\left(\theta^{\prime}\right)$, in both LVL and PSL. This peak is especially pronounced in LVL. A similar tendency is observable in solid yellow-poplar timber (see Table 6.2 and Figure 6.3 a.)

Statistical data analysis included two-way ANOVA for the two composite types. Results indicated significant load and layer/strand orientation effect in both materials. The incomplete statistical design did not allow the assessment of the interaction effect. 
Table 6.12 - Summary statistics of the experimentally determined orthotropic shear strength of LVL and PSL

\begin{tabular}{|c|c|c|c|c|c|c|c|c|c|c|c|}
\hline \multirow{2}{*}{$\varphi^{\prime \circ}$} & \multirow{2}{*}{$\theta^{\prime} \circ$} & \multicolumn{5}{|c|}{ LVL } & \multicolumn{5}{|c|}{ PSL } \\
\hline & & $\begin{array}{c}\bar{\tau} \\
(\mathrm{MPa})\end{array}$ & $\begin{array}{c}\mathbf{s} \\
(\mathrm{MPa})\end{array}$ & $\begin{array}{c}\min \\
(\mathrm{MPa})\end{array}$ & $\begin{array}{c}\max \\
(\mathrm{MPa})\end{array}$ & $\mathbf{N}^{\mathrm{a}}$ & $\begin{array}{c}\bar{\tau} \\
(\mathrm{MPa})\end{array}$ & $\begin{array}{c}\mathbf{s} \\
(\mathrm{MPa})\end{array}$ & $\underset{(\mathrm{MPa})}{\min }$ & $\begin{array}{c}\max \\
(\mathrm{MPa})\end{array}$ & $\mathbf{N}$ \\
\hline 0 & 0 & 6.77 & 0.62 & 5.67 & 7.47 & 9 & 6.59 & 0.84 & 5.77 & 8.14 & 9 \\
\hline 0 & 15 & 6.55 & 0.99 & 4.64 & 7.92 & 9 & 6.33 & 0.78 & 5.52 & 7.64 & 9 \\
\hline 0 & 30 & 5.95 & 0.79 & 5.16 & 7.11 & 9 & 5.99 & 0.60 & 5.10 & 6.94 & 9 \\
\hline 0 & 45 & 6.17 & 1.29 & 4.32 & 8.30 & 9 & 7.38 & 1.00 & 6.04 & 8.80 & 9 \\
\hline 0 & 60 & 6.55 & 1.26 & 4.75 & 8.68 & 9 & 7.45 & 1.13 & 5.28 & 8.77 & 9 \\
\hline 0 & 75 & 11.04 & 1.10 & 9.27 & 12.42 & 9 & 8.33 & 0.58 & 7.40 & 9.38 & 9 \\
\hline 0 & 90 & 7.09 & 1.27 & 5.39 & 8.62 & 9 & 7.79 & 1.25 & 6.23 & 9.76 & 9 \\
\hline 15 & 0 & 6.77 & 1.02 & 5.33 & 8.45 & 9 & 6.12 & 0.81 & 5.30 & 7.58 & 9 \\
\hline 30 & 0 & 4.95 & 1.02 & 2.78 & 5.95 & 9 & 4.96 & 1.60 & 1.05 & 6.12 & 9 \\
\hline 45 & 0 & 4.30 & 0.71 & 3.08 & 5.57 & 9 & 4.22 & 0.34 & 3.64 & 4.75 & 9 \\
\hline 60 & 0 & 3.33 & 0.50 & 2.61 & 4.19 & 9 & 3.26 & 0.86 & 1.60 & 4.66 & 9 \\
\hline 75 & 0 & 2.49 & 0.35 & 2.08 & 3.09 & 9 & 2.69 & 0.64 & 1.96 & 3.88 & 9 \\
\hline 90 & 0 & 2.80 & 0.40 & 2.40 & 3.48 & 9 & 2.15 & 0.26 & 1.85 & 2.74 & 9 \\
\hline 15 & 90 & 6.52 & 0.48 & 5.84 & 7.32 & 9 & 7.72 & 1.68 & 4.37 & 9.57 & 9 \\
\hline 30 & 90 & 5.52 & 0.59 & 4.21 & 6.29 & 9 & 8.28 & 2.27 & 3.83 & 12.10 & 9 \\
\hline 45 & 90 & 4.97 & 0.63 & 3.90 & 5.69 & 9 & 4.64 & 0.47 & 3.92 & 5.25 & 9 \\
\hline 60 & 90 & 3.97 & 0.65 & 3.04 & 5.41 & 9 & 4.21 & 0.43 & 3.61 & 4.76 & 9 \\
\hline 75 & 90 & 2.91 & 0.41 & 2.44 & 3.77 & 9 & 3.35 & 0.33 & 2.92 & 3.82 & 9 \\
\hline 90 & 90 & 3.04 & 0.29 & 2.73 & 3.70 & 9 & 2.94 & 0.47 & 2.16 & 3.66 & 9 \\
\hline 90 & 45 & 2.58 & 0.40 & 2.04 & 3.07 & 8 & 3.09 & 0.43 & 2.05 & 3.55 & 8 \\
\hline
\end{tabular}

a - sample size 
The effectiveness of the three prediction models presented in section 4.1.1, was evaluated, using $r^{2}$ analysis (Equation 6.1.) Table 6.13 shows the $r^{2}$ values associated with the three models. This table also indicates the $n$ values used in the Modified Hankinson formula (Equation 4.7), which were established by curve fitting, using the whole experimental database. Figure 6.18 shows the prediction of the orthotropic tensor theory and the modified Hankinson formula for both composite types in a threedimensional Cartesian coordinate system, along with the experimental mean values, to facilitate visual appraisal.

The results show that the orthotropic tensor theory and the modified Hankinson formula are suitable for describing the orthotropy of wood based composite lumber. The latter fits experimental values, because it can predict high shear strength values at $\varphi=15^{\circ}$, which occur in LVL and PSL, as well as solid wood. Both model provided relatively low $\mathrm{r}^{2}$ values, when predicting the shear strength of LVL. Figure $\mathbf{6 . 1 8}$ reveals the reason for this; the models were unable to estimate the high shear strength values experienced in the longitudinal direction, at $\theta^{\prime}=75^{\circ}$. Apart from this point, both models provide excellent fit to the experimental shear strength of LVL.

Table 6.13 - Coefficients of determination provided by the various prediction models

\begin{tabular}{ccccc}
\hline \hline Species & $\begin{array}{c}\text { Orthotropic } \\
\text { tensor theory } \\
\mathbf{r}^{\mathbf{2}}\end{array}$ & $\begin{array}{c}\text { Quadratic } \\
\text { formula } \\
\mathbf{r}^{\mathbf{2}}\end{array}$ & $\begin{array}{c}\text { Modified Hankinson } \\
\text { formula }\end{array}$ \\
\hline LVL & 0.68 & 0.66 & $\mathbf{n}$ & $\mathbf{r}^{\mathbf{2}}$ \\
PSL & 0.59 & 0.55 & 2.17 & 0.69 \\
\hline
\end{tabular}

n $\rightarrow$ the power in the Modified Hankinson formula 


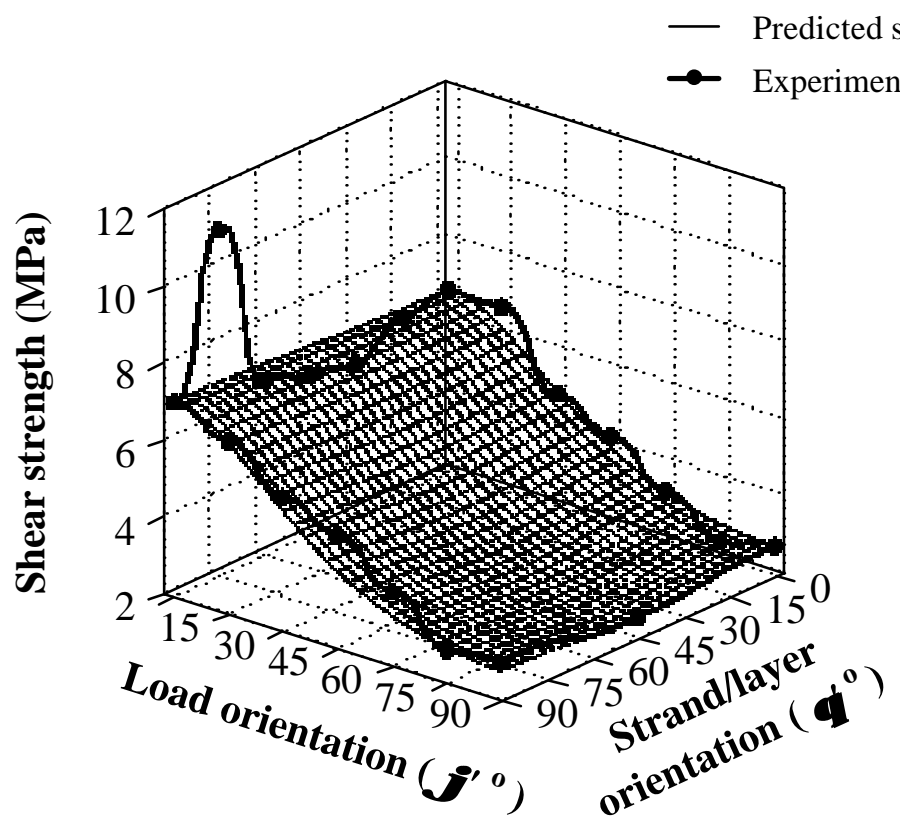

a. - LVL - Orthotropic tensor theory

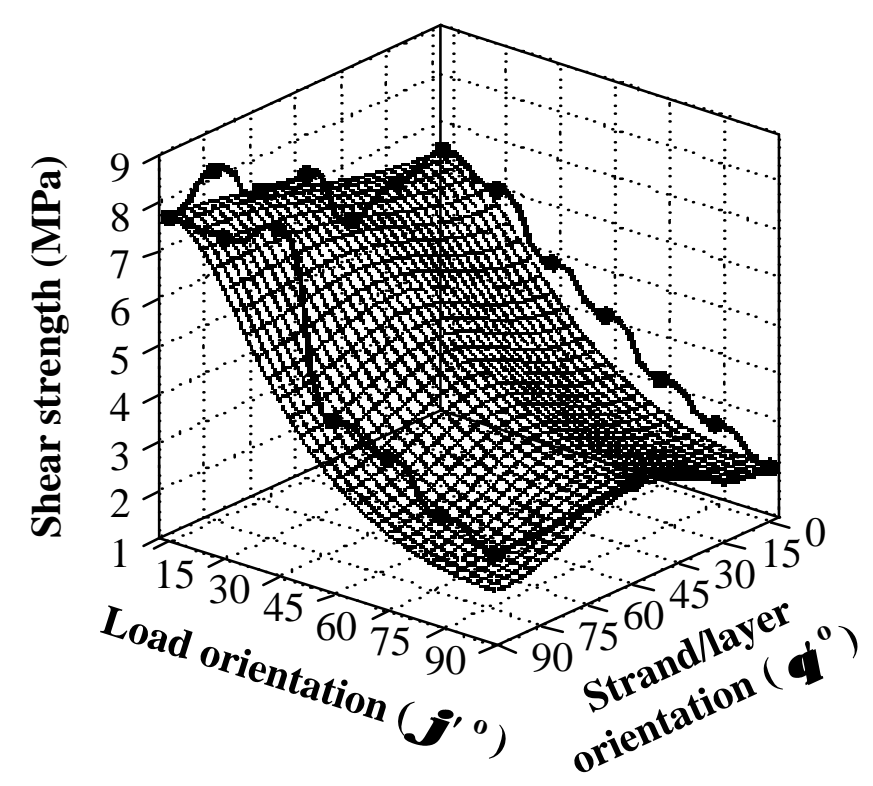

a. - PSL - Orthotropic tensor theory

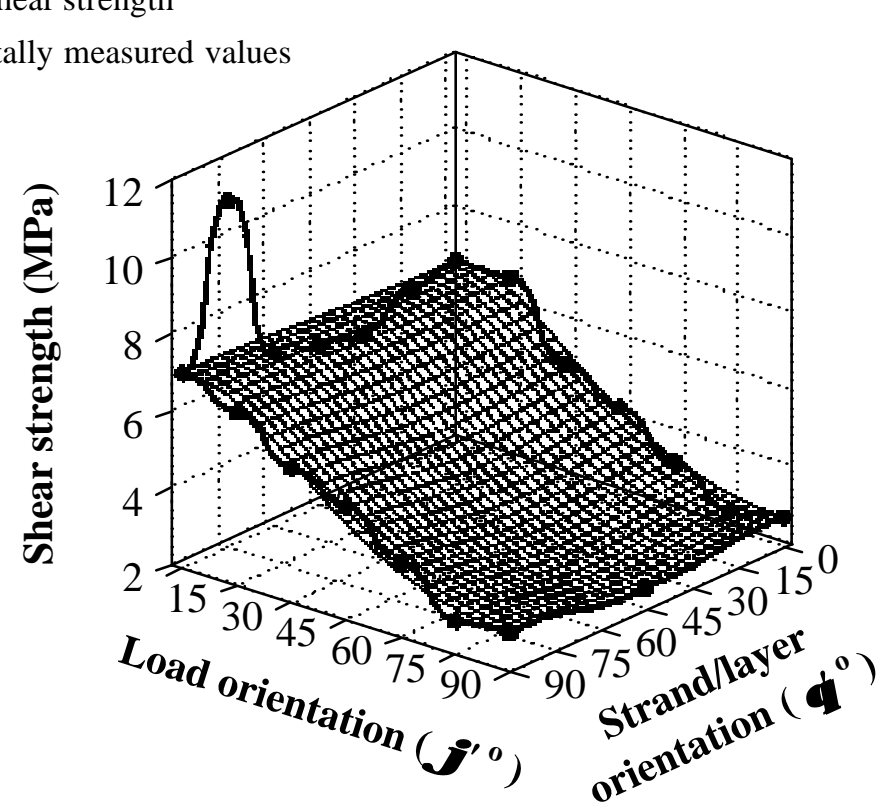

b. - LVL - Modified Hankinson formula

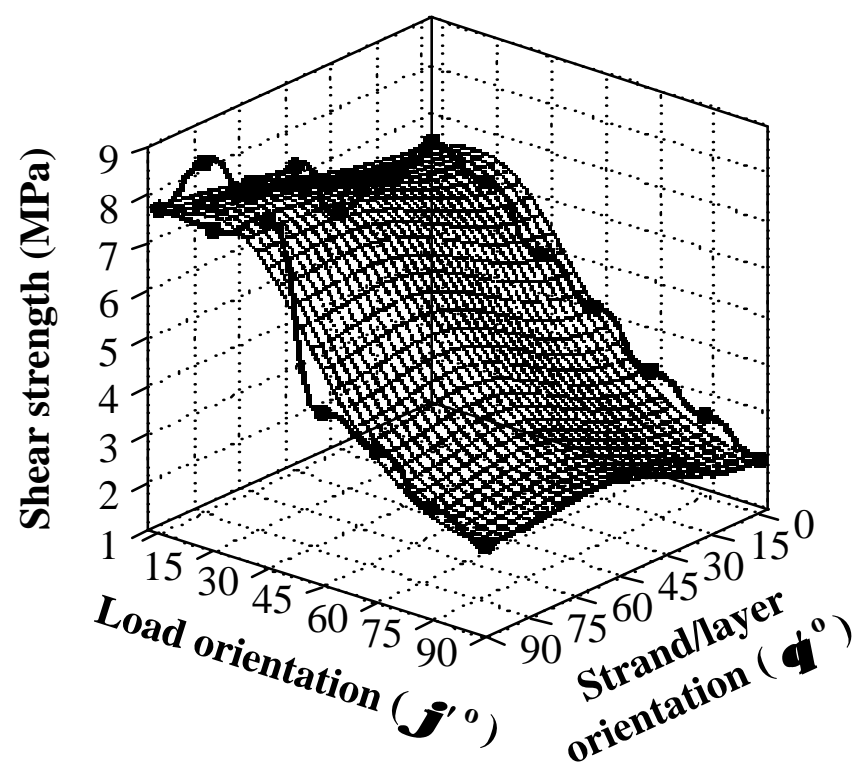

b. - PSL - Modified Hankinson formula

Figure 6.18 - Orthotropic diagrams of the shear strength of LVL and PSL 
The predictions of the Quadratic formula were, again, inferior to those of the other two models. It appears that, while this formula might be best to describe the theoretical shear strength of wood and composite lumber, the other theories approach apparent shear strength values, measured by traditional methods, better.

\subsubsection{Orthotropy of compression elasticity}

In the duration of the compression tests (up to 0.1 in displacement), very few specimens failed or reached the stress plateau indicated in Figure 6.4 b. For this reason, compression strength is unavailable for the composites. Specimens with sloping grain that had been re-glued to provide the necessary specimen length, did not show apparent signs of shear dislocation along the glueline. Specimens that did reach failure were sheared inside the composites, rather than along this interface. This lead to the conclusion that displacement data was not significantly influenced by the practice of re-gluing, and that collected data should produce valid MOE values.

Table 6.14 provides the summary statistics of the compression MOE of LVL and PSL in the six measured directions. Similarly to the elastic properties of solid wood, composite MOE dropped considerably between $\varphi^{\prime}=0^{\circ}$ and $\varphi^{\prime}=45^{\circ}$, but showed little change as load orientation increased further. Moreover, measured data indicates some negative correlation between layer/strand orientation $\left(\theta^{\prime}\right)$ and compression $\mathrm{MOE}$ at $\varphi^{\prime}=$ $45^{\circ}$ and $\varphi^{\prime}=90^{\circ}$. The low number of measurement points, however, does not warrant broad conclusions based on this observation. 
Table 6.14 - Summary statistics of the compression MOE of the two composites

\begin{tabular}{llrrrrrr}
\hline \hline & \multicolumn{1}{c}{$\begin{array}{c}E_{x} \\
(\mathrm{GPa})\end{array}$} & \multicolumn{1}{c}{$\begin{array}{c}E_{y} \\
(\mathrm{GPa})\end{array}$} & \multicolumn{1}{c}{$\begin{array}{c}E_{z} \\
(\mathrm{GPa})\end{array}$} & \multicolumn{1}{c}{$\begin{array}{c}E_{45^{\circ}}^{0^{\circ}} \\
(\mathrm{GPa})\end{array}$} & \multicolumn{1}{c}{$\begin{array}{c}E_{45^{\circ}}^{90^{\circ}} \\
(\mathrm{GPa})\end{array}$} & \multicolumn{1}{c}{$\begin{array}{c}E_{90^{\circ}}^{40^{\circ}} \\
(\mathrm{GPa})\end{array}$} \\
\hline \multirow{4}{*}{$\mathbf{L V L}$} & Mean & $\mathbf{1 1 . 8 7}$ & $\mathbf{0 . 4 4}$ & $\mathbf{0 . 3 7}$ & $\mathbf{0 . 8 7}$ & $\mathbf{0 . 8 4}$ & $\mathbf{0 . 2 9}$ \\
& $\mathrm{s}$ & 1.06 & 0.03 & 0.03 & 0.07 & 0.06 & 0.05 \\
& Min & 9.71 & 0.41 & 0.31 & 0.76 & 0.75 & 0.23 \\
& Max & 13.34 & 0.49 & 0.42 & 1.01 & 0.96 & 0.35 \\
& Mean & $\mathbf{1 3 . 2 0}$ & $\mathbf{0 . 4 8}$ & $\mathbf{0 . 2 3}$ & $\mathbf{1 . 0 8}$ & $\mathbf{0 . 6 4}$ & $\mathbf{0 . 3 0}$ \\
& $\mathrm{s}$ & 2.09 & 0.07 & 0.03 & 0.11 & 0.05 & 0.03 \\
$\mathbf{P S L}$ & Min & 10.71 & 0.39 & 0.19 & 0.86 & 0.55 & 0.25 \\
& Max & 17.28 & 0.60 & 0.28 & 1.25 & 0.73 & 0.36 \\
\hline \hline
\end{tabular}

The ANOVA procedure could not be directly applied to composite MOE data, because of the severe inequality of variances. A logarithmic transformation made the variance of the different groups more homogeneous. Two-way Analysis of Variance, executed using the transformed data, showed that variations both in load and in strand/layer orientation caused statistically significant differences in the compression MOE of LVL and PSL (see APPENDIX B.)

The compression MOE of LVL and PSL in the longitudinal $(x)$ direction is significantly higher than that of their raw material (yellow-poplar.) The slightly better performance of LVL was expected, because of some densification present in this composite. In PSL, strands are not aligned perfectly in the longitudinal direction, which reduces the MOE value, but the high level of densification improves the MOE significantly. The particular composite used in this study also includes $25 \%$ southern yellow pine material (see section 5.3.1), which is likely to have improved the MOE further. This explains why PSL performed better than solid yellow-poplar wood and LVL in terms of compression elasticity. 
In the other directions, the composites' MOE values are either comparable to or worse than those of yellow-poplar. This was unexpected, since the same tendencies outlined above should lead to higher MOE values in these directions.

\subsection{Composite geometry}

\subsubsection{Geometry of the raw materials}

Table 6.15 summarizes the thickness statistics of aspen, red oak and yellowpoplar veneer. Average veneer thickness was somewhat lower than the target peeling thickness of $3.2 \mathrm{~mm}$. The reason for this is shrinkage during the drying of the material. The magnitude of shrinkage depends on the specific gravity of wood (Siau 1995), which is different for the three species. This explains the difference between the mean thickness values.

Figure 6.19 shows the histograms created from the thickness measurements. The overlaid probability density functions were chosen based on the ranking provided by ExpertFit $^{\circledR}$. The figure also indicates the type and parameters of the chosen statistical

Table 6.15 - Summary statistics of the thickness of veneer sheets manufactured from the different species. (Thickness values are in $\mathrm{mm}$ )

\begin{tabular}{lccc}
\hline & Aspen & Red oak & Yellow-poplar \\
\hline Mean & $\mathbf{3 . 0 7}$ & $\mathbf{3 . 0 5}$ & $\mathbf{3 . 1 2}$ \\
S & 0.08 & 0.09 & 0.09 \\
Median & 3.07 & 3.05 & 3.12 \\
Minimum & 2.82 & 2.85 & 2.82 \\
Maximum & 3.25 & 3.28 & 3.28 \\
Skewness & -0.586 & 0.029 & -0.833 \\
Sample size & 80 & 84 & 84 \\
\hline \hline
\end{tabular}




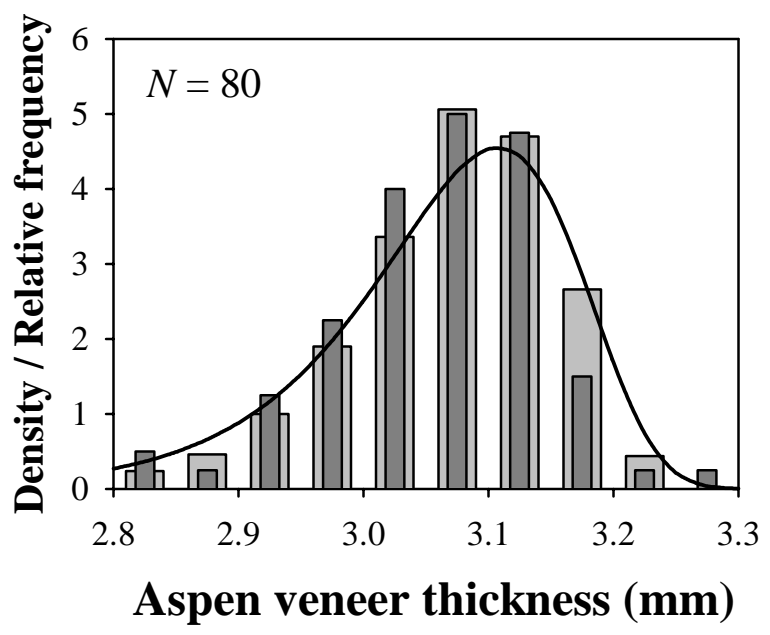

Probability density function:

Extreme Value type A

Parameters: $\mu=3.108$

$\sigma=0.069$

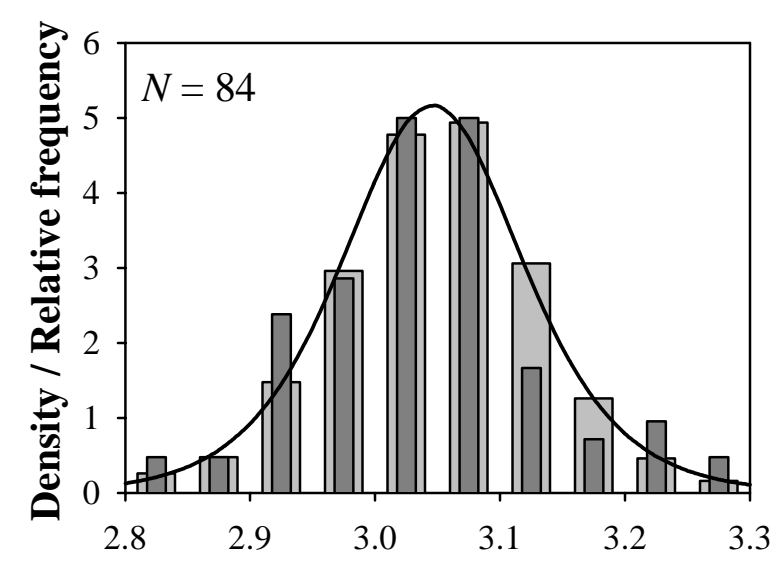

Red oak veneer thickness ( $\mathrm{mm})$

Probability density function:

Logistic

Parameters: $\mu=3.046$

$\sigma=0.048$

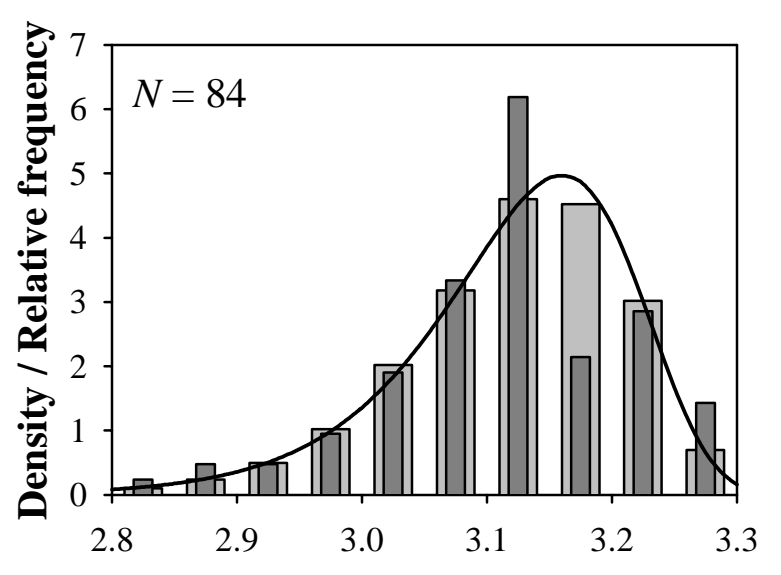

Yellow-poplar veneer thickness (mm)

Probability density function:

Two-parameter Weibull

Parameters: $\sigma=3.162$

$\alpha=42.761$

$\square$ Experimental distribution ( $N$ measurements)

Simulated distribution (1000 random deviates)

Probability density function

Figure 6.19 - Statistical distribution and probability density function of the veneer thickness for the three species 
distributions, and contains histograms created by simulating 1000 random deviates for each parameter. Visual evaluation confirmed the good performance of these distributions to describe the data, except for yellow-poplar veneer. In this species, thickness values concentrate in the vicinity of $3.12 \mathrm{~mm}$, while measurements are scarce around $3.17 \mathrm{~mm}$. There seems to be no theoretical reason behind this phenomenon, which is probably due to random variation in the data. It was concluded that the plotted Weibull density function represents the experimental values well, despite this anomaly.

\subsubsection{Geometric properties of LVL}

The thickness of the layers in LVL did not exhibit very high variation throughout the cross-section (Figure 6.20), although one-way ANOVA showed significant differences between the layer thicknesses. Tukey's multiple comparison test indicated statistically significant differences between the thickness of the two outside layers on both sides, and that of the remaining layers. This is in accordance with findings reported in the literature, that hot pressing causes more densification in the faces than in the core material of composites (Harless et al. 1987, Xu and Winistorfer 1996, Song and Ellis 1997.) Based on these results, layer thickness data was pooled into two groups: outside and core layer thickness. Table 6.16 provides the most important statistical parameters of the two groups, as well as those of overlap length and distance ( $\lambda$ and $\delta$ ) in LVL.

Figure 6.21 contains histograms generated from the experimentally measured database, together with fitted probability density functions that were deemed best for describing the actual values. The plotted functions and simulation results seem to follow the experimental data distributions well. 


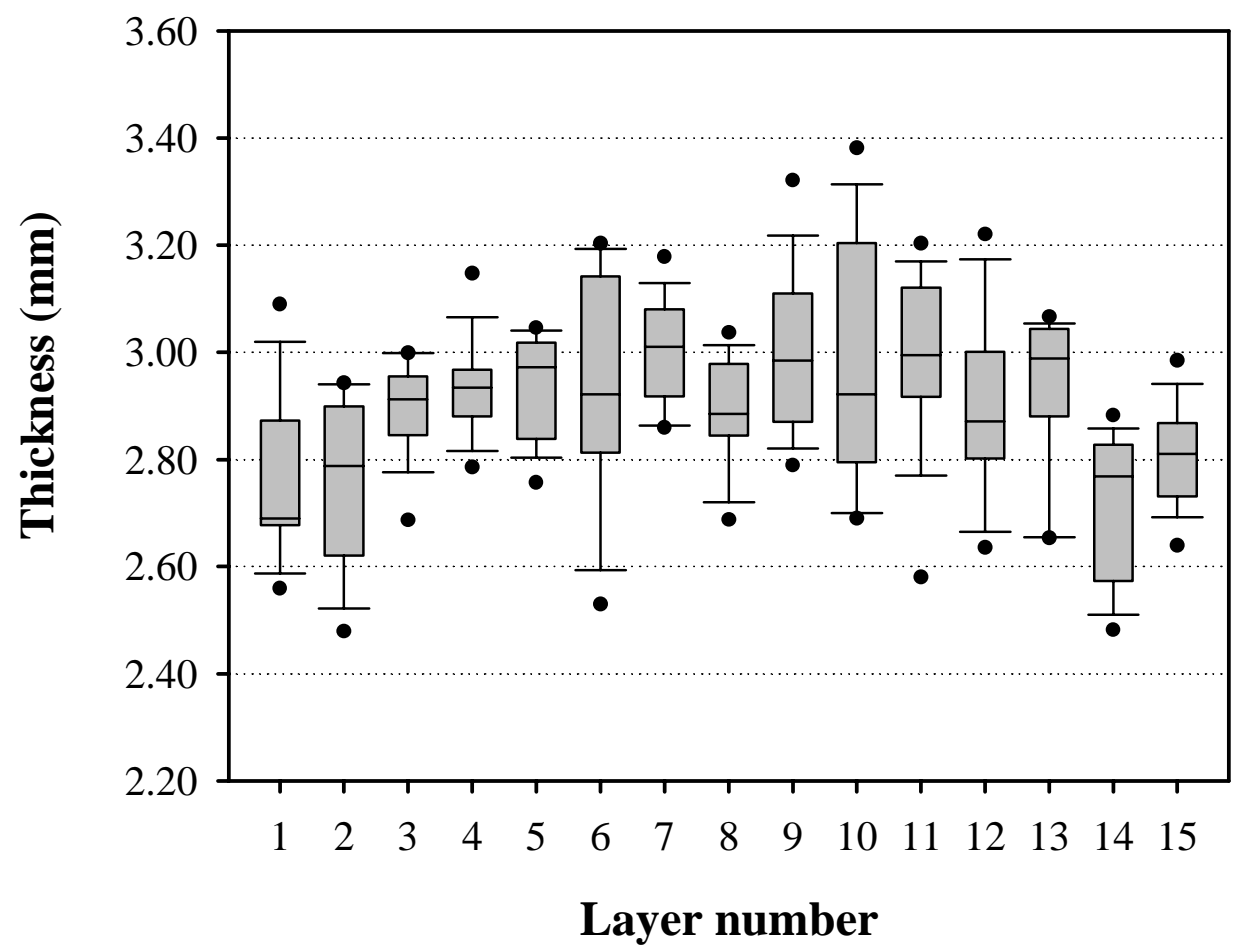

Figure 6.20 - Layer thickness distribution throughout the cross-section of LVL

Table 6.16 - Summary statistics of the geometric parameters of LVL

\begin{tabular}{lcccc}
\hline \hline & $\begin{array}{c}\text { Outside layer } \\
\text { thickness } \\
(\mathrm{mm})\end{array}$ & $\begin{array}{c}\text { Inside layer } \\
\text { thickness } \\
(\mathrm{mm})\end{array}$ & $\begin{array}{c}\text { Overlap } \\
\text { length } \\
(\lambda, \mathrm{mm})\end{array}$ & $\begin{array}{c}\text { Distance between } \\
\text { overlaps } \\
(\delta, \mathrm{mm})\end{array}$ \\
\hline Mean & $\mathbf{2 . 7 6}$ & $\mathbf{2 . 9 5}$ & $\mathbf{4 9 . 0 0}$ & $\mathbf{1 6 5 . 0 2}$ \\
$s$ & 0.14 & 0.15 & 14.24 & 14.93 \\
Median & 2.76 & 2.93 & 48.80 & 166.16 \\
Minimum & 2.48 & 2.53 & 21.71 & 93.19 \\
Maximum & 3.09 & 3.38 & 90.02 & 206.68 \\
Skewness & -0.08 & 0.01 & 0.31 & -0.80 \\
Sample size & 44 & 121 & 148 & 145 \\
\hline \hline
\end{tabular}




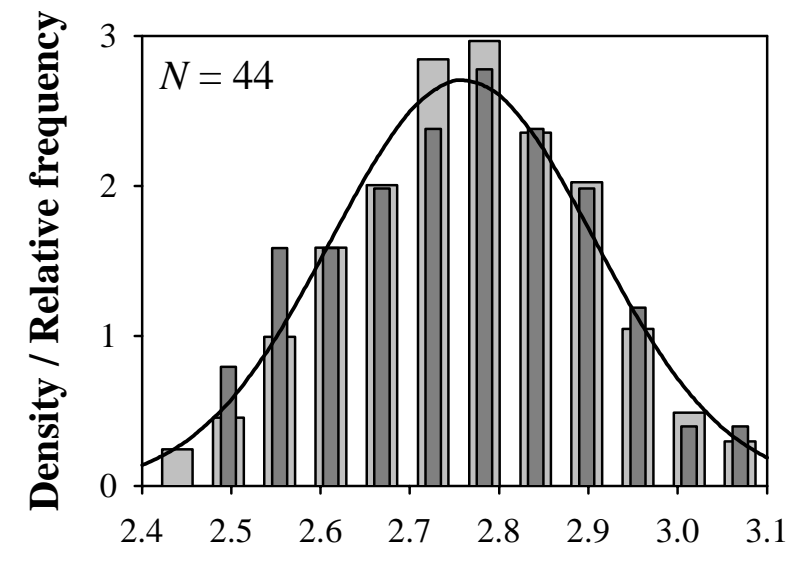

Outside layer thickness ( $\mathbf{m m}$ )

Probability density function:

Normal

$$
\text { Parameters: } \begin{aligned}
\mu & =2.76 \\
\sigma & =0.14
\end{aligned}
$$

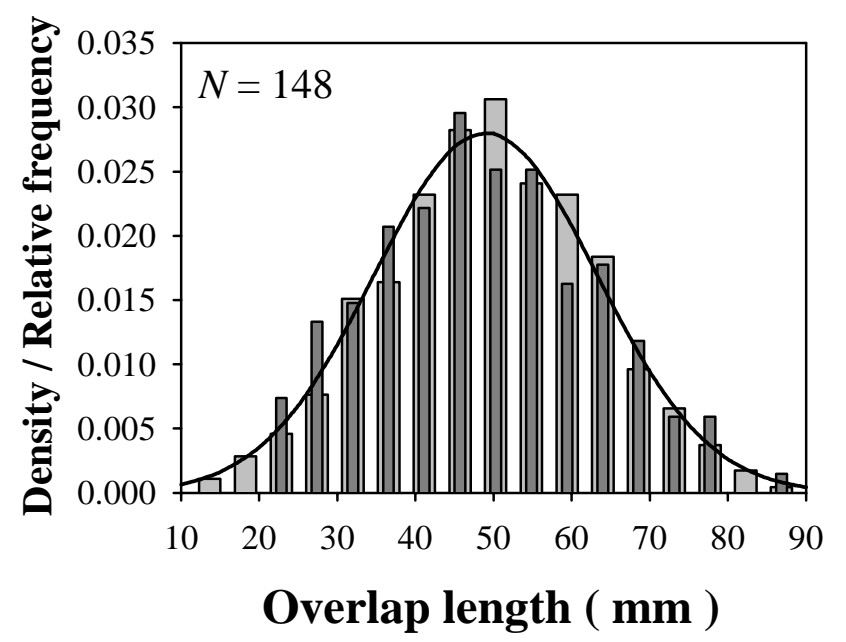

Probability density function:

Normal

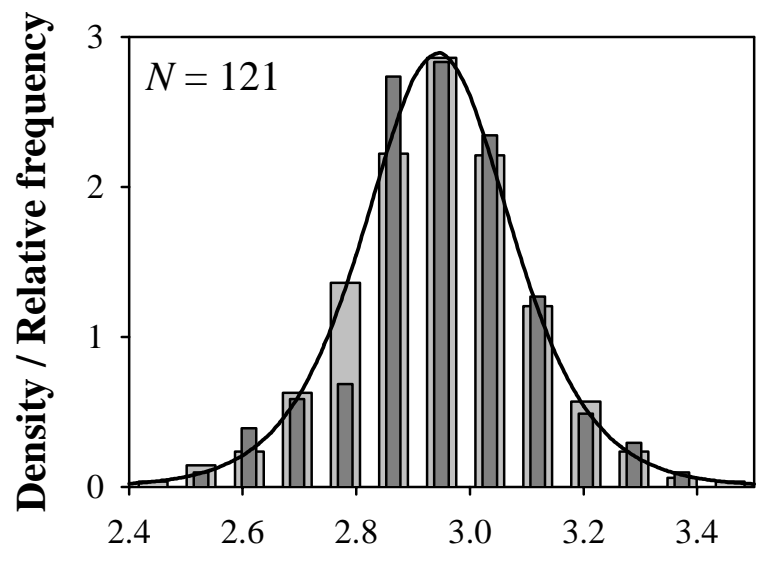

\section{Core layer thickness ( $\mathbf{m m}$ )}

Probability density function:

Logistic

Parameters: $\mu=2.94$

$$
\sigma=0.09
$$

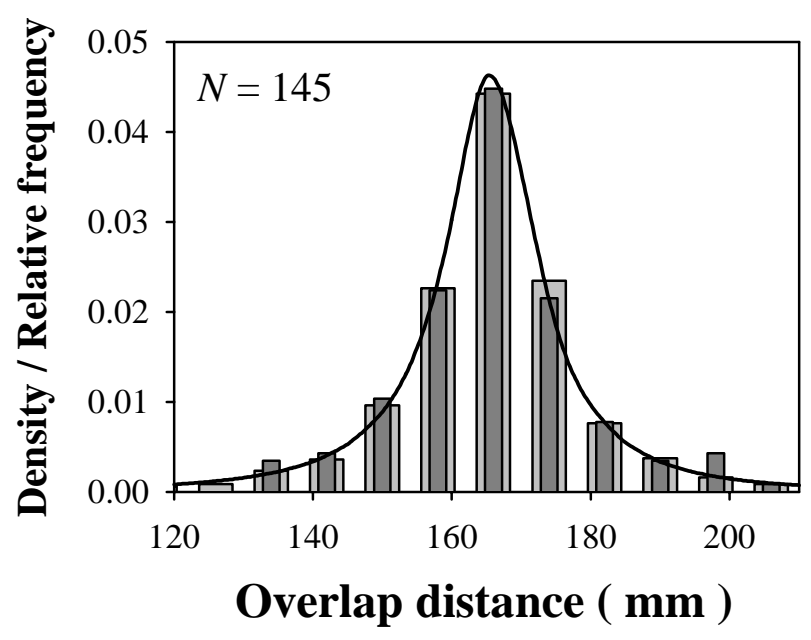

Probability density function:

Johnson SU

Parameters: $\begin{aligned} \mu & =165.83 & \alpha_{1}=0.053 \\ \sigma & =7.03 & \alpha_{2}=0.823\end{aligned}$

Figure 6.21 - Statistical distribution and probability density function of the geometric parameters of LVL 


\subsubsection{Geometric properties of PSL.}

Table 6.17 summarizes the statistical parameters of the geometric properties of PSL. The sample size of strand number and average strand thickness determination was relatively low, due to the difficulties involved in determining these parameters. The mean values of strand angle and strand orientation $(\alpha$ and $\beta$ ) were slightly different from the expected value of zero. This might be due to random error, but could also be the result of systematic deviations introduced during manufacture.

Figure 6.22 comprises the experimental histograms and the overlaid best fit probability density functions. The normal distribution was found to work well for every geometric parameter of PSL, except for $u$, where the function does not fit experimental data well. This seemingly bad fit is a result of the very limited sample size of the strand number determination. Because of the low number of samples for this parameter, it is

Table 6.17 - Summary statistics of the geometric parameters of PSL

\begin{tabular}{lcccc}
\hline \hline & $\begin{array}{c}\text { Strands/in } \\
(u)\end{array}$ & $\begin{array}{c}\text { Strand } \\
\text { thickness } \\
(t, \mathrm{~mm})\end{array}$ & $\begin{array}{c}\text { Strand angle } \\
\left(\alpha^{\circ}\right)\end{array}$ & $\begin{array}{c}\text { Strand } \\
\text { deviation } \\
\left(\beta^{\circ}\right)\end{array}$ \\
\hline Mean & $\mathbf{1 1 . 6 4}$ & $\mathbf{2 . 1 3}$ & $\mathbf{0 . 0 3}$ & $\mathbf{2 . 9 9}$ \\
$s$ & 0.36 & 0.37 & 4.67 & 14.71 \\
Median & 11.65 & 2.15 & 0.00 & 4.00 \\
Minimum & 10.81 & 1.38 & -12.00 & -45.00 \\
Maximum & 12.06 & 2.78 & 20.00 & 45.00 \\
Skewness & -1.34 & -0.14 & 0.16 & 0.45 \\
Sample size & 10 & 30 & 300 & 120 \\
\hline \hline
\end{tabular}




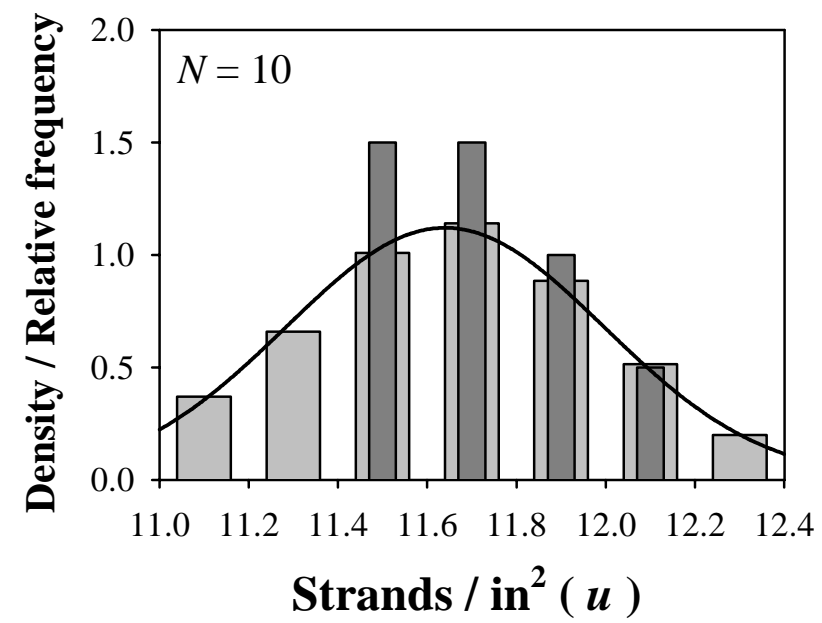

Probability density function:

Normal

Parameters: $\mu=11.640$

$\sigma=0.356$

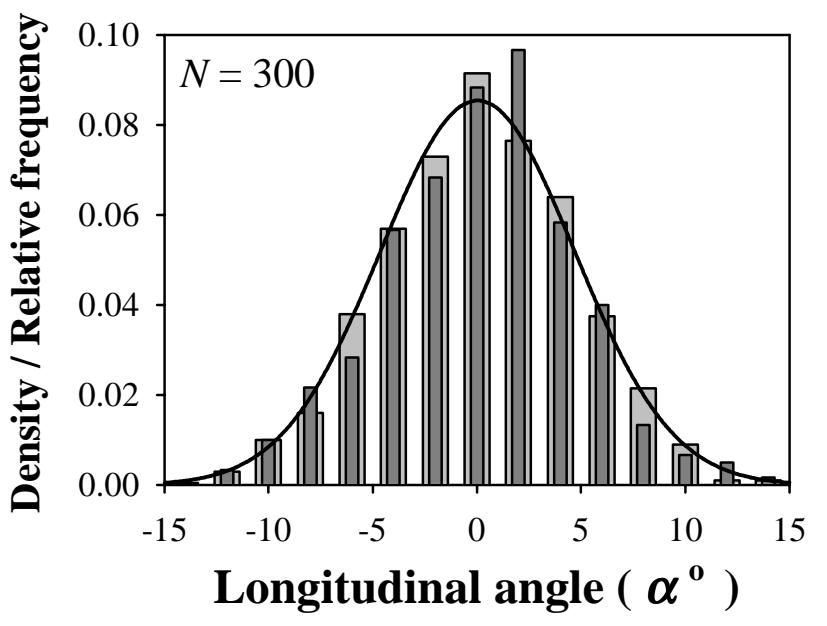

Probability density function:

Normal

Parameters: $\mu=0.030$

$\sigma=4.668$

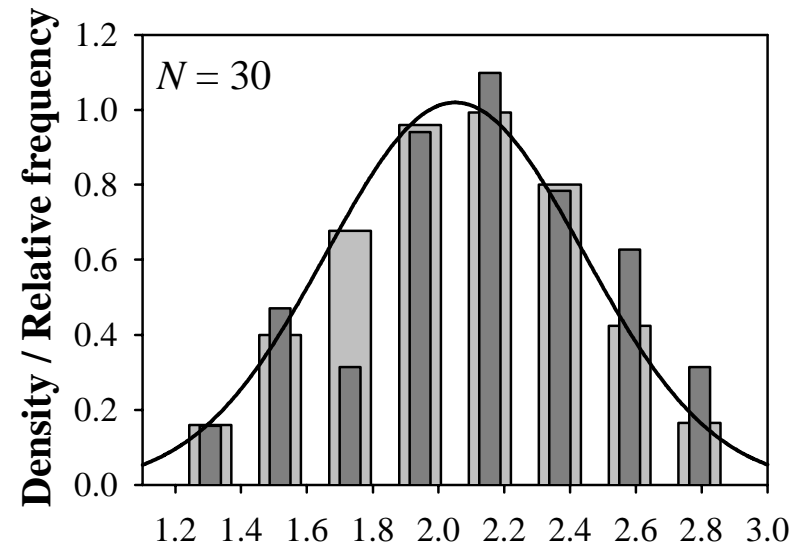

Strand thickness $(\boldsymbol{t} ; \mathbf{~ m m})$

Probability density function:

Normal

Parameters: $\mu=2.0506$

$\sigma=0.3911$

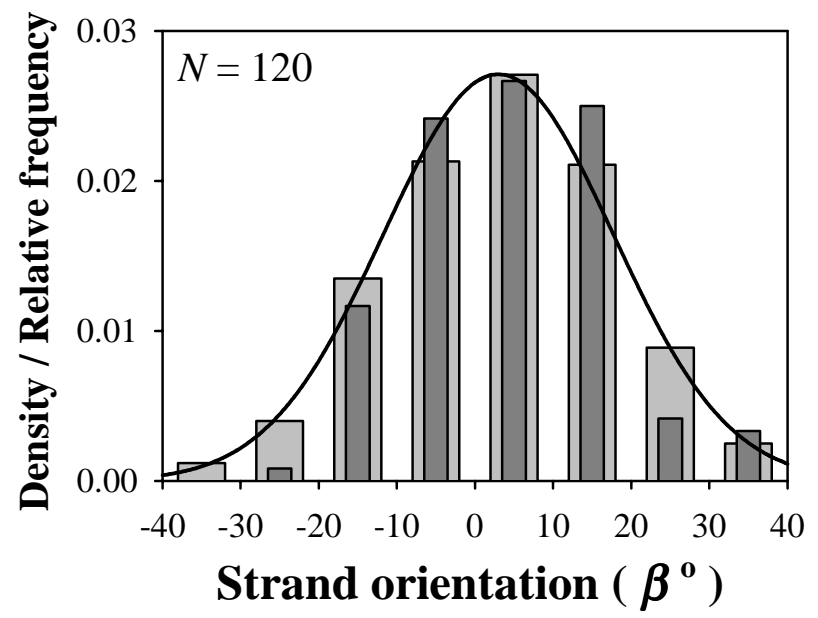

Probability density function:

Normal

Parameters: $\mu=2.991$

$\sigma=14.709$

Figure 6.22 - Statistical distribution and probability density function of the geometric parameters of PSL 
difficult to determine if a particular distribution describes experimental values adequately. The normal distribution was assumed to give a good representation of the number strands per in $^{2}$. Histograms created from 1000 simulated random deviates for each parameter agree with experimental distributions reasonably well, except for that of $u$.

\subsubsection{Probability density functions}

Some of the probability density functions used in the previous sections are used frequently to describe the distributions of stochastic variables (e.g. normal or Weibull distribution.) Others, like the Johnson SU or the Extreme Value distributions are less familiar. Interested readers can find further information concerning these distributions in Law and Kelton (1993) or Law and Vincent (1999). 


\section{Model DeVELOPMENT AND VALIDATION}

This chapter describes the creation of four separate models for the simulation of bending and orthotropic compression MOE of LVL and PSL, and their verification against the experimental data in sections 6.3.2 and 6.3.4. The models are based on principles of static simulation (Law and Kelton 1993), and deterministic and stochastic variables originated almost exclusively from the experimental results provided in chapter 6 .

\subsection{Model development}

Simulating the elastic properties of LVL and PSL consists of generating a virtual beam section that contains several elements, each with certain geometric and orthotropic elastic properties. The geometry and elasticity of the constituents are governed by deterministic and probabilistic input variables.

\subsubsection{Simplifying assumptions}

To simplify the simulation task, composites are treated as prismatic beams; that is, their cross-section does not change throughout the length of the beam. Geometrically, constituents are represented by their cross-sections. Further simplifications used in the models include the following items:

- Veneers used for layers and strands are peeled perfectly tangential to the annual ring (i.e., their plane is $L T$ );

- A continuous glueline provides perfect adhesion between the layers or strands; 
- The applied glue does not alter the MOE of the layers or strands significantly;

- The grain orientation of an LVL layer is always parallel with the longitudinal axis of the beam; the grain orientation of a PSL strand is always parallel with the longitudinal axis of the strand;

- The layup is random; the MOE values of the layers or strands are independent of their position;

- The thickness of a layer in LVL is constant; irregularities, such as crushed-lap joints that connect two veneer sheets in the same layer, are disregarded;

- PSL strand cross-sections are rectangular in shape; strands are not bent or distorted;

- $\quad$ PSL strand width is constant $(25 \mathrm{~mm})$;

- The densification of a PSL strand is independent from its position within the billet.

None of the above assumptions are fully justified in reality. There is reason to believe, however, that these simplifications do not bias simulation results seriously, while reducing the programming complexity and execution times considerably.

\subsubsection{Simulation of the constituents' geometric parameters}

Simulating the geometric properties of LVL included the following steps:

- Establishing the number of layers. This is a deterministic variable that depends on the particular material being simulated. In the present case, the number of layers was 15 .

- Assigning original and final thickness values to each layer from their respective probability distributions.

- Calculating cross-section $\left(A_{i}\right)$ and the $2^{\text {nd }}$ order moment of inertia around the symmetry axis of the composite $\left(I_{i}\right)$, for each layer. (The width of the beams can be any value; because the model assumes no horizontal variation of any kind, it does not impact simulation results.) 
The simulation of the cross-sectional geometry of PSL involved the following procedures:

- Choosing the $y$ and $z$ cross-sectional dimensions;

- Simulating the number of strands. In PSL, the number of constituents is a random variable, established by generating the number of strands per $\operatorname{in}^{2}(u)$, and multiplying this number by the cross-sectional area;

- Assigning original and the projected final thickness $\left(t_{o}\right.$ and $\left.t\right)$, longitudinal angle $(\alpha)$ and cross-sectional orientation $(\beta)$ values to each strand. (The model neglects the deviation of the strand's longitudinal axis from the $x y$ plane, which is small in reality.) The width of the strands is $25 \mathrm{~mm}$, except for one strand, which has a smaller width. This strand reflects the fractional part of the strand number. For the calculation of the projected strand width and the actual strand thickness see APPENDIX $\boldsymbol{C}$;

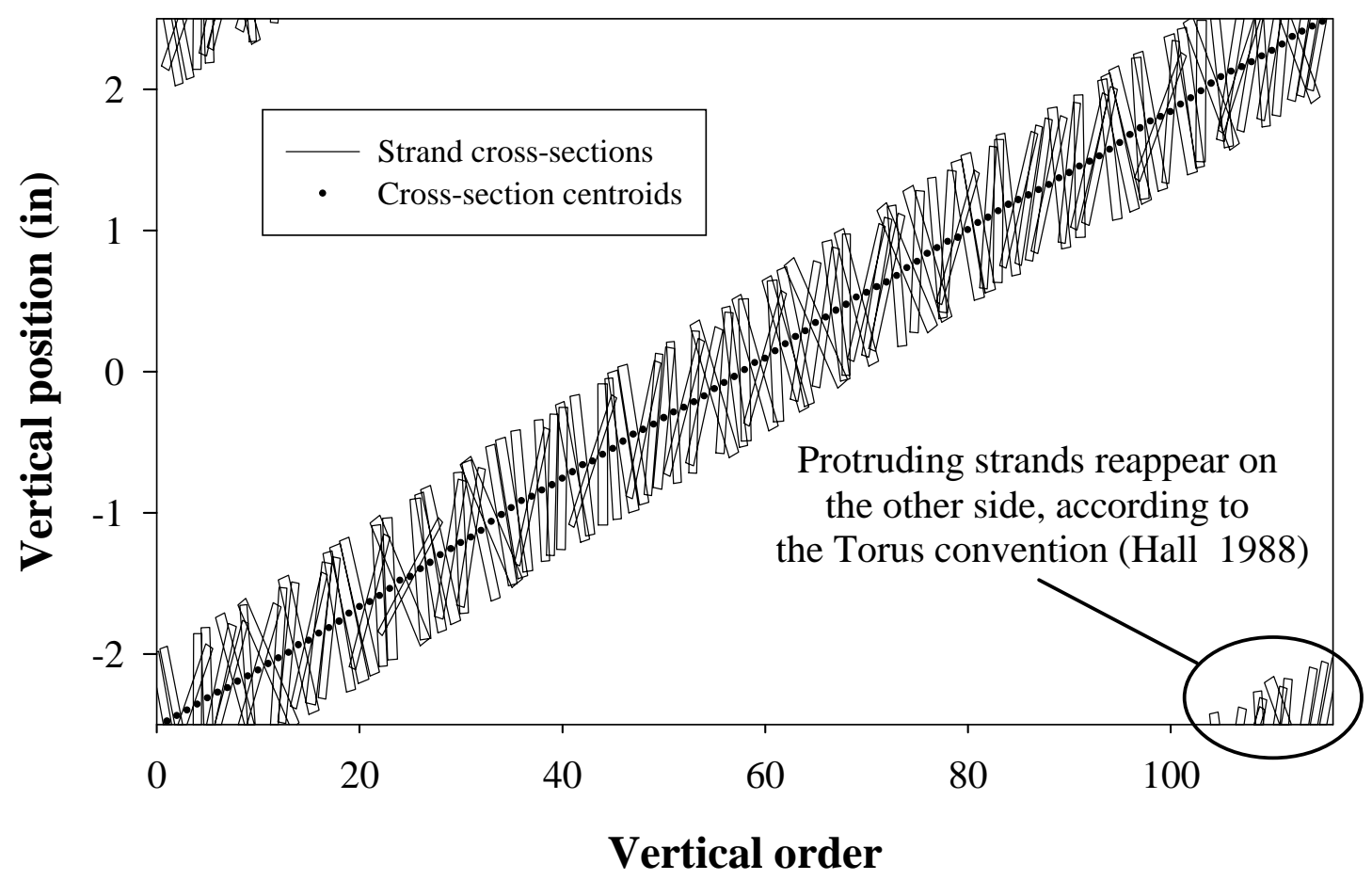

Figure 7.1 - The systematic arrangement of the strands in PSL 
- Arranging strands in a random order in both the $y$ and the $z$ directions. To achieve an even coverage, strand centroids were distributed uniformly in a systematic way according to this order, rather than randomly (Figure 7.1);

- Calculating $I_{i}$ both flatwise and edgewise, for each constituent, as well as their crosssectional area $\left(A_{i}\right)$. Strands that protrude beyond the boundary of the beam cross section are handled according to the torus convention (Hall 1988). For an explanation of this principle, see Figure 7.1.

For both composite types, $I_{i}$ values are calculated around the neutral axis of the beam. The neutral axis in a homogenous material goes through the centroid of the crosssection. If the compression and tension Moduli of Elasticity are different (as they are in wood), the neutral axis might shift from this position. The simulation model assumes that the neutral axis is same as the symmetry axis of the cross-section. This reduces simulation times significantly, introducing only minor inaccuracies in the results.

The model breaks $I_{i}$ values of each strand or layer into two components; $I_{C}$ and $I_{T}$, the moment of inertia of the portion of the cross-section subjected to compression and tension, respectively. If a strand or layer is located entirely in the compression or tension zone (strands $a$ and $b$ on Figure 7.2, respectively), its $I_{T}$ or $I_{C}$ will be equal to zero, respectively. If the cross-section of a constituent crosses the neutral axis (strand $c$ ) or - in PSL - protrudes outside of the beam cross section (strand $d$ ), both its $I_{T}$ and $I_{C}$ will be different from zero. 


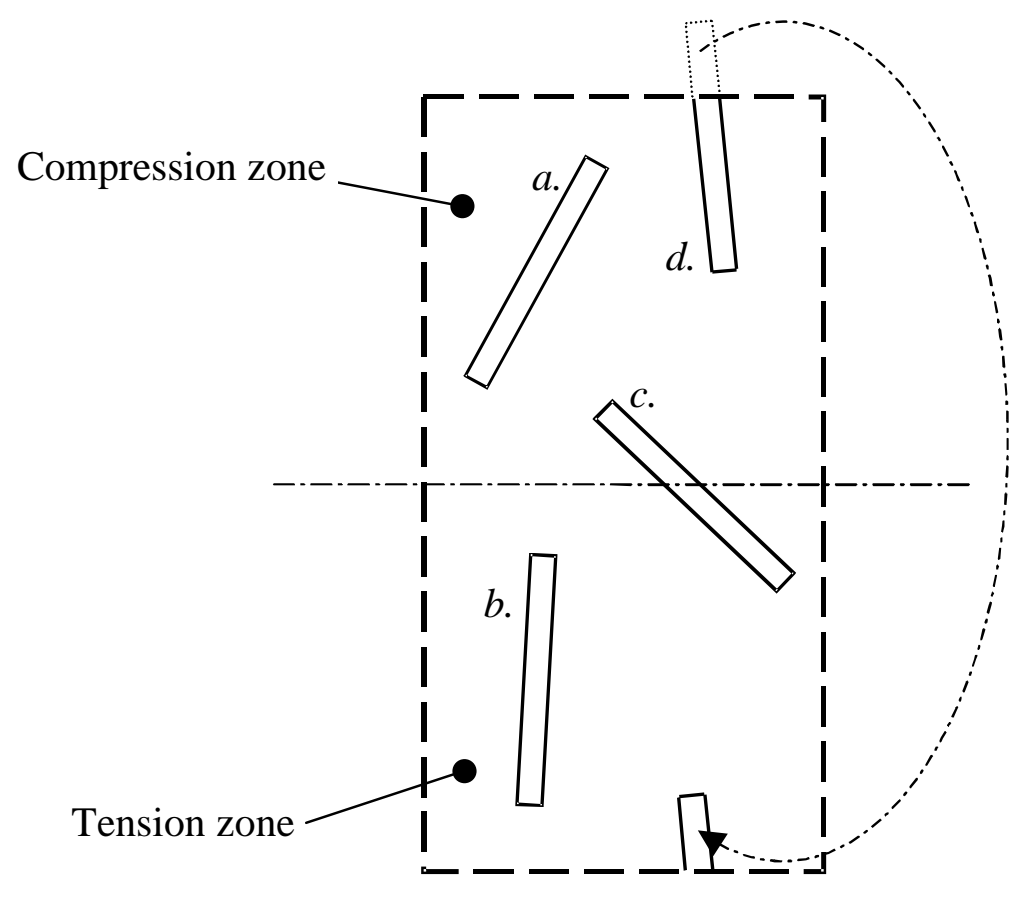

Figure 7.2 - Strands situated at various positions in a PSL cross-section

\subsubsection{Simulation of the composites' geometric and physical properties}

To validate the simulated cross-sectional structure, certain geometric properties of the composites were calculated from the modeled parameters. In LVL, this included the panel thickness, which is equal to the combined thickness of the layers. For PSL, the combined cross-sectional area $\left(\Sigma A_{i}\right)$ and combined flatwise and edgewise $2^{\text {nd }}$ order moment of inertia $\left(\Sigma I_{i \text { Flat }}\right.$ and $\left.\Sigma I_{i E d g e}\right)$ of the strands were calculated.

Simulation of the composites' density provided another opportunity to validate the simulated geometry. This involved generating original density values for the constituents, using the experimental data shown in Table 6.1. Because the limited sample size of the density measurements prevented reliable probability density function determination, normal distribution was assumed. Using the simulated density values, the 
weight of every constituent was calculated for a $25 \mathrm{~mm}$ (1 in) long beam section. The ratio of the combined strand weight and the volume of the beam section equals to the density of the beam.

\subsubsection{Simulation of bending MOE}

Equation 4.18 requires the simulation of the modulus of elasticity $\left(E_{i}\right)$ for each constituent. Since the MOE in tension can be very different from that in compression, each LVL layer or PSL strand is assigned both a compression and a tension MOE value.

In LVL, the grain direction of the layers always runs parallel with the longitudinal axis of the beam; that is, the direction in which compression and tension stresses act. Compression and tension MOE can be simulated using the statistical distribution of the longitudinal $\mathrm{MOE}\left(E_{L}\right)$. PSL strands are running at a (typically small) inclined angle to the longitudinal axis of the beam, and orthotropic theories can estimate their compression and tensile elastic parameters.

Compression MOE can be simulated by supplying Equation 4.10 or Equation 4.12 with the grain- and ring orientation of the load relative to the individual strands, as well as the simulated elastic constants $\left(E_{L}, E_{R}, E_{T}, E_{45^{\circ}}^{0^{\circ}}, E_{45^{\circ}}^{90^{\circ}}, E_{90^{\circ}}^{45^{\circ}}\right)$ that the equations require. (Both of these equations are applicable for MOE, as well as compression strength.) Tensile MOE is generated in two steps. First, the model simulates the dynamic MOE using the combination provided in Equation 6.3, based on the strand's grain angle $(\varphi=\alpha)$ and the simulated dynamic elastic parameters at $0^{\circ}, 45^{\circ}$ and $90^{\circ}$ grain angles. Following that, it converts the simulated value into tensile MOE using the second order regressive relationship between the dynamic and static MOE (Figure 6.13 b.) 
Because the sample size of compression and dynamic MOE measurements was not large enough to allow a reliable statistical distribution determination, the elastic constants were assumed to be normally distributed, and simulated accordingly. The model does not account for the possible correlation between the MOE values of wood in the different anatomical directions. This correlation probably exists, but, since the variation of MOE is very small in the non-longitudinal directions, its effect is likely to be negligible.

The compression and tensile MOE of the constituents, as simulated above, reflect their elastic properties before the composite goes through the consolidation process. While in the press, the layers and strands experience densification, which, as shown in section 6.2, modifies their MOE values. The model simulates the MOE increase of each constituent due to densification, using the experimental relationships shown on Figure 6.14, modified with the factor calculated in section 6.2.2.

Using the simulated densified compression and tension MOE, along with the $I_{T}$ and $I_{C}$ values calculated for each constituent, laminate theory can be written in the following form:

$$
E=\frac{\Sigma\left(E_{T i} I_{T i}+E_{C i} I_{C i}\right)}{I}
$$

where: $E_{T i}, E_{C i}$ - tensile and compression MOE of the $i^{\text {th }}$ strand or layer, respectively;

$I_{T i}, I_{C i} \quad$ - moment of inertia of the constituent's portion subjected to tension and compression, respectively;

E, $I \quad$ - effective bending MOE and moment of inertia of the beam.

The above principles can be used to create models that can simulate the bending MOE of LVL and PSL beams in both edgewise and flatwise application. 


\subsubsection{Simulation of the orthotropic compression MOE}

Simulation of compression MOE in a certain direction is a similar process to modeling bending MOE. The differences are, as follows:

1. The model assigns only a compression MOE to every constituent. $E_{C}$ is calculated using Equation 4.10 or 4.12. The grain and ring angles for each component relative to the compression load are calculated from $\varphi^{\prime}$ and $\theta^{\prime}$ (load orienation and strand/layer orientation, as defined in section 5.3), and, in the case of PSL, $\alpha$ and $\beta$. (For the details of this calculation, see APPENDIX C.)

2. Instead of the moment of inertia, the volume of each strand or layer is calculated, as the product of its cross-sectional area and the length of the simulated beam section.

3. Equation 4.19 is used to calculate the compression $\mathrm{MOE}$ of the simulated composite in the given direction.

\subsubsection{Methods and procedures}

The FORTRAN 90 programming environment was used to create four simulation models, based on the principles described in the preceding sections. The source code includes four programs, as well as several different functions to generate random data from different distributions, simulate the orthotropic tensile and compression MOE along with the densification effect, etc. A module stores the species-specific input parameters. The simulation can be easily extended to include new species by adding their parameters to this module. APPENDIX $\boldsymbol{E}$ contains the source code and documentation of the programs, functions, and the module comprising species-specific information. 
Validation of the bending models involved simulating twenty yellow poplar LVL and PSL beams, and calculating their MOE both in flatwise and edgewise orientations. The cross-sectional dimensions of the simulated PSL beams were the same as the target dimensions of the experimentally measured specimens. The Monte Carlo simulation was repeated twenty times, using a different random seed for each of the 400 beams. To ascertain complete independence of the simulated beams, the stream of random integers, used as seeds in these simulations, were provided by the SigmaPlot ${ }^{\circledR}$ scientific graphing program, rather than FORTRAN. Compression model validation was similar to the above procedure, but it included 20 groups of only 10 simulated specimens in the six experimentally investigated orientations.

Some further practical considerations that were used in creating the final models included the following points:

- Although the orthotropic tensor theory (Equation 4.10) provided best fit to the orthotropic compression MOE of hardwoods by $\mathrm{r}^{2}$ analysis, in the case of yellowpoplar's it estimates unrealistically high MOE when both grain and ring angles are at low levels (Figure 6.7.) These orientations are especially important in PSL strands, when the beam is subjected to bending or longitudinal compression. Thus, instead of the equation based on orthotropic tensor theory, the three-dimensional Hankinson formula (Equations 4.11 and 4.12) was used in the model.

- Most of the probability density functions that describe the mechanical and geometric parameters are unbounded. This might result in generating unrealistically low simulation results (e.g. negative MOE, or final thickness values that are higher than the original thickness of the constituents.) Certain safeguards are built in the routines and functions to exclude such values. 
- In PSL, the number of strands in a cross-section and the thickness of the strands are not independent variables. Assessing the correlation structure, however, would have required immense experimental work. The model disregards the relationship between these variables, which can result in unrealistically high or low compound crosssections of the strands - sometimes exceeding the cross-section of the beam. This should clearly not be allowed. However, discarding beams with such cross-sections could bias the average strand number per unit cross section, as well as the average strand thickness. One solution for this problem is to reject beams with too low, as well as too high combined strand cross-section. From trial simulations it was established that eliminating beams with $\Sigma A_{i}$ below 0.95 times the beam cross section maintains the average values of these parameters. If $\Sigma A_{i}$ is smaller than $0.95 \mathrm{~A}$ or higher than $A$, the programs re-simulate the beam.

\subsection{Model validation}

Model validation happened through the comparison of the simulated and actual geometric, physical and mechanical parameters of the composites. Experimental LVL beams consist of $100 \%$ yellow-poplar material, and the model was set up accordingly. PSL contains $25 \%$ southern yellow pine material. Unfortunately, input parameters for this species were unavailable. The simulation was based on $100 \%$ yellow-poplar material, which introduced some inaccuracy in the results. The cross-sectional dimensions of the simulated PSL beams were 140 by $75 \mathrm{~mm}$ ( 5.5 by 3 inches), the same as the target crosssection of the experimental beams used for model validation (section 5.3.2.)

The purpose of standard statistical tests is typically to show that two groups of numbers are statistically different, and proof of the opposite is hard to achieve. Even the so-called goodness-of-fit type tests might fail to show significant differences if a small 
number of specimens is used, while rejecting the assumption of equality at large sample sizes, even if the differences are minute. Because simulated and experimental results are never exactly the same, good fit shown by these tests is often due to lack of power, rather than an indication of actual agreement between the two distributions (Law and Kelton 1993.)

The present validation study does not seek to prove that simulated and experimental results are exactly the same. The goal is to show if a reasonable agreement exists between the two groups of data, based on engineering judgement, rather than statistical proof.

\subsubsection{Geometric and physical properties of LVL and PSL}

Table 7.1 comprises the simulation results of the geometric and physical properties of the composites. Results reported here are average values of the mean, standard deviation, minimum, maximum and skewness calculated from the twenty Monte Carlo simulations, containing twenty beams each. Individual statistics for each simulation run are provided in APPENDIX $\boldsymbol{F}$.

Table 7.1 - Simulated geometric and physical properties of LVL and PSL (experimental LVL thickness statistics are also included)

\begin{tabular}{lrrrrrrr}
\hline \hline & \multicolumn{3}{c}{ LVL } & \multicolumn{4}{c}{ PSL } \\
& $\begin{array}{c}\boldsymbol{T}_{\text {sim }} \\
(\mathbf{m m})\end{array}$ & $\begin{array}{c}\boldsymbol{T}_{\text {exp }} \\
(\mathbf{m m})\end{array}$ & $\begin{array}{c}\boldsymbol{\rho} \\
\left(\mathbf{k g} / \mathbf{m}^{\mathbf{3}}\right)\end{array}$ & $\begin{array}{c}\Sigma \boldsymbol{A}_{\boldsymbol{i}} \\
\left(\mathbf{c m}^{\mathbf{2}}\right)\end{array}$ & $\begin{array}{c}\Sigma \boldsymbol{I}_{\boldsymbol{i} \text { Edge }} \\
\left(\mathbf{c m}^{\mathbf{4}}\right)\end{array}$ & $\begin{array}{c}\Sigma \boldsymbol{I}_{\boldsymbol{i} \text { Flat }} \\
\left(\mathbf{c m}^{\mathbf{4}}\right)\end{array}$ & \multicolumn{1}{c}{$\begin{array}{c}\boldsymbol{\rho} \\
\left(\mathbf{k g} / \mathbf{m}^{\mathbf{3}}\right)\end{array}$} \\
\hline Mean & $\mathbf{4 2 . 9 6}$ & $\mathbf{4 3 . 5 6}$ & $\mathbf{5 1 1}$ & $\mathbf{1 0 3 . 7}$ & $\mathbf{1 6 8 7}$ & $\mathbf{4 9 8 . 3}$ & $\mathbf{6 7 3}$ \\
STD & 0.50 & 0.15 & 10 & 1.4 & 23 & 6.8 & 11 \\
Min & 42.05 & 43.13 & 492 & 101.4 & 1649 & 486.9 & 652 \\
Max & 43.85 & 43.97 & 530 & 106.1 & 1726 & 509.7 & 694 \\
Skewness & 0.005 & 0.013 & -0.100 & 0.029 & 0.029 & 0.001 & -0.037 \\
\hline \hline
\end{tabular}


In LVL, simulated thickness and density is somewhat different from the experimental values. (Table 7.1 includes the experimental thickness statistics and Table 6.10 contains the measured density values.) Simulated thickness values are somewhat smaller in average, but include nearly the whole range of the experimental values. Simulated density is significantly lower than the experimentally measured values. This might be caused by several different factors, such as the dry material content of the glue, high-density knots in the veneers and the presence of the crushed-lap joints. The model does not account for the effect of these factors.

The simulated combined cross-sectional area of PSL is within the range of $95 \%$ to $100 \%$ of the cross-section of the beam $\left(106.5 \mathrm{~cm}^{2}\right)$, according to the restrictions described in section 7.1.6. The average cross-sectional area was $97.4 \%$ of the beam crosssection. The relationship between the combined edgewise and flatwise $2^{\text {nd }}$ order moment of inertia of the strands and the respective $I$ values of the beam $\left(1731\right.$ and $\left.515.1 \mathrm{~cm}^{4}\right)$, showed a similar relationship. The combined cross-sectional area and moment of inertia of the strands are usually slightly smaller than those of the beam, because PSL contains some void volume, due to the slightly imperfect packing of the strands. Visual appraisal showed that the simulated $95 \sim 100 \%$ coverage is reasonable for PSL. The simulated density, which shows excellent agreement with actual values in Table 6.10, further affirms the accuracy of the simulated geometric structure.

Figure 7.3 demonstrates the similarity of the simulated and experimental crosssections visually. The simulated LVL cross-section (a) contains 15 layers with thicknesses generated from the appropriate statistical distributions, and is compared to an 

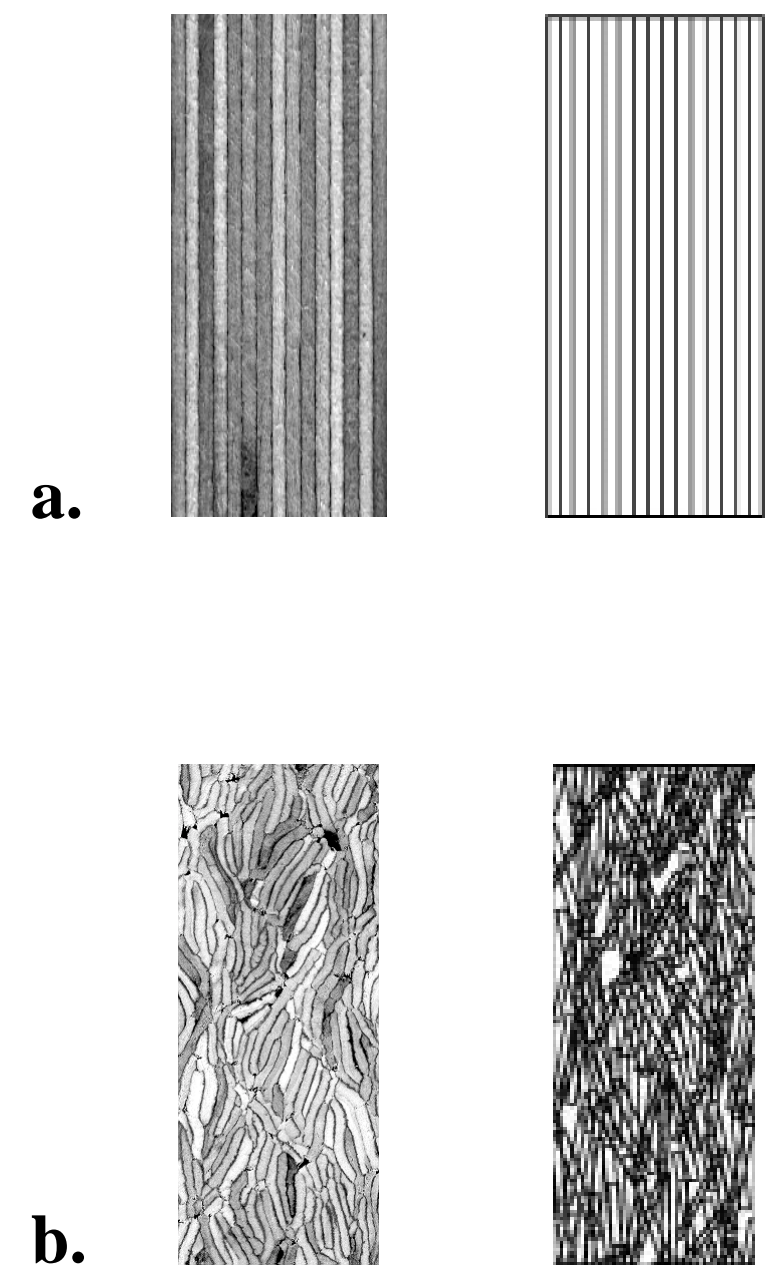

Figure 7.3 - Experimental and simulated cross-sections of LVL (a) and PSL (b) 
overlap-free experimental cross-section. The PSL cross-section (b) was created using the principles described in section 7.1.2, except for the horizontal distribution of the strands, which is optimized for the visual demonstration. Notice the black areas representing holes in the actual PSL cross-section.

\subsubsection{Validation of the bending MOE simulation}

Table 7.2 contains the statistical summary of the bending MOE simulation results for LVL and PSL. Values are, again, averages of twenty simulation runs, detailed in APPENDIX $\boldsymbol{F}$. Simulated values can be compared to the experimental bending MOE of LVL and PSL in Table 6.11. Figure 7.4 demonstrates the relationship between the simulated and experimental mean bending MOE values.

The simulated bending MOE of LVL is somewhat lower than the experimental values. The differences, however, are not very pronounced, especially in flatwise application, where, for example, simulation nr. 5 (Table E.4) provided a mean bending MOE very similar to the measured average value. Simulated beams were slightly stronger in flatwise than in edgewise application, which also follows the actual pattern. It appears that, despite minor differences, the simulation model estimates the MOE of LVL with reasonable accuracy.

The simulation results for the bending MOE of PSL are significantly higher than the experimental values reported in Table 6.11. There are several plausible explanations for this inaccuracy. PSL strands go through a considerable amount of distortion and disintegration during the hot pressing procedure, which might reduce their tension and 
Table 7.2 - Simulated edgewise and flatwise bending MOE of LVL and PSL

\begin{tabular}{lccrc}
\hline & \multicolumn{2}{c}{ LVL } & \multicolumn{2}{c}{ PSL } \\
& $\begin{array}{c}\mathbf{E}_{\text {edge }} \\
(\mathrm{GPa})\end{array}$ & $\begin{array}{c}\mathbf{E}_{\text {flat }} \\
(\mathrm{GPa})\end{array}$ & $\begin{array}{c}\mathbf{E}_{\text {edge }} \\
(\mathrm{GPa})\end{array}$ & $\begin{array}{c}\mathbf{E}_{\text {flat }} \\
(\mathrm{GPa})\end{array}$ \\
\hline Mean & $\mathbf{1 2 . 7 0}$ & $\mathbf{1 2 . 9 1}$ & $\mathbf{1 4 . 5 4}$ & $\mathbf{1 4 . 4 6}$ \\
S & 0.41 & 0.79 & 0.40 & 0.34 \\
Min & 11.95 & 11.40 & 13.78 & 13.83 \\
Max & 13.49 & 14.46 & 15.29 & 15.10 \\
Skewness & 0.063 & 0.100 & -0.052 & 0.041 \\
\hline \hline
\end{tabular}

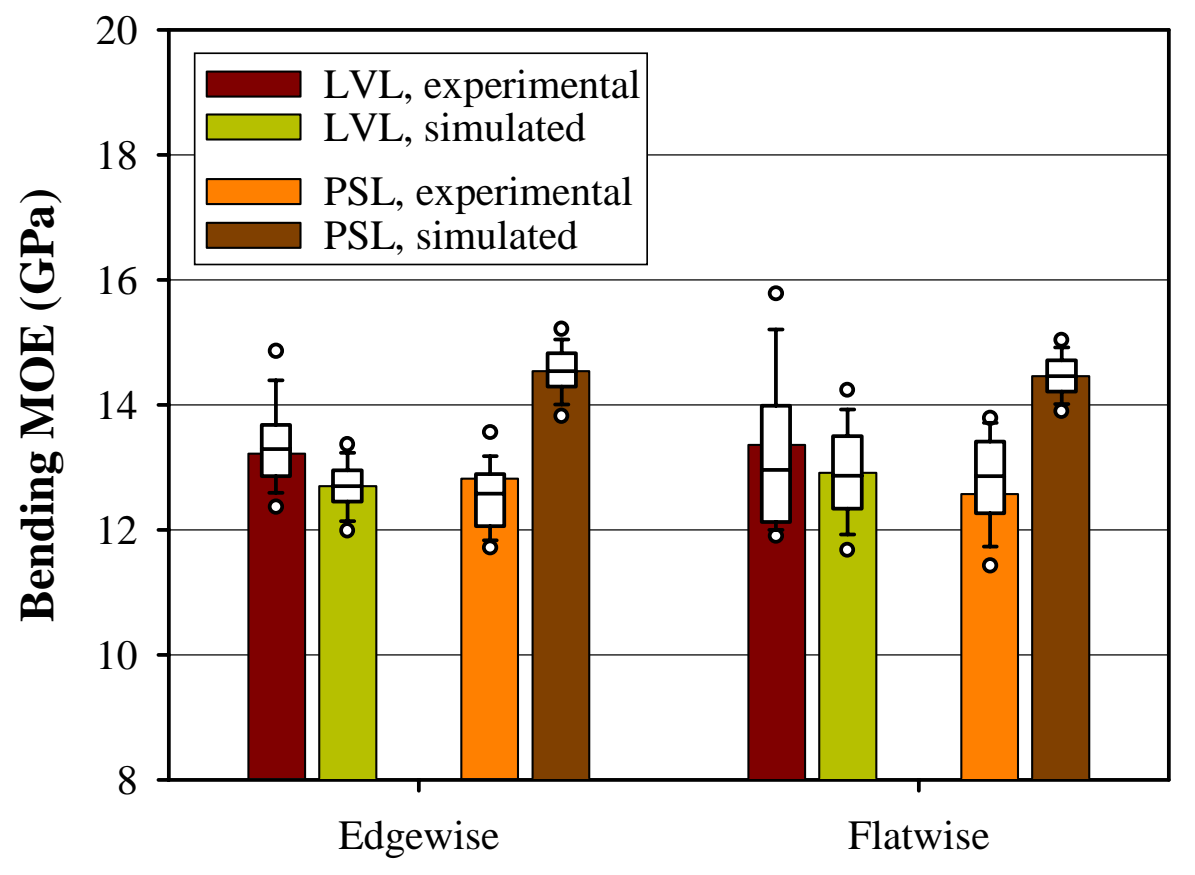

Figure 7.4 - Comparison of the experimental and simulated flatwise and edgewise bending MOE of LVL and PSL

(box plots for the simulation results are based on all 400 generated values.) 
compression MOE. The imperfect stacking of the strands might lead to weakened adhesion or glueline discontinuities, which would decrease the bending MOE, because the gluelines cannot effectively transfer loads from one strand to another. The fact that the model does not account for southern yellow pine strands in experimental beams might further bias results. Although these strands are supposed to be stronger than yellowpoplar, nothing is known about the effect of densification on the MOE of southern yellow pine veneer, which might conceivably be less pronounced than in yellow poplar. The above effects combined might account for the differences between the simulated and experimental results.

Information from the manufacturer also revealed that quality control measurements indicate significantly higher bending MOE values than the values in Table 6.11. It is possible that, for some reason, available beams were inferior to manufactured PSL material in general. In this case, simulation results might represent the composite better than the measured elastic parameters reported in section 6.3.2.

The degree of association between the simulated flatwise and edgewise MOE of LVL and PSL was assessed by calculating the regression parameters for each simulation run. The average of the $\mathrm{r}^{2}$ values for the two composites were 0.299 and 0.353 , respectively. This agrees reasonably well with the $\mathrm{r}^{2}$ value of 0.342 for LVL, shown on Figure 6.17 a. The simulated $r^{2}$ value for PSL is somewhat higher than the one experienced in reality. However, the $r^{2}$ value of PSL was very variable between the simulation runs, and in some cases (e.g. simulation nr. 4 or nr. 8 - see Table E.18) it was very close to that shown on Figure 6.17 b. 


\subsubsection{Validation of the orthotropic compression MOE simulation}

The average statistical parameters of the twenty compression MOE simulations in the six chosen directions are shown in Table 7.3. APPENDIX $\boldsymbol{F}$ contains the statistics for each simulation run individually. Figure 7.5 compares the simulation results in each direction for LVL and PSL to the experimental results in Table 6.14.

In terms of the longitudinal compression MOE - which is the most important of the six directions - the simulation performed exceptionally well for both composites. The longitudinal compression MOE of PSL is estimated with excellent accuracy, while that of LVL is only slightly underestimated. In other directions in the $x y$ plane the simulation routines usually worked with reasonable accuracy. Outside of this plane the models significantly overestimated the MOE values. The reason for this is unknown. Since these directions are rarely of interest, the inaccuracy of the simulated results in these cases is not a very serious problem.

It is important to mention that the standard deviation of the simulated MOE values was consistently smaller than that of the experimental data. One possible explanation of this inconsistency is random measurement error introduced in the compression MOE determination, which would increase the spread, but not bias the experimental values. One needs to exercise caution when interpreting the simulation results, or trying to establish allowable values from them. 
Table 7.3 - Simulated compression MOE of LVL and PSL in six directions

\begin{tabular}{llrrrrrr}
\hline \hline & \multicolumn{1}{c}{$\begin{array}{c}E_{x} \\
(\mathrm{GPa})\end{array}$} & $\begin{array}{c}E_{y} \\
(\mathrm{GPa})\end{array}$ & \multicolumn{1}{c}{$\begin{array}{c}E_{z} \\
(\mathrm{GPa})\end{array}$} & $\begin{array}{c}E_{45^{\circ}}^{0^{\circ}} \\
(\mathrm{GPa})\end{array}$ & $\begin{array}{c}E_{45^{\circ}}^{90^{\circ}} \\
(\mathrm{GPa})\end{array}$ & \multicolumn{1}{c}{$\begin{array}{c}E_{90^{\circ}}^{45^{\circ}} \\
(\mathrm{GPa})\end{array}$} \\
\hline \multirow{4}{*}{$\mathbf{L V L}$} & Mean & $\mathbf{1 1 . 3 3}$ & $\mathbf{0 . 4 1}$ & $\mathbf{0 . 8 2}$ & $\mathbf{0 . 7 9}$ & $\mathbf{1 . 5 2}$ & $\mathbf{0 . 4 9}$ \\
& $\mathrm{s}$ & 0.46 & 0.01 & 0.03 & 0.02 & 0.06 & 0.02 \\
& Min & 10.61 & 0.39 & 0.77 & 0.75 & 1.43 & 0.47 \\
& Max & 12.11 & 0.43 & 0.87 & 0.83 & 1.61 & 0.51 \\
& Skewness & 0.065 & 0.108 & 0.146 & 0.109 & 0.124 & 0.170 \\
\hline \multirow{4}{*}{ PSL } & Mean & $\mathbf{1 3 . 3 4}$ & $\mathbf{0 . 5 4}$ & $\mathbf{1 . 0 2}$ & $\mathbf{1 . 0 6}$ & $\mathbf{1 . 9 0}$ & $\mathbf{0 . 6 4}$ \\
& S & 0.43 & 0.01 & 0.04 & 0.04 & 0.06 & 0.02 \\
& Min & 12.63 & 0.52 & 0.97 & 1.00 & 1.80 & 0.61 \\
& Max & 13.99 & 0.56 & 1.08 & 1.11 & 1.99 & 0.67 \\
& Skewness & -0.139 & -0.141 & -0.053 & -0.081 & -0.035 & -0.035 \\
\hline \hline
\end{tabular}

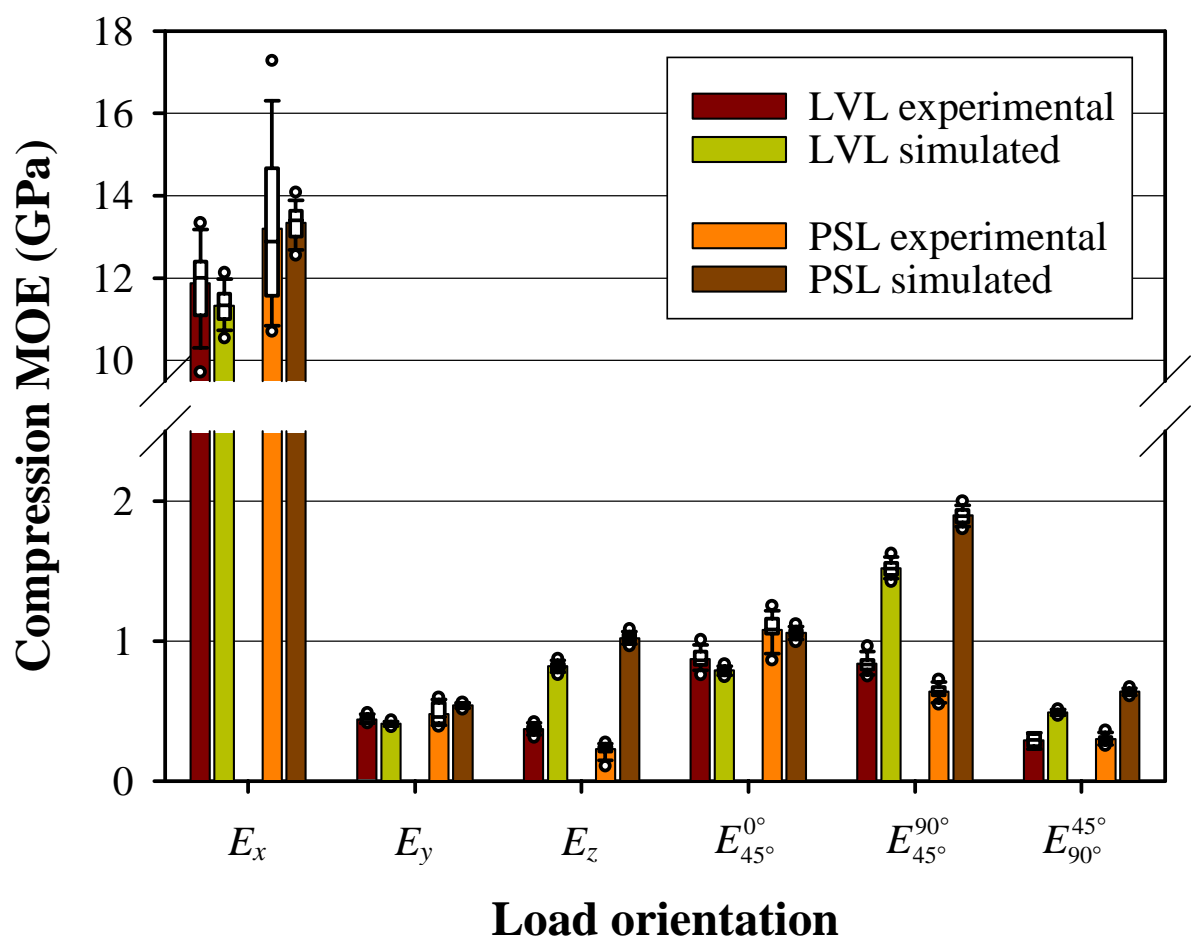

Figure 7.5 - Comparison of the experimental and simulated compression MOE of LVL and PSL in the six simulated directions (box plots for the simulation results are based on all 400 generated values.) 

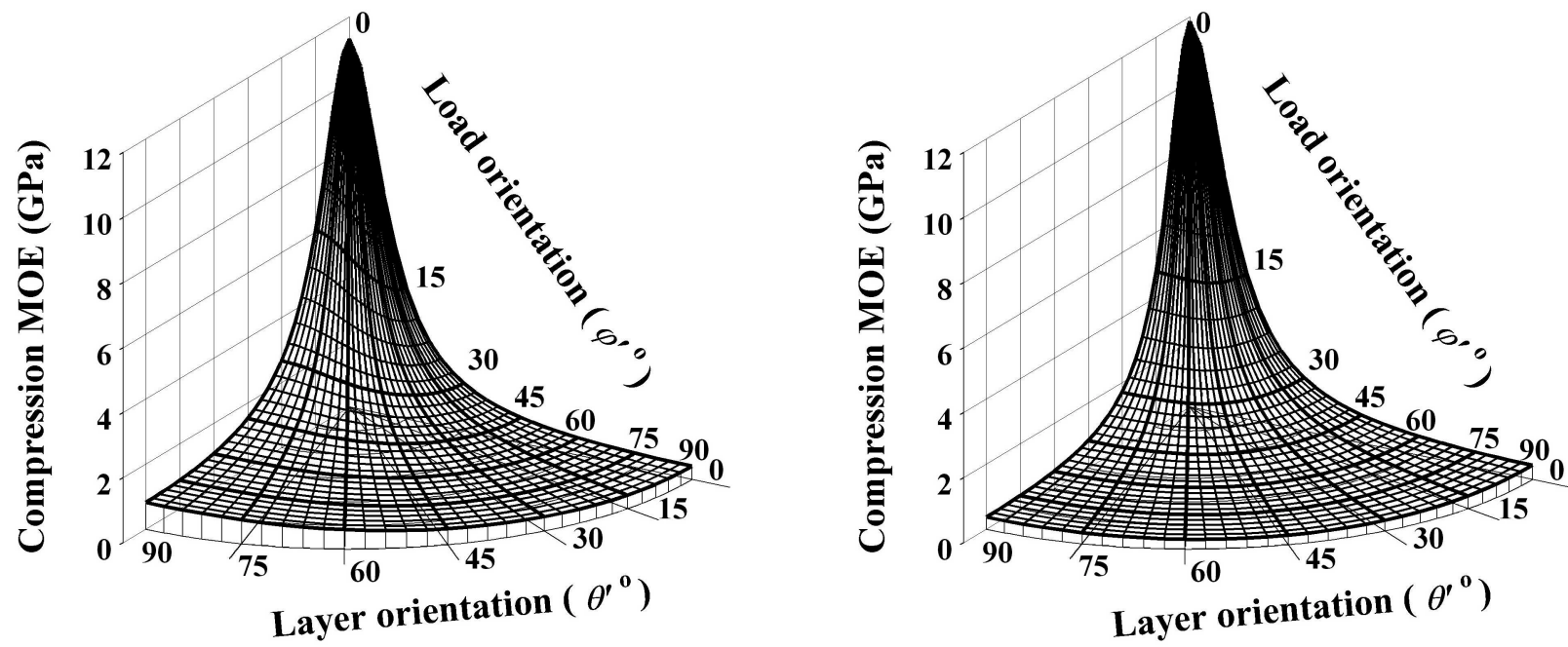

a. - LVL - simulated compression MOE

b. $-\mathrm{LVL}$ - experimental compression MOE
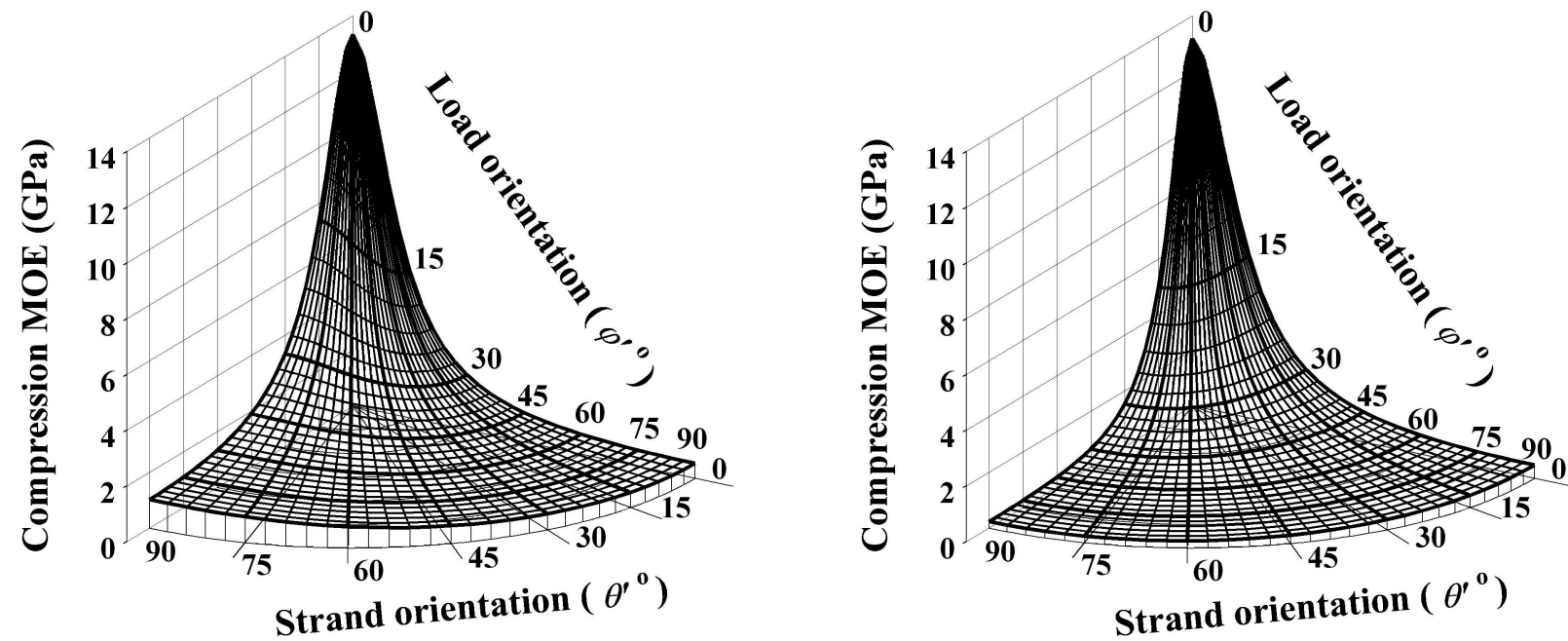

C. - PSL - simulated compression MOE

d. - PSL - experimental compression MOE

Figure 7.6 - Orthotropy diagrams created from the experimental and simulated compression MOE values of the composite materials 
Figures 7.6 a and $\mathbf{b}$ show the orthotropic compression diagrams generated from the simulated and experimental compression MOE of LVL, respectively, using the orthotropic tensor theory. Details $\mathbf{c}$ and $\mathbf{d}$ on this figure show similar diagrams for PSL. The simulated and actual compression MOE values are apparently very similar in the $x y$ plane. Although the accuracy gets worse as the layer or strand orientation $\left(\theta^{\prime}\right)$ increases, the overall efficiency of the models to predict the orthotropic compression MOE seems excellent.

\subsection{Two case studies}

To demonstrate the uses of the developed simulation models, two case studies are presented here. These procedures required minimal modification to the simulation models, consisting of simply changing some parameters. Other investigations may involve more significant programming tasks; however, using the principles described in section 7.1, the functions, and the module that contains species-specific information, described in APPENDIX $\boldsymbol{F}$, one can easily model composite materials with completely novel designs, as well.

\subsubsection{Modifying the layup of LVL}

The first case study consisted of simulating new layup combinations for LVL. Seven new setups were simulated and compared to the original, all-yellow-poplar material. Table 7.4 shows the layup combinations. The first layup is the original material 
Table 7.4 - The eight layup combinations; average edgewise and flatwise MOE

\begin{tabular}{clcc}
\hline $\begin{array}{c}\text { Comb. } \\
\text { code }\end{array}$ & Layup $^{1}$ & $\begin{array}{c}\boldsymbol{E}_{\text {edge }} \\
(\mathrm{GPa})\end{array}$ & $\begin{array}{c}\boldsymbol{E}_{\text {flat }} \\
(\mathrm{GPa})\end{array}$ \\
\hline 1 & YYYYYYYYYYYYYYY & 12.70 & 12.91 \\
2 & AAAAAAAAAAAAAA & 11.55 & 11.79 \\
3 & OOOOOOOOOOOOOOO & 12.39 & 12.59 \\
4 & OOYYYYYYYYYYYOO & 12.60 & 12.66 \\
5 & OOOOYYYYYYYOOOO & 12.54 & 12.62 \\
6 & YYYYYYAAAYYYYY & 12.49 & 12.92 \\
7 & YYYYAAAAAAYYYY & 12.18 & 12.86 \\
8 & YYYYYAAAAAOOOOO & 12.39 & 15.02 \\
\hline
\end{tabular}

$\overline{\mathrm{I}} \mathrm{A}$ - quaking aspen ; $\mathrm{O}-$ red oak ; Y - yellow-poplar. The lefthand side is the tension zone in flatwise bending.

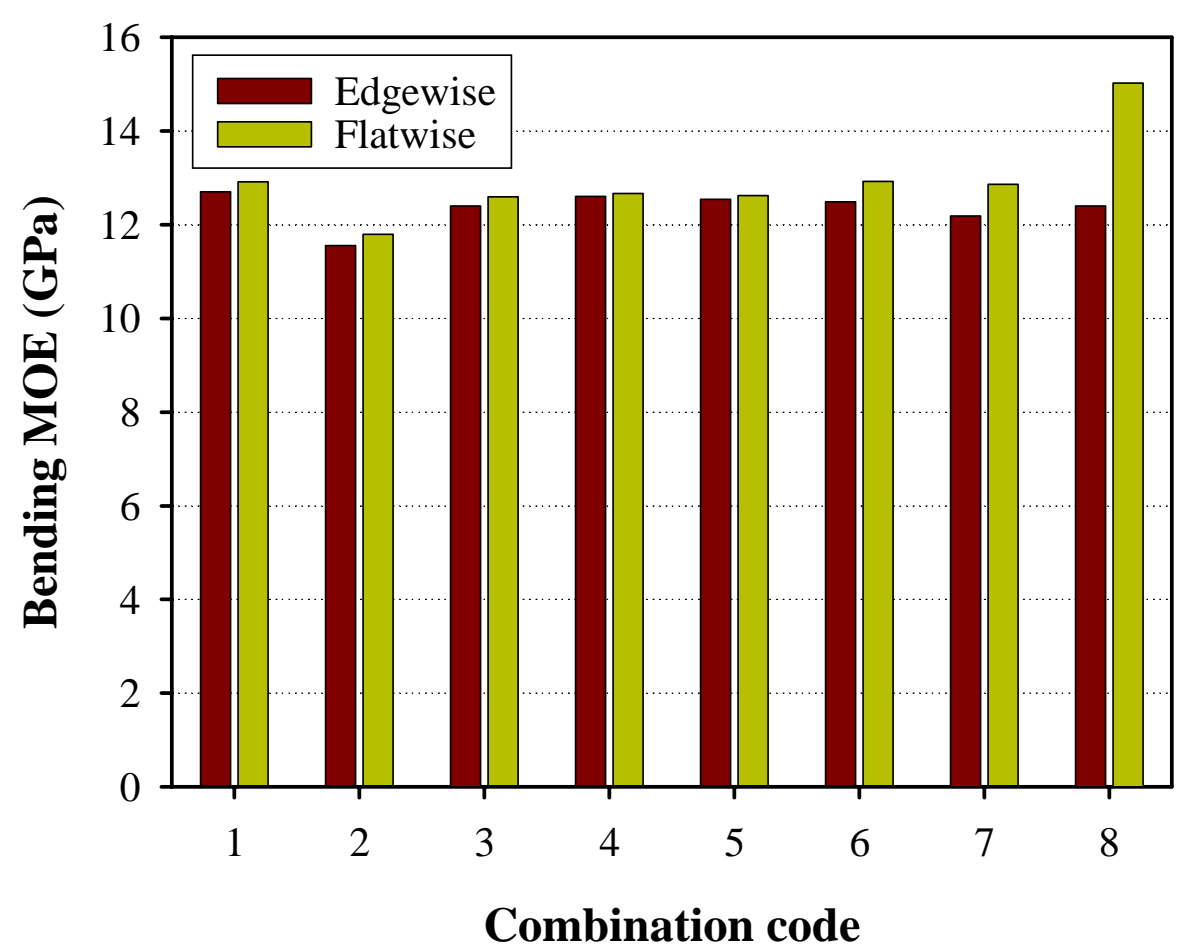

Figure 7.7 - Flatwise and edgewise bending MOE of simulated LVL beams with various layups 
used in chapter 7.2. The second and third combinations contain aspen and oak veneer only, respectively. In the fourth and fifth setup, oak veneer is substituted for the outside layers in the yellow-poplar LVL, while beams of type six and seven contain aspen material in the core. The reason to place oak on the outside and aspen in the core is to optimize the beams in flatwise bending, since oak is typically stronger, while aspen is weaker than yellow-poplar. The eighth variety is an asymmetric beam containing five layers of each species; aspen, red oak and yellow-poplar material placed in the core, in the compression zone and in the tension zone, respectively.

Table 7.4 includes the average edgewise and flatwise bending MOE simulated using the different layup combinations. Figure 7.7 demonstrates the results in a graphical form. As expected, the all-aspen LVL has somewhat lower MOE values than the original yellow poplar material. For the oak beams, the simulation model also indicated a slight drop in the elastic parameters. The same pattern was true when red oak was placed in the outside two to four layers (combinations nr. 4 and 5.) This contradicts the expectations, because oak is generally considered stronger than yellow-poplar. However, damages sustained during the peeling process significantly reduce the tensile MOE of red oak, so that it will be even lower than that of yellow-poplar. This is the reason why bending MOE does not improve when red oak is substituted for yellow-poplar in a symmetric fashion. This is an indication that introducing red oak as a raw material for LVL manufacture might not be a viable solution for the industry.

The addition of aspen material in the core of the yellow-poplar beam (combinations nr. 6 and 7) decreases the edgewise bending MOE proportionally to the volume fraction of the aspen veneers. The flatwise MOE, on the other hand, changes very 
little. If only a few layers are added to the material, neither MOE value decreases very significantly. Thus, using aspen veneer might be a feasible way to diversify the raw material base of LVL manufacture.

The edgewise MOE of layup nr. 8 is also slightly lower than that of yellow-poplar LVL. This is not surprising, since - as beams nr. 2 and 3 demonstrate - both aspen and oak tend to decrease the bending MOE of the composite. The flatwise MOE, however, shows a dramatic improvement in this case. The explanation of this effect is that oak material is located in the compression zone of the simulated beams. The compression MOE of solid oak timber, which is used in the simulation, is much better than that of the other two species. The damages that decreased the measured dynamic MOE of oak might or might not influence the compression MOE of the veneer. Caution is required, therefore, when drawing conclusions from the bending MOE simulated for beam nr. 8 .

\subsubsection{Decreasing the variation of load and strand orientation in PSL}

The purpose of the second study was to demonstrate the improvement that could be achieved in PSL if the strand angle and strand orientation would be better controlled. The investigation consisted of simulating the flatwise and edgewise MOE values of PSL, while progressively reducing the scale parameter (standard deviation) of the distribution of strand angle $(\alpha)$ and strand deviation $(\beta)$. The examined range was between $100 \%$ and $0 \%$ of the scale parameters, i.e. decreasing the variance of $\alpha$ and $\beta$ from their original level to no variation at all. The procedure assumed no change in the other parameters (e.g. strand thickness, the number of strands or the mean value of $\alpha$ and $\beta$.) 

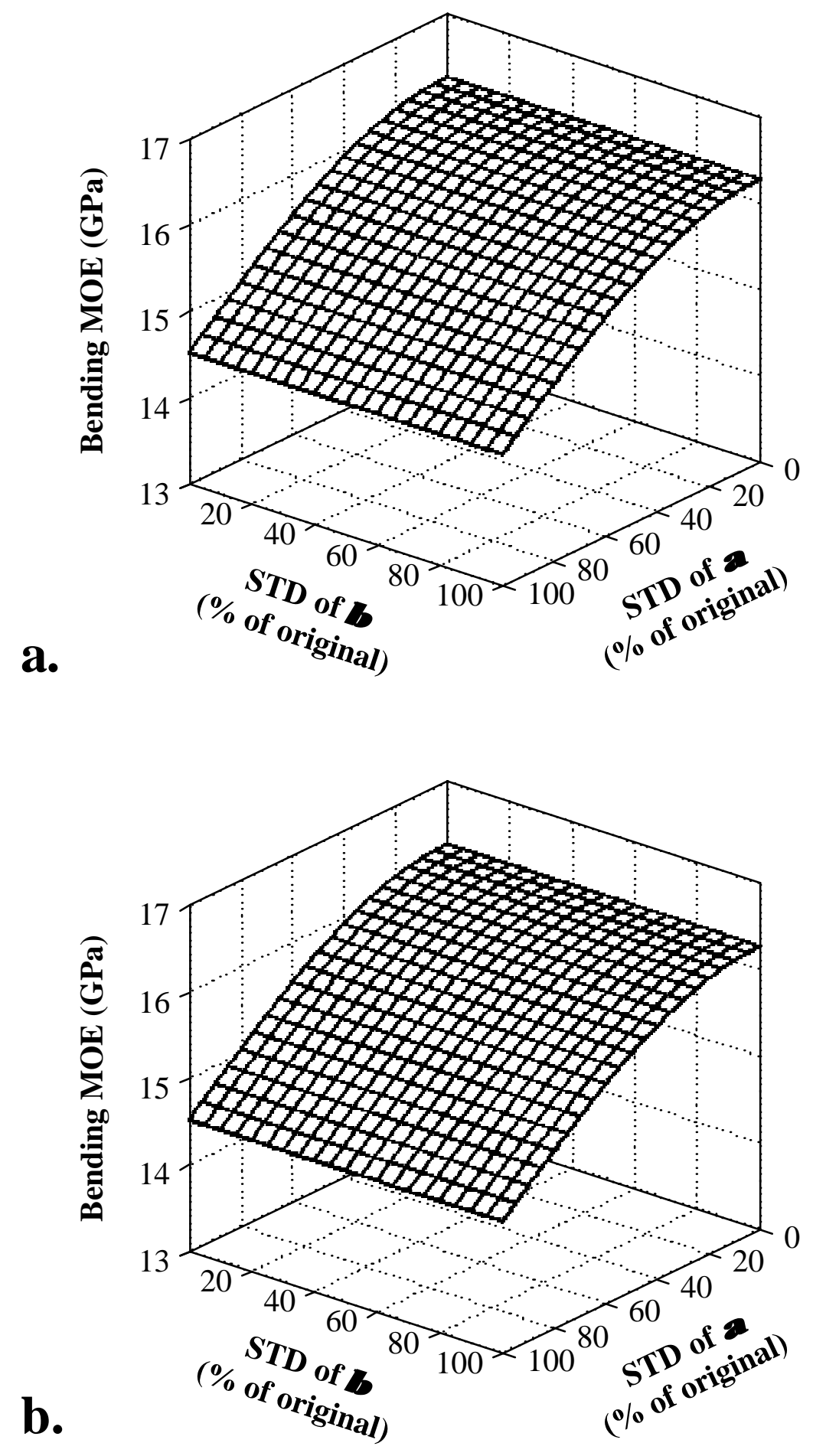

Figure 7.8 - The effect of the variance of $\alpha$ and $\beta$ on the flatwise (a) and edgewise (b) bending MOE of PSL 
Figures 7.8 a and b present the edgewise and flatwise MOE of PSL, respectively, as a function of the standard deviation of $\alpha$ and $\beta$, expressed as a percent of the original variation. As these diagrams show, decreasing the deviation of $\beta$ from its mean value has little effect on the MOE of PSL. On the other hand, changing the variance of $\alpha$ affects the elastic parameters very significantly. Bending MOE improves more than $12 \%$ (nearly 2 $\mathrm{GPa}$ ), if the variation is completely eliminated. This is unfortunately not possible for PSL. However, if, by some innovative means, the standard deviation could be reduced to $50 \%$, it would still increase MOE values by $8.5 \%$, provided that the other parameters do not change. 


\section{SUMMARY AND CONCLUSIONS}

A comprehensive investigation including the raw materials, geometric structure, mechanical properties and simulation modeling of Laminated Veneer Lumber and Parallel Strand Lumber led to the following conclusions:

- Orthotropic theories, developed through tensorial analysis, other theoretical considerations or empirical observations, estimate shear and compression strength, as well as compression and tensile elasticity of solid wood, as functions of grain and/or ring orientation, reasonably well. The orthotropic models can be used to describe the orthotropy of structural composite lumber, too, and in simulations that attempt to model the elastic properties of these materials.

- The orthotropic tensile elasticity of veneers can be successfully evaluated using the dynamic (stress-wave) MOE. An investigation, that included stress-wave timing followed by static measurements in the $0^{\circ}, 15^{\circ}, 30^{\circ}$ and $45^{\circ}$ orientations, showed that a second order correlation function represents the relationship between dynamic and static MOE in this range.

- Densification improves the MOE of hardwood veneers. The resulting functions show that MOE increases at a decreasing rate as a result of progressive densification, because of cumulative damages in the cell-wall structure. Quadratic densification functions described the relationship between density and MOE increase well. These functions can be used for simulating the effect of densification on the constituents of LVL and PSL.

- The bending and compression MOE of structural composite lumber can be modeled based on the laminate theory, and the equality of external work and internal energy, respectively. These theories require the simulation of the geometric structure and orthotropic elastic properties of the constituents in LVL and PSL. Developed models contain many deterministic and stochastic input variables, which are generated using the Monte Carlo simulation technique. 
- Simulation results usually agreed with the experimental bending and orthotropic compression MOE of LVL and PSL reasonably well. Some differences exist, however, in the bending MOE of PSL and in certain compression MOE values. Despite these discrepancies, developed models can be serviceable in estimating the effect of using different raw-materials or changing the geometric structure of the composites, as demonstrated by two case studies in chapter 7 . The principles used for the simulations and the developed database make the simulation of completely novel designs relatively simple, as well. 


\section{RECOMMENDATIONS FOR FURTHER RESEARCH}

There are several ways through which the research described in this dissertation can be continued, improved upon or extended. The predictions of the presented models can be refined by:

- Accounting for the changing cross-sections of the beams along its length. The crosssection of PSL changes in the longitudinal direction; strand positions shift according to the strand angle $(\alpha)$, strands end and new strands are introduced, etc. In LVL, new veneer sheets are introduced, and connect to old ones through overlaps, where the number of layers temporarily increases. The models do not represent any of these realities in their current form.

- The neutral axes of the beams do not exactly coincide with their symmetry axes. The determination of the actual neutral axes might improve the predictions provided by the models.

- The effect of densification on the elastic parameters of the constituents is still not very well understood. Different initial moisture contents, pressing temperatures, pressing times, and their interactions may cause variable results at similar densification levels. A more comprehensive densification study, including several combinations of the above factors, could lead to more accurate simulation results. This would also require the simulation of heat and moisture conditions within the composite during hot pressing. Such simulations are possible, as demonstrated by several works (e.g. Humphrey and Bolton 1989; Zombori 2001).

- Possible glueline imperfections and glue penetration might influence the MOE of the composites in various ways. A comprehensive study of these phenomena would greatly increase the models' accuracy. Such studies, however, are very complex and time-consuming, and results might not be straightforward to apply in a simulation study. 
- The strand-width in PSL, which is actually a random variable, is treated as a deterministic value in the simulation. Addressing this inconsistency would require little effort. However, the improvement in the simulated properties might not be very significant, since the geometric and physical parameters of the simulated beams already show good agreement with reality.

Results and principles presented in the preceding chapters might provide a basis to extend the applicability of the simulations to other raw materials, composite types or mechanical properties:

- Providing the physical and mechanical parameters, as well as assessing the effect of densification on the elastic parameters of other species that are or might be used in composite manufacture, can extend the uses of the models. If the necessary parameters are available, the model can easily incorporate further species.

- Presently, there is one more composite lumber product on the US market that the present study did not incorporate. Studying the geometric structure and parameters of Laminated Strand Lumber might provide a way to simulate the elastic properties of this composite in a similar way as those of LVL and PSL. The constituents, manufacturing process, and geometric structure of this composite, however, might require significant modifications to the main simulation routines.

- Innovative composite lumber designs might emerge, as well. Introduction of such composites requires considerable investment, which might not be realized if the composite fails to fulfill consumer requirements. Modifying the simulation routines can enable them to predict the MOE of the hypothesized composites, and forecast their mechanical performance at an early stage of development. 
- Finally, it is the hope of the author that the presented work might trigger and aid the development of further simulations that estimate other mechanical properties of wood-based composite lumber, such as bending, compression or shear strength. Unfortunately, creating such models is less straightforward than the simulation of elastic properties; however, these properties are very important in many applications, and their estimation is possible, as demonstrated by various researchers. 


\section{REFERENCES}

American Society for Testing and Materials. 1994. Standard Methods of Testing Small Clear Specimens of Timber. ASTM D 143 - 94. ASTM, West Conshohocken, Pa.

American Society for Testing and Materials. 1994. Standard Methods of Static Tests of Lumber in Structural Sizes. ASTM D 198 - 94. ASTM, West Conshohocken, Pa.

American Society for Testing and Materials. 1993. Standard Test Methods for Specific Gravity of Wood and Wood-base Materials. ASTM D 2395 - 93. ASTM, West Conshohocken, $\mathrm{Pa}$.

American Society for Testing and Materials. 1992. Standard Test Methods for Direct Moisture Content Measurement of Wood and Wood-base Materials. ASTM D 4442 92. ASTM, West Conshohocken, Pa.

Anderson, R. B. 1983. Furniture Rough Mill Costs Evaluated by Computer Simulation. Res. Pap. NE-518 USDA Forest Service, NE Forest Exp. Station, Broomall, PA. 11 pp.

Arcan, M., Z. Hashin and A. Voloshin, 1978. A Method to Produce Uniform Plane-stress States with Applications to Fiber-reinforced Materials. Experimental Mechanics 18(4): 141-146.

Armstrong, J. P., D. W. Patterson and J. E. Sneckenberger. 1991. Comparison of Three Equations for Predicting Stress Wave Velocity as a function of grain angle. Wood and Fiber Sci. 23(1):32-43.

Ashkenazi, E. K. 1959. On the Problem of Anisotropy of Construction Materials. Sov. Phys. Tech. Phys. Vol 4. 1959 pp. 333-338. (not seen)

Ashkenazi, E. K. 1978. Anisotropy of Wood and Wood-Base Materials. First edition.(in Russian) Izdatelstvo Lesnaja Promuslennosty. Moscow, USSR. (not seen)

Barnes, D. 2001. A Model of the Effect of Strand Length and Strand Thickness on the Strength Properties of Oriented Wood Composites. Forest Prod. J. 51(2):36-46.

Bendsten, B. A., and S. Porter 1978. Comparison of Results From Standard 2-Inch Width 1-1/2-Inch Shear Block Tests. Forest Prod. J. 28(7):54-56.

Bendsten, B. A., J. H. Haskell and W. L. Galligan 1978. Characteraizing the StressCompression Relationship of Wood In Compression Perpendicular to Grain. Wood Sci. 10(3):111-121.

Bindzi, I. and M. Samson. 1995. New Formula for Influence of Spiral Grain on bending stiffness of Wooden Beams. ASCE, Journal of Structural Engineering, 121(11):15411546.

Bodig, J. 1965. The Effect of Anatomy on the Initial Stress-Strain Relationship in Transverse Compression. Forest Prod. J. 15(4):197-202.

Bodig, J. and J. R. Goodman. 1972. Prediction of Elastic Parameters of Wood. Wood Sci. 5(4):249-264. 
Bodig, J. and B. A. Jayne, 1982. Mechanics of Wood and Wood Composites. Van Nostrand Reinhold Co., N.Y. 712 pp.

Bohlen, J. C. 1972. Laminated Veneer Lumber - Development, and Economics. Forest Prod. J. 22(1):18-26.

Bryant, B. S. 1966. The Chemical Modification of Wood from the Point of View of Wood Science and Economics. Forest Prod. J. 16(2):20-27.

Cowin, S. C. 1979. On the Strength Anisotropy of Bone and Wood. J. of Applied Mech. 46(12):832-838.

Dai, C. and P. R. Steiner. 1993. Compression Behavior of Randomly Formed Wood Flake Mats. Wood and Fiber Sci. 25(4):349-358.

Dai, C. and P. R. Steiner. 1994a. Spatial Structure of Wood Composites in Relation to Processing and Performance Characteristics. Part 2. Modelling and Simulation of a Randomly-Formed Flake Layer Network. Wood Sci. Technol. 28:135-146.

Dai, C. and P. R. Steiner. 1994b. Spatial Structure of Wood Composites in Relation to Processing and Performance Characteristics. Part 3. Modelling the Formation of Multi-Layered Random Flake Mats. Wood Sci. Technol. 28:229-329.

Divos, F., I. Daniel and L. Bejo. 2000. Defect Detection in Timber by Stress Wave Technique. In: Proc. Int. Conf. on Wood and Wood Fiber Composites, Stuttgart, Germany, 13-15 April, 2000.

Divos, F., T. Tanaka. 2000. Effect of creep on Modulus of Elasticity determination of wood. ASME J. of Vibration and Acoustics 122(1):90-92.

Divos, F. 2000. Ultrasonic Timer - User's Guide. Private ed. Sopron, Hungary. 8 pp.

Dowdy, S. and S. Wearden. 1991. Statistics for Research. $2^{\text {nd }}$ ed. John Wiley and Sons, New York, NY. 259 pp.

Ethington, R. L., V. Eskelsen and R. Gupta 1996. Relationship Between Compression Strength Perpendicular to Grain and Ring Orientation. Forest Prod. J. 46(1):84-86

Forest Products Laboratory. 1999. Wood Handbook: Wood as an Engineering Material. Washington DC: U.S. Department of Agriculture.

FPL-Press-Lam Research Team. 1972. Feasibility of Producing a High-Yield Laminated Structural Product. Res. Pap. FPL 175. USDA Forest Service, Forest Products Laboratory, Madison, WI.

Geimer, R. L., R. J. Mahoney, S. P. Loehnertz and R. W. Meyer. 1985. Influence of Processing-Induced Damage on Strength of Flakes and Flakeboards. Res. Pap. FPL 463. USDA Forest Service, Forest Products Laboratory, Madison, WI.

Gerhards, G. C. 1988. Effect of Slope of Grain on Tensile Strength. Forest Prod. J. 38(7/8):39-40.

Goodman, J. R., and J. Bodig 1972. Orthotropic Strength of Wood in Compression. Wood Sci. 4(2):83-94 
Hall, P. 1988. Introduction to the Theory of Coverage Processes. John Wiley and Sons, New York.

Hankinson, R. L. 1921. Investigation of Crushing Strength of Spruce ate Varying Angles of Grain. Air Service Information Circular No. 259, U.S. Air Service, 1921. (not seen)

Harless, T. E., F. G. Wagner, R. D. Seale, P. H. Mitchell and D. S. Ladd. 1987. A Model to Predict the Density Profile of Particleboard. Wood Fiber Sci. 19(1):81-92.

Harris, R. A. and J. A. Johnson. 1982. Characterization of Flake Orientation in Flakeboard by the Von Mises Probability Distribution Function. Wood and Fiber 14(4):254-266.

Humphrey, P. E. and A. J. Bolton. 1989. The Hot Pressing of Dry-formed Wood-based Composites. Part II. A Simulation Model for Heat and Moisture Transfer, and Typical Results. Holzforschung 43:199-206.

Hunt, M. O., M. H. Triche, G. P. McCabe and W. L. Hoover. 1989. Tensile Properties of Yellow-Poplar Veneer Strands. Forest Prod. J. 39(9):31-33.

Jung, J. 1979. Stress-Wave Grading Techniques on Veneer Sheets. Gen. Tech Rep. FPLGTR-27. USDA Forest Service, Forest Products Laboratory, Madison, WI.

Jung, J. 1982. Properties of Parallel-Laminated Veneer from Stress-Wave-Tested Veneers. Forest Prod. J. 32(7):30-35.

Jung, J. 1984. Investigation of Various End Joints in Parallel-Laminated Veneer. Forest Prod. J. 34(5):51-55.

Kallmes, O. and H. Corte. 1960. The Structure of Paper. I. The Statistical Geometry of an Ideal Two Dimensional Fiber Network. TAPPI 43(9):737-752.

Kallmes, O., H. Corte. and G. Bernier. 1961. The Structure of Paper. II. The Statistical Geometry of a Multiplanar Fiber Network. TAPPI 44(7):519-528.

Kaiserlik, J. H. and R. F. Pellerin. 1977. Stress-Wave Attenuation as an Indicator of Lumber Strength. Forest Prod. J. 27(6):39-43.

Kim, K. Y. 1986. A note on Hankinson formula. Wood and Fiber Sci., 18(2):345-348.

Kimmell, J. D. and J. J. Janiowak. 1995. Red Maple and Yellow-Poplar LVL from Ultrasonically Rated Veneer. Forest Prod. J. 45(7/8):54-58.

Koch, P. 1967. Location of Laminae by Elastic Modulus May Permit Manufacture of Very Strong Beams from Rotary-Cut Southern Pine Veneer. Res. Pap. SO-30 USDA Forest Service, So. Forest Exp. Station, New-Orleans, La.

Koch, P. and G. E. Woodson. 1968. Laminating Butt-Jointed, Log-Run Southern Pine Veneers into Long Beams of Uniform High Strength. Forest Prod. J. 18(10):45-51.

Kollman, F. P. P. 1934. Untersuchungen an Kiefern und Fichtenholz aus der Rheinpfalz. Forstwiss. Cbl. 56(6):181-189. (not seen)

Kollman, F. P. P. and W. A. Cote Jr. 1968. Principles of Wood Science and Technology. Volume I. - Solid Wood. Springer-Verlag, New York. 592 pp. 
Kunesh, R. H. 1968. Strength an Elastic Properties of Wood In Transverse Compression. Forest Prod. J. 18(1):65-72.

Kunesh, R. H. 1978. MICRO = LAM: Structural Laminated Veneer Lumber. Forest Prod. J. 28(7):41-44.

Kurse, K., H. Thomen, H. Maurer, A Steffen and R. Leon-Mendez. 1997. Optimization of Particleboard Production by Means of Process Modeling. Holz als Roh- und Werkstoff 55:17-24.

Lang, E. M. 1997. An Alternative Method For Shear Strength Assessment. Forest Prod. J. 47(11/12):81-84.

Lang, E. M. and M. P.Wolcott. 1996a. Model for Viscoelastic Consolidation of WoodStrand Mats. Part I. Structural Characterization of the Mat vis Monte Carlo Simulation. Wood and Fiber Sci. 28(1) 1996, pp 100-109.

Lang, E. M. and M. P.Wolcott. 1996b. Model for Viscoelastic Consolidation of WoodStrand Mats. Part II. Static Stress-Strain Behavior of the Mat. Wood and Fiber Sci. 28(3) 1996, pp 369-379.

Lang, E.M. and Zs. Kovacs. 2001. Size Effect on Shear Strength Measured by the ASTM method. Forest Prod. J. 51(3):49-52.

Langwig, J. E., J. A. Mayer and R. W. Davidson. 1968. Influence of Polymer Impregnation on Mechanical Properties of Basswood. Forest Prod. J. 18(7):33-36.

Laufenberg, T. L. 1983. Parallel Laminated Veneer: Processing and Performance Research Review. Forest Prod. J. 33(9):21-28.

Law, A. M. and W. D. Kelton. 1991. Simulation Modeling and Analysis. $2^{\text {nd }}$ ed. McGraw-Hill, Inc. New York. 759 pp.

Law, A. M. and S. Vincent. 1999. User's Guide to ExpertFit ${ }^{\text {TM }}$. Averill M. Law and Associates. $74 \mathrm{pp}$.

Lenth, C. A. and F. A. Kamke. 1996a. Investigations of Flakeboard Mat Consolidation. Part I. Characterizing The Cellular Structure. Wood and Fiber Sci. 28(2):153-167.

Lenth, C. A. and F. A. Kamke. 1996b. Investigations of Flakeboard Mat Consolidation. Part II. Modeling Mat Consolidation Using Theories of Cellular Materials. Wood and Fiber Sci. 28(3):309-319.

Liu, J. Y. 1984. New Shear Strength Test for Solid Wood. Wood and Fiber Sci. 16(4):567-574.

Liu, J. Y. and Floeter, L. H. 1984. Shear Strength in Principal Plane of Wood. Journal of Engineering Mechanics, 110(6):930-936.

Lu, C., P. R. Steiner and F. Lam. 1998. Simulation Study of Wood-Flake Composite Mat Structures. Forest Prod. J. 48(5):89-93.

Luxford, R. F. 1944. Strength of Glued-Laminated Sitka Spruce Made of Rotary Cut Veneers. Army-Navy Committee: Aircraft Design Criteria No. 1512. 
Norris, C. B. 1950. Strength of Orthotropic Materials Subjected to Combined Stresses. Forest Products Laboratory, Report No. 1816. USDA Forest Service. 49 pp.

Norris, C. B. 1957. Comparison of Standard Block-Shear Test with the Panel-Shear Test. Forest Prod. J. 7(9)299-301.

Okkonen, E. A. and B. H. River 1989. Factors Affecting the Strength of Block-shear Specimens. Forest Prod. J. 39(1):43-50.

Palardy, R. D, B. A. Haataja, S. M. Shaler, A. D. Williams and T. L. Laufenberg. 1989. Pressing of Wood Composite Panels at Moderate Temperature and High Moisture Content. Forest Prod. J. 39(4):27-32.

Pellerin, R. F. 1965. Vibrational Approach to Nondestructive Testing of Structural Lumber. Forest Prod. J. 15(3):93-101.

Price, E. W. 1976. Determining Tensile Properties of Sweetgum Veneer. Forest Prod. J. 26(10):50-53.

$\mathrm{Pu}, \mathrm{J}$. and R. C. Tang. 1997. Nondestructive Evaluation of Modulus of Elasticity of Southern Pine LVL: Effect of Veneer Grade and Relative Humidity. Wood and Fiber Sci. 29(3):249-263.

Pugel, A. D. 1990. Angle-to-Grain Tensile Strength Specimen for Thin Wood Samples. Forest Prod. J. 40(2):49-51.

Radcliffe, B.M. 1965. A theoretical evaluation of Hankinson formula for modulus of elasticity of wood at an angle to the grain. Quarterly Bull. Michigan Agr. Exp. Station. 48(2):286-295.

Rammer, D. R. and J. J. Zahn. 1997. Determination of Ylinen's Parameter for ParallelStrand Lumber. Journal of Structural Engineering 123(10):1409-1414.

Riccati, J. 1747. Verae et Germanae virim elasticarum leges ex phaenumenis demonstratae. De Bononiensi Schientarium Academia Commentarii 1:523. Bogona. (not seen)

Rippy, R. C., F. G. Wagner, T. M. Gorman, H. D. Layton and T. Bodenheimer. 2000. Stress-Wave Analysis of Douglas-Fir Logs for Veneer Properties. Forest Prod. J. 50(4):49-52.

Riyanto, D. S., and R. Gupta 1996. Effect of Ring Angle on Shear Strength Parallel to the Grain of Wood. Forest Prod. J. 46(7/8):87-92.

Ross, R. J., S. W. Willits, W. von Segen, T. Black, B. K. Brashaw and R. F. Pellerin. 1999. A Stress Wave Based Approach to NDE of Logs for Assessing Potential Veneer Quality. Part 1. Small Diameter Ponderosa Pine. Forest Prod. J. 49(11/12):60-62.

Sharp, D. J., 1985. Non-Destructive Techniques for Manufacturing LVL and Predicting Performance. Proc. Fifth Symp. on Nondestructive Testing of Wood, Washington State Univ., Pullman, Wash. pp. 100-108. 
Shuppe, T. F., C. Y. Hse, L. H. Groom and E. T. Choong. 1997. Effect of Silvicultural Practice and Veneer Grade Layup on Some Mechanical Properties of Loblolly Pine LVL. Forest Prod. J. 47(9):63-69.

Siau, J. F. 1995. Wood: Influence of Moisture on Physical Properties. Dept. of Wood Science and Forest Products, Virginia Polytechnic Inst. and State University. 227 pp.

Siau, J. F., R. W. Davidson, J. A. Mayer and C. Skaar. 1968. A Geometric Model for Wood-Polymer Composites. Wood Sci. 1(2):116-128.

Song, D. and S. Ellis. 1997. Localized Properties in Flakeboard: A Simulation Using Stacked Flakes. Wood and Fiber Sci. 29(4):353-363.

SPSS Inc. 1997. SigmaPlot® 4.0 for Windows. SPSS Inc. Chicago, IL.

Steiner, P. R. and C. Dai. 1993. Spatial Structure of Wood Composites in Relation to Processing and Performance Characteristics. Part 1. Rationale for Model Development. Wood Sci. Technol. 28:45-51.

Suchsland, O. 1967. Behavior of a Particleboard Mat during the Press Cycle. Forest Prod. J. 17(2):51-57.

Suchsland, O. and J. D. McNatt. 1986. Computer Simulation of Laminated Wood Panel Warping. Forest Prod. J. 36(11/12):16-23.

Suchsland, O. and H. Xu. 1989. A Simulation of the Horizontal Density Distribution in a Flakeboard. Forest Prod. J. 39(5):29-33.

Suchsland O. and H. Xu. 1991. Model Analysis of Flakeboard Variables. Forest Prod. J. 41(11/12):55-60.

Suhling, J. C., R. E. Rowlands, M. W. Johnson and D. E. Gunderson. 1985. Tensorial Strength Analysis of Paperboard. Experimental Mechanics. Vol. 42, 75-84.

Szalai, J. 1994. Anisotropic Strength and Elasticity of Wood and Wood Based Composites. Private ed. Sopron, Hungary. 398 pp. (in Hungarian.)

Taneda, K., I. Hasegawa and H. Kawakami. 1971. Dependence of the Rule of Mixture about Mechanical Properties of Wood-Plastic Composites. J. Japan Wood Res. Soc. 17(6):181-187. (Mokuzai Gakkaishi)

Triche, M. H. and M. O. Hunt. 1993. Modeling of Parallel-aligned Wood Strand Composites. Forest Prod. J. 43(11/12):33-44.

Tsai, W. S. and E. M. Wu. 1971. A General Theory of Strength for Anisotropic Materials. Journal of Composite Materials. 5(1):58-80.

Vlosky, R. P., P. M. Smith, P. R. Blankenhorn and M. P. Haas. 1994. Laminated Veneer Lumber: A United States Market Overview. Wood and Fiber Sci. 26(4):456-466.

Wang, K. and F. Lam. 1998. Robot-based Research on Three-layer Oriented Flakeboards. Wood and Fiber Sci. 1998 pp. 339-347.

Wang, J., J. M. Biernacki and F. Lam. 2001. Nondestructive Evaluation of Veneer Quality Using Acoustic Wave Measurements. Wood Sci. Technol. 34(2001) 505-516. 
Woodward, C. B. and J. Minor. 1988. Failure Theories for Douglas-Fir in Tension. Journal of Structural Engineering 114(12):2808-2813.

Wu, E. M. 1974. Phenomenological Anisotropic Failure Criterion. In Composite Materials, Vol. II entitled: Mechanics of Composite Materials. Sendeckyj, G. P. ed., Academic Press.

$\mathrm{Xu}, \mathrm{W} .1999$. Influence of Vertical Density Distribution on Bending Modulus of Elasticity of Wood Composite Panels: A Theoretical Consideration. Wood and Fiber Sci. 31(3):277-282.

$\mathrm{Xu}, \mathrm{W} .2000$. Influence of Percent Alignment and Shelling Ratio on Modulus of Elasticity of Oriented Strandboard: A Model Investigation. Forest Prod. J. 50(10):43-47.

$\mathrm{Xu}, \mathrm{W}$. and P. R. Steiner. 1995. A Statistical Characterization of the Horizontal Density Distribution in Flakeboard. Wood Fiber Sci. 27(2):160-167.

Xu, W. and O. Suchsland. 1997. Linear Expansion of Wood Composites: a Model. Wood Fiber Sci. 29(3):272-281.

$\mathrm{Xu}, \mathrm{W}$. and O. Suchsland. 1998a. Influence of Out-of-Plane Orientation of Particles on Linear Expansion of Particleboard: A Simulation Study. Forest Prod. J. 48(6):85-87.

Xu, W. and O. Suchsland. 1998b. Modulus of Elasticity of Wood Composite Panels with Uniform Vertical Density Profile: A Model. Wood and Fiber Sci. 30(3):293-300.

Xu, W. and P. M. Winistorfer. 1996. Fitting an Equation to Density Profile Data Using Fourier Analysis. Holz Roh- Werkst. 54(1):57-59.

Ylinen, A. 1963. A Comparative Study of Different Types of Shear Tests of Wood. Paper presented on the Fifth Conference of Wood Technology. U.S. Forest Products Laboratory, Madison, Wisconsin, September 16-27, 67 pp.

Zombori, B. G. 2001. Modeling the Transient Effects During the Hot-Pressing of WoodBased Composites. Doctoral Dissertation, Virginia Polytechnic Inst. and State University 


\section{APPENDIX A - DERIVATION OF THE ORTHOTROPIC TENSOR MODEL}

Using the elastic parameters of wood, the compliance matrix of an orthotropic material can be written in the following form:

$$
\left[\begin{array}{cccccc}
\frac{1}{E_{L}} & -\frac{v_{R L}}{E_{R}} & -\frac{v_{T L}}{E_{T}} & 0 & 0 & 0 \\
-\frac{v_{L R}}{E_{T}} & \frac{1}{E_{R}} & -\frac{v_{T R}}{E_{T}} & 0 & 0 & 0 \\
-\frac{v_{L T}}{E_{L}} & -\frac{v_{R T}}{E_{R}} & \frac{1}{E_{T}} & 0 & 0 & 0 \\
0 & 0 & 0 & \frac{1}{G_{R T}} & 0 & 0 \\
0 & 0 & 0 & 0 & \frac{1}{G_{T L}} & 0 \\
0 & 0 & 0 & 0 & 0 & \frac{1}{G_{L R}}
\end{array}\right]
$$

where: $E_{i}$ - modulus of elasticity of wood in the $i$ anatomical direction;

$G_{i j}$ - shear modulus in the main anatomical planes, where $i$ is the direction normal to the sheared plane and $j$ is the direction of the applied load;

$v_{i j}$ - Poisson ratio ( $i$ is the direction of passive strain and $j$ is the direction of applied load.

Using the relationships between the elastic parameters of wood, it can be shown that

$$
-\frac{v_{i j}}{E_{j}}=\frac{1}{2}\left(\frac{4}{E_{i j}^{45^{\circ}}}-\frac{1}{E_{i}}-\frac{1}{E_{j}}-\frac{1}{G_{i j}}\right)
$$

where: $E_{i j}^{45^{\circ}}-$ The modulus of elasticity of wood in the $i j$ plane, at a $45^{\circ}$ direction between $i$ and $j$. 
Using the above relationship, the elements of the compliance matrix can be defined in a way that makes their experimental determination easier:

$$
\left[\begin{array}{cccccc}
\frac{1}{E_{L}} & \frac{1}{2}\left(\frac{1}{E_{45^{\circ}}^{0^{\circ}}}-\frac{1}{E_{L}}-\frac{1}{E_{R}}-\frac{1}{G_{R L}}\right) & \frac{1}{2}\left(\frac{1}{E_{45^{\circ}}^{90}}-\frac{1}{E_{L}}-\frac{1}{E_{T}}-\frac{1}{G_{T L}}\right) & 0 & 0 & 0 \\
\frac{1}{2}\left(\frac{1}{E_{45^{\circ}}^{0^{\circ}}}-\frac{1}{E_{L}}-\frac{1}{E_{R}}-\frac{1}{G_{R L}}\right) & \frac{1}{E_{R}} & \frac{1}{2}\left(\frac{1}{E_{90^{\circ}}^{45^{\circ}}}-\frac{1}{E_{R}}-\frac{1}{E_{T}}-\frac{1}{G_{R T}}\right) & 0 & 0 & 0 \\
\frac{1}{2}\left(\frac{1}{E_{45^{\circ}}^{90}}-\frac{1}{E_{L}}-\frac{1}{E_{T}}-\frac{1}{G_{T L}}\right) & \frac{1}{2}\left(\frac{1}{E_{90^{\circ}}^{45^{\circ}}}-\frac{1}{E_{R}}-\frac{1}{E_{T}}-\frac{1}{G_{R T}}\right) & \frac{1}{E_{T}} & 0 & 0 & 0 \\
0 & 0 & 0 & \frac{1}{G_{R T}} & 0 & 0 \\
0 & 0 & 0 & 0 & \frac{1}{G_{T L}} & 0 \\
0 & 0 & 0 & 0 & 0 & \frac{1}{G_{R L}}
\end{array}\right]
$$

The above matrix is a simplified two-dimensional representation of a fourdimensional tensor. According to the transformation rules of a four-dimensional tensor, when the coordinate system is rotated around its origin, an element of the new tensor can be calculated using the above formula:

$$
t_{i^{\prime} j^{\prime} k^{\prime} l^{\prime}}=\Sigma t_{i j k l} \beta_{i^{\prime}}^{i} \beta_{j^{\prime}}^{j} \beta_{k^{\prime}}^{k} \beta_{l^{\prime}}^{l}
$$

where: $t_{i^{\prime} j^{\prime} k^{\prime} l^{\prime}} \quad-$ an element of the new tensor $\left(i^{\prime}, j^{\prime}, k^{\prime}, l^{\prime}=x, y, z\right)$

$t_{i j k l} \quad-$ an element of the original tensor $(i, j, k, l=L, R, T)$

$\beta_{i^{\prime}}^{i} \quad$ - the length of the orthogonal projection of a unit vector in the direction of the $i^{\text {th }}$ coordinate axis in the new coordinate system, on the $i^{\text {th }}$ axis in the old coordinate system (Figure A.1 shows three examples, where $i^{\prime}=x$ and $i=L, R, T$.)

Let the new coordinate system be chosen so that its $x$ axis is aligned with the compression load, using the definition of $\varphi$ and $\theta$, given on Figure 4.5. In this case, transforming the first element of the tensor $\left(t_{L L L L}=1 / E_{L}\right)$, will provide the reciprocal of the 


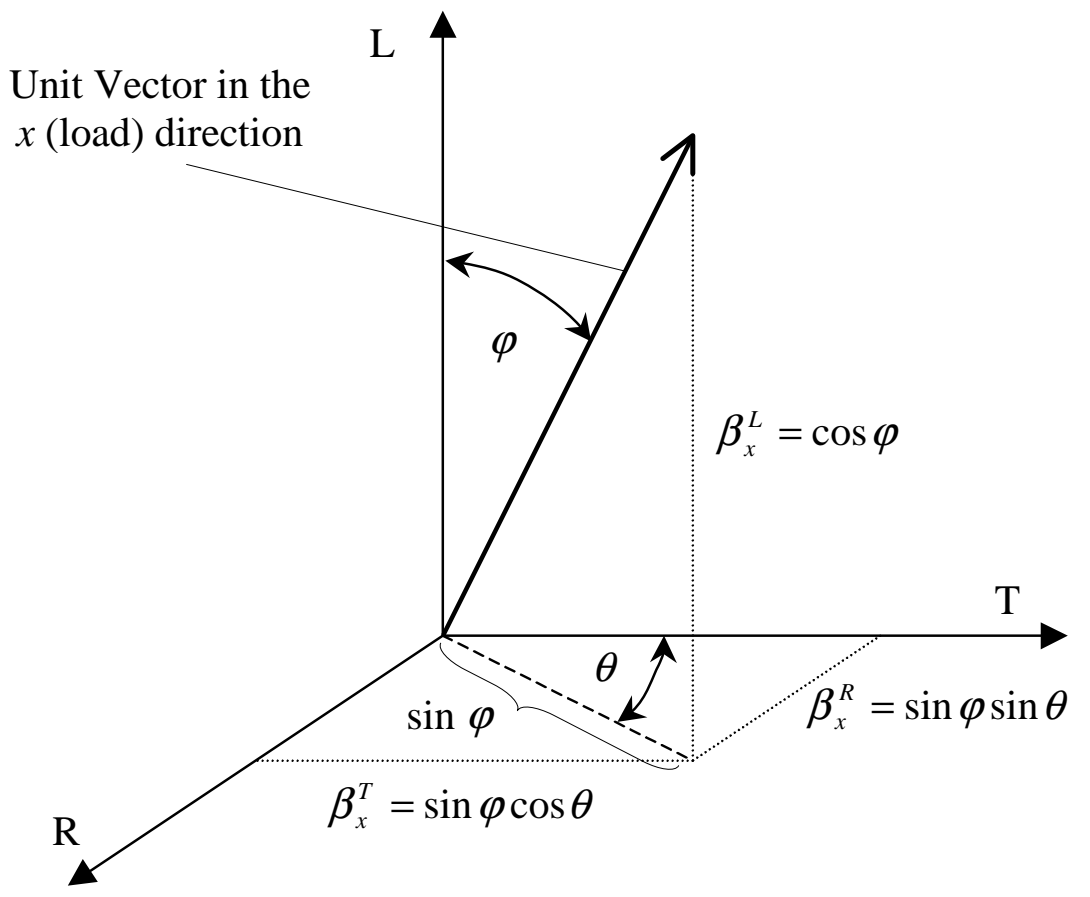

Figure A.1 - The definition of $\beta_{x}^{L}, \beta_{x}^{R}$, and $\beta_{x}^{T}$.

MOE in the $x$ direction, i.e. the direction designated by $\varphi$ and $\theta\left(t_{x x x x}=1 / E_{x}=1 / E_{\varphi}^{\theta}\right)$. The transformation requires the determination of $\beta_{x}^{L}, \beta_{x}^{R}$, and $\beta_{x}^{T}$ only. Figure A.1 shows the calculation of these coordinates. Applying the transformation rule (Equation A.4) to the first element of the matrix provides the following formula for the calculation of the MOE in a chosen direction: 


$$
\begin{aligned}
\frac{1}{\hat{E}_{\varphi}^{\theta}}= & \frac{1}{E_{L}} \cos ^{4} \varphi+\frac{1}{E_{R}} \sin ^{4} \varphi \sin ^{4} \theta+\frac{1}{E_{T}} \sin ^{4} \varphi \cos ^{4} \theta \\
& +\left(\frac{4}{E_{90^{\circ}}^{40^{\circ}}}-\frac{1}{E_{R}}-\frac{1}{E_{T}}\right) \sin ^{4} \varphi \sin ^{2} \theta \cos ^{2} \theta \\
& +\left(\frac{4}{E_{45^{\circ}}^{0^{\circ}}}-\frac{1}{E_{L}}-\frac{1}{E_{T}}\right) \cos ^{2} \varphi \sin ^{2} \varphi \cos ^{2} \theta \\
& +\left(\frac{4}{E_{45^{\circ}}^{90}}-\frac{1}{E_{L}}-\frac{1}{E_{R}}\right) \cos ^{2} \varphi \sin ^{2} \varphi \sin ^{2} \theta
\end{aligned}
$$

The derivation of the tensorial formula for compression strength is based on a similar transformation of the four-dimensional strength tensor. Ashkenazi (1978) defined the elements of the fourth order strength tensor according to the matrix below:

$$
\left[\begin{array}{cccccc}
\frac{1}{\sigma_{L}} & \frac{1}{2}\left(\frac{1}{\sigma_{45^{\circ}}^{0 \circ}}-\frac{1}{\sigma_{L}}-\frac{1}{\sigma_{R}}-\frac{1}{\tau_{R L}}\right) & \frac{1}{2}\left(\frac{1}{\sigma_{45^{\circ}}^{90}}-\frac{1}{\sigma_{L}}-\frac{1}{\sigma_{T}}-\frac{1}{\tau_{T L}}\right) & 0 & 0 & 0 \\
\frac{1}{2}\left(\frac{1}{\sigma_{45^{\circ}}^{0}}-\frac{1}{\sigma_{L}}-\frac{1}{\sigma_{R}}-\frac{1}{\tau_{R L}}\right) & \frac{1}{\sigma_{R}} & \frac{1}{2}\left(\frac{1}{\sigma_{90^{\circ}}^{45^{\circ}}}-\frac{1}{\sigma_{R}}-\frac{1}{\sigma_{T}}-\frac{1}{\tau_{R T}}\right) & 0 & 0 & 0 \\
\frac{1}{2}\left(\frac{1}{\sigma_{45^{\circ}}^{90^{\circ}}}-\frac{1}{\sigma_{L}}-\frac{1}{\sigma_{T}}-\frac{1}{\tau_{T L}}\right) & \frac{1}{2}\left(\frac{1}{\sigma_{90^{\circ}}^{45^{\circ}}}-\frac{1}{\sigma_{R}}-\frac{1}{\sigma_{T}}-\frac{1}{\tau_{R T}}\right) & \frac{1}{\sigma_{T}} & 0 & 0 & 0 \\
0 & 0 & 0 & \frac{1}{\tau_{R T}} & 0 & 0 \\
0 & 0 & 0 & 0 & \frac{1}{\tau_{T L}} & 0 \\
0 & 0 & 0 & 0 & 0 & \frac{1}{\tau_{R L}}
\end{array}\right]
$$

Transforming the first element of the above matrix the same way as that of the compliance matrix, provides equation 4.10. 


\section{APPENDIX B - DETAILED RESULTS OF THE STATISTICAL PROCEDURES}

Table B.1 - ANOVA tables for the orthotropic shear strength of Appalachian hardwoods

\begin{tabular}{|c|c|c|c|c|}
\hline \multicolumn{5}{|c|}{ ASPEN } \\
\hline Source & DF & Sum of Squares & Mean Square & P value \\
\hline Angle variation & 48 & 1727 & 36.0 & $<0.001$ \\
\hline Grain angle & 6 & 1542 & 257.1 & $<0.001$ \\
\hline Ring angle & 6 & 59 & 9.8 & $<0.001$ \\
\hline Grain x Ring & 36 & 125 & 3.5 & $<0.001$ \\
\hline Random error & 364 & 111 & 0.3 & \\
\hline Total & 412 & 1838 & & \\
\hline \multicolumn{5}{|c|}{ RED OAK } \\
\hline Source & DF & Sum of Squares & Mean Square & $P$ value \\
\hline Angle variation & 48 & 3162 & 65.9 & $<0.001$ \\
\hline Grain angle & 6 & 2969 & 494.8 & $<0.001$ \\
\hline Ring angle & 6 & 51 & 8.5 & $<0.001$ \\
\hline Grain x Ring & 36 & 143 & 4.0 & $<0.001$ \\
\hline Random error & 379 & 209 & 0.6 & \\
\hline Total & 427 & 3371 & & \\
\hline \multicolumn{5}{|c|}{ YELLOW-POPLAR } \\
\hline Source & DF & Sum of Squares & Mean Square & $P$ value \\
\hline Angle variation & 48 & 1830 & 38.1 & $<0.001$ \\
\hline Grain angle & 6 & 1600 & 266.8 & $<0.001$ \\
\hline Ring angle & 6 & 45 & 7.5 & $<0.001$ \\
\hline Grain x Ring & 36 & 184 & 5.1 & $<0.001$ \\
\hline Random error & 356 & 154 & 0.4 & \\
\hline Total & 404 & 1982 & & \\
\hline
\end{tabular}


Table B.2 - ANOVA tables for the orthotropic compression strength of Appalachian hardwoods $\left(\varphi>30^{\circ}\right)$

\begin{tabular}{|c|c|c|c|c|}
\hline \multicolumn{5}{|c|}{ ASPEN } \\
\hline Source & DF & Sum of Squares & Mean Square & $P$ value \\
\hline Angle variation & 34 & 1774 & 52.2 & $<0.001$ \\
\hline Grain angle & 4 & 1660 & 415.1 & $<0.001$ \\
\hline Ring angle & 6 & 39 & 6.4 & $<0.001$ \\
\hline Grain x Ring & 24 & 75 & 3.1 & $<0.001$ \\
\hline Random error & 134 & 133 & 0.989 & \\
\hline Total & 168 & 1906 & & \\
\hline \multicolumn{5}{|c|}{ RED OAK } \\
\hline Source & DF & Sum of Squares & Mean Square & P value \\
\hline Angle variation & 34 & 7064 & 207.8 & $<0.001$ \\
\hline Grain angle & 4 & 5802 & 1450.5 & $<0.001$ \\
\hline Ring angle & 6 & 675 & 112.5 & $<0.001$ \\
\hline Grain x Ring & 24 & 587 & 24.5 & $<0.001$ \\
\hline Random error & 135 & 259 & 1.916 & \\
\hline Total & 169 & 7323 & & \\
\hline \multicolumn{5}{|c|}{ YELLOW-POPLAR } \\
\hline Source & DF & Sum of Squares & Mean Square & $P$ value \\
\hline Angle variation & 34 & 4313 & 126.8 & $<0.001$ \\
\hline Grain angle & 4 & 4020 & 1005.1 & $<0.001$ \\
\hline Ring angle & 6 & 104 & 17.4 & $<0.001$ \\
\hline Grain x Ring & 24 & 188 & 7.8 & $<0.001$ \\
\hline Random error & 134 & 107 & 0.8 & \\
\hline Total & 168 & 4420 & & \\
\hline
\end{tabular}


Table B.3 - ANOVA tables for the orthotropic compression MOE of Appalachian hardwoods $\left(\varphi>30^{\circ}\right)$

\begin{tabular}{|c|c|c|c|c|}
\hline \multicolumn{5}{|c|}{ ASPEN } \\
\hline Source & DF & Sum of Squares & Mean Square & $P$ value \\
\hline Angle variation & 34 & 99.0 & 2.91 & $<0.001$ \\
\hline Grain angle & 4 & 75.2 & 18.81 & $<0.001$ \\
\hline Ring angle & 6 & 13.3 & 2.21 & $<0.001$ \\
\hline Grain x Ring & 24 & 10.5 & 0.44 & $<0.001$ \\
\hline Random error & 131 & 11.2 & 0.09 & \\
\hline Total & 165 & 110.3 & & \\
\hline \multicolumn{5}{|c|}{ RED OAK } \\
\hline Source & DF & Sum of Squares & Mean Square & $P$ value \\
\hline Angle variation & 34 & 121.2 & 3.86 & $<0.001$ \\
\hline Grain angle & 4 & 99.6 & 24.90 & $<0.001$ \\
\hline Ring angle & 6 & 17.8 & 2.97 & $<0.001$ \\
\hline Grain $x$ Ring & 24 & 13.8 & 0.58 & $<0.001$ \\
\hline Random error & 135 & 10.5 & 0.08 & \\
\hline Total & 169 & 141.8 & & \\
\hline \multicolumn{5}{|c|}{ YELLOW-POPLAR } \\
\hline Source & DF & Sum of Squares & Mean Square & $P$ value \\
\hline Angle variation & 34 & 137.5 & 4.04 & $<0.001$ \\
\hline Grain angle & 4 & 129.9 & 32.47 & $<0.001$ \\
\hline Ring angle & 6 & 2.3 & 0.39 & $<0.001$ \\
\hline Grain $x$ Ring & 24 & 5.3 & 0.22 & $<0.001$ \\
\hline Random error & 136 & 7.8 & 0.06 & \\
\hline Total & 170 & 145.3 & & \\
\hline
\end{tabular}


Table B.4 - ANOVA tables for the orthotropic dynamic MOE of Appalachian hardwoods

\begin{tabular}{|c|c|c|c|c|}
\hline \multicolumn{5}{|c|}{ ASPEN } \\
\hline Source & DF & Sum of Squares & Mean Square & P value \\
\hline Grain angle & 6 & $1.026 \times 10^{11}$ & $1.711 \times 10^{10}$ & $<0.001$ \\
\hline Specimen & 19 & $3.957 \times 10^{8}$ & $2.083 \times 10^{7}$ & 0.001 \\
\hline Random error & 114 & $9.236 \times 10^{8}$ & $8.102 \times 10^{6}$ & \\
\hline \multicolumn{5}{|c|}{$1.030 \times 10^{11}$} \\
\hline \multicolumn{5}{|c|}{ RED OAK } \\
\hline Source & DF & Sum of Squares & Mean Square & $P$ value \\
\hline Grain angle & 6 & $8.351 \times 10^{10}$ & $1.392 \times 10^{10}$ & $<0.001$ \\
\hline Specimen & 22 & $5.119 \times 10^{8}$ & $2.326 \times 10^{7}$ & 0.042 \\
\hline Random error & 132 & $1.846 \times 10^{8}$ & $1.399 \times 10^{7}$ & \\
\hline Total & 160 & $8.586 \times 10^{10}$ & & \\
\hline \multicolumn{5}{|c|}{ YELLOW-POPLAR } \\
\hline Source & DF & Sum of Squares & Mean Square & $P$ value \\
\hline Grain angle & 6 & $1.376 \times 10^{11}$ & $2.295 \times 10^{10}$ & $<0.001$ \\
\hline Specimen & 20 & $3.739 \times 10^{8}$ & $1.869 \times 10^{7}$ & 0.022 \\
\hline Random error & 120 & $1.212 \times 10^{9}$ & $1.010 \times 10^{7}$ & \\
\hline Total & 146 & $1.393 \times 10^{11}$ & & \\
\hline
\end{tabular}

Table B.5 - ANOVA table for the LVL bending measurements

\begin{tabular}{lrccc}
\hline \multicolumn{1}{c}{ Source } & DF & Sum of Squares & Mean Square & E(MS) \\
\hline Orientation & 1 & 0.184 & 0.184 & $\sigma^{2}{ }_{W}+5 \sigma_{B}^{2}+20 \kappa^{2}$ \\
Experimental error & 6 & 17.500 & 2.914 & $\sigma^{2}{ }_{W}+5 \sigma_{B}^{2}$ \\
Sampling error & 32 & 20.273 & 0.634 & $\sigma^{2}{ }_{W}$ \\
Total & 39 & 37.956 & & \\
\hline \hline & & & \\
& $\sigma^{2}{ }_{W}=0.634$ & $;$ & $\sigma^{2}{ }_{B}=\frac{2.914-0.634}{5}=0.456$
\end{tabular}


Table B.6 - ANOVA tables for the orthotropic shear strength of LVL and PSL (incomplete design)

\begin{tabular}{|c|c|c|c|c|}
\hline \multicolumn{5}{|c|}{ LVL } \\
\hline Source & DF & Sum of Squares & Mean Square & $P$ value \\
\hline Grain angle & 6 & 365.8 & 60.97 & $<0.001$ \\
\hline Ring angle & 6 & 169.9 & 28.32 & $<0.001$ \\
\hline Random error & 166 & 106.8 & 0.64 & \\
\hline Total & 178 & 865.8 & & \\
\hline \multicolumn{5}{|c|}{ RED OAK } \\
\hline Source & DF & Sum of Squares & Mean Square & $P$ value \\
\hline Grain angle & 6 & 503.8 & 83.96 & $<0.001$ \\
\hline Specimen & 6 & 89.3 & 14.89 & $<0.001$ \\
\hline Random error & 166 & 167.9 & 1.01 & \\
\hline Total & 178 & 878.4 & & \\
\hline
\end{tabular}

Table B.7 - ANOVA tables for the orthotropic compression MOE of LVL and PSL (incomplete design - log-transformed data)

\begin{tabular}{lrccc}
\hline \hline \multicolumn{5}{c}{ LVL } \\
Source & DF & Sum of Squares & Mean Square & P value \\
\hline Grain angle & 2 & 11.97 & 5.986 & $<0.001$ \\
Ring angle & 2 & 0.15 & 0.077 & $<0.001$ \\
Random error & 54 & 0.10 & 0.002 & \\
\hline Total & 58 & 17.22 & & \\
\hline \hline & & RED OAK & & P value \\
\hline Source & DF & Sum of Squares & Mean Square & $<0.001$ \\
\hline Grain angle & 2 & 12.17 & 6.086 & $<0.001$ \\
Specimen & 2 & 0.71 & 0.355 & \\
Random error & 54 & 0.16 & 0.003 & \\
\hline Total & 58 & 20.08 & & \\
\hline \hline
\end{tabular}




\section{APPENDIX C - SOME GEOMETRIC RELATIONSHIPS}

\section{C.1 Relationships between the grain angle of the shear load $(\vartheta)$, and the inclination of the sheared plane $(\Theta)$ for a compression specimen with certain grain and ring angle $(\varphi$ and $\theta)$ parameters.}

It can be shown that, using the notations of Figure C.1:

$$
\tan \alpha=\tan (\theta+\Theta) \sin \varphi
$$

If the specimen is placed in a three-dimensional cartesian coordinate system chosen so that the $z$-axis is parllel with the longitudinal axis of the specimen, the $x z$ plane includes the fiber direction and the lowest corner of the sheared plane corresponds to the origin, two vectors can be defined as follows (Figure C.2):

$$
\bar{X}\left(a, 0, \frac{a}{\tan \varphi}\right) ; \bar{Y}\left(0, b, \frac{b}{\tan \alpha}\right)
$$

The area of the sheared plane can be calculated as the length of the two vectors' vectorial product:

$$
\begin{aligned}
& \bar{X} \times \bar{Y}=\left|\begin{array}{ccc}
\bar{i} & \bar{j} & \bar{k} \\
a & 0 & \frac{a}{\tan \varphi} \\
0 & b & \frac{b}{\tan \alpha}
\end{array}\right|=\left(-\frac{a b}{\tan \varphi}\right) \bar{i}+\left(-\frac{a b}{\tan \alpha}\right) \bar{j}+(a b) \bar{k} \\
& A=\sqrt{\frac{(a b)^{2}}{\tan ^{2} \varphi}+\frac{(a b)^{2}}{\tan ^{2} \alpha}+(a b)^{2}}=a b \sqrt{1+\frac{1}{\tan ^{2} \varphi}+\frac{1}{\tan ^{2} \alpha}}
\end{aligned}
$$




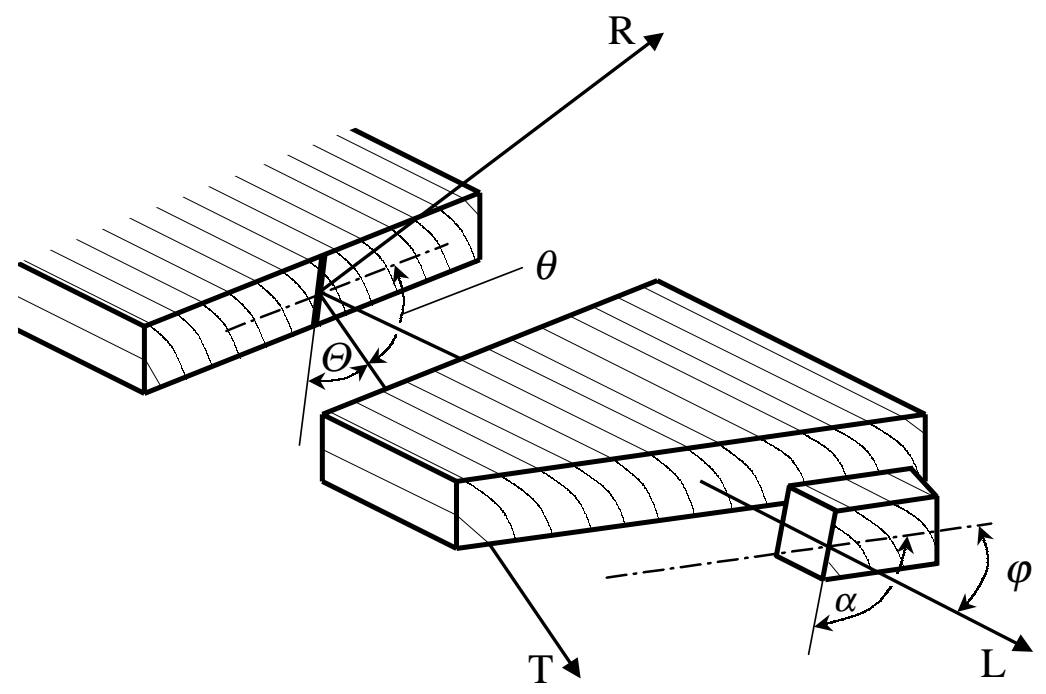

Figure C.1 - The definition of $\alpha$.

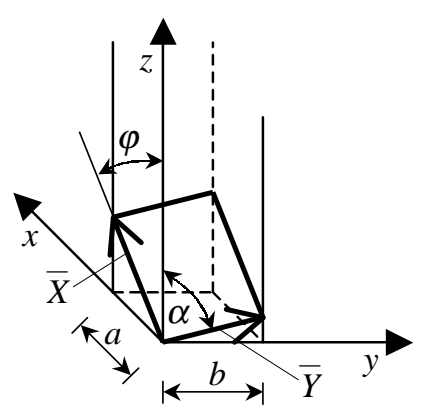

Figure C.2 - Vectors $\bar{X}$ and $\bar{Y}$

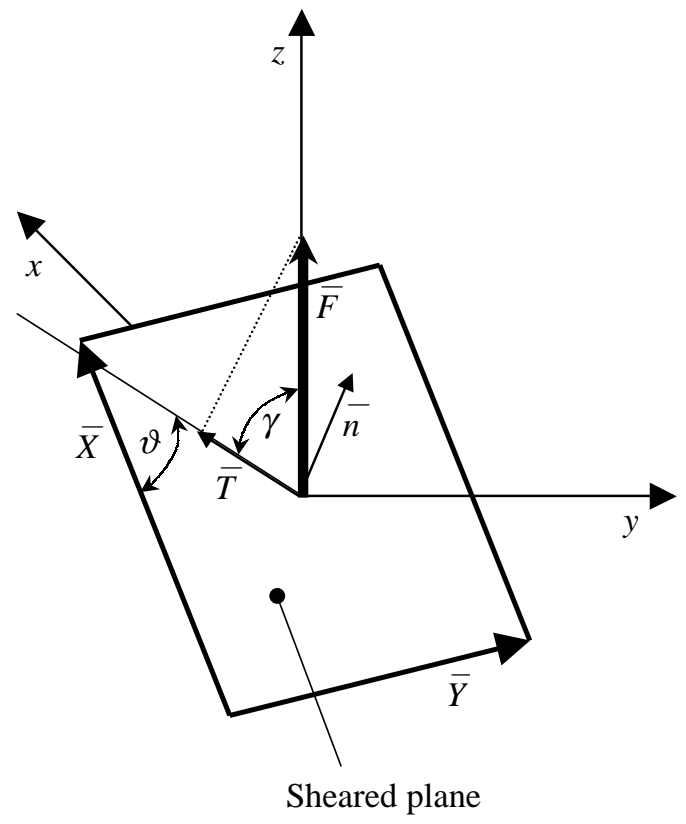

Figure C.3 - Shear conditions in the examined plane 
And a normal vector of the sheared plane can be defined as:

$$
\bar{n}\left(-\frac{1}{\tan \varphi},-\frac{1}{\tan \alpha}, 1\right)
$$

At the moment of failure, the vector of the applied force is $\bar{F}\left(0,0, F_{\max }\right)$. The vector $\bar{F}_{e}(0,0,1)$ points in the direction of the applied force. The angle between the load direction and the sheared plane $(\gamma-$ see Figure C.3 $)$ can be calculated as follows:

$$
\sin \gamma=\frac{\bar{n} \cdot \overline{F_{e}}}{|\bar{n}|\left|\overline{F_{e}}\right|}=\frac{1}{\sqrt{1+\frac{1}{\tan ^{2} \varphi}+\frac{1}{\tan ^{2} \alpha}}}
$$

The magnitude of the shear force the particular shear plane experiences will be:

$$
T=F_{\max } \cos \gamma
$$

A direction vector parallel to the shear load can be found by multiplying $\bar{F}_{e}$ with $\bar{n}$ twice, vectorially.

$$
\begin{array}{r}
\overline{F_{e}} \times \bar{n}=\left|\begin{array}{ccc}
\bar{i} & \bar{j} & \bar{k} \\
0 & 0 & 1 \\
-\frac{1}{\tan \varphi} & -\frac{1}{\tan \alpha} & 1
\end{array}\right|=\frac{1}{\tan \alpha} \bar{i}-\frac{1}{\tan \varphi} \bar{j} \\
\bar{t}=\overline{F_{e} \times n} \times \bar{n}=\left|\begin{array}{ccc}
\bar{i} & \bar{j} & \bar{k} \\
\frac{1}{\tan \alpha} & -\frac{1}{\tan \varphi} & 0 \\
-\frac{1}{\tan \varphi} & -\frac{1}{\tan \alpha} & 1
\end{array}\right|=-\frac{1}{\tan \varphi} \bar{i}-\frac{1}{\tan \alpha} \bar{j}-\left(\frac{1}{\tan ^{2} \varphi}+\frac{1}{\tan ^{2} \alpha}\right) \bar{k}
\end{array}
$$


Finally, the angle $(\vartheta)$ between the grain direction $(\bar{X})$ and the shear load is

$$
\cos \vartheta=\frac{\bar{t} \cdot \bar{X}}{|\bar{t}||\bar{X}|}=\frac{-\frac{a}{\tan \varphi}-\frac{a}{\tan \varphi}\left(\frac{1}{\tan ^{2} \alpha}+\frac{1}{\tan ^{2} \varphi}\right)}{\sqrt{a^{2}+\frac{a^{2}}{\tan ^{2} \varphi}} \sqrt{\frac{1}{\tan ^{2} \alpha}+\frac{1}{\tan ^{2} \varphi}+\left(\frac{1}{\tan ^{2} \alpha}+\frac{1}{\tan ^{2} \varphi}\right)^{2}}}
$$

After full simplification, the above formula reduces to the following:

$$
\cos \vartheta=\cos \varphi \frac{\sqrt{\frac{1}{\tan ^{2} \alpha}+\frac{1}{\sin ^{2} \varphi}}}{\sqrt{\frac{1}{\tan ^{2} \alpha}+\frac{1}{\tan ^{2} \varphi}}}
$$

The maximum shear stress in the cross-section can be calculated by dividing the shear load by the cross sectional area at the time of failure.

\section{C.2 Derivation of the grain and ring angle $\left(\varphi_{i}\right.$ and $\left.\theta_{i}\right)$ of a load with certain load and strand orientation ( $\varphi^{\prime}$ and $\left.\theta^{\prime}\right)$ acting on a PSL strand with certain strand angle and strand deviation $\left(\alpha_{i}\right.$ and $\left.\beta_{i}\right)$}

On Figure C.4 vectors $\bar{B}$ and $\bar{F}$ are the direction unit vectors of the orieintation and the load. By definition, $\bar{B}$ is in the $y z$ plane, and the anatomical direction vector $\bar{L}$ is in the $x y$ plane. Vector $\bar{F}$ is general. In the $x y z$ coordinate system, these unit vectors have the following coordinates:

$$
\begin{gathered}
\bar{B}\left(0, \cos \beta_{i}, \sin \beta_{i}\right) \\
\bar{L}\left(\cos \alpha_{i}, \sin \alpha_{i}, 0\right) \\
\bar{F}\left(\cos \varphi^{\prime}, \sin \varphi^{\prime} \cos \theta^{\prime}, \sin \varphi^{\prime} \sin \theta^{\prime}\right)
\end{gathered}
$$




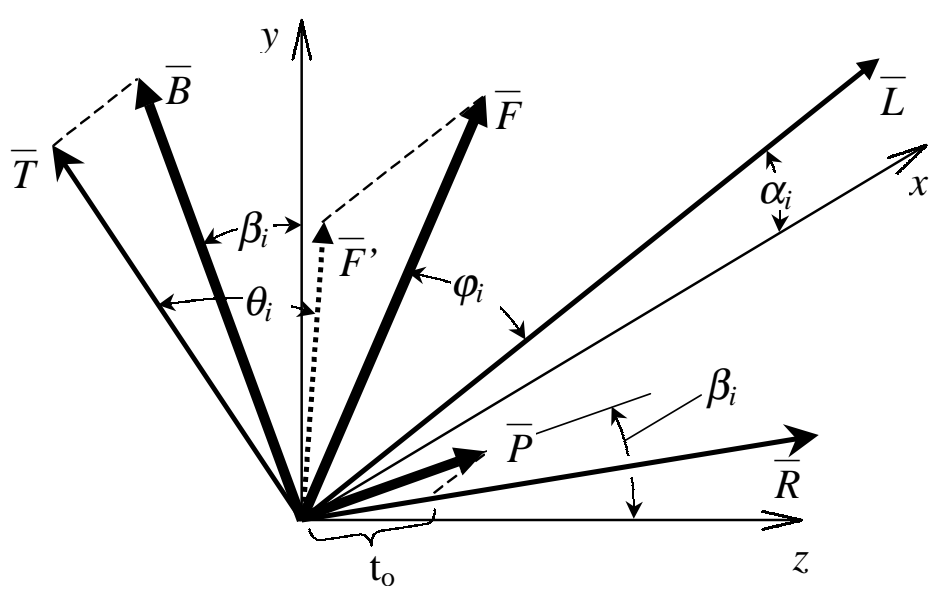

Figure C.4 - Definitions of different vectors and angles

$\varphi_{i}$ and $\theta_{i}$ are defined as the angle between $\bar{L}$ and $\bar{F}$ and the angle between the vectors $\bar{F}$, (the projection of $\bar{F}$ on the RT plane), and $\bar{T}$, respectively. The angle $\varphi_{i}$ can be calculated as follows:

$$
\cos \varphi_{i}=\frac{\bar{F} \cdot \bar{L}}{|\bar{F}||\bar{L}|}=\cos \varphi^{\prime} \cos \alpha_{i}+\sin \varphi^{\prime} \sin \alpha_{i} \cos \theta^{\prime}
$$

For the calculation of $\theta_{i}$ the coordinates of the $\bar{F}$, and the $\bar{T}$ vectors need to be calculated. The endpoints of these vectors can be found by perpendicular projection onto the RT plane. The normal vector of RT is $\bar{L}$, and the plane includes the origin, thus the equation of the plane is:

$$
x \cos \alpha_{i}+y \sin \alpha_{i}=0
$$

The parametric equations of a line that goes through the endpoint of $\bar{B}$ and is parallel with $\bar{L}$ (i.e. perpendicular to $\mathrm{RT}$ ) are: 


$$
\begin{aligned}
& x=t \cos \alpha_{i} \\
& y=\cos \beta_{i}+t \sin \alpha_{i} \\
& z=\sin \beta_{i}
\end{aligned}
$$

Substituting these into the equation of RT, we get

$$
\begin{aligned}
& t \cos ^{2} \alpha_{i}+t \sin ^{2} \alpha_{i}+\sin \alpha \cos \beta_{i}=0 \\
& t=-\sin \alpha_{i} \cos \beta_{i}
\end{aligned}
$$

which yields the following coordinates for $\bar{T}$ :

$$
\bar{T}\left(-\cos \alpha_{i} \sin \alpha_{i} \cos \beta_{i}, \cos ^{2} \alpha_{i} \cos \beta_{i}, \sin \beta_{i}\right)
$$

A similar line that goes through the endpoint of $\bar{F}$ is

$$
\begin{aligned}
& x=\cos \varphi^{\prime}+t \cos \alpha_{i} \\
& y=\sin \varphi^{\prime} \cos \theta^{\prime}+t \sin \alpha_{i} \\
& z=\sin \varphi^{\prime} \sin \theta^{\prime}
\end{aligned}
$$

After substitution and simplification this yields the following coordinates for $\bar{F}$ :

$\bar{F}^{\prime}\left(\sin \alpha_{i}\left(\cos \varphi^{\prime} \sin \alpha_{i}-\sin \varphi^{\prime} \cos \alpha_{i} \cos \theta^{\prime}\right), \sin \varphi^{\prime} \sin \theta^{\prime}, \cos \alpha_{i}\left(-\cos \varphi^{\prime} \sin \alpha_{i}+\sin \varphi^{\prime} \cos \alpha_{i} \cos \theta^{\prime}\right)\right)$

After full simplification, the cosine of $\theta_{i}$ is given as:

$$
\cos \theta_{i}=\frac{\bar{F}^{\prime} \cdot \bar{T}}{\left|\bar{F}^{\prime}\right||\bar{T}|}=\frac{\cos \alpha_{i} \cos \beta_{i} \Gamma+\sin \beta_{i} \sin \varphi^{\prime} \sin \theta^{\prime}}{\sqrt{\cos ^{2} \alpha_{i} \cos ^{2} \beta_{i}+\sin ^{2} \beta_{i}} \sqrt{\Gamma^{2}+\sin ^{2} \varphi^{\prime} \sin ^{2} \theta^{\prime}}}
$$

where $\Gamma=\sin \varphi^{\prime} \cos \alpha_{i} \cos \theta^{\prime}-\cos \varphi^{\prime} \sin \alpha_{i}$.

Since the length of $\bar{T}$ is $\sqrt{\cos ^{2} \alpha_{i} \cos ^{2} \beta_{i}+\sin ^{2} \beta_{i}}$, when the length of $\bar{B}$ is one, this is the quantity by which we need to divide the actual strand width to get the projected strand width on the $y z$ plane (i.e. on the beam cross-section.) 
The real strand thickness can be calculated from the projected strand thickness by defining the projected strand thickness vector: $\bar{T}_{P}\left(0, t_{p} \sin \beta_{i}, t_{p} \sin \beta_{i}\right)$, where $t_{p}$ is the projected thickness. The length of projection of this vector on the RT plane is the real thickness.

The equations of a line that goes through the endpoint of $\bar{T}_{P}$ and is parallel with $\bar{L}$ are:

$$
\begin{aligned}
& x=t \cos \alpha_{i} \\
& y=t_{P} \sin \beta_{i}+t \sin \alpha_{i} \\
& z=t_{P} \cos \beta_{i}
\end{aligned}
$$

Substituting the above values into the plane equation yields $t=-t_{P} \sin \beta_{i} \sin \alpha_{i}$. The coordinates of the real thickness vector are, accordingly:

$$
\bar{T}_{o}\left(-t_{P} \sin \beta_{i} \sin \alpha_{i} \cos \alpha_{i}, t_{P} \sin \beta_{i} \cos ^{2} \alpha_{i}, t_{P} \cos \beta_{i}\right)
$$

the original thickness is calculated as the length of this vector:

$$
t_{o}=t_{P} \sqrt{\sin ^{2} \beta_{i} \cos ^{2} \alpha_{i}+\cos ^{2} \beta_{i}}
$$




\section{APPENDIX D - CALCULATION OF THE CORRECTION FACTOR FOR THE DENSIFICATION EFFECT}

The following computation was used to calculate the modification factor for the densification effect:

$$
f=\frac{\lim _{\Delta \rho_{\min } \rightarrow 0}\left(\int_{\Delta \rho_{\min }}^{\Delta \rho_{\max }} \frac{\Delta E_{\text {stat }}}{\Delta E_{d y n}} \frac{\Delta \rho}{\Delta \rho_{\max } / 2} d \Delta \rho\right)}{\Delta \rho_{\max }-\Delta \rho_{\min }}
$$

where: $f \quad-$ the factor by which the $\Delta E_{d y n}$ needs to be divided;

$\Delta E_{\text {stat }}, \Delta E_{d y n}$ - the quadratic MOE increase function fitted on the dynamic and static MOE increase values, respectively;

$\Delta \rho_{\max }, \Delta \rho_{\min }-$ the limits of the densification range over which the factor is calculated.

The integration contains the ratio of the static and dynamic MOE increase. This ratio, which is a function of $\Delta \rho$, was integrated within the range of typical densification values used in the simulation $\left(0 \% \sim 120 \%\right.$.) Since $\Delta \mathrm{E}_{\mathrm{dyn}}$ is zero at $\Delta \rho=0$, the ratio is not defined at this point, and a limit function was used to approach this value. Furthermore, since both $\Delta E_{\text {stat }}$ and $\Delta E_{d y n}$ increase with $\Delta \rho$, the ratio is more important at higher densification levels. Therefore, it is weighted with densification. Finally, the calculated integral is divided by the length of the integration range, to calculate the weighted average ratio. 


\section{APPENDIX E - Program DOCUMENTATION}

\section{E.1 Simulation programs}

The following sections introduce the four main simulation routines. The programs are fairly flexible in that the layup of LVL as well as the species mix used in PSL can be specified arbitrarily. Species-specific information is stored in the Species module (see section E.2) which can be expanded to include further species. Certain calculations and simulations are executed by calling the functions described in section E.3.

Important notice: the simulation routines as well as some of the functions use external functions from the statistical IMSL libraries. The name of these libraries must be specified in the project settings.

\section{The LVLBend program}

Figure E.1 shows the flowchart for the program LVLBend, which simulates the bending MOE of LVL. This flowchart, with the code included below clarifies the operation of the program almost fully. The array S contains the species layup from the compression to the tension side of the beam, coded by integer numbers. The species code is 1 for quaking aspen, 2 for red oak and 3 for yellow-poplar.

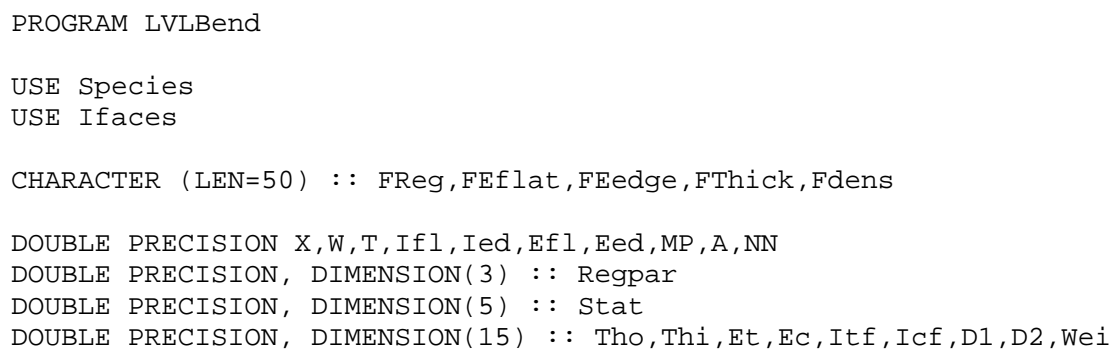




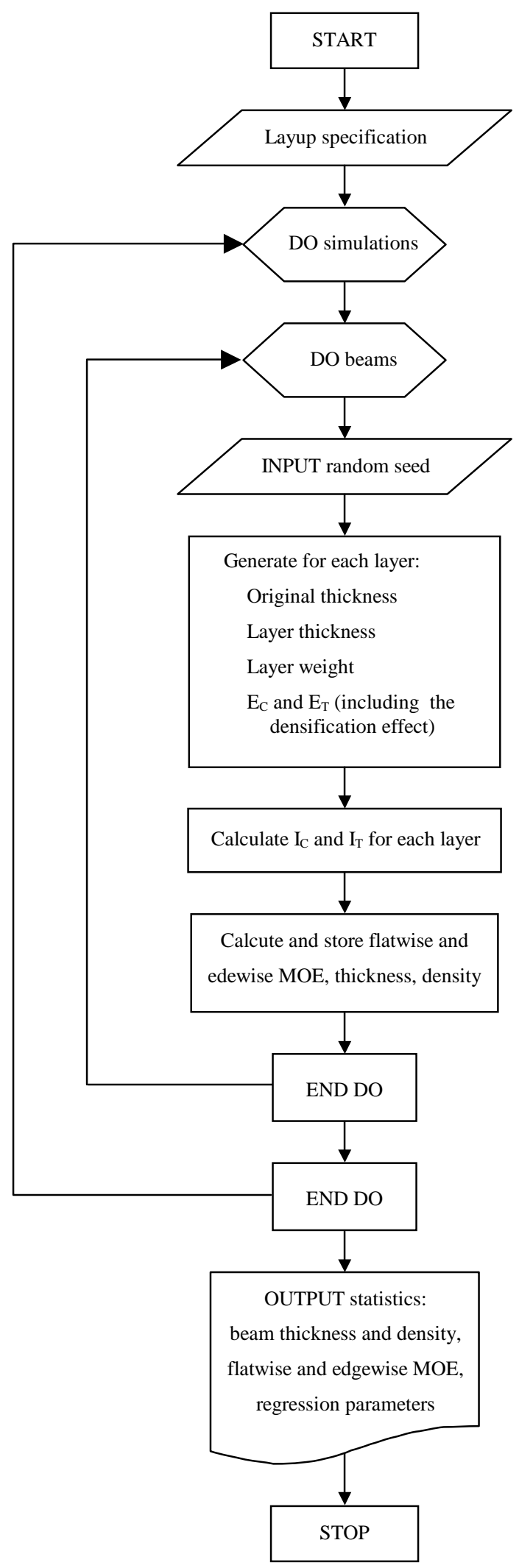

Figure E.1 - Flowchart of the program LVLBend 


\section{(Program LVLBend continued)}

DOUBLE PRECISION, DimENSION(:,:), ALLOCATABLE :: Ee,Ef, Th, Dy

EXTERNAL RNSET, RNNOF, RNUNF

INTEGER Iseed, I, J, N, Q, P, M

INTEGER, DIMENSION(15) : : S

$\mathrm{M}=20$ ! Number of Monte-Carlo Simulations

$\mathrm{N}=20$ ! Number of beams per simulation

! The names of the files to contain regressional info. and statistics for

! edgewise and flatwise MOE, thickness and density of the simulated beams

FReg="C: \simres $\backslash$ LVLbend_Reg.txt"

FEedge $=" C: \backslash$ simres $\backslash$ LVLbend_EDGE.txt"

FEflat $=" C: \backslash$ simres $\backslash$ LVLbend_FLAT.txt"

FThick="C: $\backslash$ simres $\backslash$ LVLbend_THICK.txt"

FDens="C: \simres $\backslash$ LVLbend_DENS.txt"

$\mathrm{W}=5 \quad$ ! Beam width (does not effect simulation results.)

$S=(/ 3,3,3,3,3,3,3,3,3,3,3,3,3,3,3 /) \quad !$ The layup

ALLOCAte ( Ee $(M, N), \operatorname{Ef}(M, N), \operatorname{Th}(M, N), \operatorname{Dy}(M, N))$

OPEN (UNIT=100, STATUS="OLD", file="c $: \backslash$ My documents $\backslash$ fortran $\backslash$

\&ranstream.txt")

! ranstream.txt contains a streem of random integers between 0 and 10000 .

DO $Q=1, M \quad$ ! Executes $M$ number of simulations

WRITE $(*$, fmt $="(I 2, T 13, \backslash) "), Q$

DO $\mathrm{J}=1, \mathrm{~N}$ ! Generates $\mathrm{N}$ beams for each simulation

! Reading and initializing the random seed

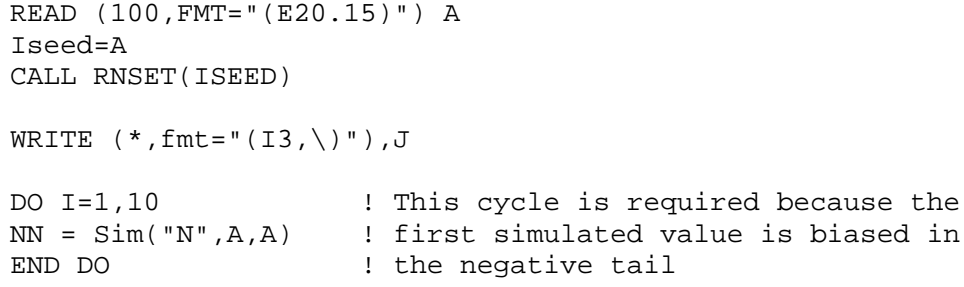

! Generating layer properties

DO $I=1,15$

Tho $(I)=\operatorname{Orig}(S(I))$

Wei $(I)=$ Tho $(I) * \operatorname{Dty}(S(I))$

SELECT CASE (I)

CASE $(1,2,14,15)$

Thi (I) $=$ Face (Tho (I)) 
(Program LVLBend contiunued)

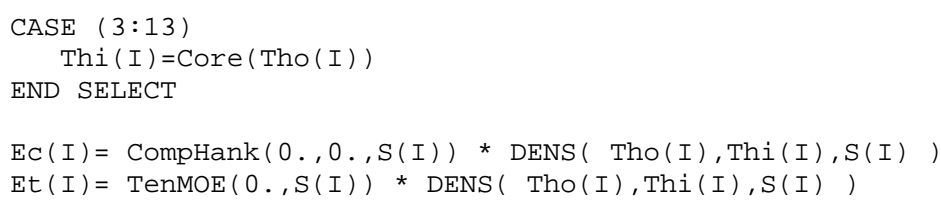

! Storing the flatwise and edgewise MOE, beam thickness and density

$\operatorname{Ee}(Q, J)=E e d$

$\operatorname{Ef}(Q, J)=\operatorname{Efl}$

$\operatorname{Th}(Q, J)=T$

$\operatorname{Dy}(Q, J)=\operatorname{SUM}($ Wei $) / T$

END DO ! Ends ' $\mathrm{N}$ '

PRINT *, " " 
(Program LVLBend continued)

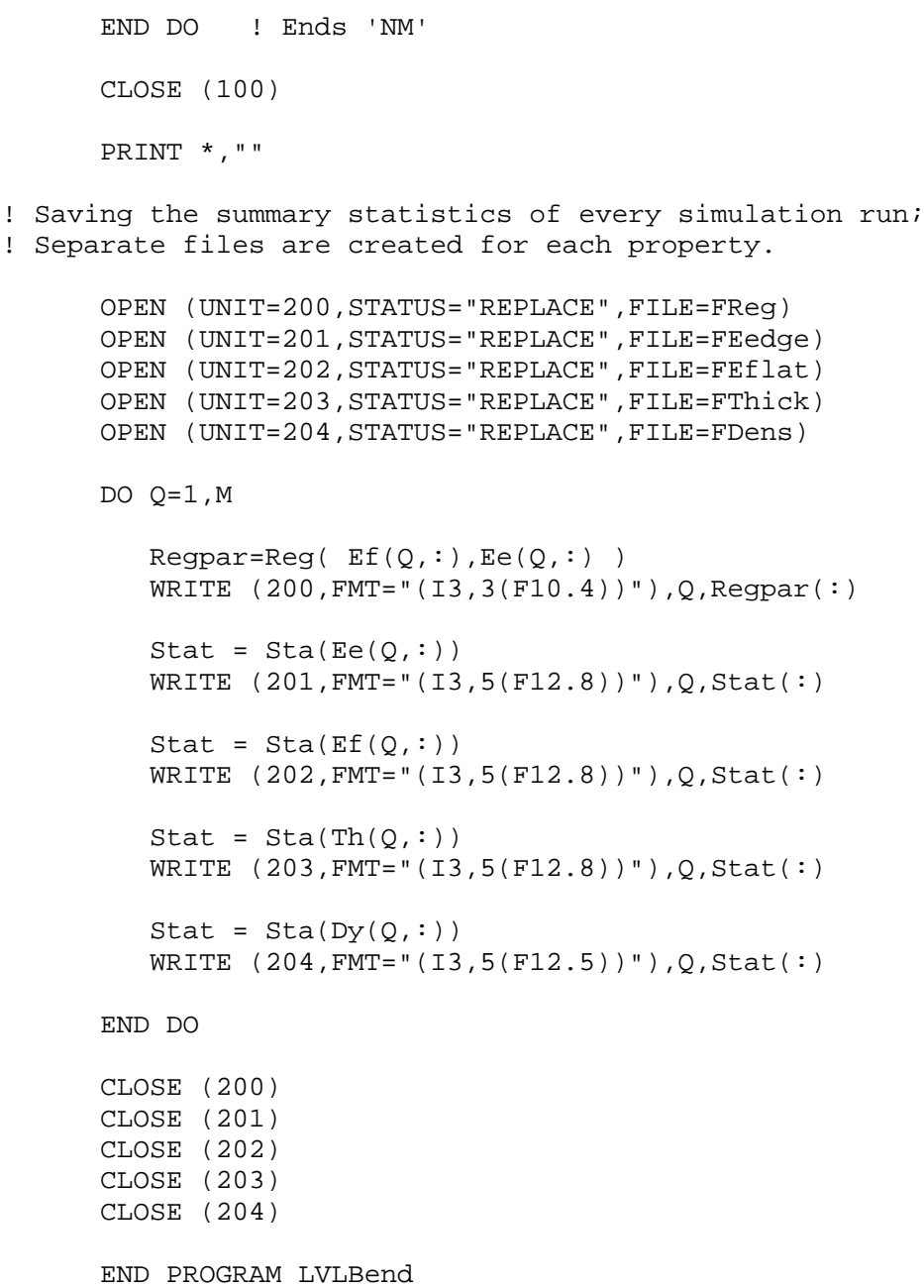

\section{The PSLBend program}

Figure E.2 shows the flowchart for the program P SLBend, which simulates the bending MOE of PSL. This program is fairly similar to LVLBend, but is somewhat more complex, includes more random variables. Most notably, the calculation of the $I_{i}$ values is more complicated, involving numerical integration. Instead of a layup, a species mix is specified. The first number in the variable Smix is the proportion of the species denoted by species code 1, the 


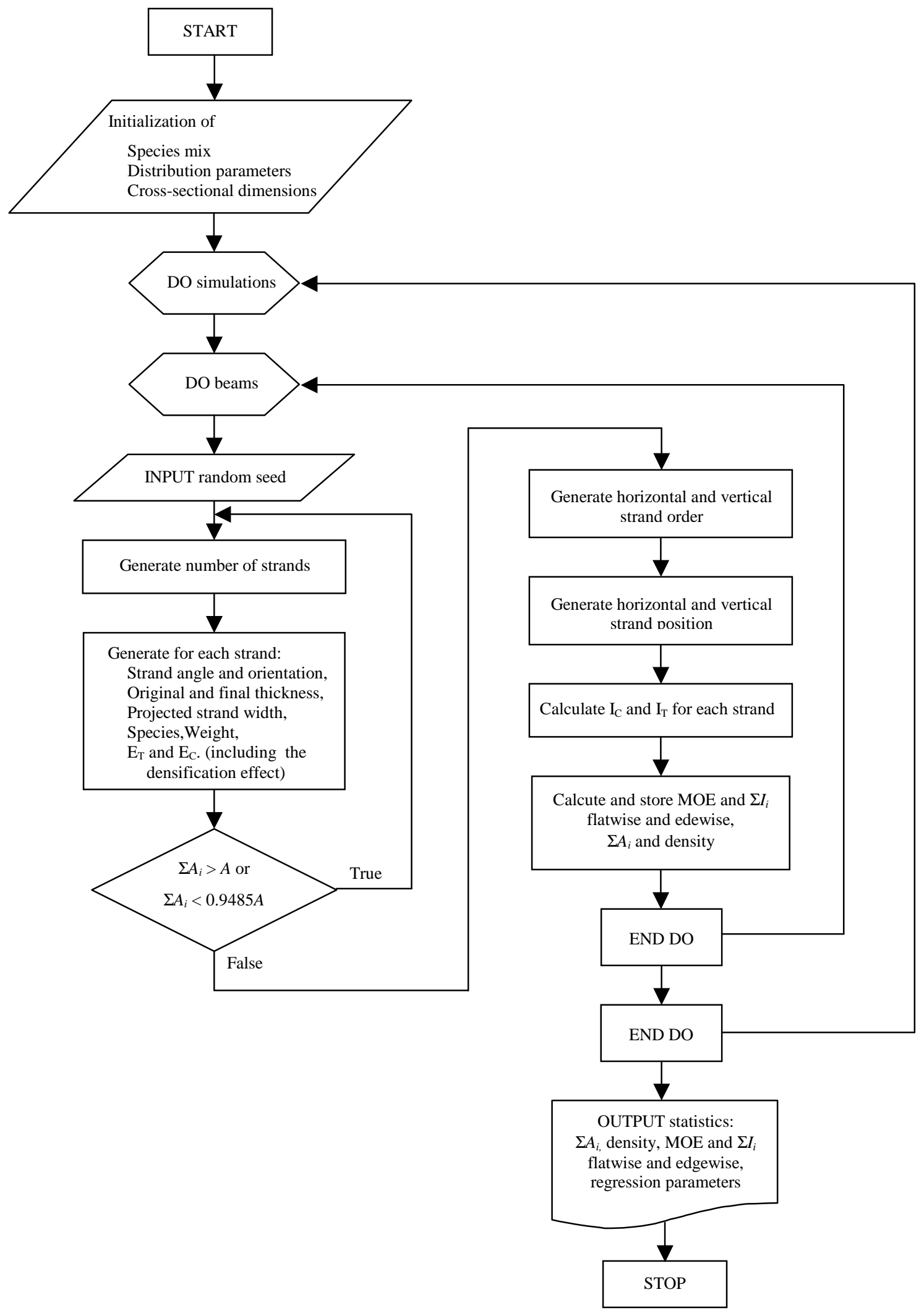

Figure E.2 - Flowchart of the program P SLBend 
Table E.1 - The species-specific parameters specified in the Species module

\begin{tabular}{ll}
\hline \hline Name & \\
\hline Am & Mean strand angle \\
As & Standard deviation of strand angle \\
Fm & Average number of flakes per in ${ }^{2}$ \\
Fs & Standard deviation of number of flakes per in ${ }^{2}$ \\
Om & Mean strand deviation \\
Os & Standard deviation of strand deviation \\
Tm & Mean final strand thickness (in) \\
Ts & Standard deviation of final strand thickness (in) \\
\hline \hline
\end{tabular}

second is that coded 2, etc. (e.g. for a pure yellow-poplar beam, Smix can contain the numbers $\{0,0,100\}$, since the code of yellow-poplar is 3.)

The parameters of the normal distributions for simulating the geometric parameters of the constituents are specified in the variables shown in Table E.1. $R$ is the resolution of the numerical integration - increasing it makes the simulation more accurate but longer to run.

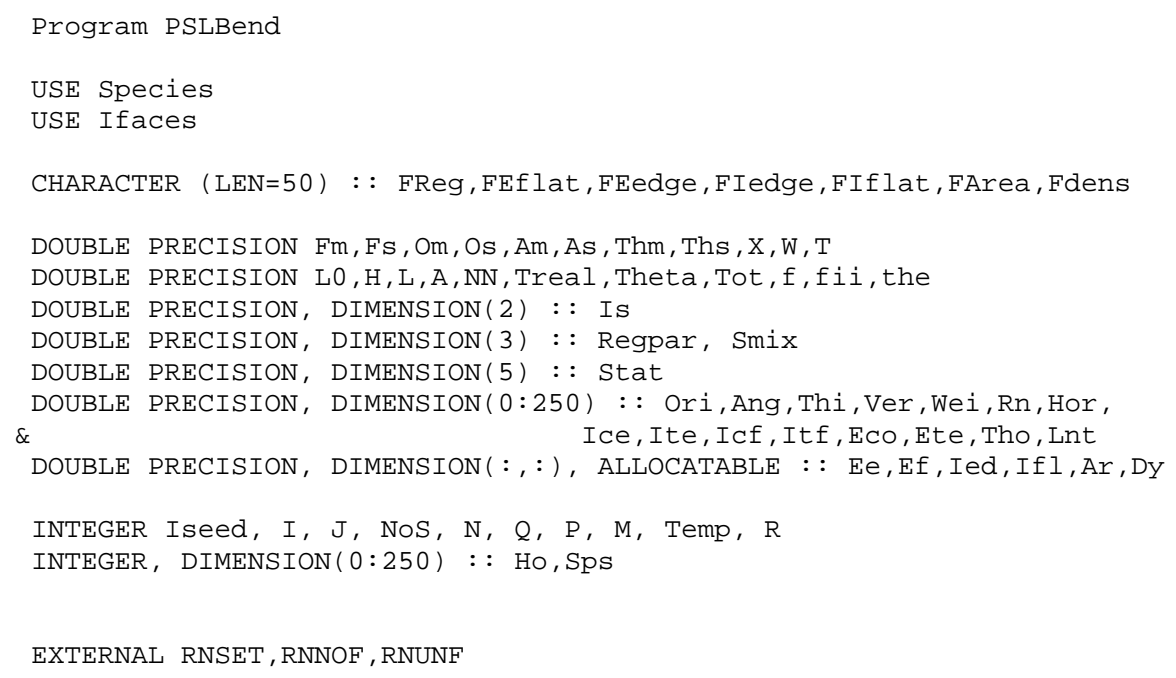




\section{(Program PSLBend continued)}

1

$\mathrm{NN}=\mathrm{W}^{*} \mathrm{H}{ }^{*} \mathrm{Sim}(" \mathrm{~N} ", \mathrm{Fm}, \mathrm{Fs})$ ! Simulates the number of strands

NoS $=$ INT $(\mathrm{NN}) \quad$ ! Integer part (full width strands)

$\mathrm{LO}=\mathrm{NN}-\mathrm{NoS} \quad$ ! Fractional part (width of strand nr 0. )

! Simulation of strand parameters

DO $I=0$, NOS

Ori (I) = Sim( "N", Om, Os )

Ang $(I)=\operatorname{Sim}(" \mathrm{~N} ", \mathrm{Am}, \mathrm{As})$

$\operatorname{Sps}(I)=$ SChoose $(\operatorname{Smix})$

Lnt $(I)=1 / \operatorname{SQRT}(\operatorname{COSD}(\operatorname{Ang}(I)) * * 2 * \operatorname{COSD}(\operatorname{Ori}(\mathrm{I})) * * 2+\operatorname{SIND}(\operatorname{Ori}(\mathrm{I})) * * 2)$

Tho (I) $=\operatorname{Orig}(\operatorname{Sps}(\mathrm{I}))$

Thi $(I)=\operatorname{Sim}(" \mathrm{~N} ", \mathrm{Thm}, \mathrm{Ths})$

Wei $(I)=\operatorname{Tho}(I) * \operatorname{Lnt}(I) * \operatorname{Dty}(\operatorname{Sps}(I))$

$\operatorname{Treal}=\operatorname{THI}(I) * \operatorname{SQRT}(\operatorname{SIND}(\operatorname{Ori}(I)) * \star 2 * \operatorname{COSD}(\operatorname{Ang}(I)) * \star 2+\operatorname{COSD}(\operatorname{Ori}(\mathrm{I})) * \star 2)$

IF (Treal>Tho(I)) THEN ! This makes sure that the final thickness is GO TO 5 ! Smaller than the original.

END IF

Fii=Ang $(I)$

The=Theta (Ang (I), Ori (I), 0.,0.)

$\operatorname{Eco}(I)=$ CompHank (Fii, The, Sps (I)) *Dens (Tho(I), Treal, Sps (I))

Ete $(I)=$ TenMoE (Fii,Sps (I)) *Dens (Tho(I), Treal, Sps (I))

END DO

Lnt $(0)=\operatorname{Lnt}(0) * \mathrm{~L} 0 \quad$ ! The length and weight of the first strand

Wei $(0)=$ Wei $(0) *$ L0 ! Is decreased according to L0

Tot $=$ SUM $($ Thi $(0:$ NoS $) *$ Lnt $(0:$ NoS $)) \quad$ ! The combined strand cross-section

! Beam rejections based on the combined cross-section

IF $\left(\right.$ Tot $\left.>H^{*} W\right)$ THEN

GO TO 1

ELSE IF $($ Tot $<0.95 * H * W)$ THEN

GO TO 1

END IF

! Simulation of the horizontal order (vertical order is unchanged)

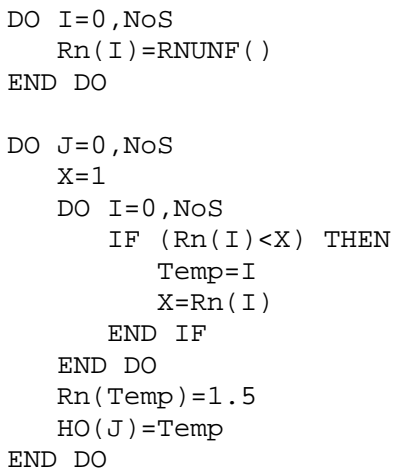




\section{(Program PSLBend continued)}

! Simulation of the horizontal and vertical position

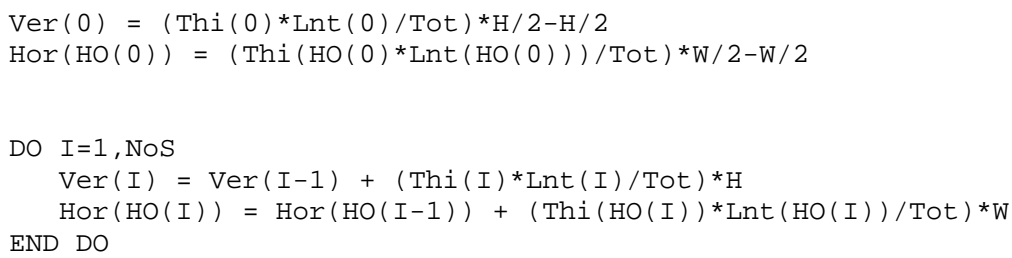

! Calculation of moment of inertia values horizontally and vertically

DO $I=0$, NoS

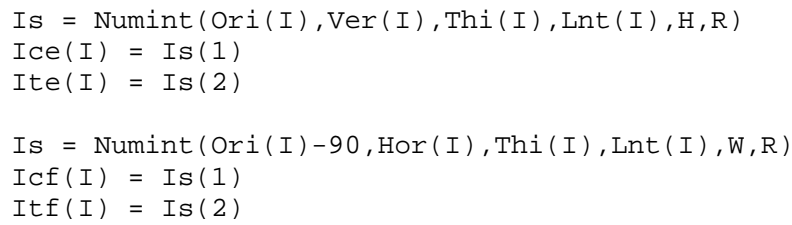

END DO

! Calculating and storing flatwise and edgewise MOE and combined

! moment of inertia, the combined cross-section of the strands

! and beam density

$\operatorname{Ee}(Q, P)=\operatorname{SUM}(\operatorname{Ice}(0: \operatorname{NoS}) \star \operatorname{Eco}(0: \operatorname{NoS})+\operatorname{Ite}(0: \operatorname{NoS}) \star \operatorname{Ete}(0: \operatorname{NoS})) /\left(H^{\star} \star 3 * W / 12\right)$ $\operatorname{Ef}(Q, P)=\operatorname{SUM}(\operatorname{ICf}(0: \operatorname{NoS}) \star \operatorname{ECO}(0: \operatorname{NoS})+\operatorname{Itf}(0: \operatorname{NoS}) \star \operatorname{Ete}(0: \operatorname{NoS})) /\left(H^{\star} W^{\star} \star 3 / 12\right)$

$\operatorname{Ied}(Q, P)=\operatorname{SUM}(\operatorname{Ice}(0: \operatorname{NoS})+\operatorname{Ite}(0: \operatorname{NoS}))$

$\operatorname{Ifl}(Q, P)=\operatorname{SUM}(\operatorname{ICf}(0: \operatorname{NoS})+\operatorname{Itf}(0:$ NoS $))$

$\operatorname{Ar}(Q, P)=$ Tot

$\operatorname{Dy}(Q, P)=\operatorname{SUM}($ Wei $(0:$ NoS $)) /\left(W^{\star} H\right)$

END DO ! Ends ' $\mathrm{N}$ '

print *," "

END DO ! Ends ' $M$ '

CLOSE (100)

print *," "

! Saving the summary statistics of every simulation run;

! Separate files are created for each property.

OPEN (UNIT=200, STATUS="REPLACE ", FILE=FReg)

OPEN (UNIT=201, STATUS="REPLACE", FILE=FEedge)

OPEN (UNIT=202, STATUS="REPLACE", FILE=FEflat)

OPEN (UNIT=203, STATUS="REPLACE", FILE=FIedge)

OPEN (UNIT=204, STATUS="REPLACE", FILE=FIflat)

OPEN (UNIT=205, STATUS="REPLACE" , FILE=FArea)

OPEN (UNIT=206, STATUS="REPLACE", FILE=FDens ) 
(Program PSLBend continued)

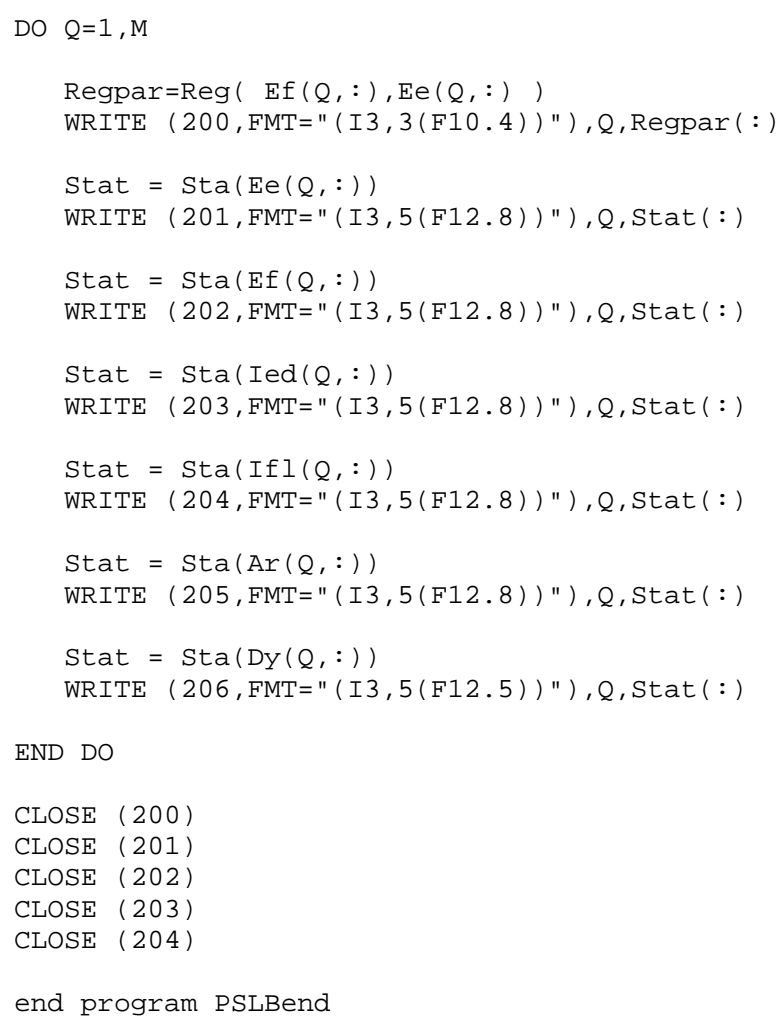

\section{$\underline{\text { The LVLComp program }}$}

Figure E.3 shows the flowchart for the program LVLComp, which simulates the orthotropic compression MOE of LVL in a certain direction. The program is similar to, though simpler than LVLBend. The variables Fip and Thp contain the $\varphi^{\prime}$ and $\theta^{\prime}$ values associated with the compression load, respectively. The program outputs only the statistics for the simulated compression MOE.

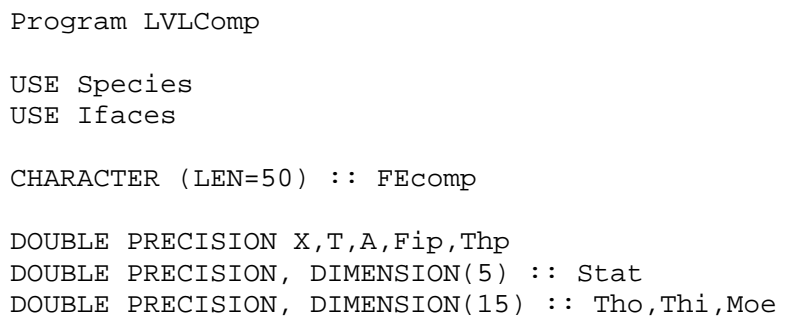




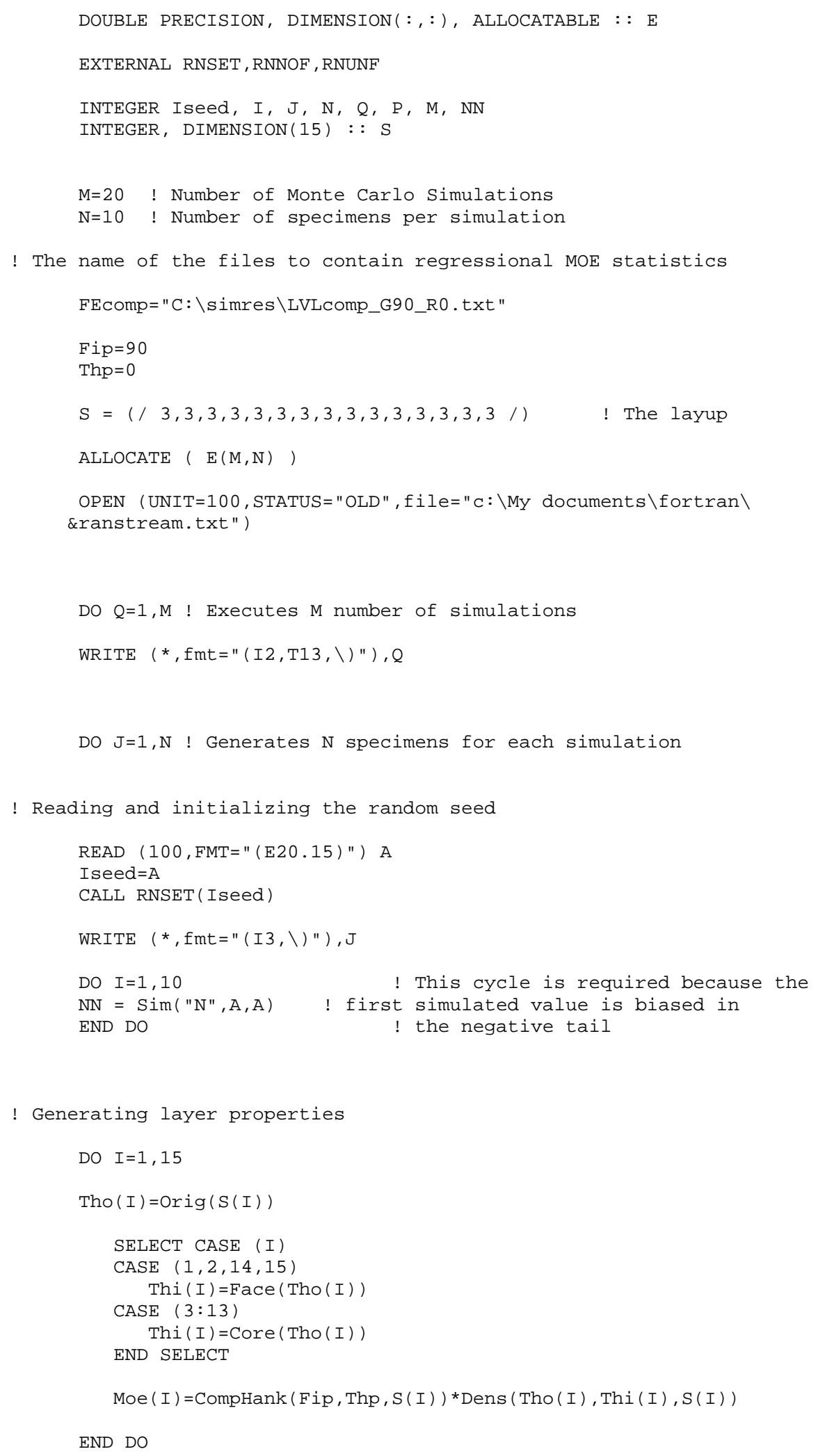




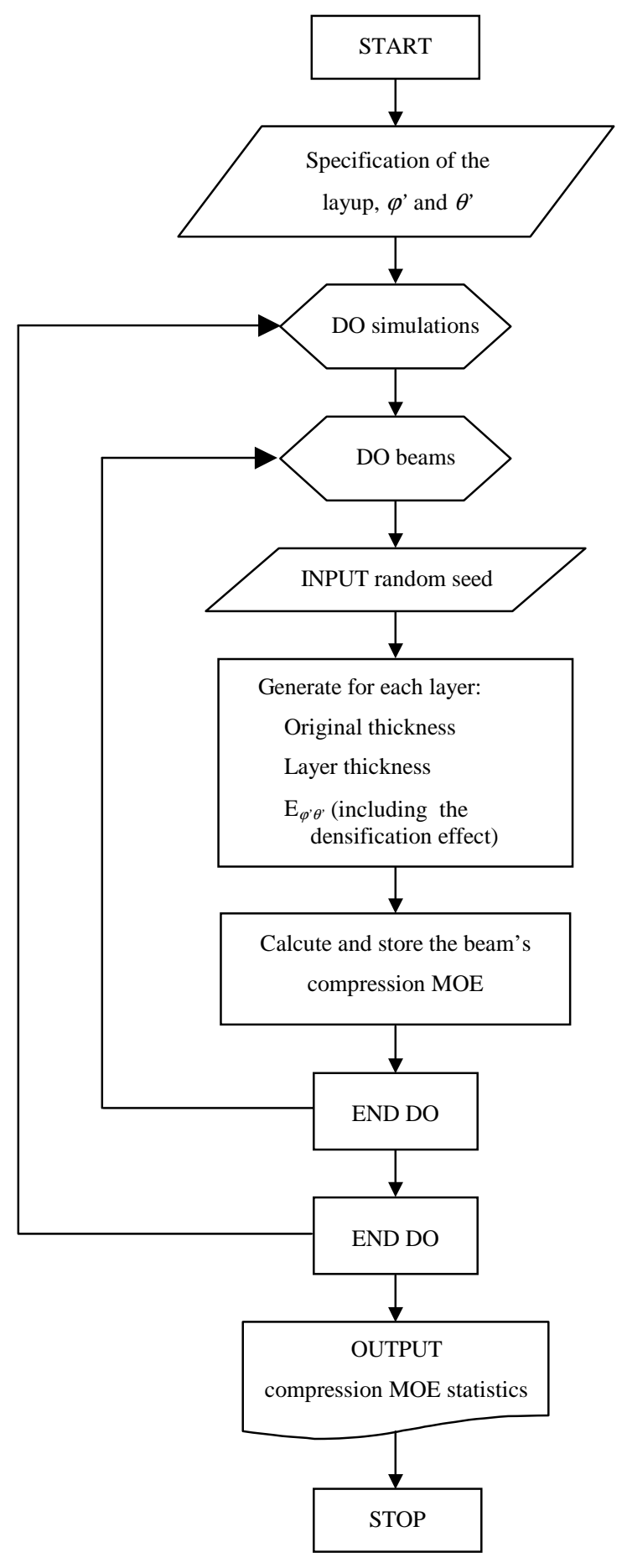

Figure E.3 - Flowchart of the program LVLComp 
(Program LVLComp continued)

! Calculating beam thickness and compression MOE

$\mathrm{T}=\mathrm{SUM}(\mathrm{THI})$

$\mathrm{E}(\mathrm{Q}, \mathrm{J})=\mathrm{SUM}(\mathrm{MOE} * \mathrm{THI}) / \mathrm{T}$

END DO ! Ends ' $\mathrm{N}$ '

print *, " "

END DO ! Ends 'NM'

CLOSE (100)

print *, " "

(Program LVLComp continued)

OPEN (UNIT=200, STATUS="REPLACE" , FILE=FECOmp)

DO $Q=1, M$

Stat $=\operatorname{Sta}(E(Q,:))$

WRITE (200, FMT=" (I3,5(F12.8)) "), Q, Stat (:)

END DO

CLOSE (200)

end program LVLComp

The PSLComp program

Figure E.4 shows the flowchart for the program PSLComp, which simulates the orthotropic compression MOE of PSL in a given direction. See some remarks at P SLbend and LVLComp.

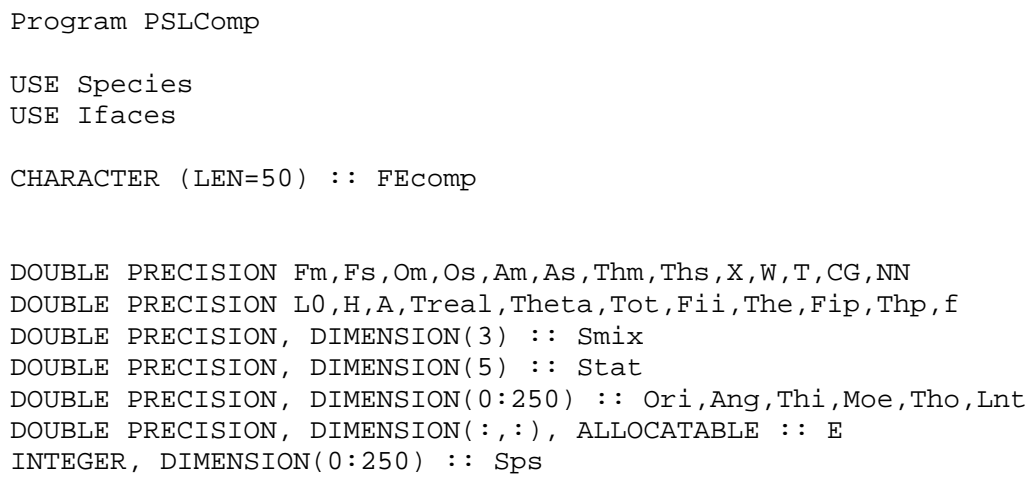




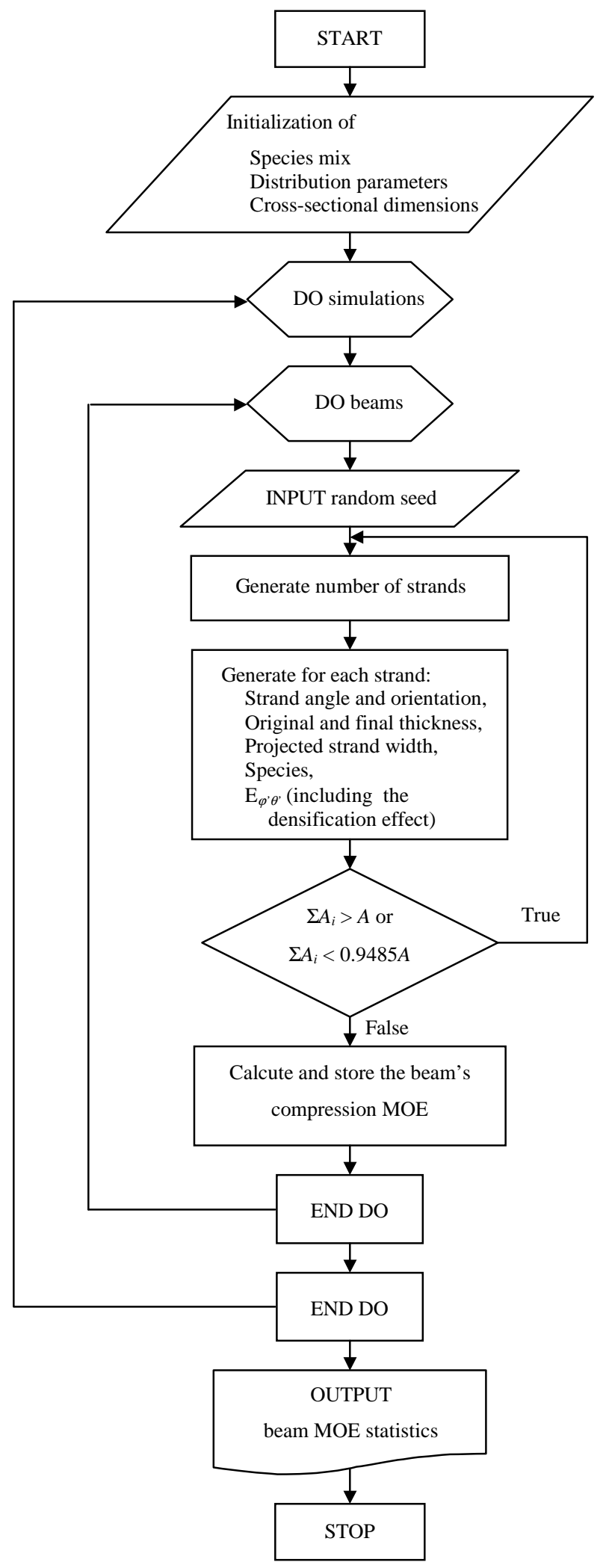

Figure E.4 - Flowchart of the program P SLComp 


\section{(Program P SLComp continued)}

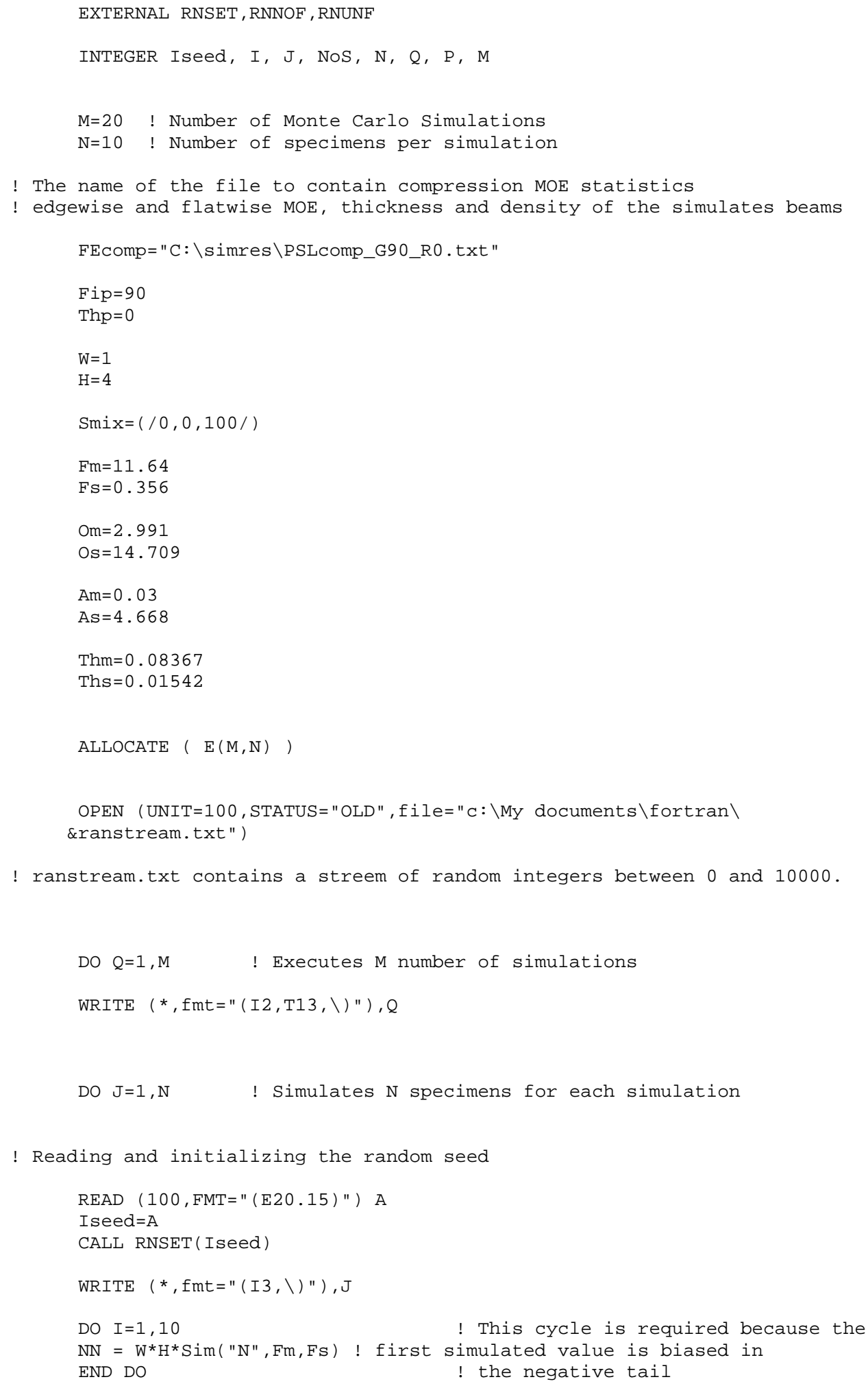




\section{(Program P SLComp continued)}

! Simulation of the strand number

1

$\mathrm{NN}=\mathrm{W}^{*} \mathrm{H}^{\star} \mathrm{Sim}(\mathrm{N} ", \mathrm{Fm}, \mathrm{FS})$ ! Simulates the number of strands

NOS $=$ INT (NN) ! Integer part (full width strands)

$\mathrm{LO}=\mathrm{NN}-\mathrm{NoS} \quad$ ! Fractional part (width of strand nr 0. )

! Simulation of strand parameters

DO $I=0$, NOS

$\operatorname{Ori}(I)=\operatorname{Sim}(" N "$, Om, Os $)$

Ang $(I)=\operatorname{Sim}(" N ", A m, A s)$

$\operatorname{Sps}(I)=$ SChoose $($ SMIX)

$\mathrm{THO}(\mathrm{I})=\operatorname{Orig}(\operatorname{Sps}(\mathrm{I}))$

$\operatorname{Lnt}(I)=1 / \operatorname{SQRT}(\operatorname{COSD}(\operatorname{Ang}(I)) * * 2 * \operatorname{COSD}(\operatorname{Ori}(\mathrm{I})) * * 2+\operatorname{SIND}(\operatorname{Ori}(\mathrm{I})) * * 2)$

Tho $(I)=\operatorname{Orig}(\operatorname{Sps}(I))$

$5 \quad \operatorname{Thi}(\mathrm{I})=\operatorname{Sim}(" \mathrm{~N} "$, Thm, Ths $)$

$\operatorname{Treal}=\operatorname{THI}(I) * \operatorname{SQRT}(\operatorname{SIND}(\operatorname{Ori}(I)) * * 2 * \operatorname{COSD}(\operatorname{Ang}(I)) * * 2+\operatorname{CoSD}(\operatorname{Ori}(I)) * * 2)$

IF (Treal>Tho(I)) THEN ! This makes sure that the final thickness is GO TO 5 ! Smaller than the original.

END IF

Fii=Fi (Ang (I), Ori (I), Fip, Thp)

The=Theta (Ang (I), Ori (I), Fip, Thp)

MOE (I) $=$ CompHank (Fii, The, Sps (I)) *Dens (Tho (I), Treal, Sps (I))

END DO

$\operatorname{Lnt}(0)=\operatorname{Lnt}(0) * \operatorname{L} 0$

! Specimen rejections based on the combined cross-section

If $\left(\operatorname{SUM}\left(\operatorname{Thi}(0: \operatorname{NoS}){ }^{*} \operatorname{Lnt}(0: \operatorname{NoS})\right) /\left(\mathrm{W}^{\star} \mathrm{H}\right)>1\right)$ THEN

go to 1

Else If $\left(\operatorname{SUM}(\operatorname{Thi}(0: \operatorname{NoS}) * \operatorname{Lnt}(0: \operatorname{NoS})) /\left(\mathrm{W}^{*} \mathrm{H}\right)<0.95\right)$ THEN

go to 1

End If

! Calculating and storing the compression MOE of the specimen

$E(Q, J)=(\operatorname{SUM}(\operatorname{Moe}(0: \operatorname{NoS}) * \operatorname{Thi}(0: \operatorname{NoS}) * \operatorname{Lnt}(0: \operatorname{NoS}))) /\left(W^{\star} H\right)$

END DO ! Ends ' $\mathrm{N}$ '

PRINT *, " "

END DO ! Ends 'NM'

CLOSE (100)

PRINT *, " "

! Saving the summary statistics of every simulation run; 


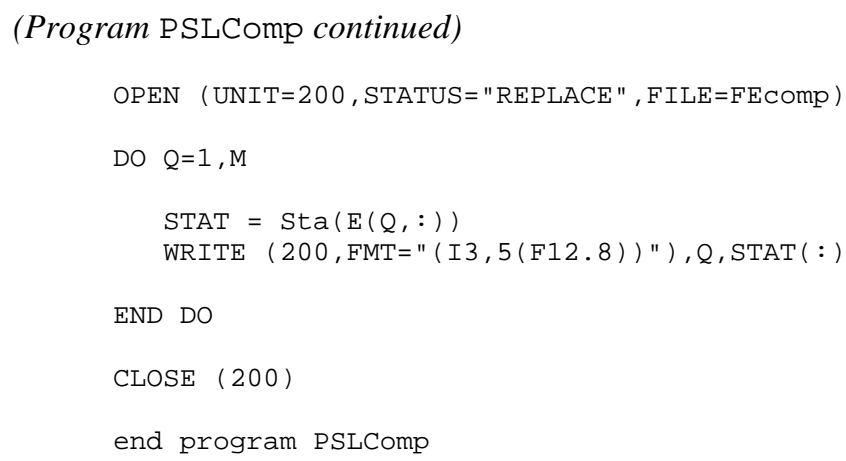

\section{E.2 Modules}

\section{The Species module}

The Species module contains species-specific information concerning the mechanical, physiscal and geometric properties, and the densification curves of the different species. The information is organized into subroutines, which are called by another subroutine (Spp), which, in turn, is called by the individual functions that need to access species-specific information. The Spp subroutine can be called from any subroutine which uses the Species module, and has only one argument; species code.

The Species module can be expanded to include further species. In this case, further subroutines should be added that initialize the same variables as the subroutines Aspen, Oak and Yelpop. Table E.2 shows the interpretation of each variable. If new species are added, the case construct in the subroutine Spp should include new cases with new species codes, which call the appropriate subroutines. 
Table E.2 - The species-specific parameters specified in the Species module

\begin{tabular}{|c|c|}
\hline Name & Description \\
\hline $\mathrm{b} 1$ & Linear coefficient of the densification curve \\
\hline $\mathrm{b} 2$ & Quadratic coefficient of the densification curve \\
\hline cLLm & Mean compression MOE in the longitudinal direction \\
\hline CLLs & STD of compression MOE in the longitudinal direction \\
\hline CLRm & Mean compression MOE at $\varphi=45^{\circ} ; \theta=90^{\circ}$ \\
\hline CLRs & STD of compression MOE at $\varphi=45^{\circ} ; \theta=90^{\circ}$ \\
\hline cLTm & Mean compression MOE at $\varphi=45^{\circ} ; \theta=0^{\circ}$ \\
\hline CLTs & STD of compression MOE at $\varphi=45^{\circ} ; \theta=0^{\circ}$ \\
\hline $\mathrm{CRRm}$ & Mean compression MOE in the radial direction \\
\hline CRRs & STD of compression MOE in the radial direction \\
\hline $\mathrm{cRTm}$ & Mean compression MOE at $\varphi=90^{\circ} ; \theta=45^{\circ}$ \\
\hline cRTs & STD of compression MOE at $\varphi=90^{\circ} ; \theta=45^{\circ}$ \\
\hline CTTm & Mean compression MOE in the tangential direction \\
\hline cTTs & STD of compression MOE in the tangential direction \\
\hline dLLm & Mean dynamic MOE in the longitudinal direction \\
\hline dLLs & STD of dynamic MOE in the longitudinal direction \\
\hline dLTm & Mean dynamic MOE in the longitudinal direction \\
\hline dLTs & STD of dynamic MOE in the longitudinal direction \\
\hline $\mathrm{Dm}$ & Average veneer density \\
\hline Ds & STD of veneer density \\
\hline dTTm & Mean dynamic MOE in the tangential direction \\
\hline dTTs & STD of dynamic MOE in the tangential direction \\
\hline $\mathrm{Td}^{*}$ & Original veneer distribution function ; type \\
\hline $\mathrm{Tm}$ & Original veneer distribution function ; location parameter \\
\hline Ts & Original veneer distribution function ; scale parameter \\
\hline $\operatorname{Tsh} 1$ & Original veneer distribution function ; shape parameter 1 \\
\hline Tsh2 & Original veneer distribution function ; shape parameter 2 \\
\hline $\begin{array}{l}\times 2 \\
\times 3 \\
\times 4\end{array}$ & $\begin{array}{l}\text { Coefficients of } x^{2}, x^{3} \text {, and } x^{4} \text { when calculating the variance at a given } \\
\text { densification level in the densification curve (see discussion at function } \\
\text { Dens.) }\end{array}$ \\
\hline Vdens & Variance of the $\Delta E$ values calculated around the densification curve \\
\hline
\end{tabular}

"Type Character. All other values are type Double Precision. 


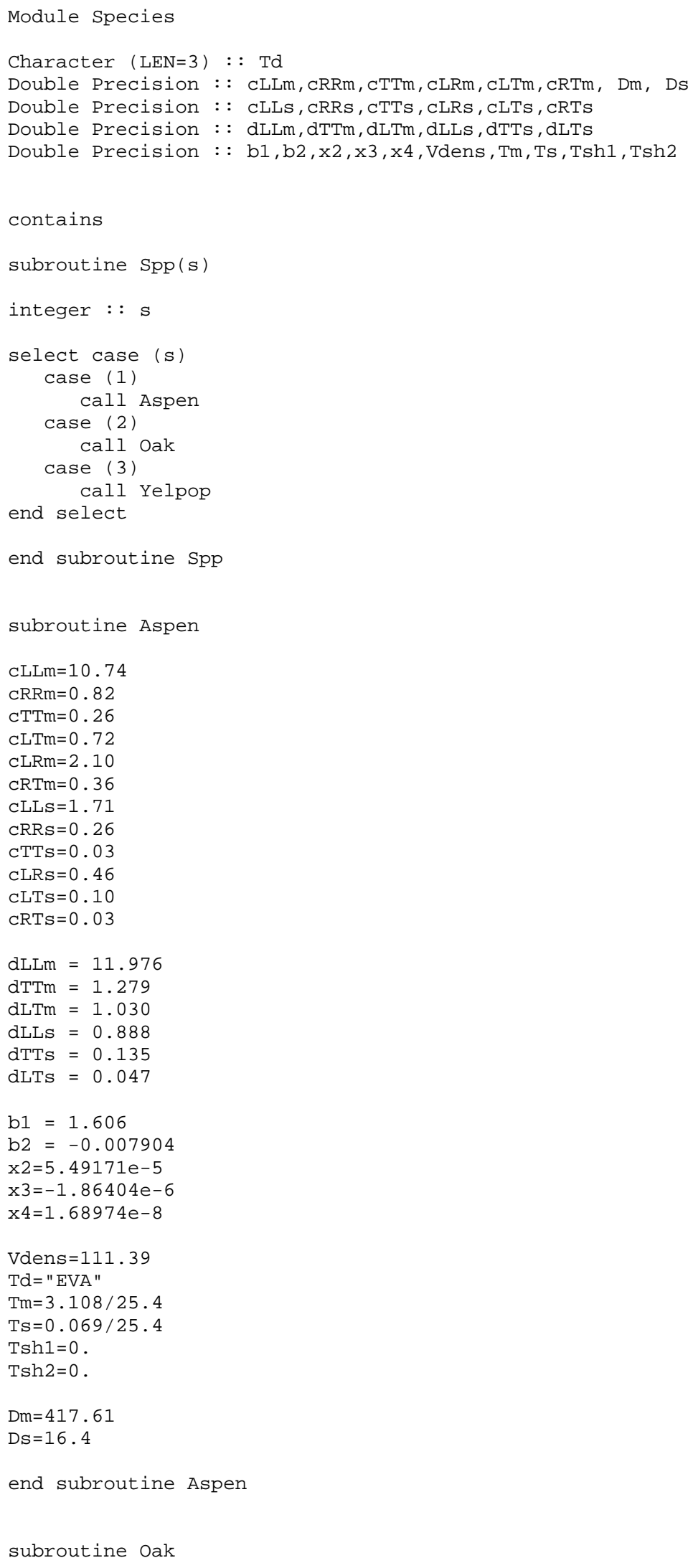


(Module Species continued)

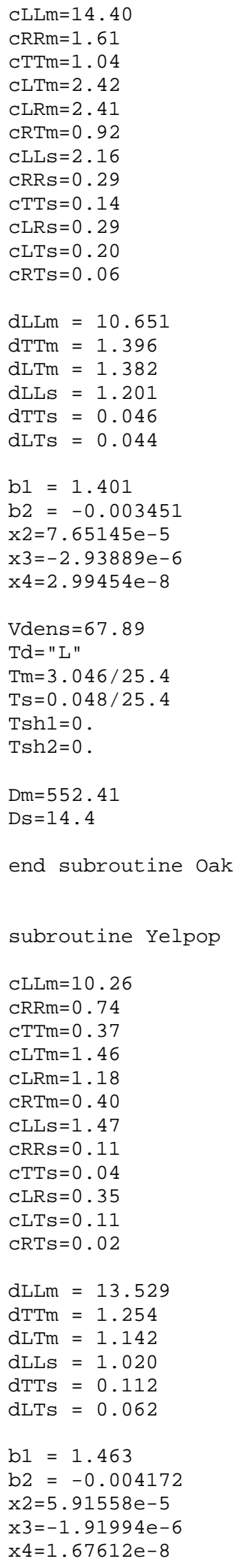


(Module Species continued)

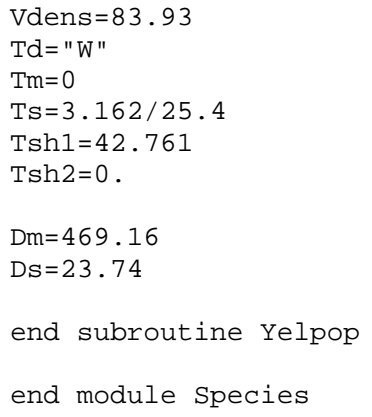

The Ifaces module

The Ifaces module contains interfaces to the functions that require one. It should be used by all programs and functions that call such functions. Since this module is easily reproducible from the declaration part of these functions, source code is not given for this module.

\section{E.3 Functions}

The following functions are called by some or all of the simulation routines and, sometimes, by other functions. The arguments of the functions are double precision floating numbers, unless otherwise noted. An exception is the species code argument, which must be an integer number. Function results are double precision numbers or arrays. Angles are always in degrees. 


\section{The CompHank and Comport functions}

Syntax: CompHank ( grain angle, ring angle, species code)

Comport ( grain angle, ring angle, species code)

Description: The CompHank and CompOrt functions simulate the compression MOE of wood at given grain and ring angle levels, according to the three-dimensional Hankinson formula and the orthotropic tensor theory, respectively. First, the functions call the SPP subroutine that initializes the parameters of the species according to the species code argument. Following this, they simulate the input parameters (MOE values) for the equation. Finally, they calculate the MOE value from the simulated parameters and the ring angle and grain angle arguments. This MOE is returned as a result, unless it is less than 0.05 , in which case the result is set to $0.05 \mathrm{GPa}$.

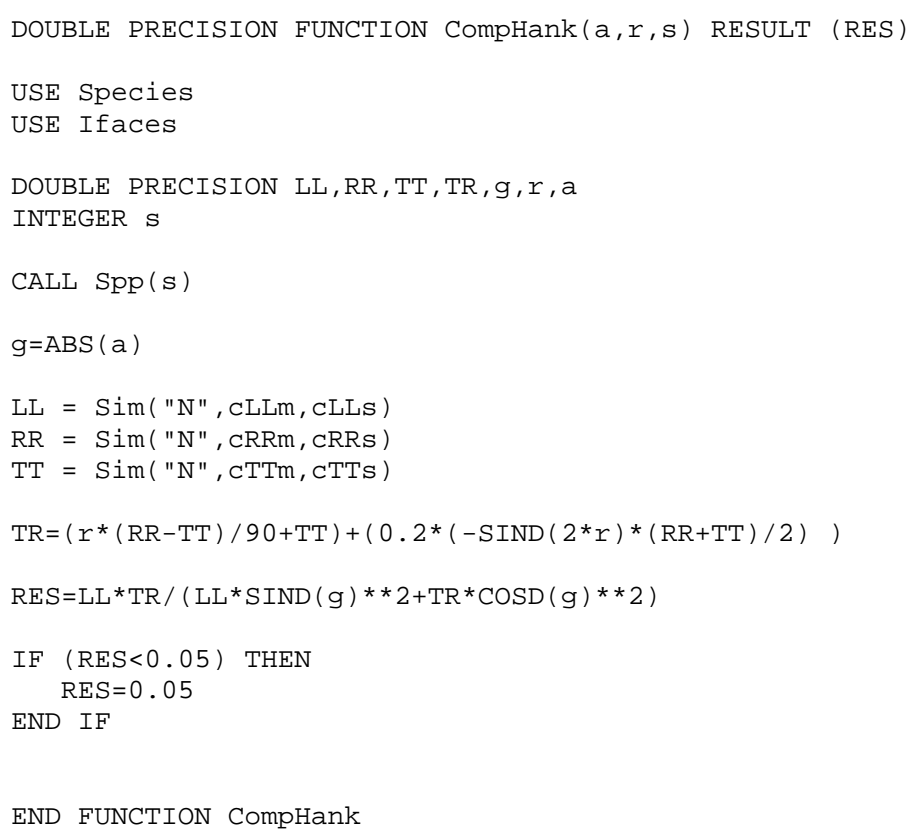




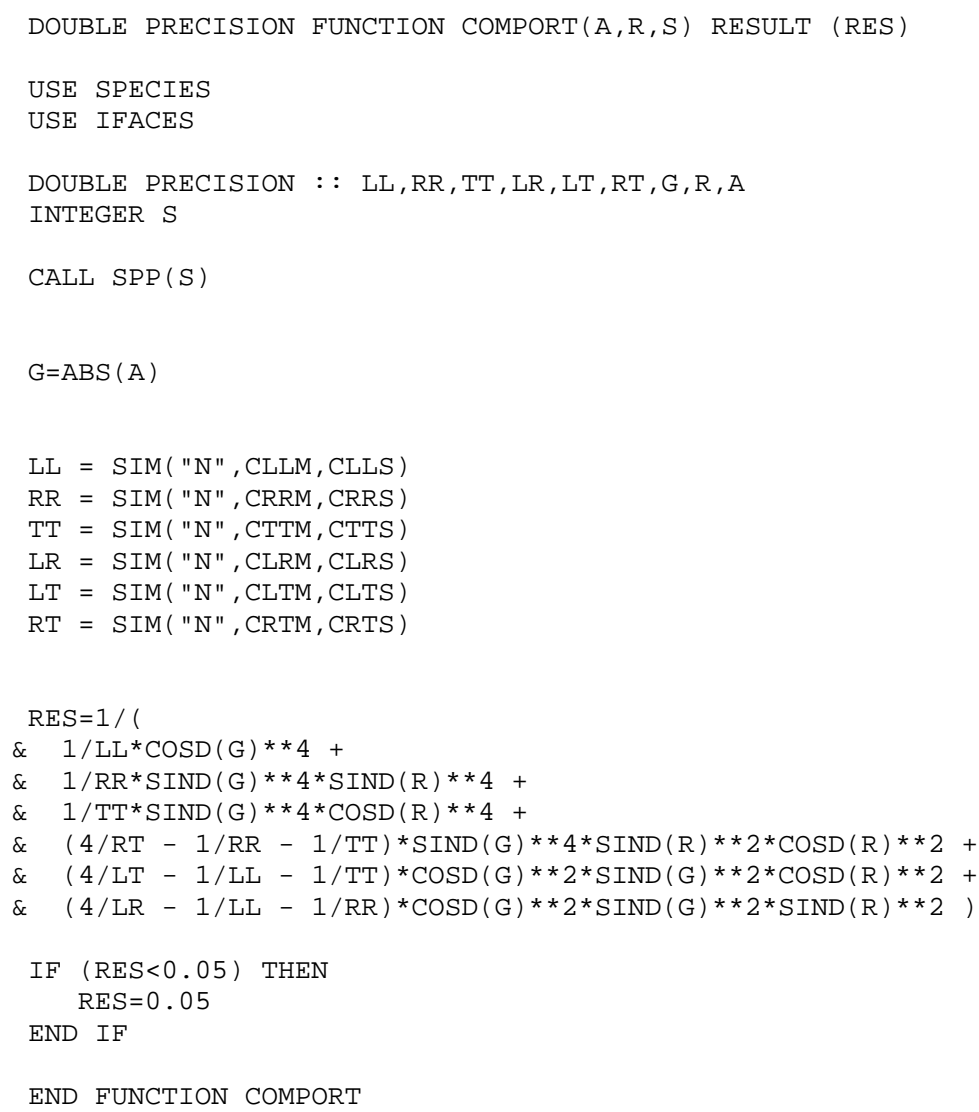

The Core function

Syntax: $\quad$ Core ( original thickness)

Description: The Core function simulates the core layer thickness in LVL If the simulated thickness is less than 0.07in, it is set to this value, and if it is higher than the original thickness, it is re-simulated. 


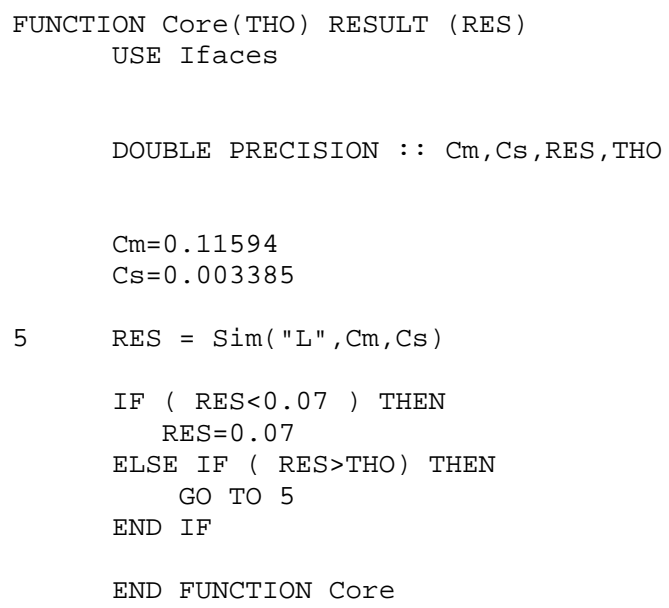

$\underline{\text { The Dens function }}$

Syntax: Dens ( original thickness, final thickness, species)

Description: The Dens function simulates a factor by which to multiply the generated MOE values to account for their increase due to the densification sustained by the constituent during pressing. The function first calls the SPP subroutine to initialize the parameters for the specified species, then calculates the percent densification from the arguments original thickness and final thickness. Following this, it calculates the value of the second order densification function (Figure 6.14), and the standard deviation at the given densification level. (The latter calculation is explained following the code.) Finally, it simulates the MOE increase, using the function value and the standard deviation as the location and scale parameters in a normal distribution. This number is divided by 1.24 (see section 6.2.2.) The calculated factor is limited in the range of 0.7 to 2.5 . If the final thickness is larger than the original one (i.e. negative densification), the function does not detect the error, but the returned densification value will be meaningless. 


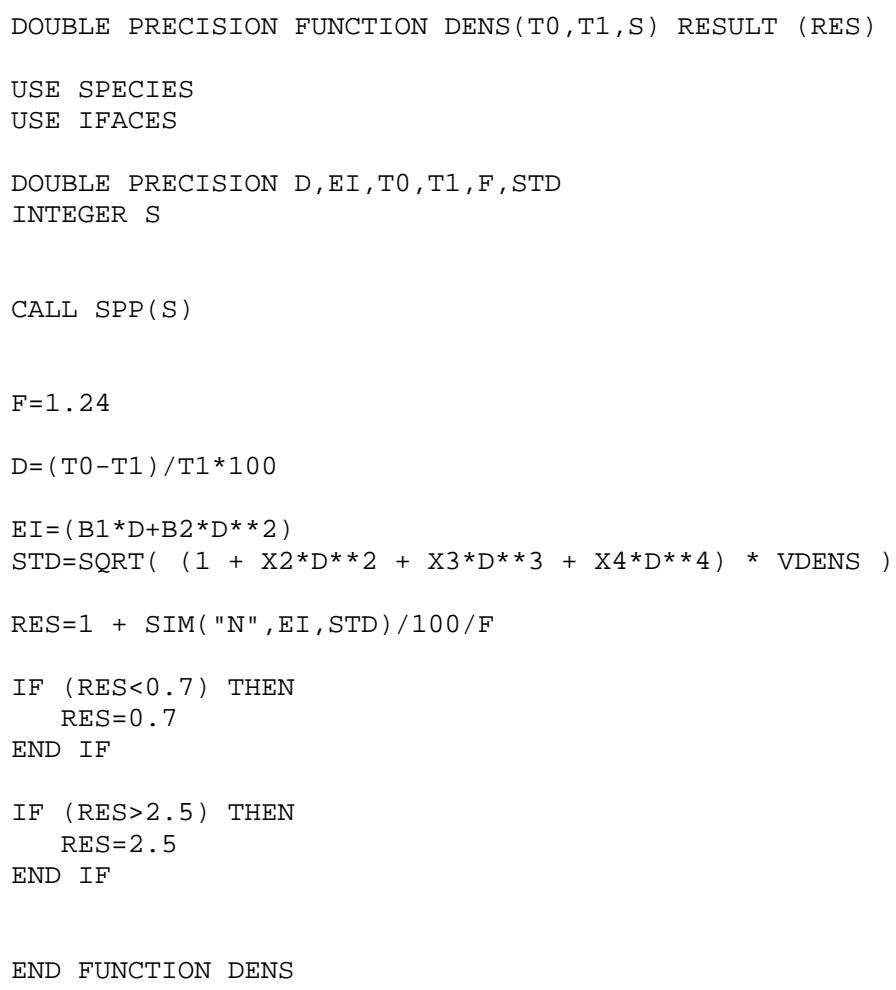

END FUNCTION DENS 


\section{Calculating THE VARIANCE OF THE DATA AT A POINT FOR SECOND-ORDER POLYNOMIAL REGRESSION}

Polynomial regression can be treated as a multiple linear regression model. E.g. for a second order line, the following model applies:

$Y_{i}=\beta_{0}+\beta_{1} X_{1 i}+\beta_{2} X_{2 i}+\varepsilon_{i}$

where $X_{1 i}=x_{i}$ and $X_{2 i}=x_{i}^{2}$ and $\varepsilon_{i} \sim \operatorname{NID}\left(\mathrm{o}, \sigma^{2}\right)$ The following calculation provides the variance at a given value of $x$ :

$V=\left(1+\left[X_{0}\right]\left[X^{\prime} X\right]^{-1}\left[X_{0}\right]^{\prime}\right) \sigma^{2}$

where $X_{0}$ is the vector $\left[1, x, x^{2}\right]$, and $\left[X^{\prime} X\right]^{-1}$ is the inverse of the following matrix:

$\left[X^{\prime} X\right]=\left[\begin{array}{ccc}n & \Sigma X_{1 i} & \Sigma X_{2 i} \\ \Sigma X_{1 i} & \Sigma X_{1 i}{ }^{2} & \Sigma X_{1 i} X_{2 i} \\ \Sigma X_{2 i} & \Sigma X_{1 i} X_{2 i} & \Sigma X_{2 i}{ }^{2}\end{array}\right]=\left[\begin{array}{ccc}n & \Sigma x_{i} & \Sigma x_{i}{ }^{2} \\ \Sigma x_{i} & \Sigma x_{i}{ }^{2} & \Sigma x_{i}{ }^{3} \\ \Sigma x_{i}{ }^{2} & \Sigma x_{i}{ }^{3} & \Sigma x_{i}{ }^{4}\end{array}\right]$

where $x_{i}$ are the $x$ values belonging to the individual measurements, and $n$ is the total number of measurements.

In case of a regression line that passes through the origin $\left(b_{0}=0\right)$, the first row and column of the matrix, as well as the first element of $X_{0}$, are eliminated.

The Dty function

Syntax: Dty (species)

Description: The Dty function simulates the original density of a layer or a strand. First, it calls the SPP subroutine to initialize the parameters for the given species, than it simulates the species-specific density using these parameters. This function contains no safety limits on the simulated density, since its value is not crucial for the simulation of the mechanical properties. 


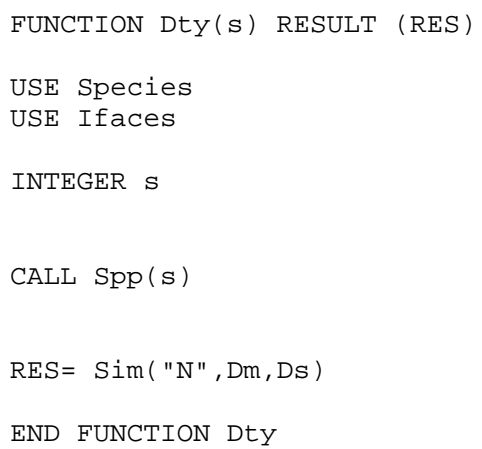

The Face function

\section{Syntax: $\quad$ Face (original thickness)}

Description: The Face function simulates the face layer thickness in LVL If the simulated thickness is less than 0.07in, it is set to this value, and if it is higher than the original thickness, it is re-simulated.

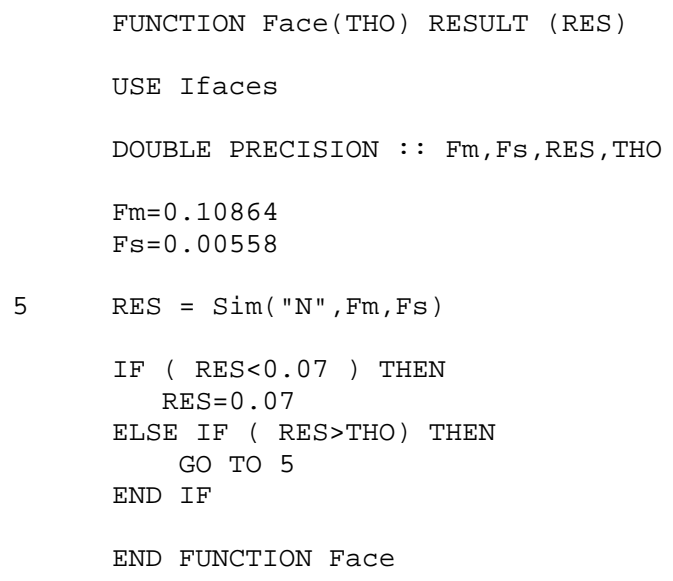




\section{The Fi function}

Syntax: $\quad \operatorname{Fi}\left(\alpha, \beta, \varphi^{\prime}, \theta^{\prime}\right)$

Description: The Fi function calculates the grain and ring angle between a given strand and the load applied on PSL. The first two arguments are the angle and deviation of the given strand (see section 5.4.3), while the second two are the load and strand orientation of the applied load. Note that in bending, $\varphi^{\prime}=0^{\circ}$, and that $\beta$ is not required for the calculation. This parameter is only included here for the sake of consistency with the function Theta. For the derivation of this calculation, see APPENDIX C.

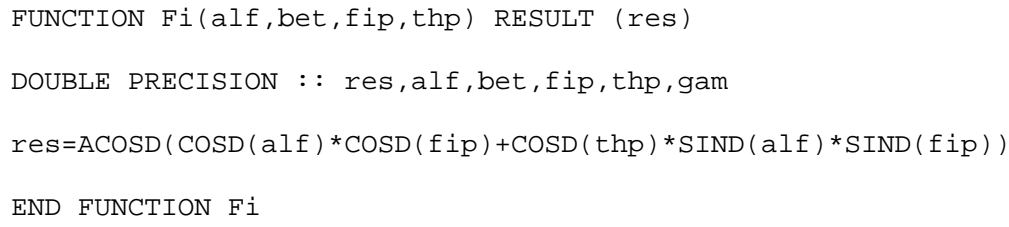

The Numint function

Syntax: Numint ( orientation, position, thickness, length, height, resolution)

Description: The Numint function uses numerical integration to calculate $I_{T}$ and $I_{C}$ for a single strand cross-section in PSL. The arguments are the following:

orientation - the cross-sectional orientation of the strand, relative to the neutral axis;

position - the position of the strand in the loading direction;

thickness - projected strand thickness;

length - projected length of the strand in the cross-section;

height - the cross-sectional dimension of the beam in the loading direction;

resolution - the resolution of the numerical integration.

The function first defines a number of parameters; Figure E.5 aids the understanding of their meaning. After this, the function divides the strand crosssection into resolution number of segments, also shown on Figure E.5. The segments are treated as rectangles, and their $2^{\text {nd }}$ order moment of inertia is 
calculated respective to the neutral axis of the beam cross-section. Depending on whether the midpoints of the intervals are in the tension or compression region, their moment of inertia is added to $I_{T i}$ or $I_{C i}$, respectively. If the segment is outside of the beam cross-section, the function repositions it according to the torus convention. After the moment of inertia for all of the segments have been calculated and added to the appropriate $I$ value, the function returns an array containing $I_{T i}$ and $I_{C i}$ in this order.

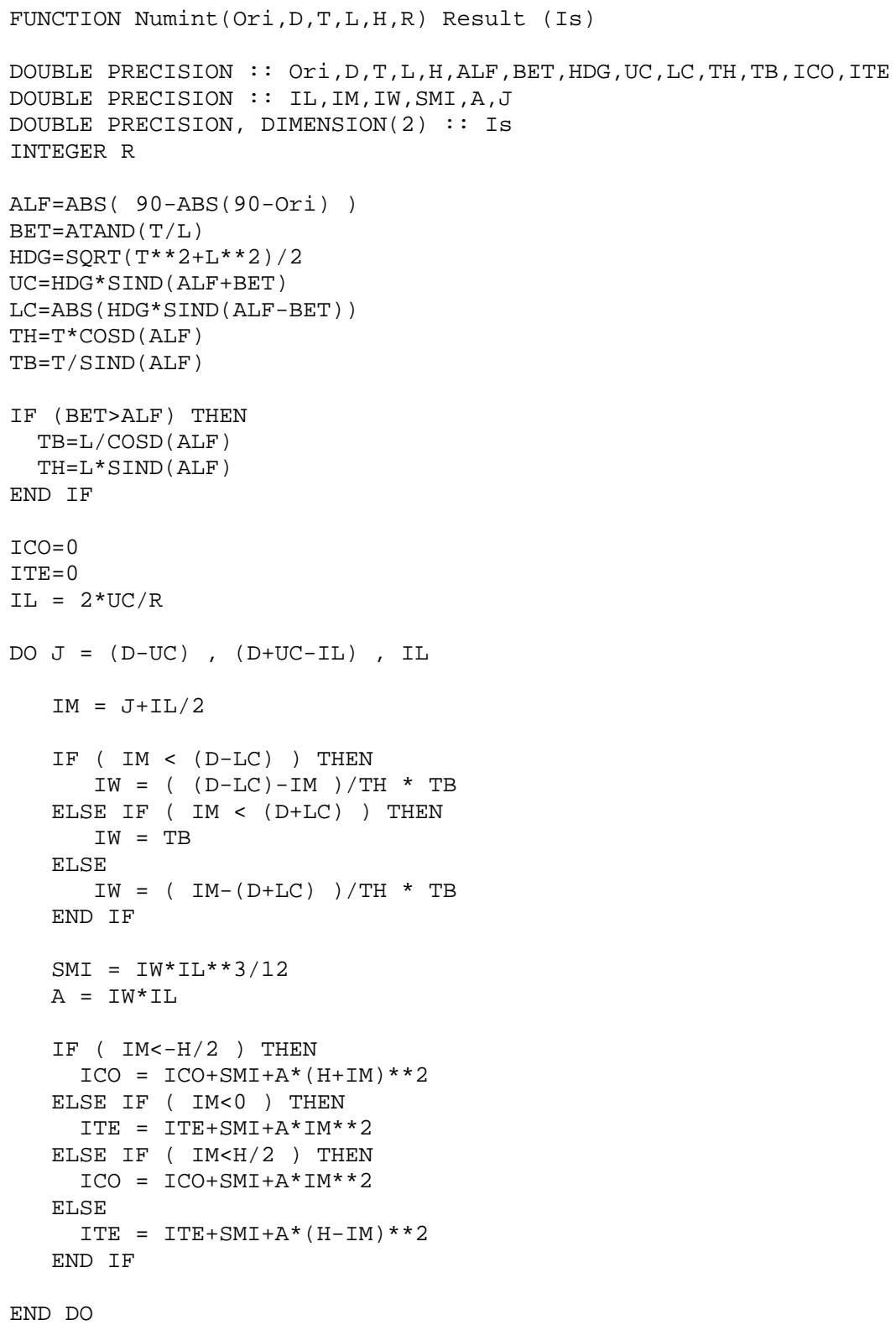



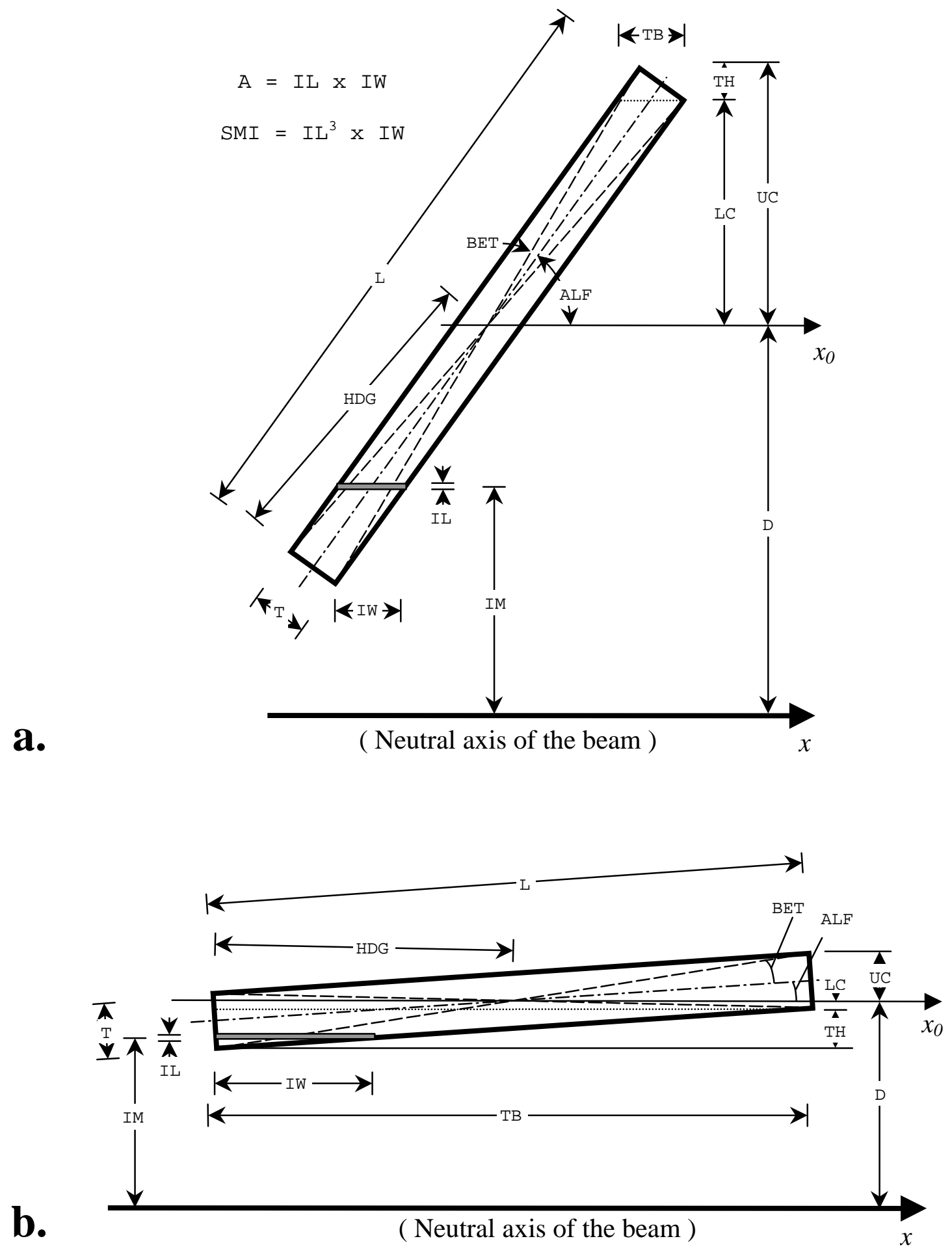

Figure E.5 - Variables used in the function Numint
a. - When ALF $>$ BET
b. - When ALF $<B E T$ 
(Function Numint continued)

Is $(1)=$ ICO
Is $(2)=$ ITE

END FUNCTION Numint

The Orig function

Syntax: $\quad$ Orig(

Description: The Orig function simulates the original layer or strand thickness for a constituent. First, it calls the SPP subroutine to initialize the parameters for the given species, than it simulates the species-specific original thickness, using these parameters. If the minimum specified thickness is $0.11 \mathrm{in}$, because, below this value it gets hard to simulate LVL core layer thickness values, which must be smaller than the original thickness value.

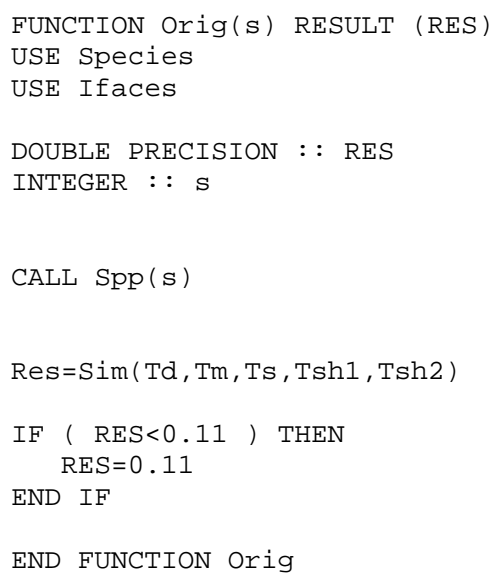

The Reg function

Syntax: $\quad \operatorname{Reg}(\mathrm{x}$ values, y values )

Description: The Reg function takes a two one-dimensional, arbitrary sized arrays as its arguments. The corresponding values in the two arrays are treated as data pairs. The function returns an array of three values, containing the linear regression parameters $b 0$ ( $y$-intercept), $b 1$ (slope), and $\mathrm{r}^{2}$, in this order. 


\section{Warnings: Requires interface!}

The size of the two arrays must be the same.

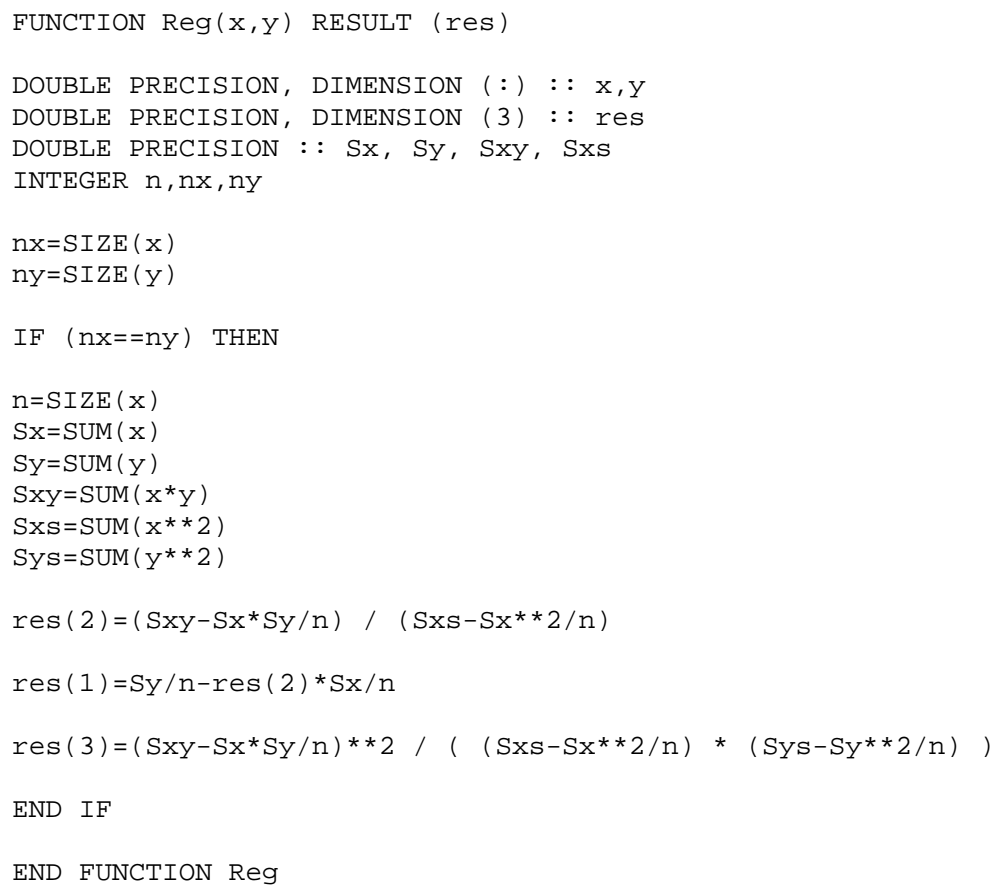

The SChoose function

Syntax: $\quad$ SChoose ( species mix)

Description: The purpose of the SChoose function is to randomly generate a PSL strand from a given species mix. The argument species mix is a one-dimensional array that contains the proportions of the species coded 1 to $n$, where $n$ is the total number of species. Note that the array can be any size, so that the mix may contain any number of species (this feature is useful if the raw data base is to be expanded by adding new species in the module Species.) The proportions do not have to add up to any particular value (e.g. 1 or 100$)$

\section{Warning: $\quad \underline{\text { Requires interface }}$}




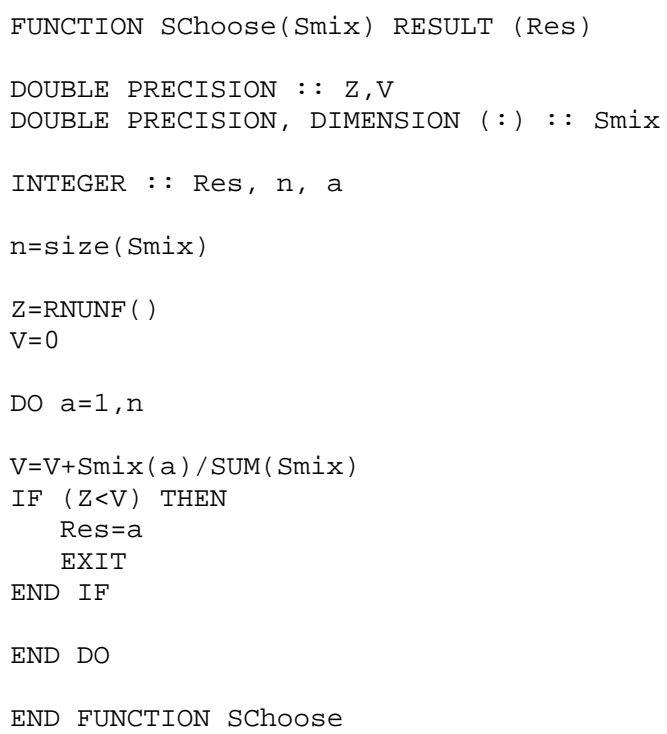

The Sim function

Syntax: Sim(distribution, location, scale, shape1, shape2)

Description: The Sim function simulates a random number from a given statistical distribution, specified in the distribution argument. This is a character type argument which can assume the following values:
$\mathrm{N} \quad$ - normal distribution;
U - uniform distribution;
L - logistic distribution;
W - Weibull distribution;
JSU - Johnson SU distribution;
EVA - extreme value, type A distribution;
EVB - extreme value, type B distribution;

The location and scale arguments are mandatory, while shape 1 and shape 2 are optional, since they are not necessary in every distribution function.

The source code is self-explanatory.

Warning: Requires interface! 


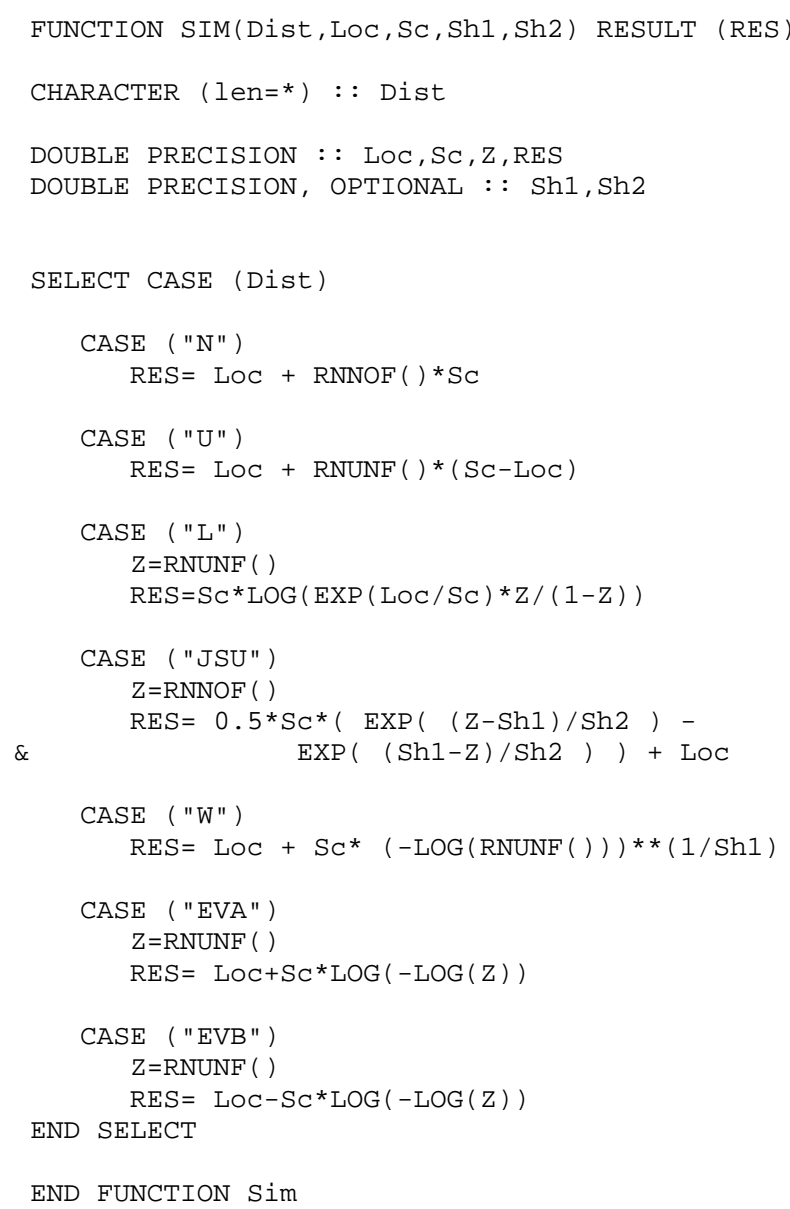

The St a function

Syntax: $\quad$ Sta ( array)

Description: The Sta function takes a one-dimensional, arbitrary sized array as its argument, and returns an array of five values, containing the mean, standard deviation, minimum, maximum and skewness of the data contained in array, in this order.

Warning: Requires interface! 


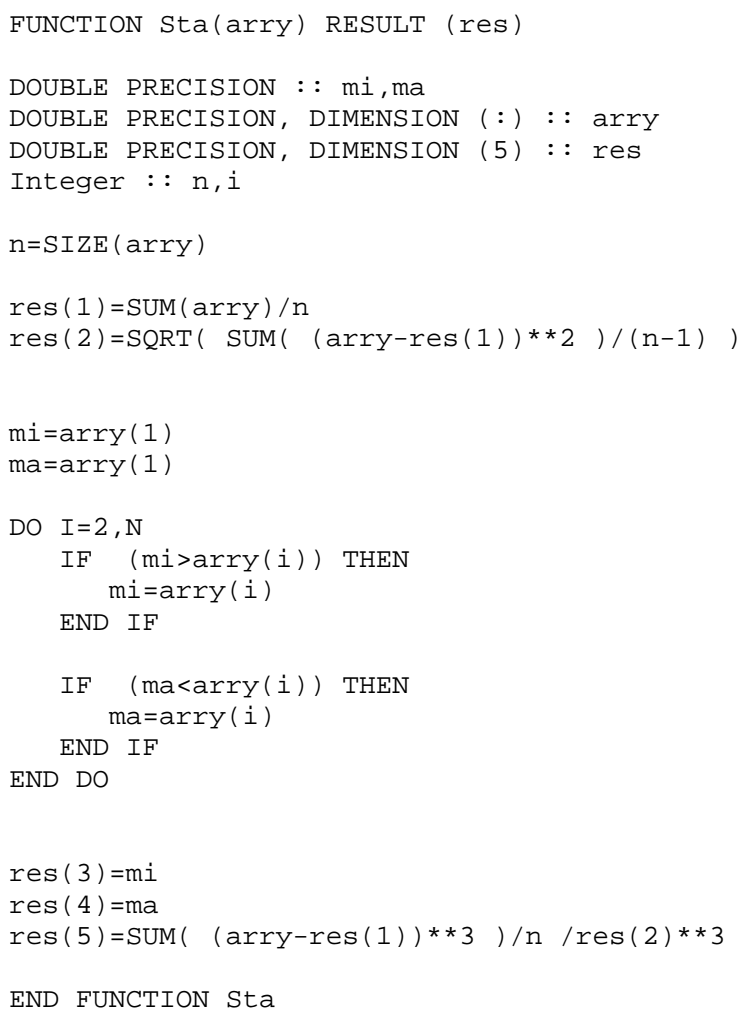

The TenMOE function

Syntax: TenMOE (grain angle, species code)

Description: The TenMOE function simulates the tensile MOE of different wood species at a given grain angle level, using the combination of the orthotropic theory and Hankinson's formula. First, the function calls the SPP subroutine that initializes the parameters of the species according to the species code argument. Following this, it simulates the input parameters (dynamic MOE values) for the model. After this, the dynamic MOE value is calculated using both the orthotropic equation and Hankinson's formula, and the two values are combined using Equation 6.3. The simulated dynamic MOE is converted to tensile MOE. For this, the program calculates the static MOE belonging to the simulated dynamic MOE, according to the second order relationship shown on Figure 6.13 b, and calculates the standard deviation at the given dynamic MOE level. (For a discussion of this calculation, 
see the Dens function) Finally, the function simulates the static MOE from the calculated mean static MOE and the standard deviation. This value is returned as a result, unless it is less than 0.05 , in which case the result is set to $0.05 \mathrm{GPa}$.

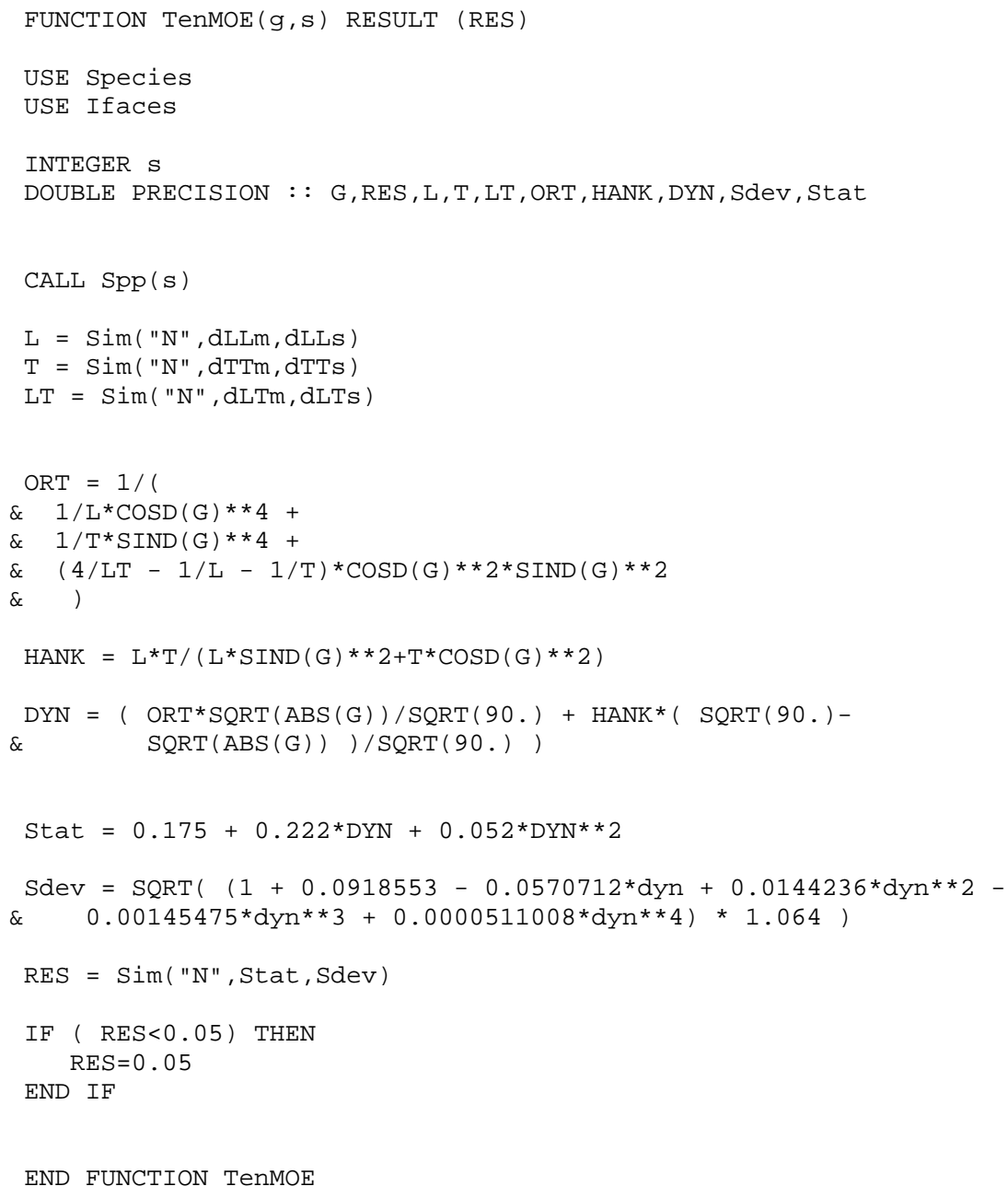




\section{The Thet a function}

Syntax: $\quad$ Theta $\left(\alpha, \beta, \varphi^{\prime}, \theta^{\prime}\right)$

Description: The Thet a function, as a counterpart of the $\mathrm{F} i$ function calculates the ring angle between a given strand and the load applied on PSL. For a description of the arguments, see $\mathrm{Fi}$. Note that this function can be used to calculate $\theta$ for LVL layers, too, which can be regarded as PSL strands with $\alpha=0^{\circ}$ and $\beta=0^{\circ}$. (The Fi function can also be used in this way, but it will simply return $\varphi^{\prime}$ as a result.) For the derivation of this calculation, see APPENDIX $C$.

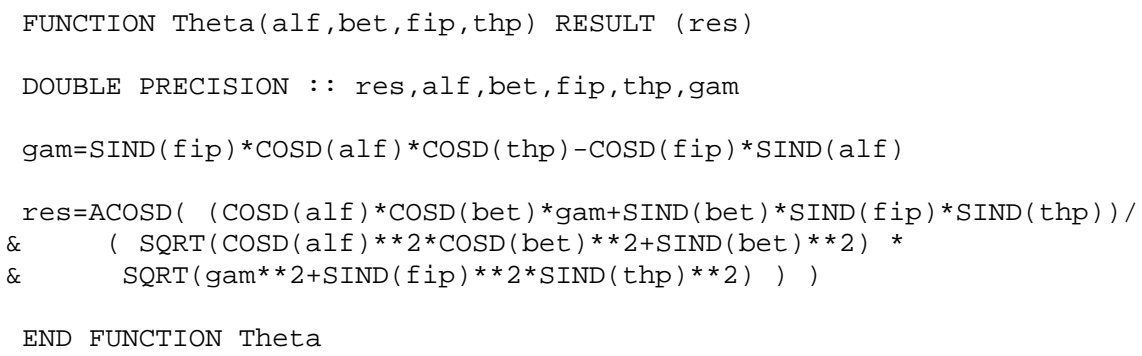




\section{APPENDIX F - RESULTS OF THE SIMULATION RUNS}

\section{F.1 The properties of $L V L$}

Table F.1 - LVL thickness (mm)

\begin{tabular}{lccccc}
\hline \hline Sim. & Mean & STD & Min. & Max & Skewness \\
\hline 1 & 43.05 & 0.44 & 42.34 & 43.93 & 0.528 \\
2 & 42.81 & 0.55 & 41.88 & 43.90 & 0.238 \\
3 & 42.76 & 0.47 & 42.18 & 43.95 & 0.922 \\
4 & 42.86 & 0.61 & 41.96 & 44.01 & 0.507 \\
5 & 42.90 & 0.46 & 42.16 & 43.85 & 0.347 \\
6 & 43.04 & 0.34 & 42.26 & 43.49 & -0.882 \\
7 & 43.02 & 0.59 & 42.14 & 44.05 & 0.356 \\
8 & 42.97 & 0.43 & 42.16 & 43.61 & -0.159 \\
9 & 42.84 & 0.55 & 41.83 & 43.60 & -0.244 \\
10 & 42.97 & 0.54 & 42.06 & 44.07 & 0.293 \\
11 & 43.01 & 0.56 & 41.79 & 44.05 & -0.534 \\
12 & 43.05 & 0.52 & 42.20 & 44.35 & 0.625 \\
13 & 43.16 & 0.43 & 42.40 & 43.96 & 0.134 \\
14 & 42.94 & 0.36 & 42.12 & 43.46 & -0.477 \\
15 & 43.31 & 0.54 & 42.30 & 44.01 & -0.145 \\
16 & 42.85 & 0.59 & 41.61 & 43.70 & -0.661 \\
17 & 42.70 & 0.49 & 41.52 & 43.49 & -0.472 \\
18 & 43.03 & 0.53 & 42.10 & 44.00 & 0.097 \\
\hline 19 & 43.05 & 0.47 & 42.13 & 43.92 & 0.009 \\
20 & 42.94 & 0.49 & 41.91 & 43.66 & -0.390 \\
\hline Avg. & $\mathbf{4 2 . 9 6}$ & $\mathbf{0 . 5 0}$ & $\mathbf{4 2 . 0 5}$ & $\mathbf{4 3 . 8 5}$ & $\mathbf{0 . 0 0 5}$ \\
\hline Exp. & $\mathbf{4 3 . 5 6}$ & $\mathbf{0 . 1 5}$ & $\mathbf{4 3 . 1 3}$ & $\mathbf{4 3 . 9 7}$ & $\mathbf{0 . 0 1 3}$ \\
\hline \hline
\end{tabular}


Table F.2 - LVL density $\left(\mathrm{kg} / \mathrm{m}^{3}\right)$

\begin{tabular}{lrrrrr}
\hline \hline Sim. & Mean & STD & Min. & Max & Skew. \\
\hline 1 & 511 & 10 & 492 & 533 & 0.145 \\
2 & 513 & 11 & 494 & 534 & -0.065 \\
3 & 514 & 8 & 493 & 525 & -1.148 \\
4 & 512 & 12 & 486 & 533 & -0.073 \\
\hline 5 & 511 & 9 & 488 & 524 & -0.696 \\
6 & 512 & 8 & 496 & 528 & -0.143 \\
7 & 512 & 9 & 496 & 526 & -0.144 \\
8 & 512 & 11 & 492 & 537 & 0.260 \\
9 & 510 & 11 & 490 & 527 & -0.207 \\
10 & 513 & 11 & 496 & 539 & 0.479 \\
11 & 508 & 12 & 476 & 533 & -0.356 \\
12 & 510 & 6 & 497 & 519 & -0.576 \\
13 & 511 & 14 & 492 & 536 & 0.431 \\
14 & 511 & 8 & 498 & 525 & 0.145 \\
15 & 508 & 10 & 488 & 528 & 0.222 \\
16 & 513 & 9 & 496 & 531 & 0.014 \\
17 & 517 & 9 & 488 & 529 & -1.336 \\
18 & 510 & 8 & 500 & 527 & 0.500 \\
19 & 512 & 10 & 490 & 533 & -0.250 \\
20 & 508 & 11 & 493 & 533 & 0.799 \\
\hline Avg. & $\mathbf{5 1 1}$ & $\mathbf{1 0}$ & $\mathbf{4 9 2}$ & $\mathbf{5 3 0}$ & $\mathbf{- 0 . 1 0 0}$ \\
\hline Exp. & $\mathbf{5 6 6}$ & $\mathbf{1 1}$ & $\mathbf{5 4 1}$ & $\mathbf{5 8 4}$ & $\mathbf{0 . 0 7 8}$ \\
\hline \hline
\end{tabular}

Table F.3 - LVL Bending MOE - edgewise (GPa)

\begin{tabular}{lrrrrr}
\hline \hline Sim. & Mean & STD & Min. & Max & Skew. \\
\hline 1 & 12.76 & 0.32 & 12.33 & 13.67 & 1.214 \\
2 & 12.89 & 0.40 & 11.97 & 13.76 & -0.519 \\
\hline 3 & 12.77 & 0.50 & 11.80 & 13.57 & -0.336 \\
\hline 4 & 12.82 & 0.41 & 12.15 & 13.64 & 0.252 \\
\hline 5 & 12.71 & 0.42 & 11.93 & 13.29 & -0.311 \\
\hline 6 & 12.69 & 0.37 & 11.93 & 13.50 & 0.264 \\
\hline 7 & 12.66 & 0.41 & 11.94 & 13.38 & 0.011 \\
\hline 8 & 12.80 & 0.48 & 11.90 & 13.74 & 0.030 \\
\hline 9 & 12.57 & 0.37 & 11.94 & 13.24 & -0.162 \\
10 & 12.71 & 0.43 & 12.10 & 13.37 & 0.196 \\
\hline 11 & 12.62 & 0.53 & 11.72 & 13.36 & -0.293 \\
12 & 12.57 & 0.39 & 11.90 & 13.39 & 0.261 \\
\hline 13 & 12.62 & 0.52 & 11.77 & 13.65 & 0.101 \\
14 & 12.75 & 0.31 & 12.06 & 13.21 & -0.339 \\
\hline 15 & 12.55 & 0.45 & 11.56 & 13.47 & -0.254 \\
\hline 16 & 12.62 & 0.36 & 12.13 & 13.81 & 1.717 \\
\hline 17 & 12.80 & 0.44 & 11.74 & 13.71 & -0.322 \\
\hline 18 & 12.76 & 0.30 & 12.18 & 13.30 & 0.021 \\
\hline 19 & 12.66 & 0.33 & 12.14 & 13.29 & -0.118 \\
\hline 20 & 12.69 & 0.40 & 11.83 & 13.39 & -0.153 \\
\hline Avg. & $\mathbf{1 2 . 7 0}$ & $\mathbf{0 . 4 1}$ & $\mathbf{1 1 . 9 5}$ & $\mathbf{1 3 . 4 9}$ & $\mathbf{0 . 0 6 3}$ \\
\hline Exp. & $\mathbf{1 3 . 2 2}$ & $\mathbf{1 . 2 1}$ & $\mathbf{1 1 . 8 5}$ & $\mathbf{1 5 . 9 0}$ & $\mathbf{0 . 0 2 2}$ \\
\hline \hline & & & & & \\
\hline
\end{tabular}


Table F.4 - LVL Bending MOE - flatwise (GPa)

\begin{tabular}{lccccc}
\hline \hline Sim. & Mean & STD & Min. & Max & Skew. \\
\hline 1 & 12.84 & 0.73 & 11.87 & 14.44 & 0.319 \\
2 & 13.21 & 0.99 & 11.20 & 15.20 & -0.142 \\
3 & 13.17 & 0.91 & 11.23 & 15.02 & -0.237 \\
4 & 13.12 & 0.94 & 11.31 & 14.48 & -0.398 \\
\hline 5 & 13.26 & 0.63 & 12.06 & 14.77 & 0.530 \\
6 & 12.53 & 0.73 & 10.81 & 13.80 & -0.036 \\
7 & 12.90 & 0.87 & 11.43 & 14.25 & 0.046 \\
8 & 13.04 & 0.80 & 11.57 & 14.27 & -0.123 \\
9 & 13.00 & 0.75 & 11.59 & 14.47 & 0.066 \\
10 & 12.88 & 0.89 & 11.00 & 14.72 & 0.090 \\
11 & 12.81 & 0.70 & 11.88 & 13.87 & 0.058 \\
12 & 12.49 & 0.64 & 11.41 & 13.74 & 0.270 \\
13 & 12.92 & 0.86 & 11.00 & 14.76 & -0.086 \\
14 & 12.82 & 0.63 & 11.77 & 13.99 & 0.116 \\
15 & 12.79 & 0.62 & 11.68 & 13.85 & 0.158 \\
16 & 12.87 & 0.65 & 11.13 & 13.89 & -0.656 \\
\hline 17 & 12.84 & 0.96 & 11.15 & 15.09 & 0.336 \\
18 & 12.98 & 0.70 & 11.86 & 14.38 & 0.514 \\
\hline 19 & 12.73 & 1.03 & 10.58 & 14.84 & 0.260 \\
\hline 20 & 13.04 & 0.81 & 11.49 & 15.31 & 0.918 \\
\hline Avg. & $\mathbf{1 2 . 9 1}$ & $\mathbf{0 . 7 9}$ & $\mathbf{1 1 . 4 0}$ & $\mathbf{1 4 . 4 6}$ & $\mathbf{0 . 1 0 0}$ \\
\hline Exp. & $\mathbf{1 3 . 3 6}$ & $\mathbf{0 . 7 2}$ & $\mathbf{1 2 . 2 0}$ & $\mathbf{1 5 . 1 6}$ & $\mathbf{0 . 2 1 2}$ \\
\hline \hline & & & & & \\
\hline
\end{tabular}

Table F.5 - LVL Regression parameters

\begin{tabular}{lrll}
\hline \hline Sim. & \multicolumn{1}{c}{ b0 } & b1 & $\mathbf{r}^{\mathbf{2}}$ \\
\hline 1 & 8.801 & 0.309 & 0.483 \\
2 & 9.062 & 0.290 & 0.509 \\
3 & 10.531 & 0.170 & 0.097 \\
4 & 9.651 & 0.241 & 0.300 \\
\hline 5 & 7.486 & 0.394 & 0.356 \\
6 & 9.279 & 0.273 & 0.299 \\
7 & 9.276 & 0.262 & 0.307 \\
8 & 7.792 & 0.384 & 0.405 \\
\hline 9 & 9.238 & 0.257 & 0.277 \\
10 & 8.587 & 0.320 & 0.437 \\
11 & 7.413 & 0.406 & 0.287 \\
12 & 8.991 & 0.286 & 0.222 \\
13 & 7.215 & 0.419 & 0.491 \\
14 & 11.086 & 0.130 & 0.069 \\
15 & 6.994 & 0.434 & 0.353 \\
16 & 11.059 & 0.121 & 0.048 \\
\hline 17 & 9.559 & 0.253 & 0.309 \\
\hline 18 & 10.636 & 0.164 & 0.144 \\
\hline 19 & 10.453 & 0.173 & 0.300 \\
\hline 20 & 9.205 & 0.267 & 0.293 \\
\hline Avg. & $\mathbf{9 . 1 1 6}$ & $\mathbf{0 . 2 7 8}$ & $\mathbf{0 . 2 9 9}$ \\
\hline Exp. & $\mathbf{0 . 0 0 2}$ & $\mathbf{0 . 9 9 0}$ & $\mathbf{0 . 3 4 2}$ \\
\hline \hline & & & \\
\hline
\end{tabular}


Table F.6 - LVL Compression $E_{x}(\mathrm{GPa})$

\begin{tabular}{lccccc}
\hline Sim. & Mean & STD & Min. & Max & Skew. \\
\hline 1 & 11.20 & 0.37 & 10.59 & 11.67 & -0.535 \\
2 & 11.30 & 0.40 & 10.76 & 12.13 & 0.463 \\
3 & 11.40 & 0.70 & 10.00 & 12.42 & -0.326 \\
\hline 4 & 11.37 & 0.36 & 10.84 & 11.91 & -0.005 \\
\hline 5 & 11.31 & 0.35 & 10.84 & 12.03 & 0.444 \\
6 & 11.37 & 0.45 & 10.83 & 12.17 & 0.435 \\
\hline 7 & 11.54 & 0.54 & 10.86 & 12.57 & 0.452 \\
8 & 11.55 & 0.24 & 11.28 & 12.07 & 0.793 \\
\hline 9 & 11.47 & 0.41 & 10.87 & 12.02 & -0.157 \\
10 & 11.13 & 0.56 & 10.43 & 12.21 & 0.431 \\
\hline 11 & 11.37 & 0.67 & 10.52 & 12.53 & 0.210 \\
12 & 11.18 & 0.54 & 10.25 & 12.01 & -0.059 \\
\hline 13 & 11.49 & 0.43 & 10.75 & 12.17 & -0.214 \\
14 & 11.25 & 0.52 & 10.71 & 12.57 & 1.517 \\
\hline 15 & 11.28 & 0.37 & 10.57 & 11.88 & -0.330 \\
\hline 16 & 11.41 & 0.50 & 10.29 & 11.92 & -0.983 \\
\hline 17 & 11.39 & 0.36 & 10.74 & 11.97 & -0.225 \\
\hline 18 & 11.00 & 0.52 & 10.23 & 11.73 & -0.374 \\
\hline 19 & 11.37 & 0.46 & 10.65 & 12.02 & -0.076 \\
\hline 20 & 11.19 & 0.55 & 10.14 & 12.12 & -0.167 \\
\hline Avg. & $\mathbf{1 1 . 3 3}$ & $\mathbf{0 . 4 6}$ & $\mathbf{1 0 . 6 1}$ & $\mathbf{1 2 . 1 1}$ & $\mathbf{0 . 0 6 5}$ \\
\hline Exp. & $\mathbf{1 1 . 8 7}$ & $\mathbf{1 . 0 6}$ & $\mathbf{9 . 7 1}$ & $\mathbf{1 3 . 3 4}$ & $\mathbf{0 . 1 4 2}$ \\
\hline \hline & & & & & \\
\hline
\end{tabular}

Table F.7 - LVL Compression $E_{y}(\mathrm{GPa})$

\begin{tabular}{lrrrrr}
\hline \hline Sim. & Mean & STD & Min. & Max & Skew. \\
\hline 1 & 0.41 & 0.02 & 0.39 & 0.44 & 0.226 \\
2 & 0.41 & 0.01 & 0.40 & 0.43 & -0.281 \\
\hline 3 & 0.41 & 0.01 & 0.38 & 0.42 & -0.485 \\
4 & 0.42 & 0.01 & 0.40 & 0.43 & 0.173 \\
\hline 5 & 0.41 & 0.01 & 0.40 & 0.44 & 0.950 \\
6 & 0.41 & 0.01 & 0.39 & 0.43 & 0.740 \\
\hline 7 & 0.40 & 0.01 & 0.39 & 0.42 & -0.155 \\
8 & 0.41 & 0.02 & 0.39 & 0.45 & 0.503 \\
\hline 9 & 0.42 & 0.01 & 0.40 & 0.45 & 0.167 \\
10 & 0.41 & 0.02 & 0.38 & 0.44 & 0.095 \\
11 & 0.42 & 0.01 & 0.40 & 0.43 & -0.061 \\
\hline 12 & 0.41 & 0.01 & 0.39 & 0.43 & 0.490 \\
13 & 0.40 & 0.01 & 0.38 & 0.42 & 0.012 \\
14 & 0.41 & 0.01 & 0.39 & 0.43 & -0.086 \\
\hline 15 & 0.41 & 0.01 & 0.38 & 0.43 & 0.469 \\
\hline 16 & 0.40 & 0.01 & 0.38 & 0.42 & -0.590 \\
\hline 17 & 0.41 & 0.02 & 0.39 & 0.44 & 0.240 \\
\hline 18 & 0.41 & 0.02 & 0.39 & 0.44 & 0.098 \\
\hline 19 & 0.41 & 0.01 & 0.39 & 0.42 & -0.740 \\
\hline 20 & 0.40 & 0.01 & 0.38 & 0.42 & 0.398 \\
\hline Avg. & $\mathbf{0 . 4 1}$ & $\mathbf{0 . 0 1}$ & $\mathbf{0 . 3 9}$ & $\mathbf{0 . 4 3}$ & $\mathbf{0 . 1 0 8}$ \\
\hline Exp. & $\mathbf{0 . 4 4}$ & $\mathbf{0 . 0 3}$ & $\mathbf{0 . 4 1}$ & $\mathbf{0 . 4 4}$ & $\mathbf{- 0 . 0 2 2}$ \\
\hline \hline & & & & & \\
\hline
\end{tabular}


Table F.8 - LVL Compression $E_{z}(\mathrm{GPa})$

\begin{tabular}{lrrrrr}
\hline Sim. & Mean & STD & Min. & Max & Skew. \\
\hline 1 & 0.80 & 0.04 & 0.74 & 0.86 & -0.019 \\
2 & 0.83 & 0.04 & 0.79 & 0.90 & 0.499 \\
3 & 0.81 & 0.04 & 0.76 & 0.87 & 0.606 \\
4 & 0.84 & 0.05 & 0.75 & 0.89 & -0.313 \\
\hline 5 & 0.81 & 0.03 & 0.78 & 0.86 & 0.340 \\
6 & 0.81 & 0.04 & 0.77 & 0.90 & 1.078 \\
7 & 0.82 & 0.02 & 0.80 & 0.86 & 0.698 \\
8 & 0.83 & 0.02 & 0.79 & 0.85 & -0.098 \\
9 & 0.82 & 0.04 & 0.75 & 0.89 & 0.167 \\
10 & 0.82 & 0.04 & 0.75 & 0.88 & -0.187 \\
11 & 0.82 & 0.02 & 0.77 & 0.85 & -0.469 \\
12 & 0.81 & 0.04 & 0.75 & 0.87 & -0.025 \\
13 & 0.82 & 0.02 & 0.78 & 0.85 & -0.304 \\
14 & 0.81 & 0.04 & 0.76 & 0.89 & 0.554 \\
15 & 0.82 & 0.02 & 0.77 & 0.85 & -0.691 \\
16 & 0.81 & 0.03 & 0.75 & 0.88 & 0.309 \\
\hline 17 & 0.82 & 0.03 & 0.78 & 0.87 & -0.152 \\
18 & 0.81 & 0.04 & 0.76 & 0.87 & 0.269 \\
\hline 19 & 0.82 & 0.03 & 0.76 & 0.87 & 0.045 \\
20 & 0.81 & 0.04 & 0.77 & 0.88 & 0.609 \\
\hline Avg. & $\mathbf{0 . 8 2}$ & $\mathbf{0 . 0 3}$ & $\mathbf{0 . 7 7}$ & $\mathbf{0 . 8 7}$ & $\mathbf{0 . 1 4 6}$ \\
\hline Exp. & $\mathbf{0 . 3 7}$ & $\mathbf{0 . 0 3}$ & $\mathbf{0 . 3 1}$ & $\mathbf{0 . 4 2}$ & $\mathbf{0 . 1 3 5}$ \\
\hline \hline & & & & & \\
\hline
\end{tabular}

Table F.9 - LVL Compression $E_{45^{\circ}}^{0^{\circ}}(\mathrm{GPa})$

\begin{tabular}{lrrrrr}
\hline \hline Sim. & Mean & STD & Min. & Max & Skew. \\
\hline 1 & 0.79 & 0.03 & 0.76 & 0.84 & 0.267 \\
2 & 0.80 & 0.02 & 0.77 & 0.82 & -0.262 \\
\hline 3 & 0.79 & 0.02 & 0.74 & 0.82 & -0.567 \\
4 & 0.80 & 0.03 & 0.77 & 0.84 & 0.229 \\
\hline 5 & 0.79 & 0.03 & 0.77 & 0.85 & 0.968 \\
6 & 0.78 & 0.02 & 0.76 & 0.83 & 0.748 \\
\hline 7 & 0.78 & 0.02 & 0.75 & 0.80 & -0.253 \\
8 & 0.80 & 0.04 & 0.75 & 0.87 & 0.504 \\
\hline 9 & 0.81 & 0.02 & 0.77 & 0.86 & 0.139 \\
10 & 0.79 & 0.03 & 0.74 & 0.84 & 0.097 \\
\hline 11 & 0.80 & 0.02 & 0.77 & 0.83 & 0.026 \\
12 & 0.79 & 0.02 & 0.75 & 0.83 & 0.612 \\
13 & 0.77 & 0.02 & 0.74 & 0.81 & 0.023 \\
14 & 0.78 & 0.03 & 0.75 & 0.82 & -0.056 \\
\hline 15 & 0.78 & 0.03 & 0.74 & 0.83 & 0.449 \\
\hline 16 & 0.78 & 0.02 & 0.74 & 0.80 & -0.726 \\
\hline 17 & 0.79 & 0.03 & 0.76 & 0.84 & 0.271 \\
\hline 18 & 0.79 & 0.03 & 0.74 & 0.84 & 0.049 \\
\hline 19 & 0.79 & 0.02 & 0.76 & 0.81 & -0.699 \\
\hline 20 & 0.77 & 0.03 & 0.74 & 0.81 & 0.367 \\
\hline Avg. & $\mathbf{0 . 7 9}$ & $\mathbf{0 . 0 2}$ & $\mathbf{0 . 7 5}$ & $\mathbf{0 . 8 3}$ & $\mathbf{0 . 1 0 9}$ \\
\hline Exp. & $\mathbf{0 . 8 7}$ & $\mathbf{0 . 0 7}$ & $\mathbf{0 . 7 6}$ & $\mathbf{1 . 0 1}$ & $\mathbf{0 . 0 0 4}$ \\
\hline \hline & & & & & \\
\hline
\end{tabular}


Table F.10 - LVL Compression $E_{45^{\circ}}^{90^{\circ}}(\mathrm{GPa})$

\begin{tabular}{lrrrrr}
\hline Sim. & Mean & STD & Min. & Max & Skew. \\
\hline 1 & 1.49 & 0.06 & 1.39 & 1.60 & 0.019 \\
2 & 1.55 & 0.07 & 1.47 & 1.66 & 0.485 \\
3 & 1.50 & 0.06 & 1.42 & 1.61 & 0.591 \\
4 & 1.55 & 0.08 & 1.40 & 1.65 & -0.333 \\
\hline 5 & 1.51 & 0.05 & 1.45 & 1.58 & 0.274 \\
6 & 1.52 & 0.06 & 1.44 & 1.66 & 1.029 \\
7 & 1.53 & 0.04 & 1.49 & 1.60 & 0.555 \\
8 & 1.54 & 0.04 & 1.48 & 1.59 & -0.053 \\
9 & 1.53 & 0.06 & 1.40 & 1.65 & -0.034 \\
10 & 1.53 & 0.08 & 1.40 & 1.63 & -0.190 \\
11 & 1.52 & 0.04 & 1.45 & 1.57 & -0.408 \\
12 & 1.51 & 0.07 & 1.39 & 1.63 & 0.004 \\
13 & 1.52 & 0.04 & 1.46 & 1.57 & -0.331 \\
14 & 1.51 & 0.07 & 1.41 & 1.64 & 0.486 \\
15 & 1.52 & 0.04 & 1.44 & 1.57 & -0.759 \\
16 & 1.51 & 0.06 & 1.41 & 1.64 & 0.290 \\
\hline 17 & 1.53 & 0.05 & 1.46 & 1.61 & -0.061 \\
18 & 1.50 & 0.07 & 1.42 & 1.61 & 0.316 \\
\hline 19 & 1.52 & 0.06 & 1.41 & 1.61 & 0.030 \\
\hline 20 & 1.51 & 0.07 & 1.44 & 1.62 & 0.570 \\
\hline Avg. & $\mathbf{1 . 5 2}$ & $\mathbf{0 . 0 6}$ & $\mathbf{1 . 4 3}$ & $\mathbf{1 . 6 1}$ & $\mathbf{0 . 1 2 4}$ \\
\hline Exp. & $\mathbf{0 . 8 4}$ & $\mathbf{0 . 0 6}$ & $\mathbf{0 . 7 5}$ & $\mathbf{0 . 9 6}$ & $\mathbf{0 . 5 4 5}$ \\
\hline \hline & & & & & \\
\hline
\end{tabular}

Table F.11 - LVL Compression $E_{90^{\circ}}^{45^{\circ}}(\mathrm{GPa})$

\begin{tabular}{lrrrrr}
\hline \hline Sim. & Mean & STD & Min. & Max & Skew. \\
\hline 1 & 0.48 & 0.02 & 0.46 & 0.51 & 0.078 \\
2 & 0.50 & 0.02 & 0.48 & 0.53 & 0.553 \\
3 & 0.49 & 0.02 & 0.47 & 0.51 & 0.587 \\
4 & 0.50 & 0.02 & 0.46 & 0.53 & -0.344 \\
\hline 5 & 0.49 & 0.01 & 0.47 & 0.50 & 0.112 \\
6 & 0.49 & 0.02 & 0.47 & 0.53 & 0.858 \\
7 & 0.49 & 0.01 & 0.48 & 0.50 & 0.460 \\
\hline 8 & 0.50 & 0.01 & 0.48 & 0.51 & -0.072 \\
9 & 0.50 & 0.01 & 0.48 & 0.53 & 1.207 \\
10 & 0.49 & 0.02 & 0.46 & 0.51 & -0.333 \\
11 & 0.49 & 0.01 & 0.47 & 0.51 & -0.410 \\
12 & 0.49 & 0.02 & 0.46 & 0.51 & -0.299 \\
13 & 0.49 & 0.01 & 0.47 & 0.50 & -0.631 \\
14 & 0.49 & 0.02 & 0.46 & 0.52 & 0.424 \\
15 & 0.49 & 0.01 & 0.47 & 0.50 & -0.198 \\
16 & 0.49 & 0.02 & 0.46 & 0.51 & 0.211 \\
\hline 17 & 0.49 & 0.01 & 0.47 & 0.51 & -0.060 \\
\hline 18 & 0.49 & 0.02 & 0.46 & 0.52 & 0.423 \\
\hline 19 & 0.49 & 0.01 & 0.47 & 0.52 & 0.253 \\
\hline 20 & 0.49 & 0.02 & 0.47 & 0.51 & 0.577 \\
\hline Avg. & $\mathbf{0 . 4 9}$ & $\mathbf{0 . 0 2}$ & $\mathbf{0 . 4 7}$ & $\mathbf{0 . 5 1}$ & $\mathbf{0 . 1 7 0}$ \\
\hline Exp. & $\mathbf{0 . 2 9}$ & $\mathbf{0 . 0 5}$ & $\mathbf{0 . 2 3}$ & $\mathbf{0 . 3 5}$ & $\mathbf{0 . 1 1 2}$ \\
\hline \hline
\end{tabular}


F.2 The properties of PSL

Table F.12 - PSL $\Sigma A_{i}\left(\mathrm{~cm}^{2}\right)$

\begin{tabular}{|c|c|c|c|c|c|}
\hline Sim. & Mean & STD & Min. & Max & Skewness \\
\hline 1 & 103.4 & 1.3 & 101.4 & 106.0 & 0.272 \\
\hline 2 & 103.8 & 1.3 & 101.7 & 106.2 & 0.024 \\
\hline 3 & 104.0 & 1.0 & 102.3 & 105.6 & -0.014 \\
\hline 4 & 103.2 & 1.5 & 101.3 & 105.8 & 0.303 \\
\hline 5 & 104.3 & 1.8 & 101.1 & 106.4 & -0.487 \\
\hline 6 & 103.9 & 1.6 & 101.2 & 106.3 & -0.034 \\
\hline 7 & 104.0 & 1.2 & 101.9 & 106.4 & 0.575 \\
\hline 8 & 103.8 & 1.5 & 101.1 & 106.4 & 0.127 \\
\hline 9 & 103.9 & 1.7 & 101.3 & 106.3 & -0.120 \\
\hline 10 & 103.6 & 1.2 & 101.4 & 106.3 & 0.203 \\
\hline 11 & 103.9 & 1.4 & 101.3 & 105.9 & -0.490 \\
\hline 12 & 103.8 & 1.3 & 101.2 & 105.8 & -0.386 \\
\hline 13 & 103.0 & 1.3 & 101.2 & 106.2 & 0.565 \\
\hline 14 & 103.8 & 1.5 & 101.5 & 106.1 & -0.067 \\
\hline 15 & 104.5 & 1.2 & 101.7 & 105.9 & -0.551 \\
\hline 16 & 103.7 & 1.2 & 101.2 & 105.9 & -0.104 \\
\hline 17 & 103.5 & 1.7 & 101.2 & 105.7 & 0.050 \\
\hline 18 & 103.2 & 1.6 & 101.2 & 106.1 & 0.247 \\
\hline 19 & 103.3 & 1.3 & 101.5 & 106.2 & 0.392 \\
\hline 20 & 103.7 & 1.8 & 101.1 & 106.3 & 0.071 \\
\hline Avg. & 103.7 & 1.4 & 101.4 & 106.1 & 0.029 \\
\hline \multicolumn{6}{|c|}{ Cross-section of the simulated beam: $106.5 \mathrm{~cm}^{2}$} \\
\hline
\end{tabular}


Table F.13 - PSL $\Sigma I_{i}\left(\mathrm{~cm}^{4}\right)-$ edgewise

\begin{tabular}{|c|c|c|c|c|c|}
\hline Sim. & Mean & STD & Min. & Max & Skew. \\
\hline 1 & 1682 & 22 & 1648 & 1724 & 0.254 \\
\hline 2 & 1688 & 22 & 1651 & 1726 & -0.055 \\
\hline 3 & 1691 & 16 & 1666 & 1718 & 0.128 \\
\hline 4 & 1678 & 24 & 1647 & 1720 & 0.340 \\
\hline 5 & 1697 & 29 & 1643 & 1730 & -0.521 \\
\hline 6 & 1690 & 26 & 1646 & 1728 & -0.021 \\
\hline 7 & 1691 & 19 & 1658 & 1730 & 0.576 \\
\hline 8 & 1688 & 24 & 1645 & 1729 & 0.131 \\
\hline 9 & 1691 & 28 & 1648 & 1730 & -0.095 \\
\hline 10 & 1686 & 20 & 1649 & 1729 & 0.175 \\
\hline 11 & 1690 & 22 & 1650 & 1723 & -0.392 \\
\hline 12 & 1689 & 20 & 1645 & 1721 & -0.387 \\
\hline 13 & 1675 & 21 & 1646 & 1727 & 0.612 \\
\hline 14 & 1689 & 24 & 1651 & 1728 & -0.062 \\
\hline 15 & 1699 & 21 & 1651 & 1725 & -0.528 \\
\hline 16 & 1686 & 19 & 1644 & 1722 & -0.228 \\
\hline 17 & 1683 & 27 & 1644 & 1720 & 0.003 \\
\hline 18 & 1679 & 26 & 1647 & 1725 & 0.245 \\
\hline 19 & 1680 & 23 & 1648 & 1727 & 0.306 \\
\hline 20 & 1687 & 29 & 1646 & 1730 & 0.090 \\
\hline Avg. & 1687 & 23 & 1649 & 1726 & 0.029 \\
\hline
\end{tabular}

APPENDIX F
Table F.14 - PSL $\Sigma I_{i}\left(\mathrm{~cm}^{4}\right)$ - flatwise

\begin{tabular}{|c|c|c|c|c|c|}
\hline Sim. & Mean & STD & Min. & Max & Skew. \\
\hline 1 & 497.0 & 6.0 & 487.6 & 509.9 & 0.321 \\
\hline 2 & 498.4 & 6.8 & 486.8 & 511.2 & 0.043 \\
\hline 3 & 499.9 & 4.8 & 490.5 & 507.1 & -0.277 \\
\hline 4 & 495.8 & 7.4 & 486.6 & 508.7 & 0.334 \\
\hline 5 & 501.5 & 8.8 & 485.5 & 512.7 & -0.523 \\
\hline 6 & 499.4 & 7.8 & 486.5 & 510.9 & -0.051 \\
\hline 7 & 499.6 & 5.7 & 490.6 & 510.8 & 0.576 \\
\hline 8 & 498.3 & 7.3 & 485.5 & 510.8 & 0.088 \\
\hline 9 & 499.3 & 8.3 & 486.0 & 511.1 & -0.126 \\
\hline 10 & 497.8 & 5.8 & 486.9 & 508.7 & -0.031 \\
\hline 11 & 499.3 & 6.7 & 486.2 & 509.3 & -0.478 \\
\hline 12 & 498.5 & 6.3 & 485.1 & 507.6 & -0.569 \\
\hline 13 & 495.0 & 6.1 & 485.7 & 509.7 & 0.542 \\
\hline 14 & 499.0 & 6.8 & 488.7 & 509.3 & -0.005 \\
\hline 15 & 501.4 & 6.2 & 487.2 & 508.7 & -0.564 \\
\hline 16 & 497.7 & 5.5 & 485.4 & 507.8 & -0.269 \\
\hline 17 & 496.8 & 7.9 & 486.0 & 508.4 & 0.097 \\
\hline 18 & 495.7 & 7.8 & 486.0 & 509.6 & 0.277 \\
\hline 19 & 496.7 & 6.2 & 488.4 & 510.6 & 0.476 \\
\hline 20 & 498.2 & 8.3 & 486.7 & 511.2 & 0.152 \\
\hline Avg. & 498.3 & 6.8 & 486.9 & 509.7 & 0.001 \\
\hline
\end{tabular}

Flatwise moment of inertia of the beam: $515.1 \mathrm{~cm}^{4}$ 
Table F.15 - PSL density $\left(\mathrm{kg} / \mathrm{m}^{3}\right)$

\begin{tabular}{lrrrrr}
\hline \hline Sim. & Mean & STD & Min. & Max & Skew. \\
\hline 1 & 674 & 11 & 656 & 704 & 0.500 \\
2 & 673 & 10 & 654 & 690 & -0.045 \\
3 & 671 & 10 & 648 & 686 & -0.504 \\
4 & 671 & 12 & 652 & 695 & 0.145 \\
\hline 5 & 679 & 15 & 658 & 705 & 0.021 \\
6 & 674 & 12 & 649 & 694 & -0.262 \\
7 & 673 & 9 & 661 & 689 & 0.227 \\
8 & 674 & 12 & 654 & 698 & 0.201 \\
9 & 675 & 13 & 652 & 701 & 0.267 \\
10 & 670 & 8 & 655 & 683 & -0.135 \\
11 & 674 & 13 & 647 & 699 & -0.190 \\
12 & 674 & 11 & 652 & 694 & -0.261 \\
13 & 669 & 12 & 645 & 694 & 0.200 \\
14 & 673 & 11 & 649 & 694 & -0.266 \\
15 & 675 & 10 & 660 & 697 & 0.400 \\
16 & 674 & 7 & 658 & 686 & -0.167 \\
\hline 17 & 674 & 12 & 643 & 691 & -0.705 \\
18 & 672 & 14 & 651 & 698 & 0.263 \\
\hline 19 & 673 & 11 & 652 & 690 & -0.285 \\
\hline 20 & 671 & 13 & 645 & 696 & -0.141 \\
\hline Avg. & $\mathbf{6 7 3}$ & $\mathbf{1 1}$ & $\mathbf{6 5 2}$ & $\mathbf{6 9 4}$ & $\mathbf{- 0 . 0 3 7}$ \\
\hline Exp. & $\mathbf{6 7 3}$ & $\mathbf{1 6}$ & $\mathbf{6 4 0}$ & $\mathbf{7 0 8}$ & $\mathbf{- 0 . 1 2 2}$ \\
\hline \hline
\end{tabular}

Table F.16 - PSL Bending MOE - edgewise

\begin{tabular}{lrrrrr}
\hline \hline Sim. & Mean & STD & Min. & Max & Skew. \\
\hline 1 & 14.38 & 0.35 & 13.82 & 15.35 & 0.654 \\
2 & 14.63 & 0.43 & 13.96 & 15.43 & 0.013 \\
\hline 3 & 14.49 & 0.42 & 13.84 & 15.32 & 0.222 \\
\hline 4 & 14.43 & 0.37 & 13.83 & 15.17 & 0.142 \\
\hline 5 & 14.53 & 0.45 & 13.71 & 15.22 & -0.087 \\
\hline 6 & 14.55 & 0.31 & 14.10 & 15.04 & 0.133 \\
\hline 7 & 14.69 & 0.37 & 14.00 & 15.37 & -0.025 \\
\hline 8 & 14.62 & 0.27 & 14.02 & 15.10 & 0.076 \\
\hline 9 & 14.63 & 0.39 & 13.45 & 15.11 & -1.289 \\
10 & 14.51 & 0.42 & 13.79 & 15.32 & 0.177 \\
\hline 11 & 14.56 & 0.30 & 14.04 & 15.17 & 0.036 \\
12 & 14.62 & 0.30 & 13.95 & 15.30 & 0.151 \\
\hline 13 & 14.50 & 0.40 & 13.68 & 15.43 & 0.060 \\
14 & 14.57 & 0.64 & 13.49 & 15.65 & 0.016 \\
\hline 15 & 14.51 & 0.46 & 13.48 & 15.11 & -0.852 \\
\hline 16 & 14.55 & 0.34 & 13.87 & 15.14 & -0.133 \\
\hline 17 & 14.65 & 0.40 & 13.74 & 15.37 & -0.290 \\
\hline 18 & 14.42 & 0.49 & 13.56 & 15.35 & 0.105 \\
\hline 19 & 14.46 & 0.44 & 13.73 & 15.43 & 0.251 \\
\hline 20 & 14.50 & 0.49 & 13.49 & 15.34 & -0.404 \\
\hline Avg. & $\mathbf{1 4 . 5 4}$ & $\mathbf{0 . 4 0}$ & $\mathbf{1 3 . 7 8}$ & $\mathbf{1 5 . 2 9}$ & $\mathbf{- 0 . 0 5 2}$ \\
\hline Exp. & $\mathbf{1 2 . 8 2}$ & $\mathbf{0 . 7 5}$ & $\mathbf{1 1 . 1 8}$ & $\mathbf{1 3 . 8 1}$ & $\mathbf{0 . 1 1 5}$ \\
\hline \hline & & & & & \\
\hline
\end{tabular}


Table F.17 - PSL Bending MOE - flatwise (GPa)

\begin{tabular}{lccccc}
\hline \hline Sim. & Mean & STD & Min. & Max & Skew. \\
\hline 1 & 14.39 & 0.28 & 13.99 & 14.97 & 0.471 \\
2 & 14.32 & 0.45 & 13.23 & 15.11 & -0.386 \\
3 & 14.38 & 0.37 & 13.77 & 15.22 & 0.389 \\
4 & 14.29 & 0.37 & 13.66 & 15.03 & 0.310 \\
\hline 5 & 14.53 & 0.32 & 14.05 & 15.13 & 0.102 \\
6 & 14.53 & 0.28 & 13.76 & 14.98 & -0.725 \\
7 & 14.57 & 0.41 & 13.75 & 15.27 & -0.298 \\
8 & 14.49 & 0.39 & 13.84 & 15.35 & 0.153 \\
9 & 14.63 & 0.24 & 14.07 & 15.07 & -0.432 \\
10 & 14.49 & 0.29 & 13.89 & 15.11 & 0.021 \\
11 & 14.47 & 0.34 & 13.78 & 15.07 & 0.092 \\
12 & 14.54 & 0.38 & 13.60 & 15.37 & -0.189 \\
13 & 14.33 & 0.30 & 13.74 & 15.09 & 0.565 \\
14 & 14.61 & 0.38 & 13.79 & 15.10 & -0.530 \\
15 & 14.51 & 0.28 & 13.87 & 15.00 & -0.122 \\
16 & 14.44 & 0.29 & 14.07 & 14.96 & 0.367 \\
\hline 17 & 14.53 & 0.30 & 14.10 & 15.11 & 0.324 \\
18 & 14.38 & 0.42 & 13.76 & 15.21 & 0.487 \\
\hline 19 & 14.48 & 0.37 & 13.93 & 15.10 & 0.158 \\
\hline 20 & 14.36 & 0.27 & 13.94 & 14.76 & 0.066 \\
\hline Avg. & $\mathbf{1 4 . 4 6}$ & $\mathbf{0 . 3 4}$ & $\mathbf{1 3 . 8 3}$ & $\mathbf{1 5 . 1 0}$ & $\mathbf{0 . 0 4 1}$ \\
\hline Exp. & $\mathbf{1 2 . 5 7}$ & $\mathbf{0 . 5 7}$ & $\mathbf{1 1 . 6 4}$ & $\mathbf{1 3 . 9 2}$ & $\mathbf{- 0 . 0 8 7}$ \\
\hline \hline & & & & & \\
\hline
\end{tabular}

Table F.18 - PSL Regression parameters

\begin{tabular}{lrll}
\hline \hline Sim. & \multicolumn{1}{c}{ b0 } & b1 & $\mathbf{r}^{\mathbf{2}}$ \\
\hline 1 & 2.654 & 0.815 & 0.413 \\
2 & 4.042 & 0.739 & 0.609 \\
3 & 11.991 & 0.174 & 0.024 \\
4 & 8.291 & 0.429 & 0.186 \\
5 & 0.270 & 0.982 & 0.504 \\
6 & 8.503 & 0.417 & 0.148 \\
7 & 6.586 & 0.556 & 0.395 \\
8 & 10.426 & 0.289 & 0.174 \\
9 & 3.927 & 0.732 & 0.199 \\
10 & 8.141 & 0.439 & 0.090 \\
11 & 7.666 & 0.477 & 0.285 \\
12 & 6.714 & 0.544 & 0.465 \\
13 & 2.693 & 0.824 & 0.368 \\
14 & -3.770 & 1.256 & 0.573 \\
\hline 15 & 2.105 & 0.855 & 0.280 \\
16 & 4.021 & 0.729 & 0.393 \\
\hline 17 & 4.517 & 0.697 & 0.279 \\
\hline 18 & 4.365 & 0.699 & 0.371 \\
\hline 19 & 0.087 & 0.993 & 0.728 \\
\hline 20 & -5.225 & 1.373 & 0.583 \\
\hline Avg. & $\mathbf{4 . 4 0 0}$ & $\mathbf{0 . 7 0 1}$ & $\mathbf{0 . 3 5 3}$ \\
\hline \hline
\end{tabular}


Table F.19 - PSL Compression $E_{x}(\mathrm{GPa})$

\begin{tabular}{lccccc}
\hline \hline Sim. & Mean & STD & Min. & Max & Skew. \\
\hline 1 & 13.50 & 0.47 & 12.53 & 14.05 & -0.868 \\
2 & 13.26 & 0.23 & 12.89 & 13.66 & 0.167 \\
3 & 13.31 & 0.63 & 12.30 & 14.35 & 0.183 \\
4 & 13.48 & 0.41 & 12.60 & 14.11 & -0.628 \\
\hline 5 & 13.43 & 0.33 & 12.91 & 13.93 & -0.327 \\
6 & 13.21 & 0.48 & 12.48 & 14.11 & 0.247 \\
7 & 13.34 & 0.26 & 12.85 & 13.63 & -0.590 \\
8 & 13.47 & 0.53 & 12.25 & 14.01 & -1.030 \\
9 & 13.37 & 0.48 & 12.51 & 14.35 & 0.239 \\
10 & 13.09 & 0.42 & 12.36 & 13.65 & -0.400 \\
11 & 13.35 & 0.55 & 12.53 & 14.01 & -0.269 \\
12 & 13.48 & 0.41 & 13.00 & 14.41 & 0.911 \\
13 & 13.25 & 0.51 & 12.62 & 13.89 & 0.021 \\
14 & 13.28 & 0.30 & 12.88 & 13.69 & -0.025 \\
15 & 12.99 & 0.46 & 12.33 & 13.94 & 0.606 \\
16 & 13.49 & 0.50 & 12.66 & 14.23 & -0.052 \\
17 & 13.28 & 0.36 & 12.48 & 13.60 & -0.938 \\
18 & 13.57 & 0.39 & 12.98 & 14.18 & -0.035 \\
\hline 19 & 13.24 & 0.50 & 12.38 & 13.85 & -0.246 \\
\hline 20 & 13.50 & 0.41 & 12.95 & 14.17 & 0.256 \\
\hline Avg. & $\mathbf{1 3 . 3 4}$ & $\mathbf{0 . 4 3}$ & $\mathbf{1 2 . 6 3}$ & $\mathbf{1 3 . 9 9}$ & $\mathbf{- 0 . 1 3 9}$ \\
\hline Exp. & $\mathbf{1 3 . 2 0}$ & $\mathbf{2 . 0 9}$ & $\mathbf{1 0 . 7 1}$ & $\mathbf{1 7 . 2 8}$ & $\mathbf{0 . 1 2 8}$ \\
\hline \hline & & & & & \\
\hline
\end{tabular}

Table F.20 - PSL Compression $E_{y}(\mathrm{GPa})$

\begin{tabular}{lrrrrr}
\hline \hline Sim. & Mean & STD & Min. & Max & Skew. \\
\hline 1 & 0.55 & 0.02 & 0.51 & 0.56 & -1.218 \\
2 & 0.54 & 0.02 & 0.52 & 0.58 & 0.456 \\
3 & 0.54 & 0.03 & 0.50 & 0.59 & 0.304 \\
4 & 0.54 & 0.02 & 0.52 & 0.56 & -0.055 \\
\hline 5 & 0.54 & 0.01 & 0.52 & 0.56 & 0.211 \\
6 & 0.54 & 0.01 & 0.53 & 0.58 & 1.089 \\
\hline 7 & 0.54 & 0.01 & 0.52 & 0.55 & -0.941 \\
\hline 8 & 0.54 & 0.02 & 0.51 & 0.56 & -0.215 \\
9 & 0.54 & 0.01 & 0.51 & 0.57 & -0.251 \\
10 & 0.53 & 0.01 & 0.51 & 0.54 & 0.065 \\
11 & 0.54 & 0.01 & 0.51 & 0.56 & -0.398 \\
12 & 0.54 & 0.01 & 0.53 & 0.55 & 0.239 \\
13 & 0.54 & 0.01 & 0.53 & 0.55 & 0.313 \\
14 & 0.54 & 0.02 & 0.51 & 0.56 & -0.514 \\
15 & 0.54 & 0.01 & 0.51 & 0.56 & -0.674 \\
16 & 0.54 & 0.02 & 0.51 & 0.56 & -0.280 \\
\hline 17 & 0.54 & 0.02 & 0.52 & 0.56 & -0.271 \\
\hline 18 & 0.54 & 0.01 & 0.53 & 0.56 & 0.387 \\
\hline 19 & 0.53 & 0.01 & 0.51 & 0.55 & -1.580 \\
\hline 20 & 0.54 & 0.02 & 0.52 & 0.58 & 0.516 \\
\hline Avg. & $\mathbf{0 . 5 4}$ & $\mathbf{0 . 0 1}$ & $\mathbf{0 . 5 2}$ & $\mathbf{0 . 5 6}$ & $\mathbf{- 0 . 1 4 1}$ \\
\hline Exp. & $\mathbf{0 . 4 8}$ & $\mathbf{0 . 0 7}$ & $\mathbf{0 . 3 9}$ & $\mathbf{0 . 6 0}$ & $\mathbf{- 0 . 2 2 1}$ \\
\hline \hline & & & & & \\
\hline
\end{tabular}


Table F.21 - PSL Compression $E_{z}(\mathrm{GPa})$

\begin{tabular}{lrrrrr}
\hline Sim. & Mean & STD & Min. & Max & Skew. \\
\hline 1 & 1.03 & 0.03 & 0.98 & 1.06 & -0.802 \\
2 & 1.02 & 0.04 & 0.96 & 1.09 & 0.378 \\
3 & 1.03 & 0.06 & 0.97 & 1.15 & 0.684 \\
4 & 1.02 & 0.03 & 0.97 & 1.07 & 0.205 \\
\hline 5 & 1.02 & 0.04 & 0.96 & 1.06 & -0.349 \\
6 & 1.04 & 0.03 & 0.99 & 1.10 & 0.171 \\
7 & 1.01 & 0.03 & 0.95 & 1.07 & -0.079 \\
8 & 1.02 & 0.02 & 0.97 & 1.05 & -0.743 \\
9 & 1.04 & 0.02 & 1.00 & 1.07 & -0.128 \\
10 & 1.01 & 0.04 & 0.95 & 1.05 & -0.237 \\
\hline 11 & 1.02 & 0.03 & 0.96 & 1.06 & -0.801 \\
12 & 1.01 & 0.03 & 0.97 & 1.07 & 0.293 \\
13 & 1.03 & 0.03 & 0.99 & 1.08 & 0.361 \\
14 & 1.05 & 0.04 & 0.99 & 1.11 & 0.119 \\
15 & 1.01 & 0.03 & 0.93 & 1.04 & -1.180 \\
16 & 1.02 & 0.05 & 0.96 & 1.11 & 0.498 \\
\hline 17 & 1.03 & 0.04 & 0.98 & 1.10 & 0.715 \\
\hline 18 & 1.02 & 0.04 & 0.94 & 1.09 & -0.216 \\
\hline 19 & 1.04 & 0.02 & 1.00 & 1.07 & -0.202 \\
\hline 20 & 1.03 & 0.05 & 0.96 & 1.11 & 0.254 \\
\hline Avg. & $\mathbf{1 . 0 2}$ & $\mathbf{0 . 0 4}$ & $\mathbf{0 . 9 7}$ & $\mathbf{1 . 0 8}$ & $\mathbf{- 0 . 0 5 3}$ \\
\hline Exp. & $\mathbf{0 . 2 3}$ & $\mathbf{0 . 0 3}$ & $\mathbf{0 . 1 9}$ & $\mathbf{0 . 2 8}$ & $\mathbf{- 0 . 2 5 4}$ \\
\hline \hline
\end{tabular}

Table F.22 - PSL Compression $E_{45^{\circ}}^{0^{\circ}}(\mathrm{GPa})$

\begin{tabular}{lrrrrr}
\hline Sim. & Mean & STD & Min. & Max & Skew. \\
\hline 1 & 1.07 & 0.05 & 0.96 & 1.14 & -0.679 \\
2 & 1.07 & 0.04 & 1.02 & 1.13 & 0.263 \\
3 & 1.05 & 0.05 & 0.98 & 1.15 & 0.323 \\
4 & 1.06 & 0.03 & 1.01 & 1.10 & -0.032 \\
\hline 5 & 1.06 & 0.02 & 1.02 & 1.09 & 0.007 \\
6 & 1.06 & 0.02 & 1.02 & 1.10 & 0.187 \\
\hline 7 & 1.06 & 0.03 & 1.00 & 1.10 & -0.550 \\
8 & 1.07 & 0.04 & 1.01 & 1.13 & 0.096 \\
\hline 9 & 1.05 & 0.04 & 0.98 & 1.12 & 0.200 \\
10 & 1.03 & 0.03 & 0.98 & 1.07 & -0.500 \\
11 & 1.06 & 0.04 & 0.99 & 1.13 & -0.045 \\
12 & 1.06 & 0.03 & 1.03 & 1.13 & 0.659 \\
\hline 13 & 1.05 & 0.02 & 1.03 & 1.07 & -0.484 \\
14 & 1.05 & 0.04 & 1.00 & 1.10 & -0.446 \\
\hline 15 & 1.06 & 0.03 & 1.01 & 1.11 & 0.067 \\
16 & 1.05 & 0.05 & 0.98 & 1.12 & -0.053 \\
\hline 17 & 1.07 & 0.04 & 0.99 & 1.13 & -0.448 \\
\hline 18 & 1.05 & 0.04 & 1.00 & 1.11 & -0.060 \\
\hline 19 & 1.04 & 0.03 & 0.99 & 1.08 & -0.268 \\
\hline 20 & 1.08 & 0.03 & 1.03 & 1.13 & 0.144 \\
\hline Avg. & $\mathbf{1 . 0 6}$ & $\mathbf{0 . 0 4}$ & $\mathbf{1 . 0 0}$ & $\mathbf{1 . 1 1}$ & $\mathbf{- 0 . 0 8 1}$ \\
\hline Exp. & $\mathbf{1 . 0 8}$ & $\mathbf{0 . 1 1}$ & $\mathbf{0 . 8 6}$ & $\mathbf{1 . 2 5}$ & $\mathbf{- 0 . 0 0 2}$ \\
\hline \hline & & & & & \\
\hline
\end{tabular}


Table F.23 - PSL Compression $E_{45^{\circ}}^{90^{\circ}}(\mathrm{GPa})$

\begin{tabular}{lrrrrr}
\hline Sim. & Mean & STD & Min. & Max & Skew. \\
\hline 1 & 1.90 & 0.06 & 1.81 & 1.98 & -0.536 \\
2 & 1.89 & 0.07 & 1.78 & 2.00 & 0.245 \\
3 & 1.90 & 0.10 & 1.78 & 2.12 & 0.629 \\
4 & 1.88 & 0.04 & 1.82 & 1.94 & 0.189 \\
\hline 5 & 1.89 & 0.06 & 1.80 & 1.95 & -0.424 \\
6 & 1.91 & 0.05 & 1.85 & 2.00 & 0.164 \\
7 & 1.88 & 0.06 & 1.77 & 1.98 & -0.103 \\
8 & 1.90 & 0.05 & 1.80 & 1.97 & -0.292 \\
9 & 1.92 & 0.04 & 1.86 & 1.97 & 0.085 \\
10 & 1.86 & 0.07 & 1.77 & 1.96 & -0.094 \\
11 & 1.89 & 0.06 & 1.78 & 1.96 & -0.649 \\
12 & 1.87 & 0.04 & 1.81 & 1.95 & 0.205 \\
13 & 1.90 & 0.05 & 1.83 & 1.98 & -0.072 \\
14 & 1.93 & 0.07 & 1.84 & 2.02 & -0.012 \\
15 & 1.86 & 0.05 & 1.73 & 1.91 & -1.191 \\
16 & 1.89 & 0.09 & 1.78 & 2.05 & 0.480 \\
\hline 17 & 1.90 & 0.07 & 1.82 & 2.04 & 0.846 \\
18 & 1.89 & 0.08 & 1.75 & 2.04 & -0.004 \\
\hline 19 & 1.91 & 0.04 & 1.84 & 1.96 & -0.412 \\
\hline 20 & 1.92 & 0.08 & 1.81 & 2.05 & 0.237 \\
\hline Avg. & $\mathbf{1 . 9 0}$ & $\mathbf{0 . 0 6}$ & $\mathbf{1 . 8 0}$ & $\mathbf{1 . 9 9}$ & $\mathbf{- 0 . 0 3 5}$ \\
\hline Exp. & $\mathbf{0 . 6 4}$ & $\mathbf{0 . 0 5}$ & $\mathbf{0 . 5 5}$ & $\mathbf{0 . 7 3}$ & $\mathbf{- 0 . 1 0 0}$ \\
\hline \hline & & & & & \\
\hline
\end{tabular}

Table F.24 - PSL Compression $E_{90^{\circ}}^{45^{\circ}}(\mathrm{GPa})$

\begin{tabular}{lrrrrr}
\hline Sim. & Mean & STD & Min. & Max & Skew. \\
\hline 1 & 0.64 & 0.02 & 0.61 & 0.66 & -0.922 \\
2 & 0.64 & 0.03 & 0.61 & 0.68 & 0.219 \\
3 & 0.64 & 0.03 & 0.60 & 0.71 & 0.799 \\
4 & 0.64 & 0.01 & 0.61 & 0.66 & -0.054 \\
\hline 5 & 0.64 & 0.02 & 0.61 & 0.66 & -0.214 \\
6 & 0.65 & 0.02 & 0.62 & 0.68 & 0.368 \\
7 & 0.63 & 0.01 & 0.61 & 0.66 & 0.042 \\
8 & 0.64 & 0.01 & 0.61 & 0.66 & -0.456 \\
9 & 0.64 & 0.01 & 0.63 & 0.67 & 0.133 \\
10 & 0.63 & 0.02 & 0.61 & 0.65 & 0.281 \\
11 & 0.64 & 0.01 & 0.61 & 0.66 & -0.459 \\
12 & 0.63 & 0.02 & 0.61 & 0.66 & 0.143 \\
13 & 0.64 & 0.02 & 0.61 & 0.67 & -0.048 \\
14 & 0.65 & 0.02 & 0.62 & 0.67 & -0.135 \\
\hline 15 & 0.63 & 0.02 & 0.60 & 0.64 & -1.028 \\
\hline 16 & 0.64 & 0.03 & 0.60 & 0.68 & 0.343 \\
\hline 17 & 0.64 & 0.02 & 0.62 & 0.69 & 0.569 \\
\hline 18 & 0.64 & 0.02 & 0.60 & 0.68 & 0.070 \\
\hline 19 & 0.64 & 0.02 & 0.61 & 0.66 & -0.758 \\
\hline 20 & 0.65 & 0.02 & 0.61 & 0.69 & 0.407 \\
\hline Avg. & $\mathbf{0 . 6 4}$ & $\mathbf{0 . 0 2}$ & $\mathbf{0 . 6 1}$ & $\mathbf{0 . 6 7}$ & $\mathbf{- 0 . 0 3 5}$ \\
\hline Exp. & $\mathbf{0 . 3 0}$ & $\mathbf{0 . 0 3}$ & $\mathbf{0 . 2 5}$ & $\mathbf{0 . 3 6}$ & $\mathbf{0 . 1 8 6}$ \\
\hline \hline & & & & & \\
\hline
\end{tabular}




\section{VITA}

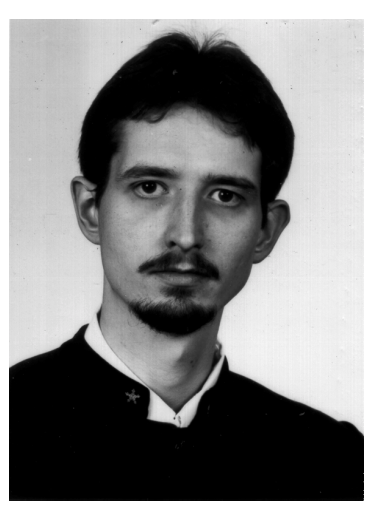

The author was born in Budapest, Hungary on August 30, 1973. He graduated from Kozma Lajos Technical High School in Budapest, Hungary, in 1992.

The author received his M.S. in Forest Products Technology at Brunnel University, England, and his M.S. in Wood Science and Technology from the University of Sopron, Hungary, in March and June 1998, respectively. He enrolled in West Virginia University for a Ph.D. degree in Forest Resource Science in August 1998, and completed this degree in August 2001. 